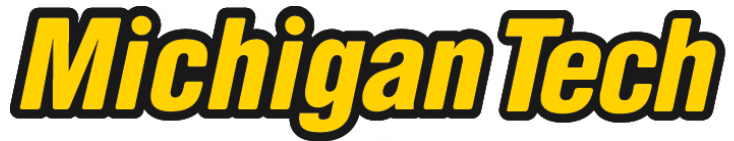 \\ Michigan Technological University Create the Future Digital Commons @ Michigan Tech
}

\section{Hall-effect thruster-cathode coupling : the effect of cathode position and magnetic field topology}

Jason D. Sommerville

Michigan Technological University

Follow this and additional works at: https://digitalcommons.mtu.edu/etds

Part of the Mechanical Engineering Commons

Copyright 2009 Jason D. Sommerville

\section{Recommended Citation}

Sommerville, Jason D., "Hall-effect thruster-cathode coupling : the effect of cathode position and magnetic field topology", Dissertation, Michigan Technological University, 2009.

https://doi.org/10.37099/mtu.dc.etds/411

Follow this and additional works at: https://digitalcommons.mtu.edu/etds

Part of the Mechanical Engineering Commons 


\title{
Hall-Effect Thruster-Cathode Coupling The Effect of Cathode Position and Magnetic Field Topology
}

\author{
by \\ Jason D. Sommerville \\ A Dissertation \\ Submitted in partial fulfillment of the requirements for the degree of \\ Doctor of Philosophy in Mechanical Engineering - Engineering Mechanics
}

Michigan TECHNOLOGICAL UNIVERSITY

2009 
Copyright (C) 2009 Jason D. Sommerville 


\section{Michigan TeChNOLOGICAL University Department of Mechanical Engineering - Engineering Mechanics}

This dissertation, entitled Hall-Effect Thruster-Cathode Coupling: The Effect of Cathode Position and Magnetic Field Topology, by Jason D. Sommerville is hereby accepted in partial fulfillment of the requirements for the degree of Doctor of Philosophy in Mechanical Engineering - Engineering Mechanics.

Signed:

Dissertation Advisor:

Dr. Lyon B. King

Date

Department Chair:

Dr. William W. Predebon

Date 

For my children, Tobias, Zachary, and Eliana. If, one day, you are thumbing though our bookshelves and come across this volume, don't feel obligated to read it. Hopefully the fact that you're looking for something to read means that I've instilled a love of learning in you. 



\begin{abstract}
Hall-effect thruster (HET) cathodes are responsible for the generation of the free electrons necessary to initiate and sustain the main plasma discharge and to neutralize the ion beam. The position of the cathode relative to the thruster strongly affects the efficiency of thrust generation. However, the mechanisms by which the position affects the efficiency are not well understood. This dissertation explores the effect of cathode position on HET efficiency. Magnetic field topology is shown to play an important role in the coupling between the cathode plasma and the main discharge plasma. The position of the cathode within the magnetic field affects the ion beam and the plasma properties of the near-field plume, which explains the changes in efficiency of the thruster.

Several experiments were conducted which explored the changes of efficiency arising from changes in cathode coupling. In each experiment, the thrust, discharge current, and cathode coupling voltage were monitored while changes in the independent variables of cathode position, cathode mass flow and magnetic field topology were made. From the telemetry data, the efficiency of the HET thrust generation was calculated. Furthermore, several ion beam and plasma properties were measured including ion energy distribution, beam current density profile, near-field plasma potential, electron temperature, and electron density. The ion beam data show how the independent variables affected the quality of ion beam and therefore the efficiency of thrust generation. The measurements of near-field plasma properties partially explain how the changes in ion beam quality arise.

The results of the experiments show that cathode position, mass flow, and field topology affect several aspects of the HET operation, especially beam divergence and voltage utilization efficiencies. Furthermore, the experiments show that magnetic field topology is important in the cathode coupling process. In particular, the magnetic field separatrix plays a critical role in impeding the coupling between cathode and HET. Suggested changes to HET thruster designs are provided including ways to improve the position of the separatrix to accommodate the cathode.
\end{abstract}




\section{Acknowledgments}

No graduate student succeeds alone, and I am no exception. There are numerous people to be acknowledged. First, my adviser, Dr. Lyon B. King, has been committed to my success. Having already seen me through my master's thesis, he has continued to support me throughout my doctoral work. His insights are sharp and have helped to clarify my thinking and methods. On the rare occasions that I have taken a question to him and he hasn't known the answer, the ensuing conversations were always encouraging. To a graduate student, whose time often seems like a self-chosen purgatory, this encouragement is vital.

I thank my committee, Dr. Gordon Parker, Dr. George Williams (NASA-Glenn) and Dr. Rainer A. Dresser (Spectral Sciences, Inc.), for the time they spent reading, correcting, and discussing this document with me. Committee members receive no remuneration for their services, save my thanks and a copy of this dissertation. A willingness to tackle a 300-page document gratis shows a great deal of support for me, and I am grateful.

The members of the ISP Laboratory, both past and present, have also been a great support. First off, Dr. Alex Kieckhafer and Dr. Dean Massey both took their time to show me the ropes of operations in the lab. Alex also provided numerous lunches full of delightful conversation. Emily Fossum has provided me her expertise in collisions and HET dynamics upon the asking. Jerry Ross has been a constant friend, and an accommodating person with whom to share a vaccum chamber. The graduate students who started after me, Jason Makela, Carrie Niemela, and Rob Washaleski have been kind enough to frequently ask me questions to which I knew the answers. I presume they did this primarily to boost by ego, though my wife would argue that that is hardly necessary. Finally, I thank the ISP undergraduates, Aaron Wendzel and Mark Hopkins, for their assistance in building and operating parts of my experiments.

Machining was provided for the experiments discussed in this work by Marty Toth. His expertise and willingness to work with me to turn napkin sketches into the necessary stands, mounts, and other pieces and parts were appreciated.

I'd like to thank Scott Austin and the people of Saints Peter and Paul Lutheran Church for providing spiritual support and guidance throughout the process of writing this dissertation. My growth in faith during this period of my life has been in equal measure to my growth in science.

Finally, and most importantly, I'd like to thank my family as a whole, and my dear wife, Renata, in particular, for providing steadfast support through these years. My work as a doctoral student has been no small sacrifice for her, as I have dragged her to the end of the Earth and asked her to live below the poverty level for some time. Despite this, our time here has also yielded Tobias, Zachary and Eliana, and their blessing has cheered me greatly during this time.

Funding for the research came from the Air Force Office of Scientific Research. 


\section{About the Electronic Edition}

This is the electronic edition of this document. There are a few differences between it and the print edition. Most noteably, there is an animation in Appendix B. This animation is known to work in version 8 of Adobe Acrobat Reader. It may not display correctly in other PDF viewers.

In addition, there are other subtle differences between this version and the print edition. Most the table of contents, list of figures, list of tables, citations, and crossreferences are hyperlinked. Hyperlink text appears in dark blue. Because of these hyperlinks, citation listings are not compressed. That is, a citation series will appear as "some fact ${ }^{1,2,3}$ " rather than "some fact ${ }^{1-3}$ ".

It has been noted that, depending on the PDF viewer, the magnetic field lines in some figures obscure the underlying data (c.f. Figure 6.8). This problem does not occur when viewing the document with Acrobat Reader. If you, the reader, are having this difficulty, the author suggests that you either try another PDF viewer, or simply print the figure in question.

The author considers this edition to be the canonical edition of the document. On the other hand, the print edition makes for a better coffee-table book. 


\section{Contents}

$\begin{array}{lll}\text { Abstract } & \text { vii }\end{array}$

$\begin{array}{ll}\text { Acknowlegements } & \text { ix }\end{array}$

About the Electronic Edition $\quad$ xi

$\begin{array}{ll}\text { List of Figures } & \text { xvii }\end{array}$

$\begin{array}{ll}\text { List of Tables } & \text { xxiii }\end{array}$

Nomenclature $\quad \mathbf{x x v}$

1 Introduction 1

1.1 Hall-Effect Thruster-Cathode Coupling and Performance . . . . . . . . 1

1.2 Contribution of this Work . . . . . . . . . . . . . . . 3

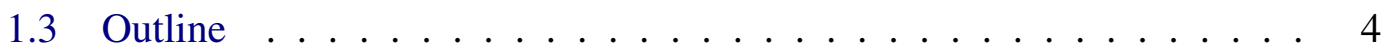

References for Chapter $1 \ldots \ldots \ldots 4$

2 Background $\quad 7$

2.1 Electron Motion in Magnetized Plasmas . . . . . . . . . . . . . . 7

2.1.1 Charged particle motion in a uniform magnetic field . . . . . 8

2.1.2 Charged particle motion in crossed electric and magnetic fields . 9

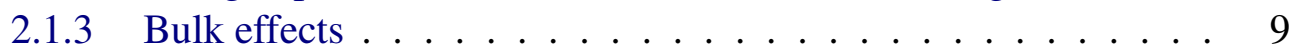

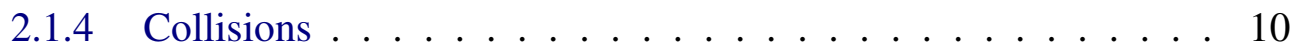

2.1.5 Electron diffusion and mobility . . . . . . . . . . 11

2.2 Hall-Effect Thrusters . . . . . . . . . . . . . . . . . . 13

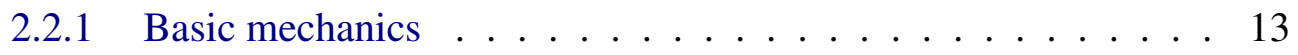

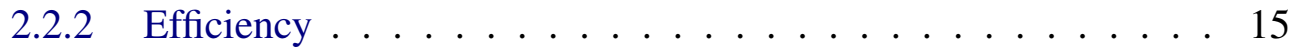

2.2.3 Efficiency loss mechanisms .................. 16

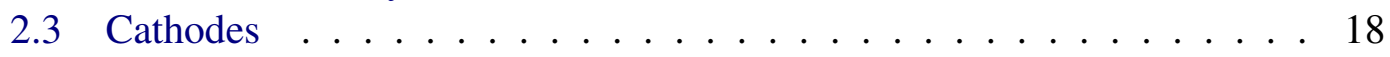

2.4 HET-Cathode Coupling . . . . . . . . . . . . . . 20 
References for Chapter $2 \ldots \ldots \ldots$. . . . . . . . . . . . 22

3 Review of Prior Research

3.1 Cathode Design . . . . . . . . . . . . . . . . 25

3.2 Early Work . . . . . . . . . . . . . . . . . 26

3.3 HET-Cathode Coupling . . . . . . . . . . . . . . . . 27

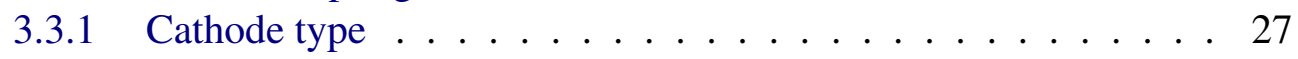

3.3.2 Cathode mass flow rate . . . . . . . . . . . . 27

3.3.3 Cathode position . . . . . . . . . . . . 28

3.4 Summary . . . . . . . . . . . . . . . . 30

References for Chapter $3 \ldots \ldots$. . . . . . . . . . . 30

4 Experiments $\quad 35$

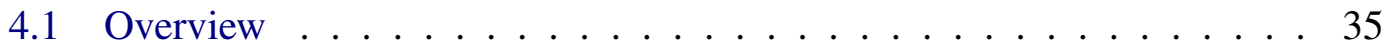

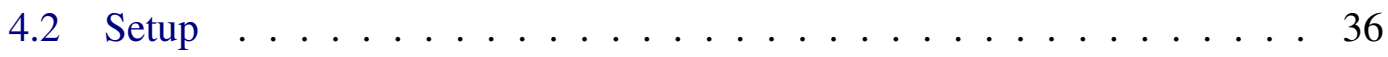

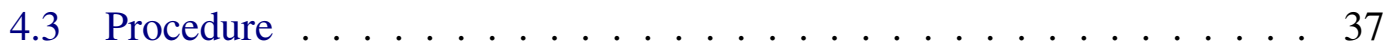

4.4 Equipment . . . . . . . . . . . . . . . . . . 39

4.4 .1 Diagnostics . . . . . . . . . . . . . . . . 39

4.4 .2 Vacuum facility ................... 40

4.4 .3 Hall thruster . . . . . . . . . . . . . . . . . . 40

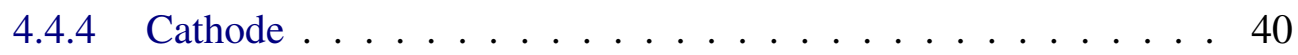

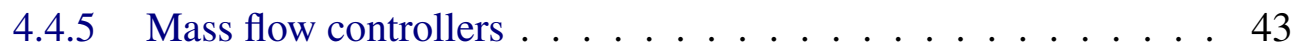

References for Chapter $4 \ldots \ldots$. . . . . . . . . . . . 43

5 Electrostatic Probes $\quad \mathbf{4 5}$

5.1 Langmuir Probe . . . . . . . . . . . . . . . . . . . . 45

5.2 Faraday Probe . . . . . . . . . . . . . . . . 48

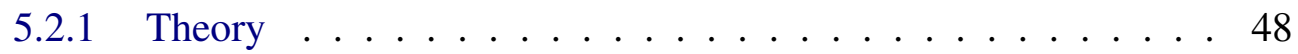

5.2 .2 Bias electronics . . . . . . . . . . . . . . . . . . 48

5.2 .3 Design and operation . . . . . . . . . . . . . 49

5.2 .4 Sources of error . . . . . . . . . . . . . . . . 51

5.3 Retarding Potential Analyzer . . . . . . . . . . . . . . . 52

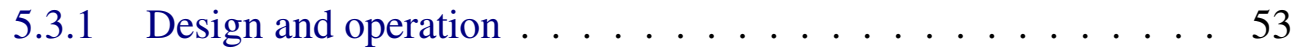

5.3 .2 RPA trace smoothing . . . . . . . . . . . . . 54

5.4 Double Probe . . . . . . . . . . . . . . . 55

5.4.1 Description and simple analysis ............ 55

5.4 .2 Peterson and Talbot analysis . . . . . . . . . . . 58

5.4 .3 Algorithm .............................. 60

5.4 Design and operation ................. 61

References for Chapter $5 \ldots \ldots$. . . . . . . . . . . 62 
6 Magnetic Field

6.1 Motivation . . . . . . . . . . . . . . . 65

6.2 Magnetic Field Model . . . . . . . . . . . . . . . . 66

6.2 .1 Model .................... 66

6.3 Model Results . . . . . . . . . . . . . . . . . . . . . . 67

6.4 Validation of the Model . . . . . . . . . . . . . . . . 68

6.5 Larmor Radius and Hall Parameter . . . . . . . . . . . . . . . . . 69

6.5.1 Larmor radius . . . . . . . . . . . . . . . . . . . . . . . 69

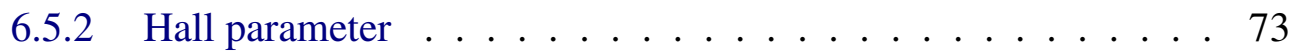

6.6 Discussion . . . . . . . . . . . . . . . . 75

6.7 Other HET Designs . . . . . . . . . . . . . . . . . . . 75

References for Chapter $6 \ldots \ldots \ldots$. . . . . . . . . . 78

7 Preliminary Experiments $\quad 79$

7.1 Cathode Position Experiment . . . . . . . . . . . . . . . . . . 79

7.2 Results . . . . . . . . . . . . . . . . . . 81

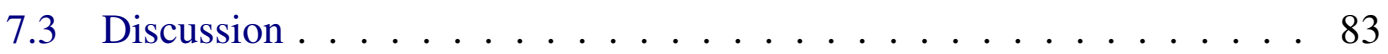

7.4 Performance vs. B Field . . . . . . . . . . . . . . . 86

References for Chapter $7 \ldots \ldots$. . . . . . . . . . . . 90

8 Separatrix Crossing Experiments $\quad 91$

8.1 Motivation and Overview . . . . . . . . . . . . . . . . . 91

8.2 Design of Experiment . . . . . . . . . . . . . . . . 93

8.2.1 Design of the extended outer pole . . . . . . . . . . 93

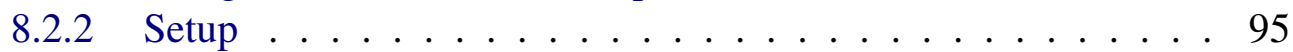

8.2 .3 Procedure ......................... 95

8.3 Performance ......................... 96

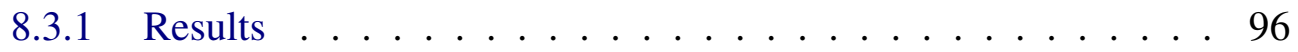

8.3 .2 Discussion ...................... 98

8.4 Ion Beam Properties . . . . . . . . . . . . . . . . . . . . . 99

8.4.1 Ion current density profiles . . . . . . . . . . . . . . 99

8.4.2 Ion energy distributions . . . . . . . . . . . . . . 100

8.5 Efficiency Analysis . . . . . . . . . . . . . . . . . 116

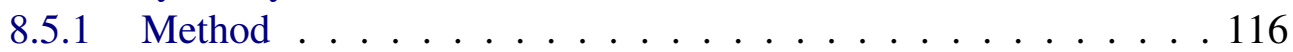

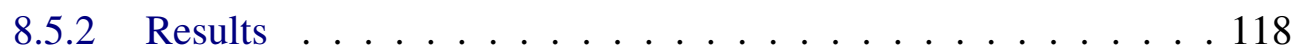

8.5 .3 Discussion ..................... . . 120

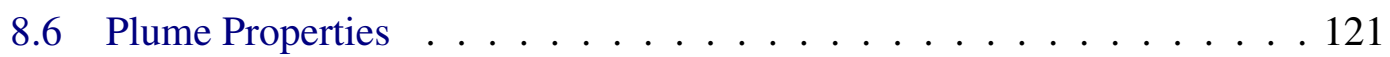

8.6.1 Results ..................... 121

8.6.2 Discussion ...................... . . 124

8.6.3 Average near-field plume properties . . . . . . . . . . . . 128

8.7 Discussion . . . . . . . . . . . . . . . . . . . . 134 
References for Chapter $8 \ldots \ldots \ldots$. . . . . . . . . . 140

9 Extended Outer Pole Performance 143

9.1 Motivation and Design of Experiment . . . . . . . . . . . . . . 143

9.2 Results . . . . . . . . . . . . . . . . . . . . . . 143

9.3 Discussion . . . . . . . . . . . . . . . . . . . . . . . 145

10 Conclusion 149

10.1 How Cathode Position Affects Efficiency _. . . . . . . . . . 149

10.2 How Cathode Position Affects Cathode Coupling . . . . . . . . . . . 151

10.3 The Separatrix . . . . . . . . . . . . . . . . . . . . 154

10.4 Future Work . . . . . . . . . . . . . . . . . . 155

10.5 Epilogue . . . . . . . . . . . . . . . . . 156

References for Chapter $10 \ldots \ldots \ldots \ldots$. . . . . . . . . . . . . . .

A Code Listings 157

A.1 Langmuir Probe Processing Algorithm . . . . . . . . . . . . . . 157

A.1.1 peterson.py . . . . . . . . . . . . . . . . 157

A.1.2 langmuir.py . . . . . . . . . . . . . . 163

A.2 Savitzky-Golay Smoothing Algorithm . . . . . . . . . . . . 172

A.3 Cai Plume Neutral Density Model . . . . . . . . . . . . . . . . . . 175

References for Appendix A . . . . . . . . . . . . . . . . . 177

B Double Probe Heating 179

C Plume Properties 183

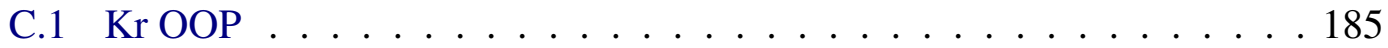

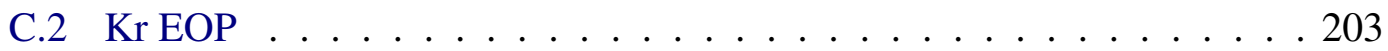

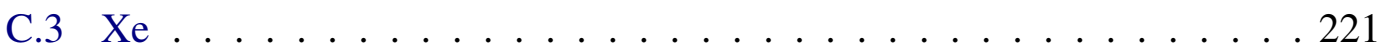

D Xe Separatrix Crossing Experiment 231

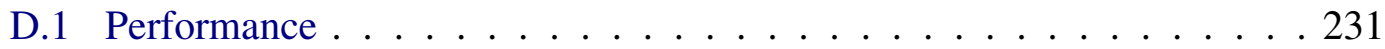

D.2 Faraday Probe Data . . . . . . . . . . . . . . . . 231

D.3 RPA Data . . . . . . . . . . . . . . . . . . . . . . . . . . 234

D.4 Efficiency Analysis . . . . . . . . . . . . . . . . . . . 234

References for Appendix D . . . . . . . . . . . . . . . . . . 239

E Single-Point Data $\quad 241$

F Permission to Use Material $\quad 245$ 


\section{List of Figures}

1.1 Photographs of an Aerojet BPT-2000 Hall-effect thruster . . . . . . . 2

1.2 The Busek BHT-200 Hall-effect thruster. (Photograph courtesy of Busek Co. Inc. See Appendix F) . . . . . . . . . . . . . . . . . . . 3

2.1 Motions of charged particles in (a) a constant magnetic field, and (b) crossed magnetic and electric fields . . . . . . . . . . 8

2.2 Electron-neutral momentum transfer and Coulomb collision cross sections and rate coefficients . . . . . . . . . . . . . . 11

2.3 (Left) Simplified schematic of Hall-effect thruster discharge chamber and associated processes (Right) An indication of the local plasma potential along the channel centerline . . . . . . . . . . . . 14

2.4 Efficiency loss mechanisms in a Hall-effect thruster . . . . . . . . 17

2.5 Cross-section of a typical orificed hollow cathode . . . . . . . . . 19

2.6 Coordinate system used to describe the cathode position . . . . . . 20

2.7 The effect of cathode coupling voltage on energy available for ion ac-

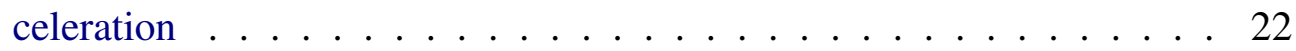

4.1 Experimental setup . . . . . . . . . . . . . . . 36

4.2 Photograph of experimental setup . . . . . . . . . . . 37

4.3 Electrical schematic of the HET and cathode . . . . . . . . . . . . 38

4.4 Schematic of the vacuum facility showing locations of key components 40

4.5 (a) Cut-away view of the laboratory cathode (b) Photograph (c) The heat shield (d) The heat shield blocks the beam, alleviating beam-induced cathode heating. . . . . . . . . . . . . . . . . 42

5.1 Typical and ideal Langmuir probe traces, showing the various regions of the trace . . . . . . . . . . . . . . . . 4 46

5.2 A sheath forms around Langmuir probes at high potentials . . . . . 46

5.3 Sheaths of a unguarded and guarded Faraday probe . . . . . . . . . 49

5.4 Schematic for the Faraday probe driver circuit . . . . . . . . . 50

5.5 Experimental measurement of gain for the Faraday probe driver circuit . 50

5.6 Schematic representation of an RPA . . . . . . . . . . . 53 
5.7 Sample RPA data, spline, and resulting IEDF . . . . . . . . . . . 55

5.8 A particularly noisy RPA trace showing the splines used to estimate the

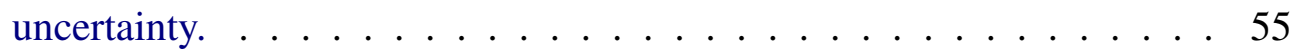

5.9 Typical double-Langmuir probe trace . . . . . . . . . . . . 56

5.10 Double probe sampling pattern . . . . . . . . . . . . . 62

6.1 Cross section of an HET showing the parts of the magnetic circuit . . . 66

6.2 Model of the simplified HET magnetic circuit. . . . . . . . . . . . 66

6.3 Magnetization of iron . . . . . . . . . . . . . . . . . 67

6.4 Model of the magnetic field structure of the BPT-2000 . . . . . . 70

6.5 Close-up of the anode region of the magnetic model . . . . . . . . 71

6.6 Magnetic field lines and separatrices for a typical HET. . . . . . . . . 72

6.7 Comparison of the modeled and measured magnetic fields with $4.2 \mathrm{~A}$ of magnet current . . . . . . . . . . . . . . . 72

6.8 Inputs and outputs used in estimating the magnetization of the plasma near the thruster . . . . . . . . . . . . . . . . . 73

6.9 Axisymmetric HET topology with different currents applied to the inner $\left(I_{\text {in }}\right)$, middle $\left(I_{\text {mid }}\right)$ and outer $\left(I_{\text {out }}\right)$ coils . . . . . . . . . 77

7.1 Experimental setup . . . . . . . . . . . . . . 80

7.2 Results of the cathode position test . . . . . . . . . . . . 84

7.3 Performance data overlaid with magnetic field lines. . . . . . . . . 88

8.1 Magnetic field lines and separatrices for a typical HET. . . . . . . . . . 92

8.2 Design and effect of the extended outer pole . . . . . . . . . . . . 94

8.3 Thruster performance as a function of cathode position while operating on krypton. . . . . . . . . . . . . . . . . . . . . 97

8.4 Ion beam current density as a function of off-axis angle while using the OOP. Each colored trace represents a different cathode location. . . . . 102

8.5 Ion beam current density as a function of off-axis angle while using the EOP. Each colored trace represents a different cathode location. . . . . 103

8.6 Ion energy distributions and ion current density as a function of angle for cathode mass flow rates of 2 SCCM with the OOP . . . . . . . . . 104

8.7 Ion energy distributions and ion current density as a function of angle for cathode mass flow rates of $5 \mathrm{SCCM}$ with the OOP . . . . . . . 106

8.8 Ion energy distributions and ion current density as a function of angle for cathode mass flow rates of $10 \mathrm{SCCM}$ with the OOP . . . . . . 108

8.9 Ion energy distributions and ion current density as a function of angle for cathode mass flow rates of $2 \mathrm{SCCM}$ with the EOP . . . . . . . 110

8.10 Ion energy distributions and ion current density as a function of angle for cathode mass flow rates of $5 \mathrm{SCCM}$ with the EOP . . . . . . . 112 
8.11 Ion energy distributions and ion current density as a function of angle for cathode mass flow rates of 10 SCCM with the EOP . . . . . . . 114

8.11 Ion energy distributions and ion current density as a function of angle for cathode mass flow rates of $10 \mathrm{SCCM}$ with the EOP (continued) . . 115

8.12 Efficiency component breakdown . . . . . . . . . . . . . . . . . . 119

8.13 Plasma properties on the thruster with the OOP with $\dot{m}_{c}=10 \mathrm{SCCM}$

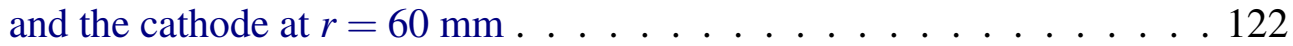

8.14 Plasma properties on the thruster with the OOP with $\dot{m}_{c}=10$ SCCM and the cathode at $r=120 \mathrm{~mm} \ldots \ldots \ldots 123$

8.15 Plasma potential plots for the thruster operating with the EOP and $\dot{m}_{c}=$ 10 SCCM. The near-field plasma potential increases with cathode postion. 126

8.16 Floating potential maps exhibit a double-layer between the cathode and beam plasmas . . . . . . . . . . . . . . . . 130

8.17 Average near-field plume properties as a function of cathode position. Error bars represent standard deviations of the averaged quantities. . . . 132

8.18 Comparison of the average near-field plasma potential to the beam divergence efficiency . . . . . . . . . . . . . . . 134

8.19 The electric field for $\dot{m}_{c}=10 \mathrm{SCCM}$ with the cathode at 60 and $120 \mathrm{~mm}$ on the OOP configuration. The magnitude of the field is plotted in a log scale on the color map. The arrows show the direction of the field. . . . 135

8.20 Correlation plots for select variables . . . . . . . . . . . . . . . . 139

9.1 Direct comparison of thruster performance when running with the OOP and EOP at identical operating conditions . . . . . . . . . . . . 144

9.2 Comparison of the internal magnetic fields of the thruster with the original and extended outer pole. . . . . . . . . . . . . . . . 146

10.1 Effect of drawn out plasma potential on efficiency components . . . . 152

10.2 Electron flux densities assuming classical mobility for the OOP with $\dot{m}_{c}=10 \mathrm{SCCM}$ and $r=120 \mathrm{~mm} \ldots \ldots \ldots \ldots 153$

10.3 Electron flux densities assuming classical mobility for the OOP with $\dot{m}_{c}=10 \mathrm{SCCM}$ and $r=50 \mathrm{~mm} \ldots \ldots \ldots \ldots 154$

B.1 Effect of probe heating on double probe traces . . . . . . . . . . 180

B.2 Effect of probe heating on measurement of plasma parameters . . . . 181

B.3 Animation of the probe traces at 10x speed . . . . . . . . . 181

C.1 Plasma properties for Kr with the OOP with $\dot{m}_{c}=2 \mathrm{SCCM}, r=50 \mathrm{~mm} 186$

C.2 Plasma properties for Kr with the OOP with $\dot{m}_{c}=2 \mathrm{SCCM}, r=60 \mathrm{~mm} 187$

C.3 Plasma properties for Kr with the OOP with $\dot{m}_{c}=2 \mathrm{SCCM}, r=80 \mathrm{~mm} 188$

C.4 Plasma properties for Kr with the OOP with $\dot{m}_{c}=2$ SCCM, $r=100 \mathrm{~mm} 189$

C.5 Plasma properties for Kr with the OOP with $\dot{m}_{c}=5 \mathrm{SCCM}, r=50 \mathrm{~mm} 190$ 
C.6 Plasma properties for $\mathrm{Kr}$ with the OOP with $\dot{m}_{c}=5 \mathrm{SCCM}, r=60 \mathrm{~mm} 191$

C.7 Plasma properties for Kr with the OOP with $\dot{m}_{c}=5 \mathrm{SCCM}, r=80 \mathrm{~mm} 192$

C.8 Plasma properties for Kr with the OOP with $\dot{m}_{c}=5 \mathrm{SCCM}, r=100 \mathrm{~mm} 193$

C.9 Plasma properties for Kr with the OOP with $\dot{m}_{c}=5 \mathrm{SCCM}, r=120 \mathrm{~mm} 194$

C.10 Plasma properties for Kr with the OOP with $\dot{m}_{c}=5 \mathrm{SCCM}, r=200 \mathrm{~mm} 195$

C.11 Plasma properties for Kr with the OOP with $\dot{m}_{c}=10 \mathrm{SCCM}, r=50 \mathrm{~mm} 196$

C.12 Plasma properties for Kr with the OOP with $\dot{m}_{c}=10 \mathrm{SCCM}, r=60 \mathrm{~mm} 197$

C.13 Plasma properties for Kr with the OOP with $\dot{m}_{c}=10 \mathrm{SCCM}, r=80 \mathrm{~mm} 198$

C.14 Plasma properties for Kr with the OOP with $\dot{m}_{c}=10 \mathrm{SCCM}, r=100 \mathrm{~mm} 199$

C.15 Plasma properties for Kr with the OOP with $\dot{m}_{c}=10 \mathrm{SCCM}, r=120 \mathrm{~mm} 200$

C.16 Plasma properties for Kr with the OOP with $\dot{m}_{c}=10 \mathrm{SCCM}, r=200 \mathrm{~mm} 201$

C.17 Plasma properties for Kr with the EOP with $\dot{m}_{c}=2 \mathrm{SCCM}, r=50 \mathrm{~mm} 204$

C.18 Plasma properties for Kr with the EOP with $\dot{m}_{c}=2 \mathrm{SCCM}, r=60 \mathrm{~mm} 205$

C.19 Plasma properties for Kr with the EOP with $\dot{m}_{c}=2 \mathrm{SCCM}, r=80 \mathrm{~mm} 206$

C.20 Plasma properties for Kr with the EOP with $\dot{m}_{c}=2 \mathrm{SCCM}, r=100 \mathrm{~mm} 207$

C.21 Plasma properties for Kr with the EOP with $\dot{m}_{c}=2 \mathrm{SCCM}, r=120 \mathrm{~mm} 208$

C.22 Plasma properties for Kr with the EOP with $\dot{m}_{c}=5 \mathrm{SCCM}, r=50 \mathrm{~mm} 209$

C.23 Plasma properties for Kr with the EOP with $\dot{m}_{c}=5 \mathrm{SCCM}, r=60 \mathrm{~mm} 210$

C.24 Plasma properties for Kr with the EOP with $\dot{m}_{c}=5 \mathrm{SCCM}, r=80 \mathrm{~mm} 211$

C.25 Plasma properties for Kr with the EOP with $\dot{m}_{c}=5 \mathrm{SCCM}, r=100 \mathrm{~mm} 212$

C.26 Plasma properties for Kr with the EOP with $\dot{m}_{c}=5 \mathrm{SCCM}, r=120 \mathrm{~mm} 213$

C.27 Plasma properties for Kr with the EOP with $\dot{m}_{c}=5 \mathrm{SCCM}, r=200 \mathrm{~mm} 214$

C.28 Plasma properties for Kr with the EOP with $\dot{m}_{c}=10 \mathrm{SCCM}, r=50 \mathrm{~mm} 215$

C.29 Plasma properties for Kr with the EOP with $\dot{m}_{c}=10 \mathrm{SCCM}, r=60 \mathrm{~mm} 216$

C.30 Plasma properties for Kr with the EOP with $\dot{m}_{c}=10 \mathrm{SCCM}, r=80 \mathrm{~mm} 217$

C.31 Plasma properties for $\mathrm{Kr}$ with the EOP with $\dot{m}_{c}=10 \mathrm{SCCM}, r=100 \mathrm{~mm} 218$

C.32 Plasma properties for $\mathrm{Kr}$ with the EOP with $\dot{m}_{c}=10 \mathrm{SCCM}, r=120 \mathrm{~mm} 219$

C.33 Plasma properties for Kr with the EOP with $\dot{m}_{c}=10 \mathrm{SCCM}, r=200 \mathrm{~mm} 220$

C.34 Plasma properties for Xe with $\dot{m}_{c}=2$ SCCM, $r=60 \mathrm{~mm}$. . . . . . . . 222

C.35 Plasma properties for Xe with $\dot{m}_{c}=2$ SCCM, $r=80 \mathrm{~mm}$. . . . . . . 223

C.36 Plasma properties for Xe with $\dot{m}_{c}=2$ SCCM, $r=120 \mathrm{~mm}$. . . . . . . 224

C.37 Plasma properties for Xe with $\dot{m}_{c}=5$ SCCM, $r=60 \mathrm{~mm}$. . . . . . . . 225

C.38 Plasma properties for Xe with $\dot{m}_{c}=5$ SCCM, $r=80 \mathrm{~mm}$. . . . . . . 226

C.39 Plasma properties for Xe with $\dot{m}_{c}=5$ SCCM, $r=120 \mathrm{~mm}$. . . . . . . 227

C.40 Plasma properties for Xe with $\dot{m}_{c}=10 \mathrm{SCCM}, r=60 \mathrm{~mm}$. . . . . . . 228

C.41 Plasma properties for Xe with $\dot{m}_{c}=10$ SCCM, $r=80 \mathrm{~mm} \ldots . .229$

C.42 Plasma properties for Xe with $\dot{m}_{c}=10$ SCCM, $r=120 \mathrm{~mm}$. . . . . . 230

D.1 Thruster performance as a function of cathode position. . . . . . . . 232

D.2 Current densities for select cathode positions . . . . . . . . . 233 
D.3 Ion energy distributions and ion current density as a function of angle for cathode mass flow rates of $2 \mathrm{SCCM} \ldots 2 . \ldots . \ldots 25$

D.4 Ion energy distributions and ion current density as a function of angle for cathode mass flow rates of $5 \mathrm{SCCM} \ldots . . \ldots 236$

D.5 Ion energy distributions and ion current density as a function of angle for cathode mass flow rates of $10 \mathrm{SCCM}$. . . . . . . . . . . 237

D.6 Efficiency decomposition versus cathode radial location . . . . . . . . 238 


\section{List of Tables}

7.1 Cathode Position Tests ................. 81

8.1 Efficiency loss mechanisms . . . . . . . . . . . . . . 116

8.2 Correlation coefficients between all single-point values . . . . . . . 137

E.1 Scalar data from separatrix crossing experiment . . . . . . . . . . . 242 


\section{Nomenclature}

$a_{n} \quad$ Adjustable fit parameter, page 57

$A_{\text {probe }}$ Probe area, page 48

B, $B \quad$ Magnetic field vector, magnitude, page 8

$D_{\|}, D_{\perp}$ Components of the electron diffusion coefficient parallel and perpendicular to B-field, page 12

E, $E \quad$ Electric field vector, magnitude, page 9

E Beam energy (eV), page 52

e Elementary charge, page 8

$f \quad$ The ion energy distribution function (IEDF), page 117

$f(v)$ Isotropic velocity distribution function, page 9

$h_{\text {coil }}$ Height of the coil cross-section, page 67

I Langmuir probe current, page 59

$I_{d} \quad$ Anode supply current, page 15

$I_{\text {mag }}$ Current through magnet coils, page 67

$I_{\text {sat }} \quad$ Ion saturation current, page 56

J Current density, page 67

J Current density, page 48

$J_{\text {mag }} \quad$ Current density in the magnet coils, page 67

$k \quad$ Boltzmann constant, page 9 
$m \quad$ Mass of a particle, page 8

$\dot{m} \quad$ Propellent mass flow rate, page 15

$\dot{m}_{a} \quad$ Anode mass flow rate, page 16

$\dot{m}_{c} \quad$ Cathode mass flow rate, page 16

$N_{\text {turns }}$ Number of turns on each magnet coil, page 67

$P \quad$ Input power, page 15

$P_{d} \quad$ Anode supply power, page 15

$q \quad$ Charge number, page 8

$r_{L} \quad$ Larmor radius, page 8

$r_{\text {offset }} x$ and $y$ offset of the magnet pole from the center of the thruster, page 67

$r_{p} \quad$ Langmuir probe radius, page 47

$T \quad$ Thrust, page 15

Te Electron temperature, page 9

$\mathbf{v}, v \quad$ Velocity, magnitude of velocity (speed), page 9

$V \quad$ Voltage applied to probe, page 57

V Voltage, page 117

$v \quad$ Ion velocity, page 116

$v_{\perp} \quad$ Velocity perpendicular to a magnetic field line, page 8

$V_{\text {cg }}$ Cathode coupling voltage: the voltage at which the cathode floats below ground, page 239

$V_{d} \quad$ Anode supply voltage, page 15

$\mathbf{v}_{\mathrm{gc}} \quad$ Velocity of the guiding center of a particle, page 9

$w_{\text {coil }}$ Width of the coil cross-section (Wire only. Does not include the iron core.), page 67

$\langle x\rangle \quad$ The expectation (average) value of any quantity $x$, page 117 
$\eta \quad$ Thruster efficiency, page 15

$\Gamma_{e} \quad$ Electron flux, page 13

$\eta_{\theta} \quad$ Beam divergence efficiency, page 116

$\eta_{I} \quad$ Current utilization efficiency, page 116

$\eta_{V} \quad$ Voltage utilization efficiency, page 116

$\eta_{\mathrm{Vcg}}$ Cathode coupling efficiency, page 116

$\eta_{\mathrm{vdf}} \quad$ Velocity distribution efficiency, page 116

$\lambda_{d} \quad$ Debye length, page 47

$\mu_{\|}, \mu_{\perp}$ Components of electron mobility coefficient parallel and perpendicular to the B-field, page 12

$\xi \quad$ Non-dimensional Langmuir probe radius, page 47

$\sigma \quad$ Collision cross-section, page 10

$v \quad$ Collision rate, page 10

$\langle\sigma v\rangle$ Collision rate coefficient, page 10

$\chi \quad$ Non-dimensional potential $\frac{e V}{k T_{e}}$, w.r.t. plasma potential, page 58

$\psi \quad$ Non-dimensional applied probe potential, page 58

$\psi_{n} \quad$ Non-dimensional potential of probe $n$ w.r.t. floating potential, page 58

$\Omega \quad$ Hall parameter, page 12

$\omega_{c e} \quad$ Electron-cyclotron frequency, page 12 
xxviii 


\section{Chapter 1}

\section{Introduction}

\subsection{Hall-Effect Thruster-Cathode Coupling and Perfor- mance}

Hall-effect thrusters (HETs) are a class of electric propulsion devices that use electric and magnetic fields to create a plasma and expel the ions at high velocity in order to generate thrust ${ }^{1}$ (Figure 1.1). A critical component of the HET is the cathode. The cathode is a plasma source that provides free electrons that serve two purposes. The first purpose is beam neutralization-sufficient electrons are expelled via the cathode to balance the charge emitted by the ion beam. The second purpose is to provide the "seed" electrons which initialize and sustain the plasma discharge near the exit plane of the HET. Detailed explanations of both HET and cathode are provided in Chapter 2.

The process by which electrons move from the cathode to the ion beam and anode of the thruster and how this process affects thruster performance is not well understood. Essentially, the process is the coupling between the cathode plasma and the main discharge plasma. Researchers have studied the effects of a variety of cathode parameters such as design ${ }^{2}$ and mass flow rate ${ }^{2,3}$ on HET performance. Furthermore, it has been repeatedly noted that cathode placement relative to the thruster has an effect on thruster performance. ${ }^{4,5,6,3,7,8}$ These studies' results will be further discussed in Chapter 3. What is clear from every study is that the choice of operation parameters of the cathode can significantly affect the efficiency with which an HET converts electrical power into thrust.

In particular, researchers at the Jet Propulsion Laboratory (JPL) have noted that thrusters with cathodes mounted in the center exhibit improved performance over those thrusters with cathodes in more traditional, external locations. ${ }^{5}{ }^{8}$ Unfortunately, centermounted cathodes are only feasible in those HETs with sufficient room in the center magnet pole to allow for the placement of the cathode and still have sufficient iron 

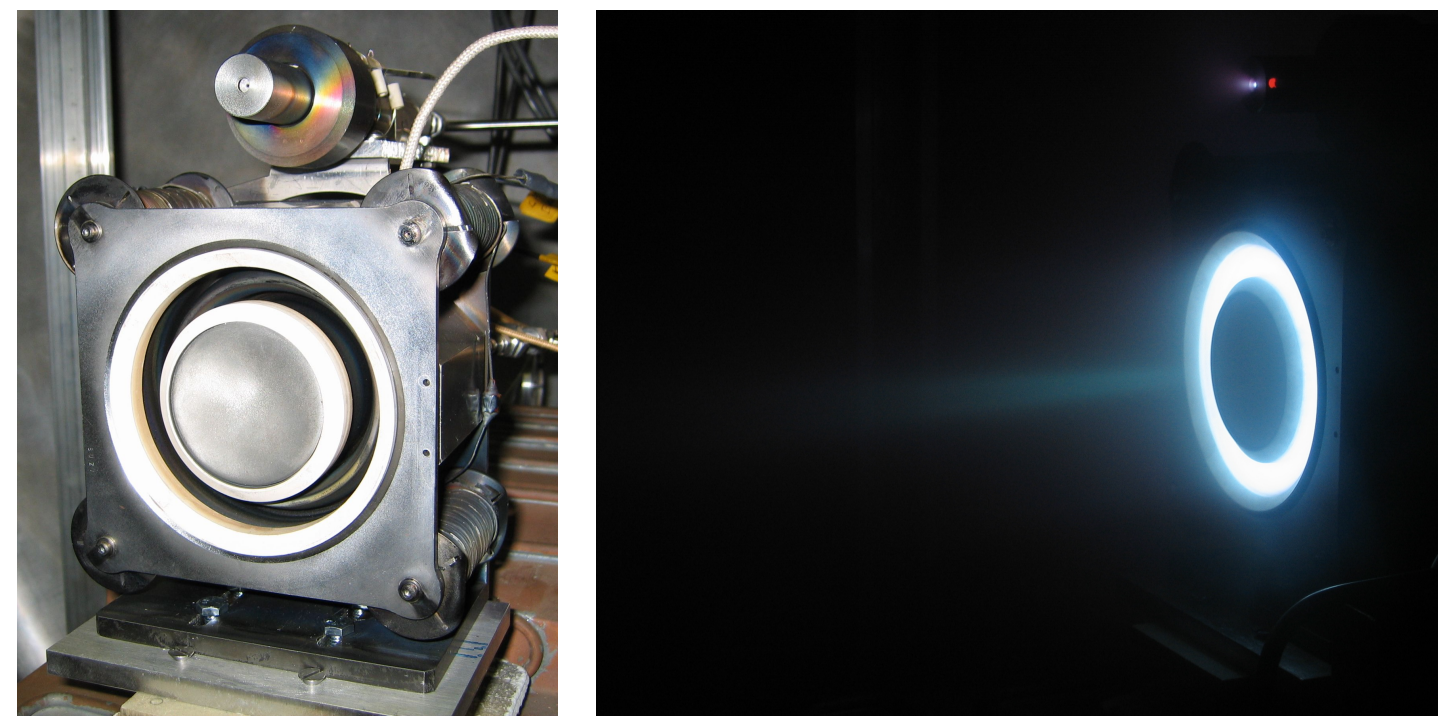

Figure 1.1: Photographs of an Aerojet BPT-2000 Hall-effect thruster

to make an appropriate magnetic circuit. HET cathodes are rarely smaller than $2 \mathrm{~cm}$ in diameter, and this puts a lower bound on the size of the thruster that can support a central-mount cathode. Smaller thrusters, such as Busek's BHT-200, which was recently flown on TacSat- $2^{9}$ (Figure 1.2), simply do not have sufficient room in their inner core for the cathode to be mounted internally. Therefore, externally mounted cathodes are still necessary on smaller thrusters. Furthermore, JPL's research into internally mounted cathodes is still preliminary. They have not compared thruster performance of an internally mounted cathode to an optimized external position, nor is there presently any proven theory as to why the internal cathode performs better.

Trial-and-error methods for determining the optimal cathode operation parameters are expensive and time consuming. While measuring the performance of the thruster, the cathode parameters must be adjusted. This is particularly difficult for cathode placement, as it may require multiple tests, with the cathode repositioned in between each one. With xenon costing \$50/L (in 2008), and the expense of staffing and operating a large vacuum chamber such as NASA-Glenn's VF-5 at $\$ 15,000$ per week, ${ }^{10}$ the cost of a full optimization study could be $\$ 100,000$. An improved understanding of the coupling processes will enable the parameter space that needs to be explored to be greatly reduced, thereby saving time and money in future HET development.

Furthermore, increased understanding of the interactions between the cathode plasma and the magnetic field topology will aid the design of HET magnetic field circuits. Cur- 
rently, the position of the cathode is an afterthought. The magnetic circuit is designed to optimize the generation of the ion beam. However, the inefficiencies associated with cathode coupling are not considered at this point. Only after the HET is built and tested is the position of the cathode considered. By understanding the effect of the magnetic field topology on cathode coupling, the magnetic circuit can be designed to simultaneously optimize the internal field, for the generation of the ion beam, and the external field, for good cathode coupling.

\subsection{Contribution of this Work}

The goal of this research is to improve the understanding of processes by which the cathode plasma couples to the main discharge of an HET. This coupling affects the performance of the thruster in multiple, interacting ways. In particular, this research helps illuminate the effect of cathode position and magnetic field topology on this coupling. Furthermore, it describes the multiple ways in which cathode position affects the performance

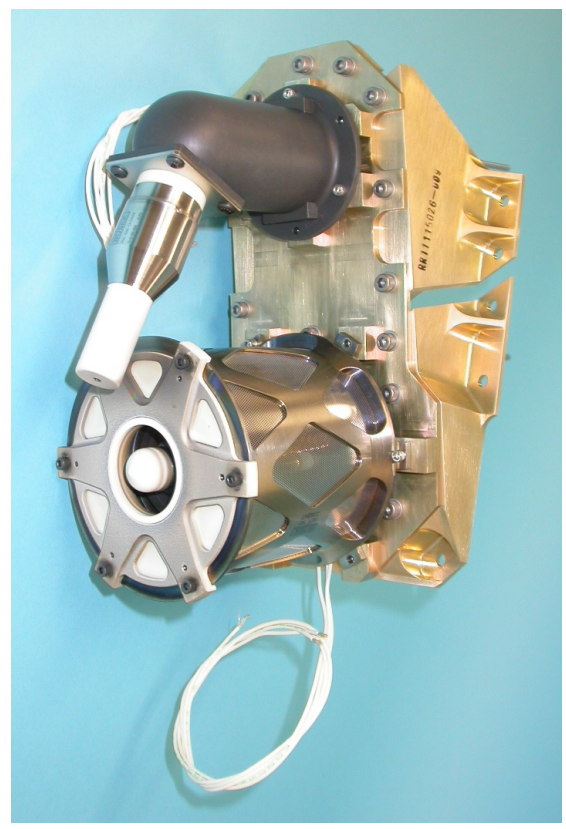

Figure 1.2: The Busek BHT-200 Halleffect thruster. (Photograph courtesy of Busek Co. Inc. See Appendix F) of the Hall thruster. In addition to the increased understanding of the plasma interactions and their effect on HET performance, this research should assist in the optimization of cathode position for flight thrusters. Improved understanding of the coupling will allow researches to know a priori which cathode positions are likely to be good, and which positions can be immediately ruled out.

To accomplish the goals of this research, several experiments were conducted. The experiments consisted of measuring the performance, ion beam characteristics, and near-field plume properties of a Hall-effect thruster while adjusting the cathode position and mass flow rate. The ion beam characteristics (ion beam current, ion beam density, and ion energy distribution functions) allow the determination of which efficiency loss mechanisms are being affected by the change in cathode position. The near-field plume properties enable us to see, at least partially, how the changes in beam properties are coming about, and how the cathode position is causing these changes.

The results have been compared to the external magnetic field of the thruster, and clearly show the important role this field plays in the coupling process. In particular, a previously unidentified (though perfectly obvious) structure known as the "magnetic field separatrix" is shown to be of significant importance, roughly dividing space 
into good performance and bad performance regions, which are easily identifiable with knowledge of the thruster's external magnetic field.

\subsection{Outline}

Following this introduction, Chapters 2 and 3 provide background material and a review of prior work in this field. Chapter 4 outlines the experimental methods used in all of the experiments conducted in this work. It is followed by Chapters 5 and 6 which expand on the some of the more complex methods and theories used. Chapter 7 describes preliminary experiments that were conducted in early 2007 . These early results motivated the experiments presented in Chapters 8 and 9. The work ends with synthesis, conclusions and suggestions for future work in Chapter 10. Additional supporting data are presented in the appendices.

\section{References for Chapter 1}

[1] Jahn, R. G., Physics of Electric Propulsion, McGraw-Hill Series in Missile and Space Technology, McGraw-Hill Book Company, New York, 1968.

[2] Albarède, L., Lago, V., Lasgorceix, P., Dudeck, M., Burgova, A., and Malik, K., "Interaction of a Hollow Cathode Stream with a Hall Thruster," 28th International Electric Propulsion Conference, Toulouse, France, March 17-21, 2003, Paper No. 03-333.

[3] Tilley, D. L., de Grys, K. H., and Myers, R. M., "Hall thruster-cathode coupling," 35th AIAA/ASME/SAE/ASEE Joint Propulsion Conference, June 20-24, 1999, Paper No. AIAA-99-2865.

[4] Hofer, R. R. and Gallimore, A. D., "Recent Results From Internal and Very-NearField Plasma Diagnostics of a High Specific Impulse Hall Thruster," 28th International Electric Propulsion Conference, March 17-21, 2003, Paper No. IEPC2003-037.

[5] Hofer, R. R., Johnson, L. K., Goebel, D. M., and Fitzgerald, D. J., "Effects of an Internally-Mounted Cathode on Hall Thruster Plume Properties," 42nd AIAA/ASME/SAE/ASEE Joint Propulsion Conference, July 9-12, 2006, Paper No. AIAA2006-4482.

[6] Beal, B. E., Gallimore, A. D., and Hargus, W. A., "The Effects of Cathode Configuration on Hall Thruster Cluster Plume Properties," 41 st AIAA/ASME/SAE/ASEE Joint Propulsion Conference, July 10-13, 2005, Paper No. AIAA-2005-3678. 
[7] Walker, M. L. R. and Gallimore, A. D., "Hall Thruster Cluster Operation with a Shared Cathode," Journal of Propulsion and Power, Vol. 23, No. 3, May 2007, pp. 528-536.

[8] Jameson, K. K., Goebel, D. M., Hofer, R. R., and Watkins, R. M., "Cathode Coupling in Hall Thrusters," 30th International Electric Propulsion Conference, Florence, Italy, September 17-20 2007, Paper No. IEPC-2007-278.

[9] Busek Company, I., "First US Hall Thruster Operational in Space," Press Release, 2007.

[10] Doehne, Sandra, Facilities Manager at NASA-Glenn Research Center, Personal communication (electronic mail), April 20, 2009. 


\section{Chapter 2}

\section{Background}

This chapter reviews basic knowledge necessary to understand the research presented in this work. It is not meant to be a comprehensive treatment of plasma physics or Hall-effect thruster (HET) dynamics. The discussion begins with a review of the motion of single electrons in electric and magnetic fields. From there it develops the bulk motion of electrons in a plasma. Armed with this knowledge, the reader is prepared for the explanation of HET operation which follows. After that, an phenomenological discussion of HET efficiency loss mechanisms is presented. The chapter concludes with an overview of cathode physics and an introduction to HET-cathode coupling.

\subsection{Electron Motion in Magnetized Plasmas}

The study of HET-cathode coupling is essentially a study of how one plasma, the cathode plasma, interacts with a second, the HET plasma. Even in plasmas of the lightest element, hydrogen, the electrons have roughly $1 / 2,000$ of the mass of the ions. In HET plasmas, which are typically heavy elements such as xenon (131.29 amu) the ratio is closer to $1 / 250,000$. Because of this, electric and magnetic fields within plasmas accelerate the electrons more rapidly.

Due to the high acceleration of the electrons relative to the ions, interactions between a plasma and its surroundings is usually governed by the motion of the electrons. In particular, because of their low mass, electrons respond strongly to the presence of a magnetic field, according to the Lorentz law, $\mathbf{F}=q \mathbf{v} \times \mathbf{B}$, whereas the ions pass through magnetic fields largely unaffected (at least across HET length and magnetic field scales). As will be shown, it is this very dichotomy that enables a Hall-effect thruster to operate. 


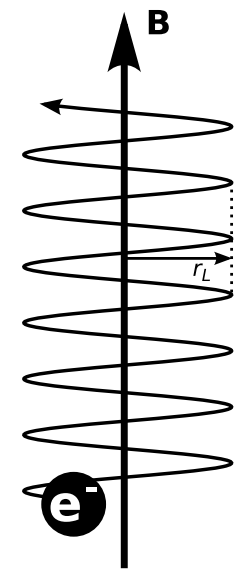

(a)

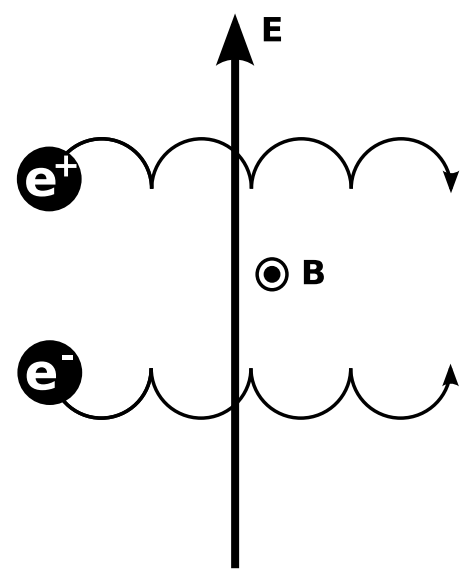

(b)

Figure 2.1: Motions of charged particles in (a) a constant magnetic field, and (b) crossed magnetic and electric fields

\subsubsection{Charged particle motion in a uniform magnetic field}

When a charged particle with some initial velocity enters a region of time-constant and uniform magnetic field, the Lorentz force causes it to turn about the magnetic field line, effectively trapping the particle on the line (see Figure 2.1a). The particle may freely move along the field line, potentially being accelerated by other external fields such as an electric field, but further motion perpendicular to the field line is limited to an orbit which is defined by the Larmor radius, given by

$$
r_{L} \equiv \frac{m v_{\perp}}{|q| e B}
$$

The Larmor radius provides an indication of how strongly a magnetic field traps a particle. The larger the radius, the less tightly the charge is bound to that field line.

Because electrons cannot easily cross magnetic field lines, but may freely travel along them, the magnetic field lines are electric equipotentials (at least to the extent that the electrons are completely unimpeded along the field line). The reason is simple: for electric fields parallel to a magnetic field lines, the electrons can freely move and redistribute along the field lines, thereby cancelling electric field. On the other hand, if one induces a field across the B-field lines, the mobility of the electrons is limited. The electrons are unable to move freely in the electric field to eliminate the potential difference, and the field persists. 


\subsubsection{Charged particle motion in crossed electric and magnetic fields}

If a uniform electric field is perpendicular to a uniform B-field, the particle no longer will be constrained to the field line. Instead, it will be accelerated by the E-field, until it reaches a sufficient velocity that the Lorentz force will turn it back in the opposite direction. E-field now slows the particle, bringing it to a stop in the direction parallel to the E-field, and then turning it back again. The net result of this motion is a drift in the overall motion of the particle in the direction perpendicular to both $\mathbf{E}$ and $\mathbf{B}$, the so-called $\mathbf{E} \times \mathbf{B}$ drift. This is illustrated in Figure 2.1b. The motion of the guiding center of the particle, i. e. its average motion, is given by

$$
\mathbf{v}_{\mathrm{gc} \perp}=\frac{\mathbf{E} \times \mathbf{B}}{B^{2}} .
$$

The astute reader will note that this motion of a particle's guiding center is independent of mass, and therefore appears to contradict my previous statements about ions being less affected than electrons by magnetic fields. Indeed, were both an ion and an electron in the same, constant fields, the motion of their guiding centers would be the same. However, the smallest Larmor radius for a xenon ion in typical HET conditions is on the order of $100 \mathrm{~mm}$ - roughly the same scale as the diameter and channel depth of the thruster. Furthermore, the B field will fall off rapidly as an ion travels this length in an HET system, thereby further increasing the Larmor radius and decreasing the trapping effect. This is quite beneficial since the goal of an HET is to create an ion beam, that is, to expel, rather than to trap, the ions.

\subsubsection{Bulk effects}

So far the discussion has focused on the motion of single electrons indpendent of interactions with other particles. However, plasmas are comprised of innumerable electrons. Often, these electrons have an isotropic, Maxwellian velocity distribution and the concept of temperature, in this case electron temperature, $T_{e}$, can be applied in the usual way:

$$
f(v) \propto v^{2} \exp \left(-\frac{m_{e} v^{2}}{2 k T_{e}}\right) .
$$

From this, we can define the average speed of a electron in such a thermal distribution

$$
v_{t h} \equiv \sqrt{\frac{2 k T_{e}}{m_{e}}} .
$$

We can substitute $v_{t h}$ for $v_{\perp}$ in Equation 2.1 to find the characteristic Larmor radius in a plasma of known species given $T_{e}$ and $B$. 
Not all plasmas have Maxwellian velocity distributions. In particular, low density plasmas in which the particle collision frequency is small compared to the time it takes a typical electron to transit the plasma may not reach thermodynamic equilibrium. This applies to many of the electrons in HET plasmas. However, the HET plume plasma is sufficiently Maxwellian such that the concept of electron temperature as a measure of mean electron speed is an acceptable approximation.

\subsubsection{Collisions}

Collisions between the electrons and the other particles in a plasma are of critical importance to understanding the dynamics of a plasma. To calculate frequency of a particular collision process, it is necessary to know the collision cross section, denoted by $\sigma$, the velocity distribution of the electrons, $f\left(v_{e}\right)$, and the density of targets. Under normal HET plasma conditions, the neutrals and ions can be considered stationary in comparison to the fast electrons. For a normalized, isotropic velocity distribution, $f$, the rate coefficient is given by:

$$
\left\langle\sigma v_{e}\right\rangle=\int_{0}^{\infty} \sigma\left(v_{e}\right) v_{e} f\left(v_{e}\right) d v_{e} .
$$

The product of the rate coefficient and the target density $\left(n_{0}, n_{i}\right)$ is the collision rate,

$$
v_{e}=n_{\mathrm{targets}}\left\langle\sigma v_{e}\right\rangle
$$

For electron-neutral collisions, the cross-sections are determined experimentally and given by the Siglo-Kinema database. ${ }^{1}$ For electron-ion collisions, which are Coulomb collisions, the cross-sections may be determined according to ${ }^{2}$

$$
\sigma_{\text {Coul }}=\frac{4 \pi}{9} \frac{e^{4} \log \Lambda}{\left(4 \pi \varepsilon_{0} T_{e}\right)^{2}},
$$

where $\log \Lambda$ is the Coulomb logarithm, given $b^{2}$

$$
\log \Lambda=\log \left[\frac{3}{2 \sqrt{\pi}} \frac{\left(4 \pi \varepsilon_{0} T_{e}\right)^{3 / 2}}{e^{3} n_{e}^{1 / 3}}\right] .
$$

Calculating the rate coefficients from Equation 2.7 and Equation 2.5 for a Maxwellian distribution is problematic because of the resulting integral diverges at the lower limit. For the present purposes, an approximation of the Coulomb rate coefficient given by 


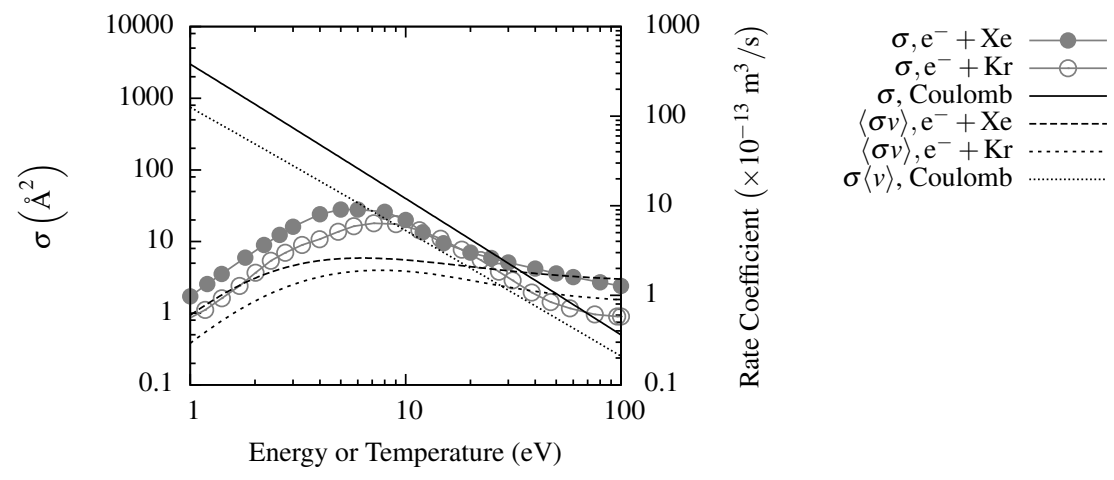

Figure 2.2: Electron-neutral momentum transfer and Coulomb collision cross sections and rate coefficients

$$
\left\langle\sigma_{\mathrm{Coul}} v\right\rangle \approx \sigma_{\mathrm{Coul}}\langle v\rangle
$$

will suffice. Here, $\langle v\rangle$ will be chosen as the thermal value of $\sqrt{k T_{e} / m_{e}}$.

Figure 2.2 compares the cross sections and rate coefficients for collisions with xenon neutrals, with krypton neutrals, and for Coulomb collisions (with either species of ion). Here Equation 2.7 and Equation 2.9 have been used for the Coulomb rate coefficients and the xenon and krypton rate coefficients have been calculated by numerically integrating the xenon and krypton cross sections for using Equation 2.5. Coulomb collisions are a weak function of $n_{e}$. A value of $1 \times 10^{17} \mathrm{~m}^{-3}$ has been chosen as representative of HET plasma.

In Figure 2.2 one sees that at high electron temperatures electron-neutral collisions dominate, whereas at lower temperatures the Coulomb collisions dominate. Typical HET plume plasma has a ratio of $n_{e} / n_{0} \approx 0.1$. And an $T_{e}$ of about $3 \mathrm{eV}$. In these conditions, the collision rates (not rate coefficients) are approximately equal.

\subsubsection{Electron diffusion and mobility}

Both Coulomb and neutral collisions are primarily elastic. Inelastic and ionizing collisions with neutrals have much smaller cross sections. Since the mass of an electron is so much smaller than that of an atom, electron-neutral collisions serve to randomize the direction in which the electron is traveling. Though for different reasons, Coulomb collisions also randomize the direction of electron travel. For an electron in a region of space free from magnetic fields, or for an electron traveling along a B-field line, these collisions impede the motion of the electron, and therefore reduce the mobility of the electrons. However, for an electron constrained to a magnetic field line, the randomizing collision provides an opportunity for an electron to cross field lines. If no electric field is present, this will cause the electron to undergo a random walk perpendicular to 
the B-field in addition to its free motion along the field. However, in the presence of a perpendicular electric field, the electron will move preferentially in the direction of the electric force.

This concept gives rise to the classical model of electron mobility. Mobility is a shorthand for the bulk motion of electrons in an electric field. Defining the mobility coefficient, $\mu$, and the diffusion coefficient, $D$, the bulk velocity of the electrons is given by: ${ }^{3}$

$$
\mathbf{v}_{\mathbf{e}}=-\mu \mathbf{E}-D \frac{\nabla n_{e}}{n_{e}} .
$$

The mobility and diffusion coefficients are, in turn, based on the Hall parameter, $\Omega$, which is a non-dimensional parameter which quantifies the extent to which a plasma is magnetized. Given the electron-cyclotron frequency, $\omega_{c e}=e B / m_{e}$, and the electron collision frequency, the Hall parameter is given by their ratio:

$$
\Omega=\omega_{c e} / v_{e}
$$

Because collisions impede motion along B-field lines and enable motion across Bfield lines mobility and diffusion coefficients are different depending on whether we are discussing electron motion parallel or perpendicular to the magnetic field.

$$
\begin{gathered}
\mu_{\|}=\frac{e}{m_{e} v_{e}} \\
\mu_{\perp}=\frac{\mu_{\|}}{1+\Omega^{2}} \\
D_{\|}=\frac{k T_{e}}{m_{e} v_{e}} \\
D_{\perp}=\frac{D_{\|}}{1+\Omega^{2}}
\end{gathered}
$$

In many regions of HET plasmas the Hall parameter is large compared to unity. This allows us, to simplify Equation 2.13 and Equation 2.15 to

$$
\begin{gathered}
\mu_{\perp} \approx \frac{m_{e}^{2} v_{e}}{e B^{2}}, \\
D_{\perp} \approx \frac{k T_{e} m_{e} v_{e}}{e^{2} B^{2}} .
\end{gathered}
$$

Comparing Equation 2.12 with Equation 2.16 and Equation 2.14 with Equation 2.17 and we see that collisions limit the motion along the B-field, while enabling motion across it.

Given the collision rates from Section 2.1.4, knowledge of the electron and neutral densities, (the ion density is also necessary, but typically $n_{i} \approx n_{e}$ ) and knowledge of 
the electric field, the electron flux density components relative to the B-field may be calculated:

$$
\begin{gathered}
\Gamma_{e, \|}=-E_{\|} \mu_{\|} n_{e}-D_{\|} \nabla_{\|} n_{e}, \\
\Gamma_{e, \perp}=-E_{\perp} \mu_{\perp} n_{e}-D_{\perp} \nabla_{\perp} n_{e} .
\end{gathered}
$$

In HETs, classical mobility is not the only process by which electrons cross magnetic field lines. ${ }^{4}$ It is thought that fluctuations in the plasma or collisions with walls increase the mobility, and some measurements ${ }^{5}$ have suggested that the mobility is closer to a fluctuation-induced Bohm mobility, where $\mu_{\perp} \approx 1 /(16 B) .{ }^{6}$ Regardless of the exact mobility mechanism, however, it remains true that magnetic fields impede the motion of electrons across field lines.

\subsection{Hall-Effect Thrusters}

\subsubsection{Basic mechanics}

With a basic understanding of the relevant plasma physics, we can now discuss the operation of a Hall-effect thruster. This thruster is a class of electric thruster which uses electric and magnetic fields to generate a plasma and expel the ions to generate thrust. While this class of thruster uses magnetic fields, they are considered electrostatic thrusters, rather than electrodynamic thrusters because the magnetic field is timeconstant, and enables the generation of thrust, but does not itself generate thrust. This is in contrast to true electrodynamic thrusters such as magnetoplasmadynamic (MPD) thrusters, and pulsed plasma thrusters (PPTs). A detailed description of HETs as well other types of electric propulsion as can be found in the classic text by Jahn. ${ }^{7}$

A simplified schematic of a Hall thruster is shown in Figure 2.3. The cathode generates a plasma which is a source of free electrons. The details of a cathode's operation will be discussed Section 2.3. The cathode is placed somewhere outside of the main discharge chamber. The anode is situated in the rear of the HET and is biased to between $100 \mathrm{~V}$ and $1000 \mathrm{~V}$ relative to the cathode. ${ }^{8,9,10,11,12,13}$ Neutral gas is introduced by a diffuser in or near the anode. Free electrons from the cathode initiate the second, main discharge plasma.

At this point in the description there are two plasmas, but no acceleration of the ions and no thrust. To achieve thrust, a magnetic field is placed near the exit such that the field lines cross the channel, roughly parallel to the exit plane. This magnetic field impedes the motion of the electrons across the channel to the point where they are considered trapped. Meanwhile the $\mathbf{E} \times \mathbf{B}$ drift causes them to rotate azimuthally 


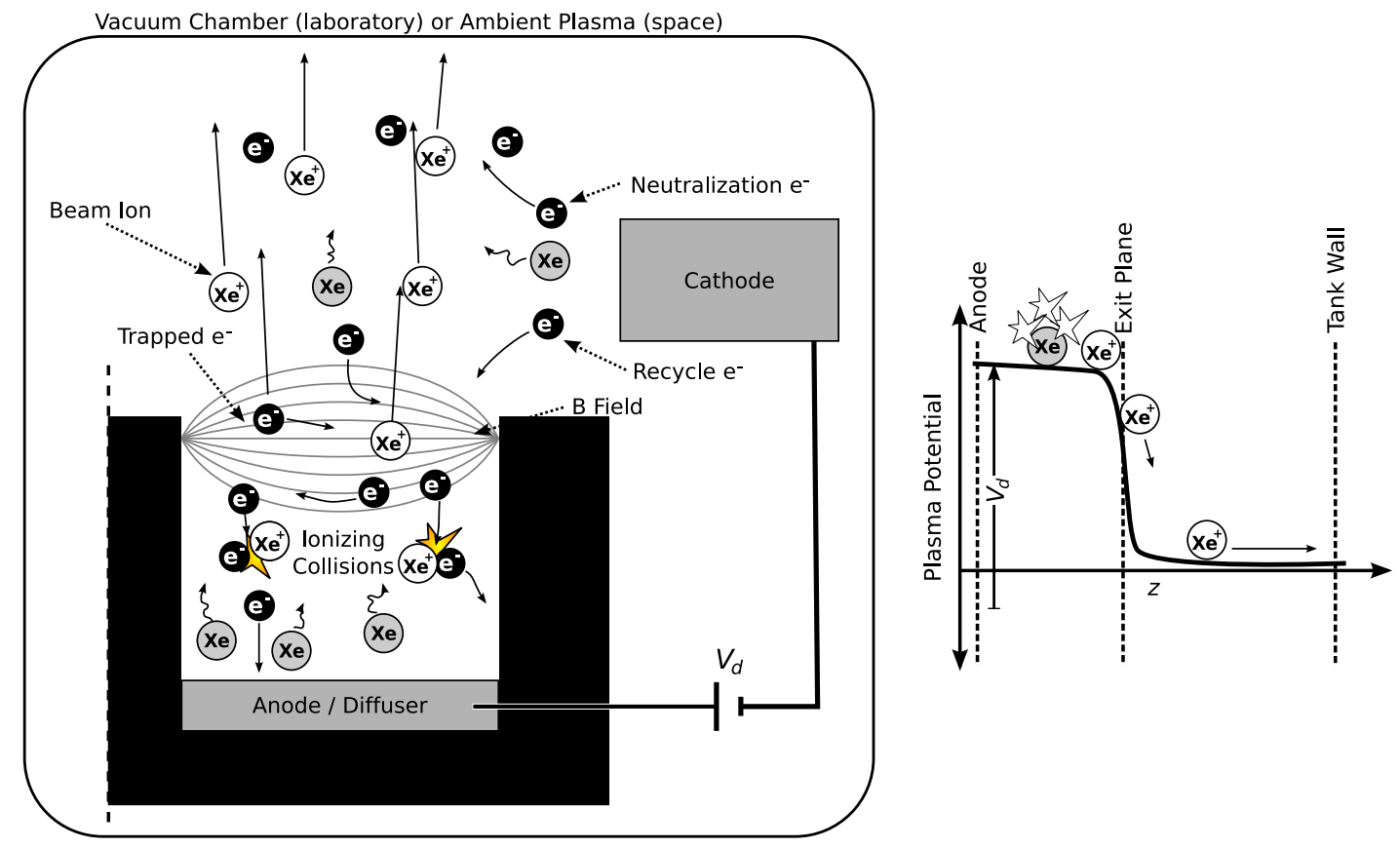

Figure 2.3: (Left) Simplified schematic of Hall-effect thruster discharge chamber and associated processes (Right) An indication of the local plasma potential along the channel centerline 
around the thruster, generating the "Hall current" from which the thruster takes its name.

This trapped population of electrons generates a highly negatively charged (relative to the anode) region of space near the exit plane of the channel. Thus a strong electric field or "potential hill" is created in the region of high magnetic field. This potential hill is typically $70-90 \%$ of the discharge voltage, the potential applied between the anode and the cathode. As collisions (and possibly other processes) cause electrons to exit the trap the electrons are accelerated toward the anode, gaining enough energy to ionize the neutrals on the upstream side of the magnetic trap. Because of the high energy and density of electrons in this region, most of the ionization in the system occurs here. Barring a charge-exchange collision, wall collision or other recombination event, an ion created in the upstream of the magnetic trap are accelerated by the local electric field, gaining significant energy as they cross the potential hill associated with the magnetic trap. Recall that, unlike the electrons, the trajectories of the ions are not significantly affected by the magnetic field. Thus, the ions are accelerated out of the thruster and generate thrust.

In modern thrusters, the gas used is typically xenon. Xenon is chosen because its high mass, $131.29 \mathrm{amu}$, and low ionization potential, $12.1 \mathrm{eV}$, make it desireable. The low ionization potential means that less energy must be spent in creating the ion-electron pairs, and higher mass atoms are typically have larger ionization crosssections, making ionization more likely. Furthermore, xenon, being a noble gas, is safe to handle. However, it is quite expensive. As of this writing (2008) it is currently $\$ 50 / \mathrm{L}$. At usage rates on the order of $20 \mathrm{~L} / \mathrm{hr}$, testing can be prohibitively expensive for most research programs. Therefore, krypton is often used as an alternative propellent. Its lighter mass, $83.8 \mathrm{amu}$, and higher first ionization potential, $14.0 \mathrm{eV}$, make it less efficient than xenon, but it is significantly less expensive at $\$ 10 / \mathrm{L}$.

\subsubsection{Efficiency}

The electrical efficiency of a Hall thruster is the efficiency with which the device converts electrical power into thrust. Given the thrust $(T)$, mass flow rate $(\dot{m})$ and input power $(P)$, the efficiency is given by

$$
\eta=\frac{T^{2}}{2 \dot{m} P}
$$

Often only the anode (sometimes called "discharge") power is considered:

$$
P=P_{d}=I_{d} V_{d}
$$


The efficiency calculation will also vary depending on whether cathode mass flow rate is included. Typically, two efficiencies are shown in this document. The first is the "anode efficiency," which neglects cathode propellent,

$$
\eta_{a}=\frac{T^{2}}{2 \dot{m}_{a} I_{d} V_{d}},
$$

while the second, "total efficiency," includes it,

$$
\eta_{t}=\frac{T^{2}}{2\left(\dot{m}_{a}+\dot{m}_{c}\right) I_{d} V_{d}} .
$$

The term "total efficiency" is, perhaps, a bit misleading since certain other power sources are neglected. In particular, the power to the magnet coils, the cathode heater power, and, where applicable, the cathode keeper power are not included in these efficiency calculations. These have been left out since these parameters are more likely to be a function of the specific cathode or thruster, rather than a general feature of all thruster systems.

\subsubsection{Efficiency loss mechanisms}

There are several mechanisms by which efficiency is lost. The mathematical details will be presented later in Chapter 8. For now, phenomenological explanations will provide the reader with a basic understanding.

Because of the curvature of the magnetic fields which trap the electrons, and the fact that magnetic field lines are nearly equipotentials, the exit of an HET is an ion lens. In other words, the ions are not only accelerated, but they are also redirected or focused. One of the biggest challenges in HET design is to achieve the proper focusing. An unfocused beam expels many ions in a direction not parallel to the thrust axis. Due to the cylindrical symmetry of the system, any ion expelled at one angle, will, on average, have an ion expelled at the opposite angle. The net result is that the perpendicular components of the thrust generated by these two ions cancels, leaving only the parallel components. However, it cost the same amount of energy to send the ions on diverging paths as it would have to send them both parallel to the thrust axis. Thus, beam divergence represents an efficiency loss mechanism, as illustrated in Figure 2.4a.

A second efficiency loss mechanism is known as voltage utilization, and it is illustrated in in Figure 2.4b. There are two reasons for the mechanism. First, as the preceding description of HET operation states, most of the ionization occurs upstream of the potential hill. However, ionization can take place anywhere in the system, and in particular, may be created partway down the potential hill. When this happens, the ion does not receive the full amount of energy available. Rather, the ion shares the full 


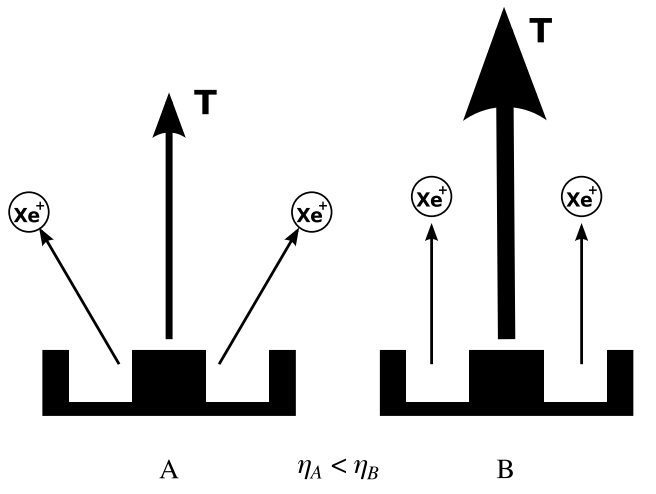

(a) Divergence

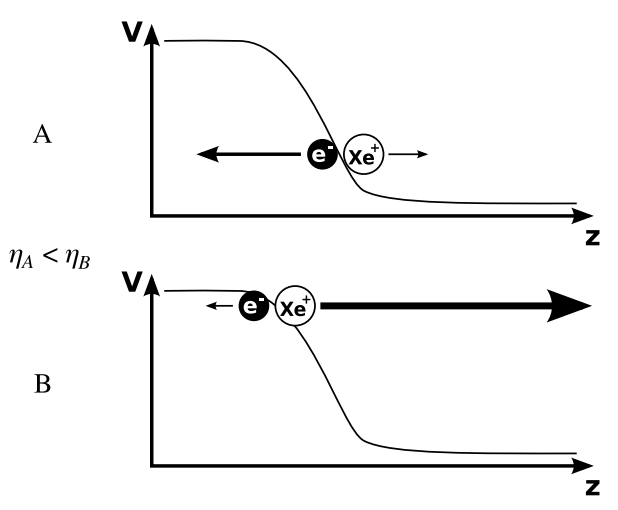

(b) Voltage utilization

Figure 2.4: Efficiency loss mechanisms in a Hall-effect thruster

energy with the freed electron, which will ultimately deposit its energy as heat in the anode, thus wasting it. Second, the bulk of the ionization can never occur at anode potential. An ionization event occurring at anode potential would leave the freed electron with zero potential energy. Thus, it would be unable to gain enough energy to ionize any more neutrals. If all ionization took place at this potential, cascade ionization could not happen. Therefore, voltage utilization efficiency accounts for the cost of ionization.

A third efficiency loss mechanism is the velocity distribution efficiency. It arises because thrust is linearly dependent on the velocity of the ions $(T \propto \dot{m} v)$ while the power required to accelerate the ions to the terminal velocity scales as velocity squared $(P \propto$ $\dot{m} v^{2}$ ). Therefore, it is more efficient to accelerate all of the particles to the same velocity than to accelerate particles to different velocities. To see why, imagine a thruster that expels two ions of mass $m$, as shown in Figure 2.4c. In Case A, the thruster accelerates one particle to a velocity $v$, while the second is accelerated to a velocity $3 v$. The total impulse delivered to the thruster is $4 m v$. In Case B, the thruster expels both particles with a velocity $2 v$, also yielding a total impulse of $4 m v$. Now let's examine the energy necessary to accelerate the particles. In the first case, the energy required is

$$
E_{A}=\frac{1}{2} m v^{2}+\frac{1}{2} m(3 v)^{2}=5 m v^{2} .
$$

Meanwhile, in the second case the energy required is

$$
E_{B}=2 \frac{1}{2} m(2 v)^{2}=4 m v^{2} .
$$




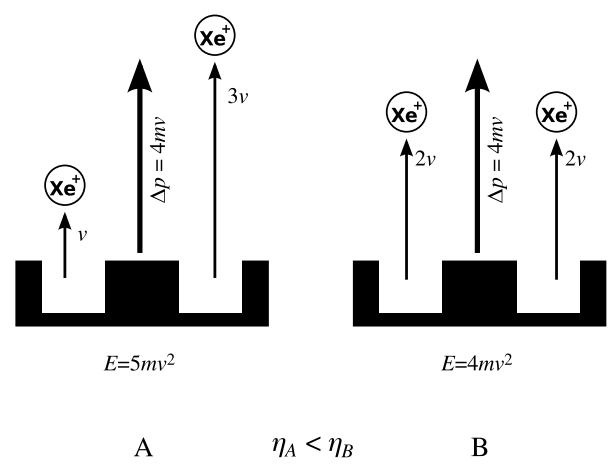

(c) Velocity Distribution

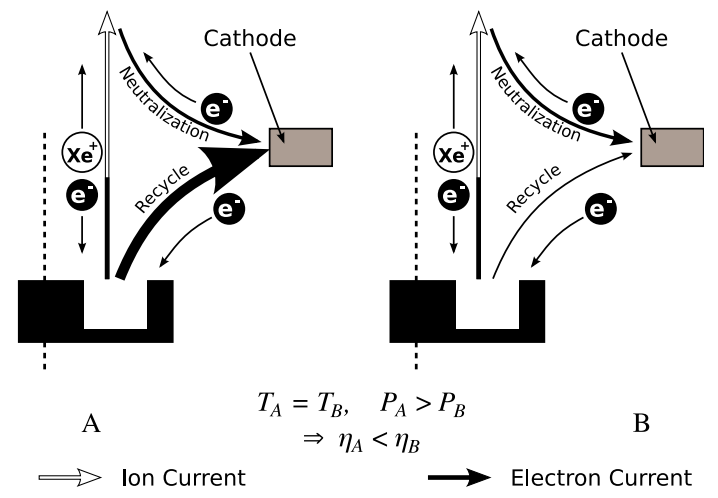

(d) Current Utilization

Figure 2.4: Efficiency loss mechanisms (continued)

Obviously there are more than two particles in a real thruster. Those who are interested can further inspect Table 8.1 and Equation 8.11 to see that the assertion that narrow velocity distributions are more efficient extends to all situations.

A fourth efficiency loss mechanism is current utilization. In an ideal thruster, there would be exactly one electron emitted from the cathode for each singly-charged ion leaving the HET. The current from these electrons is the "neutralization current." The only electrons that would reach the anode would be those freed by ionization of the propellent. However, it is possible for some electrons to move from the cathode, through the magnetic trap, and to the anode directly. As already mentioned, this current is called the recycle current. It is an efficiency loss, since power is required to create that current, yet no thrust is generated. Figure 2.4d illustrates this efficiency loss mechanism.

In addition to these efficiency mechanisms, there is also an inefficiency associated with cathode coupling. It will be discussed in Section 2.4.

\subsection{Cathodes}

Having provided an overview of the physics behind HET operation, let us turn to the cathode, which, until now has been a magic plasma source. HET cathodes come in a variety of designs, but the most common is the orificed, thermionic hollow cathode (Figure 2.5). This cathode is comprised of a tube containing an electric heater and a low work function material such as barium-oxide $(\mathrm{BaO})$ impregnated tungsten or lanthanum-hexaboride $\left(\mathrm{LaB}_{6}\right)$. The tube is filled with a neutral gas, typically the same as the propellent for the HET, and a small orifice at one end allows the gas to escape into the surrounding space. The low-work-function material, also known as the emitter, 


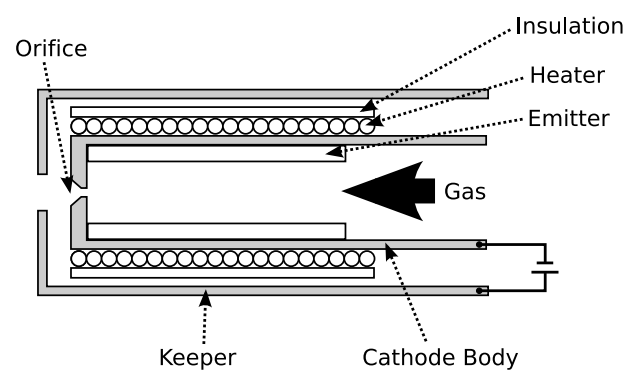

Figure 2.5: Cross-section of a typical orificed hollow cathode

is situated near this exit, and is heated by the nearby electric heater. The emitter is biased negative relative to an anode, which is situated outside of the exit of the cathode. This anode may be the HET anode, but more typically, a secondary anode called the "keeper" is positioned much closer to the cathode orifice, and it is particularly important during cathode start-up.

With the potential between the keeper and the emitter set to something on the order of $300 \mathrm{~V}$ and the neutral gas flowing, heat is applied to the emitter, which enables some of the electrons to tunnel out of the emitter material into the surrounding space. Once the electrons are free of the emitter, they are accelerated by the electric fields set up by the potential between the keeper and the emitter. In the process, electrons collide with neutral atoms and ionize some of them. The electrons freed from ionized atoms are also accelerated by the same electric fields, and, in turn, ionize more neutrals. This cascade ionization creates the cathode plasma which diffuses into the surrounding space. Densities and temperatures depend on cathode design and mass flow rate. Typical densities in the cathode plume are $10^{16}$ to $10^{20} \mathrm{~m}^{-3}$ with electron temperatures of $2-3 \mathrm{eV} .{ }^{14}, 15,16$ Under these conditions, recombination is unlikely, and thus we have a plasma source from which free electrons may be readily taken.

In most thermionic cathodes, once the plasma discharge is created, the ions colliding with the emitter provide sufficient heat to the emitter that electrons continue to tunnel out, and the electric heater may be powered off. Furthermore, for a cathode in a Hall-effect thruster system, the keeper is set to a minimum current such that the cathode discharge is maintained should the main thruster discharge be extinguished. Thus, there is only one control on a cathode: the gas flow rate. However, some cathodes, including the one used in these experiments, do not have a sufficient heat load from the backstreaming ions to maintain the discharge, and the electric heater must be left on after the discharge is initiated, though typically at lower power levels than are necessary to start the cathode. For these types of cathodes, there are two adjustable parameters: flow rate and heater power. 


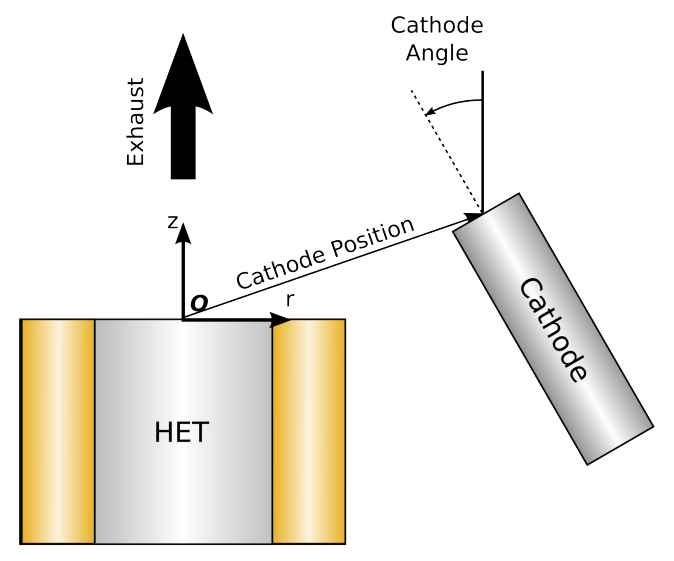

Figure 2.6: Coordinate system used to describe the cathode position

As a final note, the cathode used in this research is similar, but not identical to hollow cathodes discussed. Specifically, it is a thermionic cathode, but it is not orificed. However, at the level of the general description provided here, both the typical HET hollow cathode and the cathode used in these experiments are identical. The specific cathode used will be further discussed in Section 4.4.4.

\subsection{HET-Cathode Coupling}

The cathode should be placed so as to provide ideal coupling between the electrons of the cathode plasma and the main discharge plasma of the HET. To date, cathode placement has been largely done by trial and error, an expensive and time-consuming process. Typically, the cathode is placed radially outside of the main body of the HET. Often it is also placed slightly downstream of the exit plane, and angled in toward the thruster. Figure 2.6 shows a somewhat typical cathode location. (The cathode is positioned radially further away than is normal to provide sufficient space in the figure for the text labels.) Furthermore, the figure describes the basic coordinate system used in most cathode research, including this work. The origin of the coordinate system is at the intersection of the exit plane and the axis of the HET. The axial, $z$ coordinate increases into the plume of the HET. The radial, $r$ coordinate increases with distance from the axis. The center of the cathode orifice is the reference point for the cathode position. The angle the cathode makes with the thrust axis is the cathode angle, with positive angles denoting a cathode pointing toward the thrust axis.

In a Hall-effect thruster, the free electrons from the cathode are necessary for two functions. The first is beam neutralization. Sufficient electrons must be expelled from the cathode to balance the ions in the beam, otherwise the spacecraft would become 
negatively charged. Beyond this, a certain number of electrons must travel from the cathode to the ionization region of the thruster in order to initiate and sustain the discharge through collisional ionization of the neutral propellant. This extra current is called the "recycle current," because we consider these electrons to be repeatedly recycled through the system and never expelled into the beam.

Collisions between electrons and other particles, primarily neutrals, in the plasma impede the motion of the electrons from the cathode to the anode and beam. Furthermore, it is impractical to create the magnetic trap in the HET chamber without also generating a magnetic field outside of the thruster. An external cathode is placed in the external magnetic field, and that field impedes the motion of the cathode electrons. Because of the mechanisms that impede the motion of the electrons to the ion beam and anode, work is required simply to get the electrons to where they are needed for the plasma to maintain quasineutrality. This work is an efficiency loss mechanism as it does not directly generate thrust. The cathode coupling voltage provides a rough measure of the amount of work required to move the electrons from the cathode. In the laboratory, this is the potential at which the cathode floats below ground, and it is denoted by $V_{\text {cg. }}$. In space with negligible electric fields compared to that generated by the thruster, the ambient plasma potential serves the same function as ground.

To understand why this is the correct measure, consider an ion "falling" out of the thruster. While most of the potential drop occurs inside the thruster, there is some small drop all they way through the sheath at the tank wall at the end of the ion's trajectory. The ion is accelerated through this drop, and the opposite force pushes back on the thruster, generating the thrust. When the ion reaches the tank wall, it impacts the surface, grabs an electron, and exits the system as a neutral. In order to maintain charge neutrality, an electron from the cathode must also travel to the tank wall to make up for the electron that neutralized the ion.* The anode is biased at $V_{d}$ volts with respect to the cathode. Assuming no efficiency loss mechanisms affect the ionization and acceleration of the electron, the (singly-ionized) ion should hit the tank wall with $E_{d}=e V_{d}$ of energy unless it also requires some energy to move the electron from the cathode to the wall. If we denote this energy by $E_{\mathrm{cg}}$ then the energy that the ion receives can be no more than $E_{d}-E_{\mathrm{cg}}$, which implies that the ion starts at a potential of $V_{d}-V_{\mathrm{cg}}$ where $V_{\mathrm{cg}}=E_{\mathrm{cg}} / e$. Furthermore, since it requires $E_{\mathrm{cg}}$ of energy to move the electron to the wall, the cathode must be at a potential $V_{\text {cg }}$ relative to the tank wall.

Of course, the preceding analysis ignores many effects. The ion may not be ionized at the maximum potential. The ion may suffer a charge-exchange collision before reaching the tank wall. An ion and electron may recombine before reaching the wall.

${ }^{*}$ As previously noted, in space, the ambient plasma potential serves the same function as the ground of the tank wall. Recombination between the ion and electron are unlikely in this situation, but that is not relevant. Once the charges are no longer in an electric field generated by the thruster, they have exited the system. 


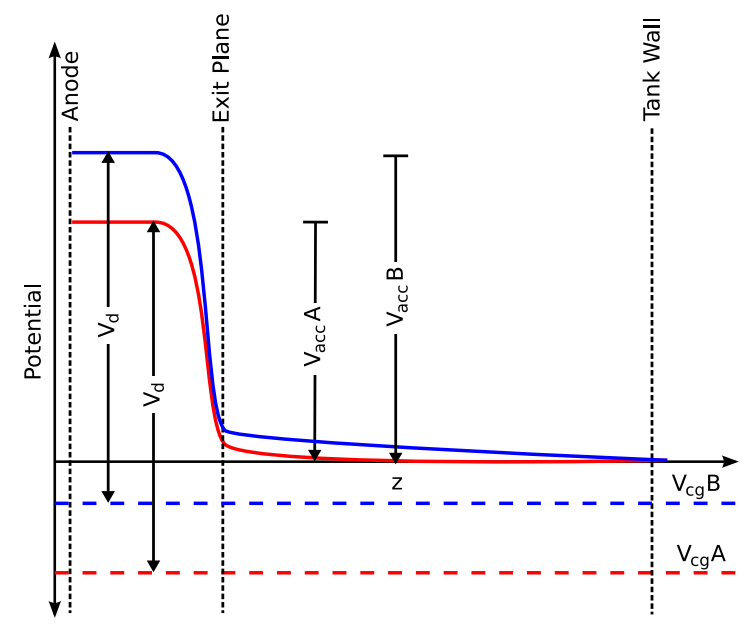

Figure 2.7: The effect of cathode coupling voltage on energy available for ion acceleration

However, none of these effects substantially change the picture that $E_{\mathrm{cg}}$ represents the average amount of energy necessary to move an electron from the cathode to where it needs to go for the thruster to operate. Since this energy is not propulsive (due to its small mass compared to the ion any thrust generated by the electrons is negligible) it is an efficiency loss mechanism. It arises because collisions, magnetic fields, and possibly other effects not considered here impede the motion of the electrons from the cathode.

Figure 2.7 illustrates this efficiency cost. In Case A, the cathode floats lower than in Case $\mathrm{B}$, that is, $V_{\mathrm{cg}} A<V_{\mathrm{cg}} B$. The maximum potential drop available to an ion is $V_{\mathrm{acc}}=$ $V_{d}-V_{\mathrm{cg}}$. Obviously, Case B with the higher $V_{\mathrm{cg}}$ has a higher $V_{\mathrm{acc}}$ and therefore more energy is available to the ions. Therefore, all other things being equal, the efficiency of Case B will be higher than that of Case A.

A semantic note: since cathode coupling voltage is a negative value, terminology can become confusing. For the sake of consistency, unless otherwise stated this document will refer to the actual value of the negative number, and not to its magnitude. Thus, a "high" $V_{\text {cg }}$ means one that is close to 0 , while a "low" $V_{\text {cg }}$ is more negative. Furthermore, high coupling voltages are desirable in that they are a smaller efficiency hit, per the preceding argument, while low cathode coupling voltages are undesirable.

\section{References for Chapter 2}

[1] Morgan, W. L., Boeuf, J. P., and Pitchford, L. C., "The Siglo Database, CPAT, and Kinema Software," http://www.siglo-kinema.com/database/index.htm, Accessed January 2009. 
[2] Fridman, A. and Kennedy, L. A., Plasma Physics and Engineering, Taylor \& Francis Group, 2004, p. 196.

[3] Chen, F. F., Introduction to Plasma Physics and Controlled Fusion, Plenum Press, New York, 1984, p. 158.

[4] Janes, G. S. and Lowder, R. S., "Anomalous Electron Diffusion and Ion Acceleration in a Low-Density Plasma," The Physics of Fluids, Vol. 9, No. 6, June 1966, pp. 1115-1123.

[5] Meezan, N. B., Hargus, W. A., and Cappelli, M. A., "Anomalous electron mobility in a coaxial Hall discharge plasma," Physical Review E, Vol. 63, February 2001, Paper No. 026410.

[6] Bohm, D., The Characteristics of Electrical Discharges in Magnetic Fields, McGraw-Hill, New York, 1949, p. 65.

[7] Jahn, R. G., Physics of Electric Propulsion, McGraw-Hill Series in Missile and Space Technology, McGraw-Hill Book Company, New York, 1968.

[8] Manzella, D. H. and Jacobson, D., "Investigation of Low-Voltage/High-Thrust Hall Thruster Operation," 39th AIAA/ASME/SAE/ASEE Joint Propulsion Conference, Huntsville, AL, July 20-23, 2003, Paper No. AIAA-2003-5004.

[9] Butler, G. W., Yuen, J. L., Tverdokhlebov, S. O., Semenkin, A. V., and Jankovsky, R. S., "Multimode, High Specific Impulse Hall Thruster Technology," 36th AIAA/ASME/SAE/ASEE Joint Propulsion Conference, Huntsville, AL, July 1719, 2000, Paper No. AIAA-2000-3254.

[10] Pote, B. and Tedrake, T., "Performance of a High Specific Impulse Hall Thruster," 27th International Electric Propulsion Conference, Pasadena, CA, October 15-19 2001, Paper No. IEPC-01-35.

[11] Hofer, R. R., Jankovsky, R. S., and Gallimore, A. D., "High-Specific Impulse Hall Thrusters, Part 1: Influence of Current Density and Magnetic Field," Journal of Propulsion and Power, Vol. 22, No. 4, July 2006, pp. 721-731.

[12] Hofer, R. R., Jankovsky, R. S., and Gallimore, A. D., "High-Specific Impulse Hall Thrusters, Part 2: Efficiency Analysis," Journal of Propulsion and Power, Vol. 22, No. 4, July 2006, pp. 732-740.

[13] Belikov, M. B., Gorshkov, O. A., and Rizakhanov, R. N., "Investigation of a Hall Thruster of $1.5 \mathrm{~kW}$ Class of Power At Specific Impulses Up to $3000 \mathrm{~s}$," 36th AIAA/ASME/SAE/ASEE Joint Propulsion Conference, Huntsville, AL, July 1619, 2000, Paper No. AIAA-2000-3253. 
[14] Csiky, G. A., "Langmuir Probe Measurements in a Discharge from a Hollow Cathode," Journal of Spacecraft and Rockets, Vol. 7, No. 4, April 1970, pp. 474-475.

[15] Goebel, D. M., Jameson, K. K., Watkins, R. M., Katz, I., and Mikellides, I. G., "Hollow cathode theory and experiment I: Plasma characterization using fast miniature scanning probes," Journal of Applied Physics, Vol. 98, No. 11, 2005, Paper No. 113302.

[16] Williams, G. J., Smith, T. B., Patrick, T. A., and Gallimore, A. D., "Characterization of the FMT-2 discharge cathode plume," 26th International Electric Propulsion Conference, Kitakyushu, Japan, 1999, Paper No. IEPC-99-104. 


\section{Chapter 3}

\section{Review of Prior Research}

This chapter provides a review of past and current research in the area of ion beam-cathode coupling. Early work in the subject is primarily focused on gridded ion thrusters, as development of this type of thruster, at least in the United States, predates work on HETs. Following the section on early work, the discussion will turn to more recent work on HET-Cathode coupling.

\subsection{Cathode Design}

Before discussing the process by which a cathode plasma couples to a thruster plasma, it is worth noting that there are multiple designs for cathodes, and that various parameters in the cathode design can affect the efficiency of the device itself, independent of the thruster or cathode position. The most common cathode design used in conjunction with both gridded ion thrusters and HETs is the orificed thermionic hollow cathode. The orificed hollow cathode design is superior to others in that a small plasma is confined to a small area. This minimizes the power requirements, and allows the device to be self-heating. ${ }^{1}$ Several studies have focused on designing these cathodes to achieve optimal performance from the cathode itself-that is, to minimize the power per unit of current delivered from the cathode in a cathode-keeper system. ${ }^{1,2,3}$ These studies primarily focus on modifying the dimensions of the orifice and the insert to achieve these goals.

In addition to self-heating cathodes, many facilities use "hot" cathodes- those which require additional heat to maintain the plasma. These cathodes are cheaper to purchase or manufacture and easier to maintain than their self-heating counterparts, but have the obvious disadvantage of requiring constant power to the heater. These cathodes operate in a similar fashion, but do not necessarily have an orificed emitter. The lack of an orifice allows more heat to dissipate which must be resupplied by the electric heater. These cathodes are not typically used in flight applications because of the extra power 
requirement. However, they are often cheap and easy to build, and thus make good laboratory cathodes.

When comparing one cathode coupling experiment to the next, it is necessary to remember that different cathode designs perform differently. Therefore, one cannot compare, say, the cathode coupling voltage from one experiment to another using a different cathode in any meaningful way. However, trends shown in an experiment are more likely to be universal.

\subsection{Early Work}

Beginning in 1961 and culminating with the flights of SERT I in 1964, and SERT II in 1969, the U.S. developed and flew its first electric propulsion thrusters. ${ }^{4}$ These thrusters were of the gridded ion variety, fueled by either cesium or mercury vapors. Despite the difference in thruster type and fuel from the xenon-fueled Hall-effect thrusters on which this research focuses, these early thrusters also employed hollow cathodes for ion beam neutralization, and some of the observations made on the coupling between the cathode and the gridded ion thruster will be later echoed by researchers focusing on HETs. Therefore, it is worthwhile to survey these works.

As part of the process of developing SERT II, Hall, Kemp and Shelton performed extensive studies on mercury hollow cathodes in an effort to optimize the design of the device and the overall thruster system. ${ }^{5}$ In their study, the authors note that the heating power delivered to the mercury reservoir-which directly corresponds to cathode gas flow rate- has a dramatic effect on the coupling efficiency. As the cathode was moved away from the acceleration grids, higher cathode mass flows were required to maintain a stable discharge. Similar trends in cathode position were reported in other work on these early neutralizers. ${ }^{6}$ Perhaps one of the most interesting conclusions of the research by Hall et al. is something which is now taken for granted-it is necessary to run the thruster with the cathode and anode isolated from the facility ground in order to gather any meaningful information about cathode operation.

Ward and King performed further work which focused on the effect of varying the flow rates of cathode vapor. ${ }^{7}$ This work clearly indicated what will be an oft-repeated theme-increasing the cathode flow rate causes an increase in cathode coupling voltage. As the cathode flow was increased from $2 \%$ of the anode mass flow to $90 \%, V_{\mathrm{cg}}$ increased from $-23 \mathrm{~V}$ to $-10 \mathrm{~V}$. Furthermore, the study also showed that increased cathode flow rate led to a decrease in the plasma potential inside the ion beam about $10 \mathrm{~mm}$ downstream of the accel grid from about $50 \mathrm{~V}$ down to about $15 \mathrm{~V}$ relative to the beam collector.

In the wake of SERT II, ground testing continued on SERT-type mercury thrusters and cathodes. In 1982, Feng and Wilbur reported a series of experiments in which they studied the effect of magnetic fields on cathode coupling. ${ }^{8}$ In this work they show that 
magnetic fields as weak as the Earth's, about $50 \mu \mathrm{T}$ are capable of affecting the cathode coupling voltage. Furthermore, they show that stronger magnetic fields aligned axially with a hollow cathode affect emission processes and the formation of a sheath at the orifice, while those fields perpendicular to the cathode axis and parallel to the ion beam affect the coupling between the cathode and the ion beam. They suggest that the later process is caused by increased impedance between the beam and the cathode due to limited electron cross-field mobility. Finally, they show that direction of the fringing fields in the vicinity of the ion thruster, whether they curve toward the ion beam or away, can have a significant effect on the coupling.

\subsection{HET-Cathode Coupling}

Research focusing specifically on Hall thrusters and their cathodes has also been more prevalent in the past 10 to 15 years. Specifically, researchers have studied the affect of cathode type, cathode mass flow rate, and cathode position. Many of the trends that have been reported echo similar trends found in gridded ion thruster-cathode coupling.

\subsubsection{Cathode type}

Albaréde et al., have compiled the only study of which I am aware that specifically directly compares different types of cathodes operating with the same Hall-effect thruster. ${ }^{9}$ In this study, the authors compared one hot cathode and two self-heating cathodes. The first hot cathode, identified by the authors as being produced by the Moscow Technical Institute (MIREA), is very similar to the cathode used in these experiments, including the use of $\mathrm{LaB}_{6}$ emitter. The second cathode, manufactured by Laben S.p.A. Div. Proel Tecnologie had a 1-mm-diameter orifice, and the third, manufactured by the Kharkov Aviation Institute (KhAI) had a $0.4 \mathrm{~mm}$ diameter. Results from this study showed, not surprisingly, that while all three cathodes operated across a range of mass flow conditions, the MIREA had the worst cathode coupling. The Laben cathode performed slightly better than the KhAI cathode, however, the potential reasons for this are not discussed.

\subsubsection{Cathode mass flow rate}

It has repeatedly been noted that increased cathode flow rate improves cathode coupling voltage and performance in HETs. One of the earliest studies to show this was performed by Tilley, de Grys, and Myers at Primex Aerospace (now Aerojet). ${ }^{10}$ Tilley et al., studied the combination of a BPT-4000 HET and a prototype PAC hollow cathode. They noted that decreasing the cathode flow rate from $1.2 \mathrm{mg} / \mathrm{s}$ to $0.2 \mathrm{mg} / \mathrm{s}$ dropped $V_{\mathrm{cg}}$ 
from $\sim-10 \mathrm{~V}$ to $\sim-30 \mathrm{~V}$. In these experiments the thruster was operated at $350 \mathrm{~V}$, so this change represents a loss of about $6 \%$ of the available acceleration voltage for the ions. Over the same range of mass flows, they noted a $\sim 4 \%$ change in thrust.

In 2002, Manzella, Jankovsky and Hofer at NASA-Glenn Research Center reported on the development of a large, $50-\mathrm{kW}$ thruster. ${ }^{11}$ As part of this study, they again showed that cathode coupling voltage indeed decreases with decreasing cathode flow. As the flow in their cathode was lowered to from $7.5 \mathrm{mg} / \mathrm{s}$ to $2.5 \mathrm{mg} / \mathrm{s}$ the coupling voltages dropped from $\sim-10 \mathrm{~V}$ to between -20 and $-60 \mathrm{~V}$, depending on discharge current (which is directly related to anode mass flow). Here, reductions in anode efficiency of about $10 \% *$ were noted along with this change in cathode mass flow. Furthermore, the thruster could not be run at the higher voltages of $600 \mathrm{~V}$ and $700 \mathrm{~V}$ with the cathode operating at the lowest flow rate. Similar results were also seen by Manzella, Jacobson and Jankovsky operating on a smaller, $2.3 \mathrm{~kW}$ thruster. ${ }^{12}$

Albaréde et al., who studied the effect of cathode type mentioned in the previous section, also studied the effect of cathode flow rate. ${ }^{9}$ Using the 2-kW-class SPT-100 thruster, the authors again showed a decrease in coupling voltage with decreasing mass flow, regardless of the type of cathode used. For mass flow rates ranging from $0.7 \mathrm{mg} / \mathrm{s}$ down to $0.2 \mathrm{mg} / \mathrm{s}, V_{\text {cg }}$ dropped from about $-15 \mathrm{~V}$ down to about $-20 \mathrm{~V}$, while operating with a $300 \mathrm{~V}$ discharge voltage.

\subsubsection{Cathode position}

It has been repeatedly noted that cathode placement has an effect on thruster performance. An early study was reported by Jacobson and Jankovsky, ${ }^{13}$ which briefly notes that a cathode mounted at the "more traditional" angle of $45^{\circ}$ failed to initiate a discharge with the $200 \mathrm{~W}$ HET being tested. After reorienting the cathode to $90^{\circ}$ no problems were encountered.

The earliest report which focused only on HET-cathode coupling was performed by Tilley, et al., ${ }^{10}$ the same study previously mentioned in the Section 3.3.2. The authors of this study showed that thrust decreased about $2 \%$ when moving an axially aligned cathode from just outside the outer edge of the thruster body to a position $35 \mathrm{~mm}$ further away, radially (see Figure 2.6 to review cathode positioning terminology). However, when they angled the cathode at $45^{\circ}$, the trend reversed itself, showing a $<1 \%$ improvement over a similar range of radial positions. In both cases the cathode orifice was mounted $4.5 \mathrm{~mm}$ downstream of the exit plane. The authors of this study also moved the cathode axially, and noted a continuous improvement in $V_{\mathrm{cg}}$ as the cathode was moved from $-15 \mathrm{~mm}$ to $25 \mathrm{~mm}$. The authors claim that this was associated with a decrease in thrust, which is unexpected given the increase in $V_{\mathrm{cg}}$. However, they do not present the thrust data, and their analysis on this topic is brief.

${ }^{*}$ That is, a delta of $10 \%$, e.g. from $70 \%$ to $60 \%$. 
More recently, Walker and Gallimore studied the coupling over a much broader range. ${ }^{14}$ The primary focus of that research was understanding the behavior of cathodes in clusters of HETs, and that aspect of the work is beyond the scope of this document. However, as part of that study, Walker studied the effect of moving an axially aligned cathode from close to the outer edge of the thruster body to a radial distance of $110 \mathrm{~cm}$ from the closest approach point. Axially, the cathode was positioned approximately at the exit plane of the thruster. Their study used P5 5-kW-class HETs operating at 300 and $500 \mathrm{~V}$ and at two mass flow rates of $5.25 \mathrm{mg} / \mathrm{s}$ and $10.46 \mathrm{mg} / \mathrm{s}$. The authors report a change in $V_{\text {cg }}$ over this distance of between -4 and $-7 \mathrm{~V}$, depending on the exact operating conditions. At the same time, there was no discernable change in discharge current. Thrust, while showing some small changes on the order of $2 \%$ of the average value, did not show a consistent trend across all operating conditions. This is in contradiction to our own work in which we see generally improved performance with increasing cathode separation for cathodes mounted outside of the thruster body. ${ }^{15}, 16$ We will return to this briefly in Section 7.3.

Work led by Hofer showed that external magnetic fields influenced the coupling between the cathode and the HET. ${ }^{17,18,19,20}$ Hofer performed his work on a series of modified NASA-173M laboratory HET, a nominally 5-kW-class thruster. These thrusters had been modified to include magnetic trim coils which allowed the magnetic field topology of the thruster to be modified in situ. Hofer noted that, in particular, application of the external trim coil, may have improved the coupling voltage to the fixed-position cathode, while simultaneously raising the floating potential in the nearfield of the thruster. We will return to this briefly in Section 8.6.3.

Recent developments occurring at JPL have focused on mounting hollow cathodes in the center of Hall-effect thrusters, rather than outside. Internal mounting of the cathodes has been shown to decrease plume divergence, ${ }^{21}$ and increase overall efficiency of the thruster ${ }^{22}$ as compared to an axially-mounted, external cathode. In particular, the work authored by Jameson et al. shows improvements in several aspects of HET efficiency, including plume divergence, voltage utilization, current utilization, and cathode coupling voltage. ${ }^{22}$ Additionally, Jameson et al. show decreased plume potentials and increased cathode coupling voltages for internally versus externally mounted thrusters.

In addition to center-mount cathodes, cathode coupling has been investigated in alternative HET configurations including HET clusters, ${ }^{23,}{ }^{24}$ diverging cusped-field Hall thrusters, ${ }^{25}$ and cylindrical HETs. ${ }^{26,27}$ Of particular relevance to this work, a study by Raitses et al. using an annular configuration showed that as more current was drawn through the keeper electrode - a process which usually has the effect of heating the emitter-the potential near the exit plane of the HET was lowered, while the potential inside the thruster remained the same. This has the effect of causing more of the ion acceleration to occur inside the thruster, which can be expected to reduce beam divergence. In fact, this is exactly what was seen. ${ }^{27}$ 


\subsection{Summary}

A few facts stand out from this survey of research. First of all, it is clear that all researchers have considered $V_{\text {cg }}$ to be the standard measure of cathode coupling efficiency, with low, negative values indicating poor coupling leading to poor thruster efficiency, as theory suggests. Second, it has been repeatedly noted in both Hall-effect thrusters as well as gridded ion thrusters that decreasing cathode coupling voltages are almost always associated with elevated near-field plume potentials. Finally, it is well established that increasing the cathode mass flow rate improves the cathode coupling and the performance of Hall-effect thrusters. However, the total picture with regard to cathode mass flow is more complicated. At some point, increasing the cathode mass flow negatively impacts the system efficiency despite continued increases in the anode efficiency (see Section 2.2.2).

Despite these conclusions, many questions remain. It is certainly clear that cathode position affects the efficiency with which the thruster operates, though the precise way in which position affects the coupling is not understood. Furthermore, there is strong evidence to suggest that the external magnetic fields play an important role in determining this coupling behavior, but again, the precise manner in which it affects the coupling is unknown. Finally, the processes by which cathode coupling affects overall efficiency is not clear. The efficiency loss characterized by $V_{\mathrm{cg}}$ is part of the picture. But, other effects, such as beam divergence and voltage utilization, may also come into play.

It is likely that our lack of understanding is due to the paucity of studies explicitly dedicated to addressing the cathode coupling problem. Of all of the references mentioned in this chapter, only one ${ }^{9}$ of them has been explicitly designed to study cathode coupling on a single, standard configuration HET, ${ }^{*}$ and it did not include an investigation of cathode position. In fact, only a few have explicitly focused on HET-cathode coupling at all; ${ }^{14,22,24,27}$ the primary focus of the remainder has been on some other aspect of the thruster, and the cathode coupling was a footnote to the research. Clearly, a comprehensive study of HET-cathode coupling phenomena on a single, standard Halleffect thruster would be beneficial to the community.

\section{References for Chapter 3}

[1] Domonkos, M. T., Gallimore, A. D., and Patterson, M., "An Evaluation of Hollow Cathode Scaling to Very Low Power and Flow Rate," 25th International Electric Propulsion Conference, Cleveland, OH, August 24-28, 1997, Paper No. IEPC97-189.

${ }^{*}$ This statement is only strictly true if one considers a center-mounted cathode HET to be nonstandard. 
[2] Byers, D. C. and Snyder, A., "Parametric Investigation of Mercury Hollow Cathode Neutralizers," Journal of Spacecraft and Rockets, Vol. 8, No. 2, February 1971, pp. 133-139.

[3] Patterson, M. and Mohajeri, K., "Neutralizer Optimization," 22nd International Electric Propulsion Conference, October 14-17, 1991, Paper No. 91-151.

[4] Sovey, J. S., Rawlin, V. K., and Patterson, M., "Ion Propulsion Development Projects in U. S.: Space Electric Rocket Test 1 to Deep Space 1," Journal of Propulsion and Power, Vol. 17, No. 3, May 2001, pp. 517-526.

[5] Hall, D. F., Kemp, R. F., and Shelton, H., "Mercury Discharge Devices and Technology," AIAA Electric Propulsion and Plasmadynamics Conference, Colorado Springs, CO, September 11-14, 1967, Paper No. 67-669.

[6] Rawlin, V. K. and Pawlik, E. V., "A Mercury Plasma-Bridge Neutralizer," Journal of Spacecraft and Rockets, Vol. 5, No. 7, July 1968, pp. 814-820.

[7] Ward, J. W. and King, H. J., "Mercury Hollow Cathode Plasma Bridge Neutralizers," Journal of Spacecraft and Rockets, Vol. 5, No. 10, October 1968, pp. 11611164.

[8] Feng, Y.-C. and Wilbur, P. J., "The Influence of Stray Magnetic Fields on Ion Beam Neutralization," AIAA/JSASS/DGLR 16th International Electric Propulsion Conference, New Orleans, LA, November 17-19, 1982, Paper No. AIAA-821945.

[9] Albarède, L., Lago, V., Lasgorceix, P., Dudeck, M., Burgova, A., and Malik, K., "Interaction of a Hollow Cathode Stream with a Hall Thruster," 28th International Electric Propulsion Conference, Toulouse, France, March 17-21, 2003, Paper No. 03-333.

[10] Tilley, D. L., de Grys, K. H., and Myers, R. M., "Hall thruster-cathode coupling," 35th AIAA/ASME/SAE/ASEE Joint Propulsion Conference, June 20-24, 1999, Paper No. AIAA-99-2865.

[11] Manzella, D. H., Jankovsky, R. S., and Hofer, R. R., "A Laboratory Model 50kW Hall thruster," 38th AIAA/ASME/SAE/ASEE Joint Propulsion Conference, Indianapolis, IN, July 7-10, 2002, Paper No. AIAA-2002-3676.

[12] Manzella, D. H., Jacobson, D., and Jankovsky, R. S., "High Voltage SPT Performance," 37th AIAA/ASME/SAE/ASEE Joint Propulsion Conference, Vol. AIAA2001-3774, Salt Lake City, UT, July 8-11, 2001, Paper No. AIAA-2002-3676. (Also NASA TM-2001-21135.) 
[13] Jacobson, D. and Jankovsky, R. S., "Test Results of a 200W Class Hall Thruster," 34th AIAA/ASME/SAE/ASEE Joint Propulsion Conference, Cleveland, OH, July 13-15, 1998, Paper No. AIAA-98-3792. (Also NASA TM-1999209449.)

[14] Walker, M. L. R. and Gallimore, A. D., "Hall Thruster Cluster Operation with a Shared Cathode," Journal of Propulsion and Power, Vol. 23, No. 3, May 2007, pp. 528-536.

[15] Sommerville, J. D. and King, L. B., "Effect of Cathode Position on HallEffect Thruster Performance and Cathode Coupling Voltage," 43rd AIAA/ASME/SAE/ASEE Joint Propulsion Conference, Cincinnati, OH, July 8-11, 2007, Paper No. AIAA-98-3792.

[16] Sommerville, J. D. and King, L. B., "Effect of Cathode Position on Hall-Effect Thruster Performance and Cathode Coupling Voltage," 30th International Electric Propulsion Conference, Florence, Italy, September 17-20, 2007, Paper No. IEPC2007-78.

[17] Hofer, R. R. and Gallimore, A. D., "Recent Results From Internal and Very-NearField Plasma Diagnostics of a High Specific Impulse Hall Thruster," 28th International Electric Propulsion Conference, Toulouse, France, March 17-21, 2003, Paper No. IEPC-2003-037.

[18] Hofer, R. R. and Jankovsky, R. S., "Optimizing the Performance, Divergence, and Plasma Oscillations of High Specific Impulse Hall Thrusters," 28th International Electric Propulsion Conference, Toulouse, France, March 17-21, 2003, Paper No. IEPC-2003-142.

[19] Hofer, R. R., Jankovsky, R. S., and Gallimore, A. D., "High-Specific Impulse Hall Thrusters, Part 1: Influence of Current Density and Magnetic Field," Journal of Propulsion and Power, Vol. 22, No. 4, July 2006, pp. 721-731.

[20] Hofer, R. R., Development and Characterization of High-Efficiency, High-Specific Impulse Xenon Hall Thrusters, Ph.D. dissertation, University of Michigan, Ann Arbor, MI, 2003.

[21] Hofer, R. R., Johnson, L. K., Goebel, D. M., and Fitzgerald, D. J., "Effects of an Internally-Mounted Cathode on Hall Thruster Plume Properties," 42nd AIAA/ASME/SAE/ASEE Joint Propulsion Conference, July 9-12, 2006, Paper No. AIAA2006-4482. 
[22] Jameson, K. K., Goebel, D. M., Hofer, R. R., and Watkins, R. M., "Cathode Coupling in Hall Thrusters," 30th International Electric Propulsion Conference, Florence, Italy, September 17-20, 2007, Paper No. IEPC-2007-278.

[23] Hargus, W. A. and Reed, G., "The Air Force Clustered Hall Thruster Program," 38th AIAA/ASME/SAE/ASEE Joint Propulsion Conference, July 7-10, 2002, AIAA-2002-3678.

[24] Beal, B. E., Gallimore, A. D., and Hargus, W. A., "The Effects of Cathode Configuration on Hall Thruster Cluster Plume Properties," 41st AIAA/ASME/SAE/ASEE Joint Propulsion Conference, July 10-13, 2005, Paper No. AIAA-2005-3678.

[25] Courtney, D. G. and Martìnez-Sànchez, M., "Diverging Cusped-Field Hall Thruster (DHCT)," 30th International Electric Propulsion Conference, Florence, Italy, September 17-20, 2007, Paper No. IEPC-2007-39.

[26] Raitses, Y., Smirnov, A. A., and Fisch, N. J., "Enhanced performance of cylindrical Hall thrusters," Applied Physics Letters, Vol. 90, May 2007, Paper No. 221502.

[27] Raitses, Y., Granstedt, E., Smirnov, A. A., Merino, E., and Fisch, N. J., "Effects of Cathode Electron Emission on Hall Thruster Discharge," 44th AIAA/ASME/SAE/ASEE Joint Propulsion Conference and Exhibit, Hartford, CT, July 2123, 2008, Paper No. AIAA-2008-5188. 


\section{Chapter 4}

\section{Experiments}

\subsection{Overview}

The experiments presented in this work were designed to improve our understanding of the effect of cathode position on HET-cathode coupling and thruster performance. The goal was to gain insight into the cathode coupling mechanism, which should support efforts to optimize the configuration and operation of HETs.

I conducted three experiments. Experiment 1, the "Preliminary Experiment," was designed to study how cathode position affects thruster efficiency. It explored a physically large area of cathode positions across a range of magnetic field strengths and compared the performance at each condition. These results were also compared to a magnetic field model of the thruster. This experiment lead to the hypothesis of the importance of the magnetic field separatrix. Experiment 2, the "Separatrix Crossing Experiment," studied a more limited range of cathode positions in an effort to confirm the hypothesis. In addition to thruster performance measurements, this experiment also incorporated detailed studies of the ion beam properties and near-field plume plasma properties. Experiment 3, title "Extended Outer Pole Performance Experiment," provides additional confirmation of the hypothesis, measuring performance versus separatrix location for a range of magnetic fields strengths.

This chapter outlines the setup of the experiments. Following this chapter, Chapter 5 discusses the electrostatic probes used in these experiments, while Chapter 6 discusses the magnetic field models. These chapters are followed by Chapters 7 through 9 which discuss each of the specific experiments and present the results. Not all aspects of the general experiment described here were used in each of the individual experiments. As the Separatrix Crossing Experiment was the most complex of the experiments, it will serve as the basis for this overview. The equipment and procedures of the simpler experiments are the same as in the Separatrix Crossing Experiment, unless otherwise noted. 


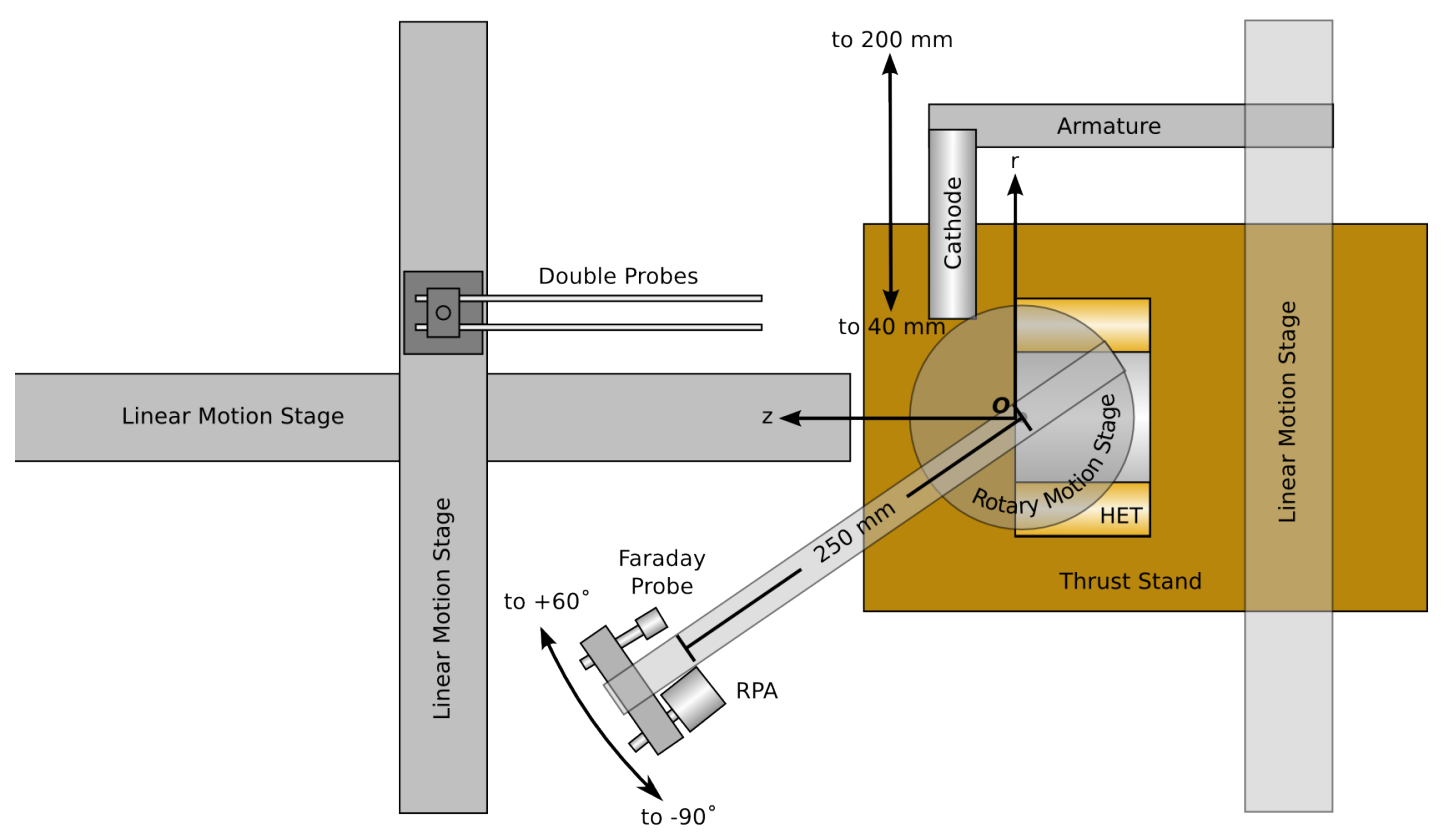

Figure 4.1: Experimental setup

\subsection{Setup}

Figure 4.1 shows the typical experimental setup. In each of the experiments, thrust was measured with an inverted-pendulum thrust stand on which the HET was mounted. From thrust and additional telemetry such as discharge current, discharge voltage and mass flow rates, the efficiency was calculated. In the Preliminary and Separatrix Crossing experiments, the cathode was mounted on a repositionable boom. In the Separatrix Crossing Experiment the ion beam and plume properties were measured using electrostatic probes. Specifically, a Faraday probe measured the ion beam current density, and a retarding potential analyzer (RPA) measured ion energy distribution functions. A double Langmuir probe was mounted on a two-axis motion table and measured nearfield plume plasma properties. A second double probe was typically mounted and used as a backup.

Figure 4.3 shows the electrical setup of the HET and cathode. Note that the cathode is isolated from ground when the keeper power supply is disconnected, and the discharge voltage is applied relative to the cathode. The keeper power supply used was improperly isolated from ground. Thus, it was necessary to not only disconnect the keeper electrode to allow the keeper to float, it was also necessary to disconnect the keeper power supply from the cathode to allow the HET system to float. As these ex- 


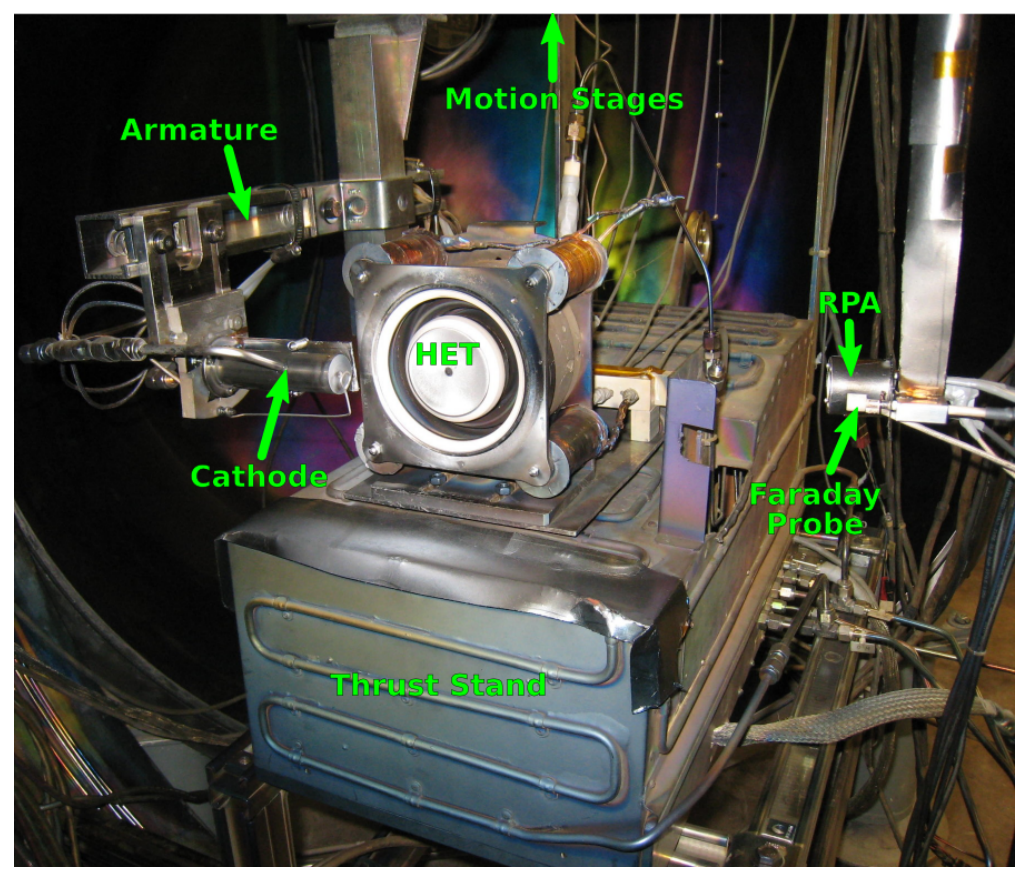

Figure 4.2: Photograph of experimental setup

periments were run without the keeper, this presented no problem, but merely added the extra step of disconnecting the power supply to the procedure listed below.

\subsection{Procedure}

The following procedure was general to all of experiments conducted in this work:

1. Mount thruster, cathode and probes

2. Ensure thrust axis is aligned parallel to the $\mathrm{z}$-axis motion table* Alignment was initially done by sight. Later, a laser level was incorporated into this process.

3. Position cathode at a nominal starting location and measure the offset between the cathode and the thruster origin ${ }^{\dagger}$

4. Align probes with the thruster origin, if applicable

5. Calibrate thrust stand to ensure proper functioning

*This axis moved either the cathode or the double probes, depending on the experiment.

${ }^{\dagger}$ The thruster origin is defined as the intersection of the thruster centerline with the exit plane. 


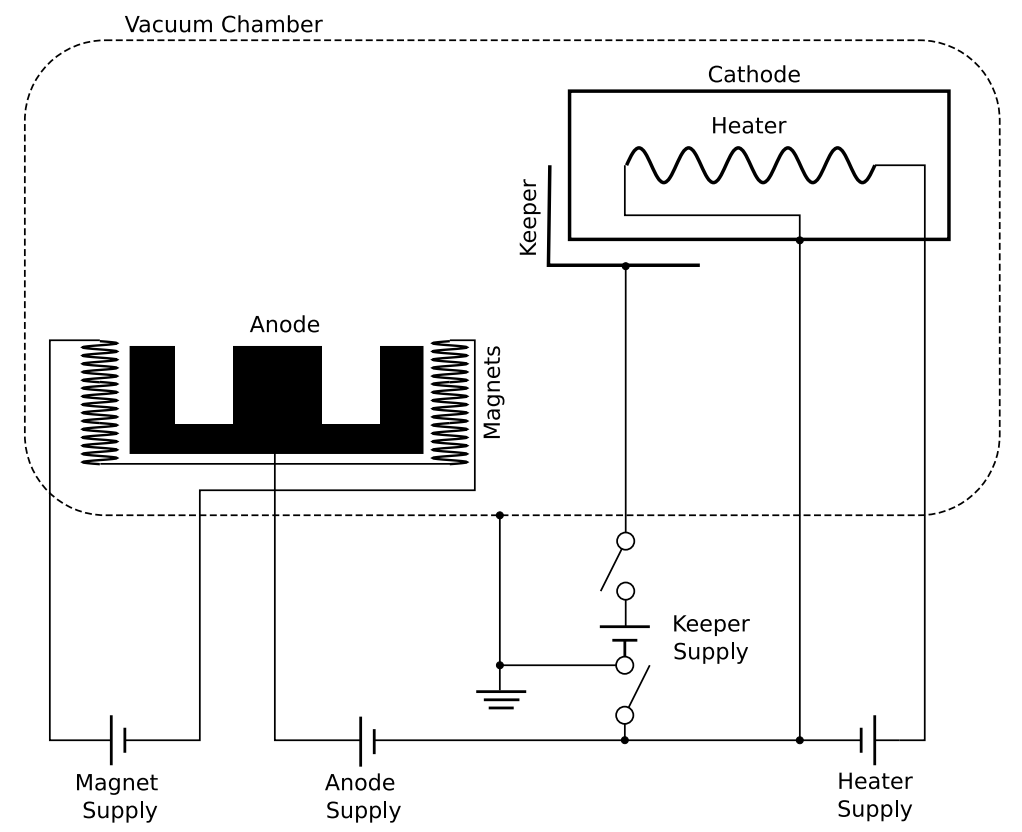

Figure 4.3: Electrical schematic of the HET and cathode

6. Close tank and pump down

7. Recalibrate thrust stand to ensure proper functioning

8. Condition and light cathode (see Section 4.4.4)

9. Set the HET magnets to the desired current

10. Flow gas through the thruster at 4 or $5 \mathrm{mg} / \mathrm{s}$ Xe or equivalent volumetric flow rate of $\mathrm{Kr}$

11. Apply $250-300 \mathrm{~V}$ between the cathode and the main anode

12. If necessary, reduce the magnet current until the thruster lights

13. Un-power and disconnect keeper electrode and power supply

14. Reset HET magnets to the desired current or find optimal operation point by adjusting magnet current while monitoring efficiency

15. Allow thruster to warm up for at least 1 hour 
16. Shut off main anode power and gas flow

17. Recalibrate thrust stand. This calibration will be used. This step typically took about ten minutes.

18. Repeat steps 9 to 14

19. Acquire data (exact procedure described in Chapters 7, 8 and 9)

20. Shut off main anode power and gas flow

21. Shut off cathode heater

22. Flow cathode gas for at least 10 minute to cool the cathode

23. Stop cathode gas

24. Stop pumps

\subsection{Equipment}

\subsubsection{Diagnostics}

The thrust stand was a NASA-Glenn style, null displacement, inverted pendulum thrust stand, with automatic leveling. The displacement nulling was provided by a solenoid driven by a closed-loop digital controller which measured the displacement of the thrust stand using a linear voltage displacement transducer (LVDT). The level was sensed by an electrolytic inclinometer, and controlled by a microstepping motor connected to an 80-threads-per-inch screw. The thrust is directly proportional to the current provided to the solenoid. Calibration was provided by a linear fit to a set of known weights applied to the thruster across a pulley and controlled via a stepper motor. Typically the calibration was run immediately before the thruster was powered on and then again after the thruster had been warmed up.

The data from the thrust stand and the power supplies was continually monitored by a telemetry datalogger implemented in software. Communication with the thrust stand and the power supplies was either by analog input to the datalogging computer, or via GPIB, depending on the device. Telemetry was recorded at a rate of $1 \mathrm{~Hz}$. From the recorded thrust, anode mass flow rate, discharge current and discharge voltage, the measured efficiency is calculated according to Equation 2.20. Typically, both anode efficiency (Equation 2.22) and total efficiency (Equation 2.23) are calculated.

Some of these experiments also utilized a Faraday probe, a retarding potential analyzer, and double Langmuir probes. Each of these will be discussed in detail in Chapter 5 . 


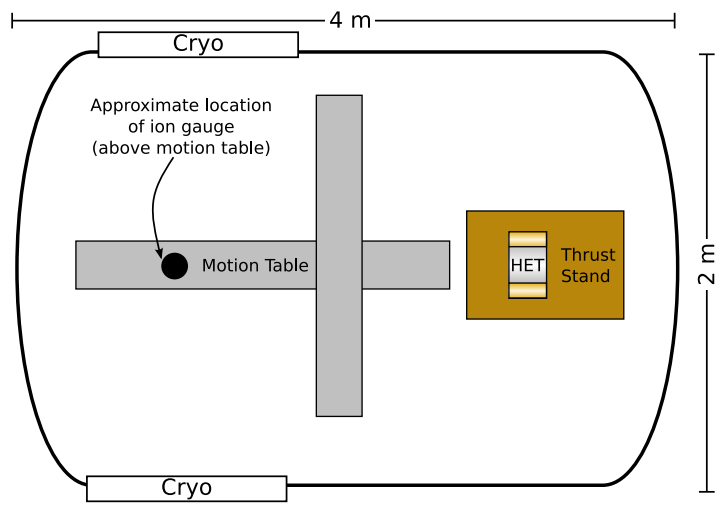

Figure 4.4: Schematic of the vacuum facility showing locations of key components

\subsubsection{Vacuum facility}

All experiments were run in the Xenon Vacuum Facility at Michigan Tech's Ion Space Propulsion Laboratory. The facility is a 4-m-long chamber $2 \mathrm{~m}$ in diameter. It is evacuated by two 48 -inch cryogenic pumps capable of $60,000 \mathrm{~L} / \mathrm{s}$ of nitrogen each. The base pressure during these experiments was $5 \times 10^{-6}$ Torr and operating pressures did not exceed $2.4 \times 10^{-5}$ Torr as measured by an ion gauge corrected for xenon and located on the top of the downstream third of the chamber (see Figure 4.4).

\subsubsection{Hall thruster}

The HET used in this experiment was an Aerojet BPT-2000, 2-kW-class thruster. ${ }^{1}$ The thruster has an outer diameter of $\sim 100 \mathrm{~mm}$ and a channel width of $\sim 10 \mathrm{~mm}$. It operates at a nominal voltage of $300 \mathrm{~V}$ and a mass flow of $5 \mathrm{mg} / \mathrm{s}$ xenon. At these conditions its specific impulse is $\sim 1700$ s with $\sim 50 \%$ efficiency.

\subsubsection{Cathode}

The cathode used in these experiments was a laboratory cathode similar to the MIREA cathode used by Albadére. ${ }^{2}$ It is shown schematically in Figure 4.5. The cathode consists of a 1-inch-diameter, titanium cylinder approximately $100 \mathrm{~mm}$ long. A 2-mm diameter orifice was drilled in one face. Pressed against this hole is a molybdenum pellet holder which holds a lanthanum-hexaboride $\left(\mathrm{LaB}_{6}\right)$ emitter. Xenon is introduced into the cathode via a feed tube attached to the side of the cylinder. Filling the length of the cathode from the pellet holder to the base is a tungsten heater coil which heats the emitter to its operating temperature. Radiation insulation loosely 
wraps the heater and pellet holder to maximize emitter heating. A keeper electrode is positioned approximately $3 \mathrm{~mm}$ outside of the orifice and is used to ignite the cathode discharge. After the main HET discharge was ignited, the keeper was unpowered and allowed to float.

The conditioning procedure for the $\mathrm{LaB}_{6}$ cathode is simple.

1. Flow gas through the cathode at 10 SCCM for 5 minutes.

2. Apply $6 \mathrm{~A}$ of current to the heater for 10 minutes. This corresponded to approximately $60 \mathrm{~W}$ of power.*

3. Apply $10 \mathrm{~A}$ of current to the heater for 10 minutes. This corresponded to approximately $200 \mathrm{~W}$.

4. Apply $300 \mathrm{~V}$ to the keeper electrode with a $0.5 \mathrm{~A}$ current limit. Discharge should begin immediately. Typical voltages after discharge ignition were about $50 \mathrm{~V}$.

5. If discharge does not begin, slowly increase the heater current in $0.5 \mathrm{~A}$ increments until it does.

6. Reduce cathode heat to $6 \mathrm{~A}$.

In these experiments, the cathode was placed near and within the ion beam. In early trials it was found, not surprisingly, that this caused an increase in cathode temperature. While the temperature of the cathode was not directly monitored, the heater voltage was seen to increase while it was held at constant current, a sure indication of a temperature increase. Any heating of the cathode causes the electron emission current from the emitter to increase, which, in turn, affects the cathode coupling voltage. While this is interesting and may bear further research, in these experiments, we wanted to avoid this affect and focus on the effect of where the cathode gas and electrons are introduced.

To alleviate the thermal loading of the cathode, we constructed an actively cooled shield. The shield, pictured in Figure 4.5c, is a copper fin which is slightly wider than the cathode diameter and extends the length of the cathode. One-quarter-inch outer-diameter copper tubing is soldered to the base of the fin, through which water flows during operation. The exposed portion of the fin is covered in graphite foil to prevent copper from contaminating the thruster or other equipment. Tests with the heat shield showed significant reduction in cathode heating. Without the shield, moving the cathode from outside the beam at $120 \mathrm{~mm}$ to inside the beam at $60 \mathrm{~mm}$ resulted in a change of $0.6 \mathrm{~V}$ on a nominally $10.5 \mathrm{~V}$ heater voltage. With the shield in place, the

${ }^{*}$ The cathode heater was rebuilt several times throughout the experiments. Each time the length of the heater was maintained. None-the-less, there was approximately a $10 \%$ variability in heater power for a given current. Future experiments should strive to maintain constant heater power, rather than constant current. 


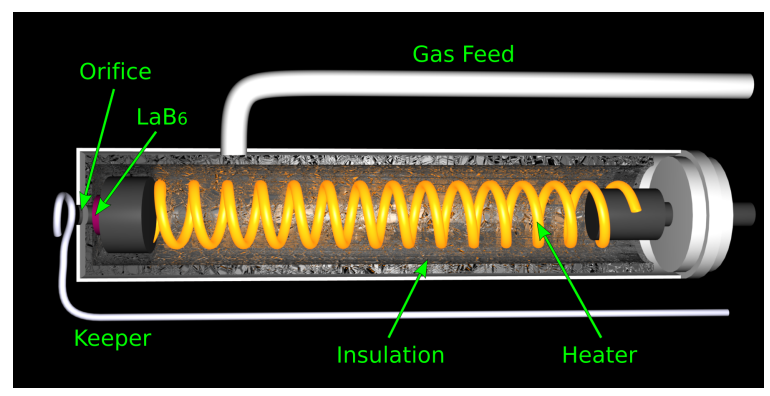

(a)

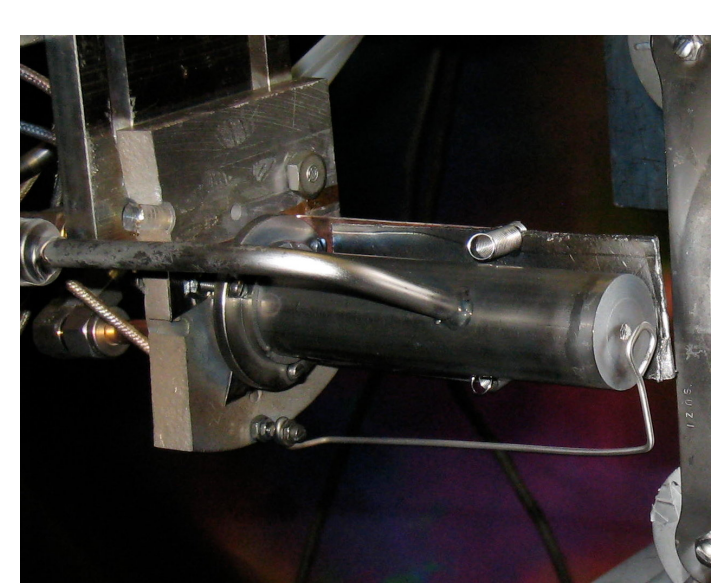

(b)

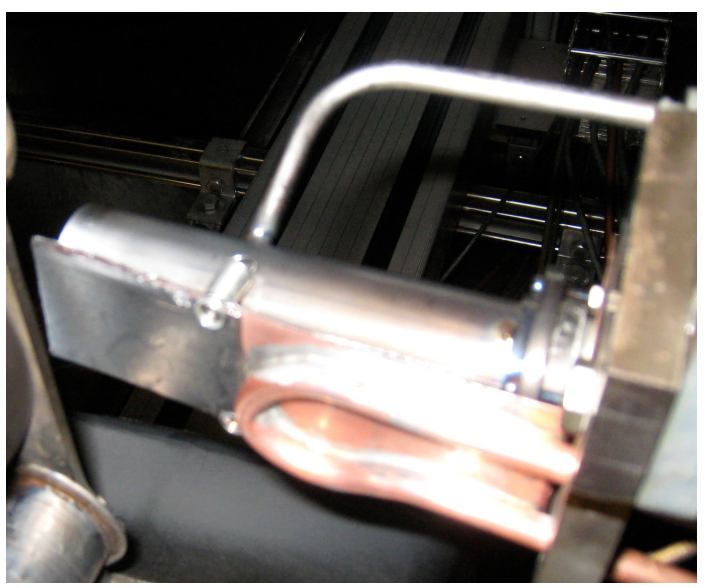

(c)

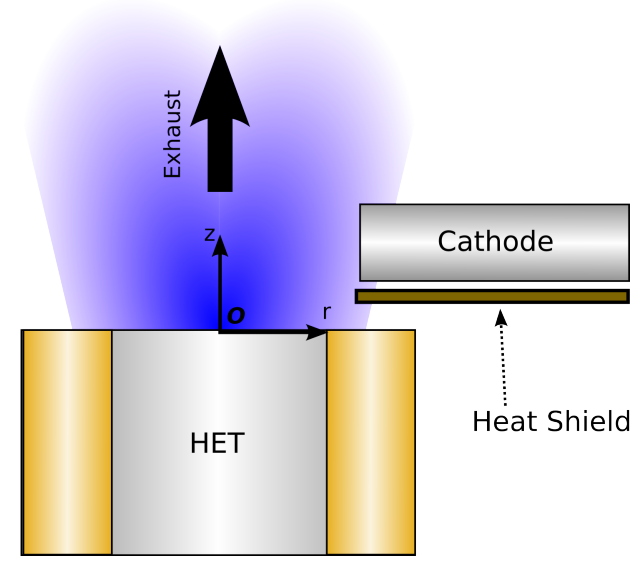

(d)

Figure 4.5: (a) Cut-away view of the laboratory cathode (b) Photograph (c) The heat shield (d) The heat shield blocks the beam, alleviating beam-induced cathode heating. 
same change in position caused a $0.1 \mathrm{~V}$ change in heater voltage. Heater currents were $6 \mathrm{~A}$ in both cases.

\subsubsection{Mass flow controllers}

Flow of xenon to the thruster and the cathode was controlled by MKS Type 1479a mass flow controllers. Their accuracy was tested by monitoring the rise in pressure of a small calibration tank of known volume while flowing gas into the tank. The mass flow controllers are accurate to $1 \%$ of the full scale of the controller. The full scale for the anode controller was $200 \mathrm{SCCM}(19.5 \mathrm{mg} / \mathrm{s}$ xenon), and it was $20 \mathrm{SCCM}(1.95 \mathrm{mg} / \mathrm{s}$ xenon) for the cathode controller.

\section{References for Chapter 4}

[1] King, D., Tilley, D. L., Aadland, R., Nottingham, K., Smith, R., Roberts, C., Hruby, V., Pote, B., and Monheiser, J., "Development of the BPT Family of U.S.Designed Hall Current Thrusters for Commercial LEO and GEO Applications," 34th AIAA/ASME/SAE/ASEE Joint Propulsion Conference, July 13-15, 1998, Paper No. AIAA-98-3338.

[2] Albarède, L., Lago, V., Lasgorceix, P., Dudeck, M., Burgova, A., and Malik, K., "Interaction of a Hollow Cathode Stream with a Hall Thruster," 28th International Electric Propulsion Conference, Toulouse, France, March 17-21, 2003, Paper No. 03-333. 


\section{Chapter 5}

\section{Electrostatic Probes}

\subsection{Langmuir Probe}

The probes used in the present experimental work were all derived from the electrostatic plasma, or "Langmuir", probe. It was first described by Langmuir and Mott-Scott in $1924 .{ }^{1}$ It is simply a piece of wire or other electrode placed within the plasma in the region to be characterized. A power supply drives the potential on the probe, causing it to sweep from below plasma potential to above it. The current collected by the probe is recorded and the resulting current vs. voltage (I-V) curve is plotted, as shown in Figure 5.1. From this trace one may determine the plasma potential, electron density and electron temperature.

When the probe is biased strongly negative relative to the plasma potential, it repels the nearby electrons* while attracting the nearby ions, recording a negative current (current into the probe). This is the ion saturation region of the I-V trace. As the probe voltage increases, the highest energy electrons may overcome the bias and hit the collector, while the highest energy ions may not be sufficiently attracted to the probe to collide with it. Thus, a higher current is recorded. This begins the exponential growth region. At zero current, the probe is at floating potential, by definition. As the bias is swept between floating potential and plasma potential fewer and fewer electrons are repelled. As the potential is raised above plasma potential, the electrons are attracted to the probe. At this point, an ideal probe reaches electron saturation, where all of the electrons within the sheath are collected, all the ions are repelled, and no more current will be collected, irrespective of the applied potential.

In real probes a "sheath" forms around the probe. As the potential of the probe is increased the disturbance to quasi-neutrality caused by the probe will extend further into the plasma as the free charges within the plasma are decreasingly able to move to counteract the disturbance caused by the probe. This causes an increased volume of

${ }^{*}$ That is, the electrons within the sheath. We will address the sheath concept momentarily. 
potential higher than plasma potential—not only on the volume of the probe itself, but also in the plasma surrounding the probe. In reality, this volume is infinite, but with decreasing potential as distance from the probe increases. However, it is conceptually useful to think of it as a sheath of finite extent, as shown in Figure 5.2. Now, in addition to the surface area of the probe, the surface area of the sheath also serves to attract or repel electrons, depending on the sign of the potential. Thus, as the potential of the probe increases, the effective collection area of the probe also increases. Because of this, real probes never achieve total saturation. Instead, a "knee" normally appears in the I-V curve, which indicates the transition to "saturation."

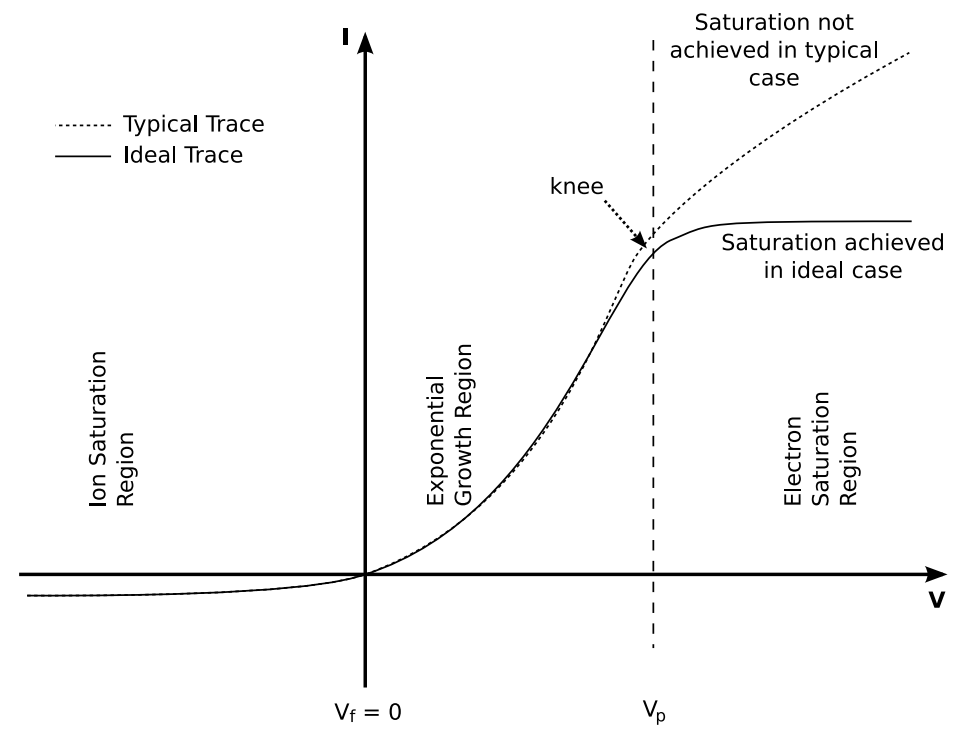

Figure 5.1: Typical and ideal Langmuir probe traces, showing the various regions of the trace

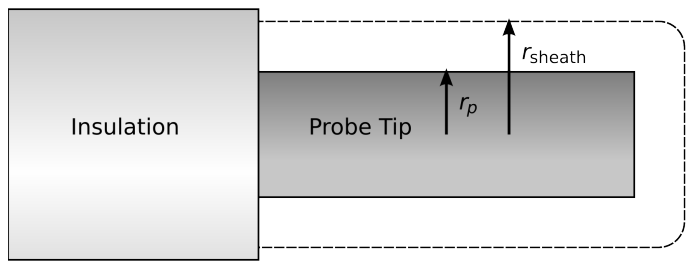

Figure 5.2: A sheath forms around Langmuir probes at high potentials 
Langmuir probes come in a variety of shapes, the most common being planar, cylindrical, and spherical. Due to their high symmetry, these shapes are most easily dealt with in theory. Planar probes are the least susceptible to sheath expansion, however, cylindrical probes are the simplest to construct - the exposed tip of a wire sufficesand that is the geometry used in these experiments.

The sheath expansion gives rise to the concept of regimes of probe operation. If a probe is operated in a plasma such that the change in sheath does not substantially affect the effective area of the probe, then the probe is said to be in the "thin-sheath limit" (TSL). At the other end of the spectrum, at least for cylindrical and spherical probes, the sheath may grow so large compared to the probe that the current collected is no longer a function of the actual probe area, but rather of the "orbital mechanics" of a particle entering the sheath area. This is the so-called "orbit motion limit" (OML). The two regions to not overlap but are separated by the "transitional regime."

To determine in which regime a probe is operating one compares the Debye length, given by

$$
\lambda_{d}=\sqrt{\frac{\varepsilon_{0} k T_{e}}{e^{2} n_{e}}}
$$

to the radius of the probe, $r_{p}$. The ratio of probe radius to Debye length is the nondimensional probe radius

$$
\xi=\frac{r_{p}}{\lambda_{d}} .
$$

When $\xi \gg 1$ the probe is operating in the TSL. When $\xi \ll 1$ the probe is in the OML.

Two problems immediately present themselves. The first is that it is necessary to know $T_{e}$ and $n_{e}$ in order to determine in which regime the probe is operating. However, $T_{e}$ and $n_{e}$ are the very quantities that we are attempting to measure. This problem is usually avoided by simple estimation. Based on prior measurements of similar HETs, I expected $1 \mathrm{eV}<T_{e}<12 \mathrm{eV}$ and $10^{15} \mathrm{~m}^{-3}<n_{e}<10^{18} \mathrm{~m}^{-3}$. This yields Debye lengths in the range $250 \mu \mathrm{m}>\lambda_{d}>25 \mu \mathrm{m}$.

The second problem is that when using a probe over a wide range of plasma conditions the probe may not always be in the same regime. One can avoid this by making the probe either very big or very small in comparison to the Debye length. However, there are problems with both. It is difficult to make a small probe. Furthermore, a small probe is more susceptible to heating, ablation, and destruction in the harsh plasma environment in which it must operate. On the other hand, a big probe limits the spatial resolution that can be achieved, and furthermore, may draw a significant amount of current, thereby disturbing the measured plasma. To avoid these problems, Peterson and Talbot developed an analytical approach which uses adjustable parameters to find the best fit to the probe trace, regardless of the operating regime. ${ }^{2}$ This method will be further discussed in Section 5.4 


\subsection{Faraday Probe}

\subsubsection{Theory}

The Faraday probe is simply a Langmuir probe biased at a fixed, negative voltage such that it repels all electrons and collects all ions. Typically these probes are planar, and are designed to collect the ion beam current. By passing the probe through the ion beam, the ion current density can be determined by dividing the current to the probe by the exposed probe area

$$
J=I_{\text {probe }} / A_{\text {probe }} \text {. }
$$

Typically the probe is swept through an arc at a fixed distance from the center of the probe. In this fashion, the divergence of the ion beam can be assessed.

If a simple probe is used, the plasma sheath around the probe can have the effect of artificially increasing the collecting area of the probe by an unknown amount, as shown in Figure 5.3a. To alleviate this problem, Faraday probes are often designed with a guard ring, a second collector positioned coaxially to the primary collector. If both the probe and the guard electrodes are biased to the same potential and the guard ring is separated from the probe by a distance less than the Debye length, the sheath in front of the probe electrode will be parallel to the probe surface (Figure 5.3b), and, thus, the effective collection area will match the area of the probe. Of course, the sheath effects are still present, but this now affects only the current to the guard, which is not measured.

\subsubsection{Bias electronics}

To bias both guard and probe, one may use two independent power supplies, set to the same voltage. In order to simplify the process, however, I designed a circuit which would maintain an applied voltage from a single power supply on both rings, while allowing the current to the probe electrode to be measured independently from the current to the guard. The circuit, shown in Figure 5.4, measures the current to the probe electrode by amplifying the voltage drop across a precision $10 \Omega$ shunt resistor with a gain of $\sim 10.1$, as determined by $\mathrm{R} 2$ and $\mathrm{R} 3$. This potential (relative to Vbias) is then measured at Vout. To maintain the guard at the same potential, a FET, operating as a voltage-controlled resistor, is placed inline with the guard electrode. A precision op amp compares the voltage between the two electrodes, and adjusts the resistance of the FET to ensure that the voltages of the electrodes are the same, regardless of differing currents flowing through the two electodes. The capacitor $\mathrm{C} 1$ prevents high frequency oscillations. The entire circuit is biased to repel electrons and collect ions by a single, external power supply, connected to Vbias. The op amps are powered by 6 AA batteries, which float with the applied bias. 


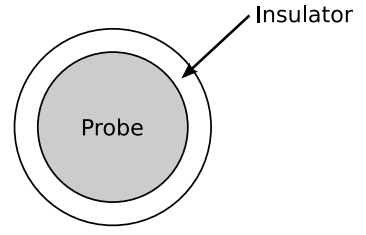

Probe face

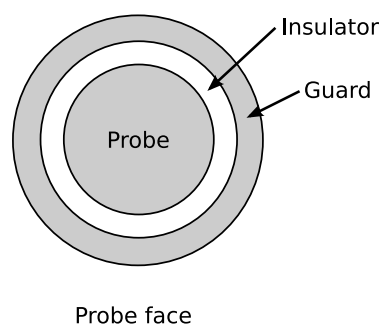

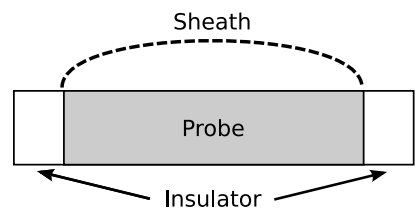

Cross-section

(a) Unguarded

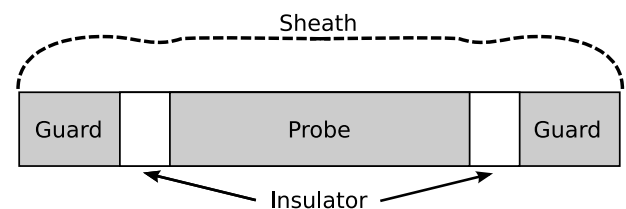

Cross-section

(b) Guarded

Figure 5.3: Sheaths of a unguarded and guarded Faraday probe

The linearity of the current shunt amplifier was checked using a milliamp power supply to source current into Vprobe. Vout was monitored with a hand-held multimeter. The results are plotted in Figure 5.5. The inverse of the resulting linear regression was used to convert the voltage readout from the circuit into probe current values.

\subsubsection{Design and operation}

The Faraday probe used in these experiments was made from a tungsten rod $2.4 \mathrm{~mm}$ in diameter. The guard is separated from the probe by an alumina tube with an outer diameter of $4.75 \mathrm{~mm}$. The guard ring, made of stainless steel, surrounds the alumina. The outer diameter of the guard ring is $10 \mathrm{~mm}$. Except for the face, the guard ring is spray coated with boron-nitride to reduce the amount of current collected by the ring.

The probe was mounted on a boom capable of swinging through the off-thrust-axis angle. The probe was mounted at a distance of $250 \mathrm{~mm}$ and swept from an angle of -90 degrees to +60 degrees (see Figure 4.1) at 2.3 degree intervals. Beyond 60 degrees the probe would collide with the cathode. At each interval, the voltage from the shunt resistor amplifier was measured by a computerized data acquisition system, and converted into a current. At each angle 1000 measurements were made at a rate of $10,000 \mathrm{~Hz}$, and the average of these measurements was used. 


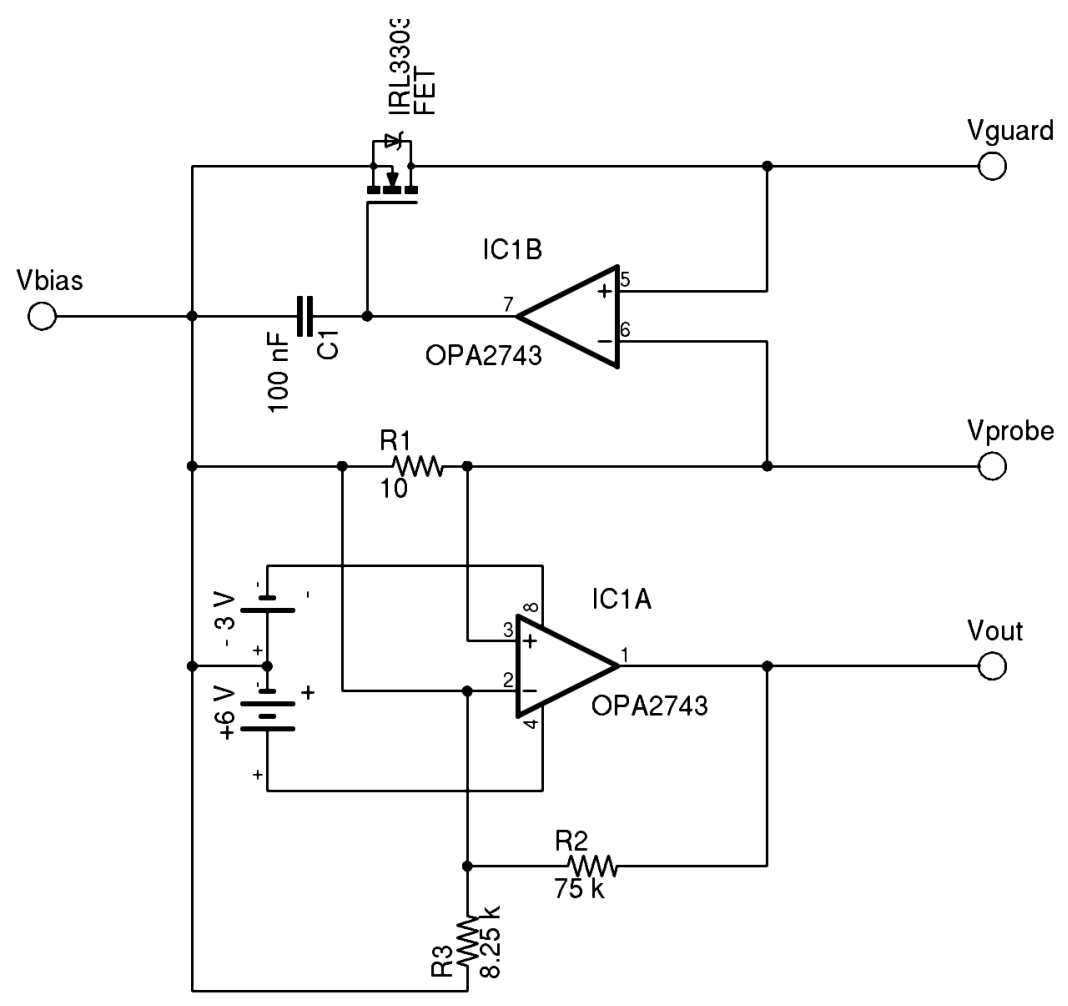

Figure 5.4: Schematic for the Faraday probe driver circuit

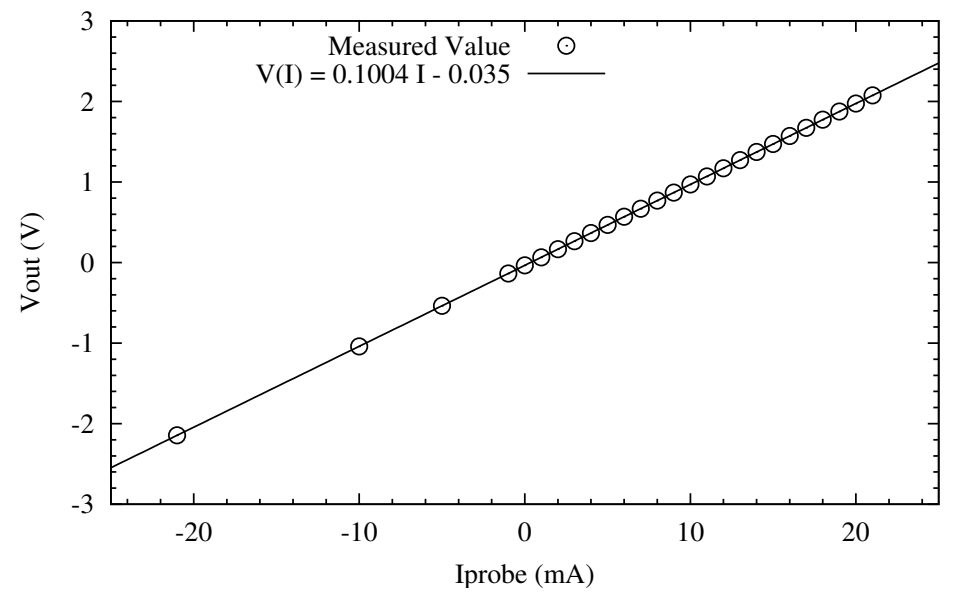

Figure 5.5: Experimental measurement of gain for the Faraday probe driver circuit 


\subsubsection{Sources of error}

\section{Secondary electron emission}

Ions impacting the surface of the collector will cause secondary electrons to be emitted from the surface of the electrode through Auger ejection. Because of the negative bias of the electrode with respect to plasma potential, once ejected, these electrons are unlikely to return to the electrode. Thus, an additional current above that of the beam ions is recorded. This effect is typically small. For clean tungsten the expected rates are $0.02 \mathrm{e}^{-}$/ion for $\mathrm{Xe}^{+}$and $0.2 \mathrm{e}^{-}$/ion for $\mathrm{Xe}^{2+} .3$ For krypton the values are slightly higher, $0.05 \mathrm{e}^{-}$/ion for $\mathrm{Kr}^{+}$and $0.3 \mathrm{e}^{-}$/ion for $\mathrm{Kr}^{2+}{ }^{3}$ Assuming an ion beam comprised of $90 \%$ singly-charged ions and $10 \%$ doubly-charged ions, this yields an average secondary electron emission rate of $0.038 \mathrm{e}^{-}$/ion for xenon and $0.075 \mathrm{e}^{-}$/ion for krypton. Therefore, we can expect the ion current densities of the Faraday probe to overstate the true densities by roughly $5 \%-10 \%$, due to this effect.

\section{Facility effects}

The presence of background neutrals in the vacuum facility can lead to two more sources of errors in measuring ion current with Faraday probes, which has been the focus of significant research. ${ }^{4,5,6}$ The root of the problem is charge-exchange (CEX) collisions, which result in two effects. First, the beam ions are attenuated due to the collisions. An ion beam with current density $j$ is attenuated to a value of $j_{z}$ after traveling a distance $z$ according to ${ }^{4}$

$$
j_{z}=j \exp \left(-n_{0} \sigma_{c e} z\right)
$$

For $210 \mathrm{eV}$ ions, the cross-section for xenon is approximately $50 \AA^{2}$ while for krypton it is closer $35 \AA^{2}{ }^{7}$ For background pressures of $\sim 1^{-5}$ Torr, assuming a background neutral temperature of $298 \mathrm{~K}$, and a path length of $250 \mathrm{~mm}$ this yields an attenuation, $j_{z} / j$ of 0.96 for xenon and 0.97 for krypton. This effect causes the probe to underestimate the current by 3-4 percent.

However, a second effect which is attributed to charge exchange causes the probe to significantly overestimate the current density. In addition to the main beam ions collected by the Faraday probe, the slow moving CEX ions are also collected. ${ }^{4,5,6,8}$ The current from the CEX ions contributes a significant amount to the measured current density. In fact, Rovey et al. measured integrated beam currents which were $35 \%$ lower when using a probe that filtered out the slower moving and large-impact-angle CEX ions. ${ }^{4}$ The effect on measurements of beam divergence, however, was less, showing a 3-6 degree (10 - 20\%) change in $90 \%$ plume divergence half-angle. 


\section{Sheath effects}

Due to errors in the original design of the Faraday probe, the gap between the probe and the guard is larger than the expected Debye length in the plasma where the probe operates. Therefore, the guard ring is not effective, and sheath expansion effects are still present (see Section 5.2.1). In this region of the plume, electron temperatures on the order of $3 \mathrm{eV}$ are expected, while the electron density is expected to be no less than $10^{17} \mathrm{~m}^{-3}$. For these conditions, the Debye length is about $40 \mu \mathrm{m}$. However, as this size is negligible compared to the $2.4 \mathrm{~mm}$ diameter of the probe, the sheath effects can be disregarded.

\subsection{Retarding Potential Analyzer}

A retarding potential analyzer (RPA) is an electrostatic device for measuring the energy distribution function (EDF) of the incident charged particles. The basic design consists of a metal grid with an applied voltage that may be swept to deflect (retard) particles of "low" energies and prevent them from reaching the detector placed behind it." Figure 5.6 depicts the RPA used in these experiments. For an RPA designed to measure the ion EDF, the retarding grid is swept from the reference potential to more positive potentials until no current is detected on the plate. At the reference potential, all ions are collected by the detector, and as the retarding potential is raised the lower energy ions are deflected away from the detector. The EDF, $f(E)$, is therefore proportional to

$$
f(E) \propto-\frac{1}{q} \frac{d}{d V} I(E)
$$

The exact EDF may be determined from this equation by normalizing its area such that:

$$
\int_{0}^{\infty} f(E) d E=1 .
$$

Since the energy of an incident particle is directly related to the voltage on the detector by $E=q e V$ we can change the independent variable in Equation 5.5 from $\mathrm{E}$ to $\mathrm{V}$, where $\mathrm{V}$ is the voltage of the detector plate-the parameter under experimental control.

As with Faraday probe, secondary electrons ejected from the collector can cause an additional current to be recorded. The secondary electrons may be suppressed through the addition of a repelling grid, which prevents secondary electrons from escaping. Instead, they return to the collector plate. 


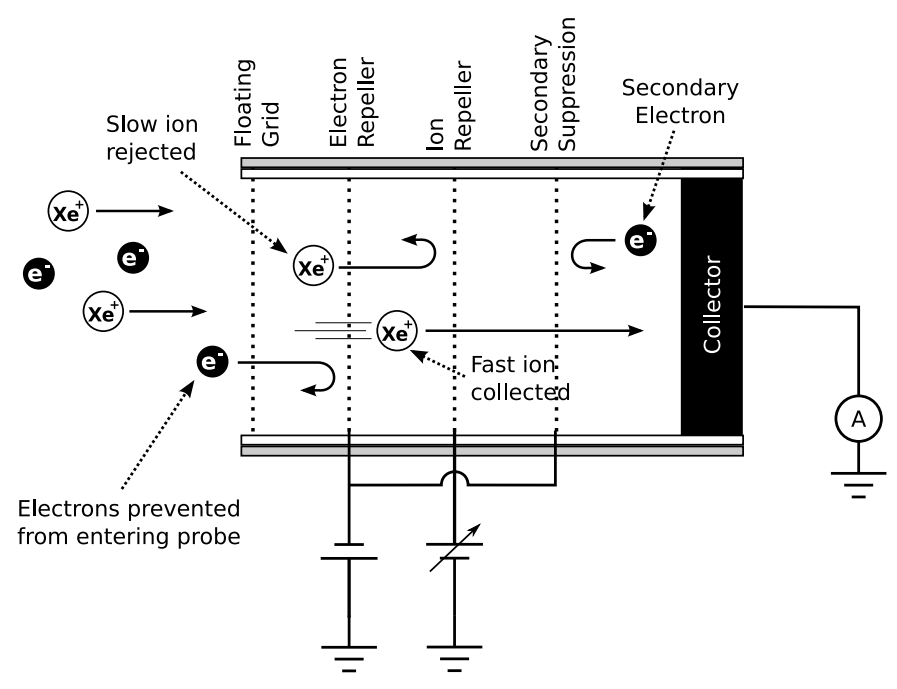

Figure 5.6: Schematic representation of an RPA

\subsubsection{Design and operation}

The RPA used in these experiments consists of a planar tungsten collector preceded by four grids. The entry grid (first on the left in Figure 5.6) is left floating and serves to shield the plasma from the potentials on the remaining grids. The next grid is an electron repelling grid, which is biased to $-15 \mathrm{~V}$ relative to ground in order to keep out plasma electrons. Technically, this grid should be biased relative to the ambient plasma potential, but the plasma potentials in the regions in which the RPA operates were small compared to $-15 \mathrm{~V}$. The next grid is the ion repeller, which is swept through positive voltages. The final grid, closest to the collector, is a second electron repeller, biased to the same potential as the first. Its function is to repel secondary electrons emitted from the collector back to the collector in order to minimize the error due to secondary electron emission.

The grids in the RPA are spaced $2.54 \mathrm{~mm}(0.1 \mathrm{in})$ from each other and are made of stainless-steel mesh. The wires in the mesh are $114 \mu \mathrm{m}(0.0045 \mathrm{in})$ in diameter and have a spacing of approximately 3.9 lines-per-mm (100 lines-per-inch). Both the orifice and the collector are circular with a diameter of $12.7 \mathrm{~mm}(0.5 \mathrm{in})$.

The RPA was powered by two supplies. The first supply was a common, DC power supply which provided the $-15 \mathrm{~V}$ repelling voltage for both electron suppression grids. A Keithley 2410 SourceMeter provided voltage to the ion repelling grid. The sourcemeter was controlled via a GPIB interface and associated software written in National Instruments LabVIEW. The current at the collector was amplified by a Femto DLPCA- 
200 current amplifier and its voltage output measured by a computerized DAQ system. Timing signals from the sourcemeter triggered the data acquisition so that the voltage sweep and current collection were synchronized. The gain on the current amplifier was initially set to $10^{5} \mathrm{~V} / \mathrm{A}$. Later experiments utilized a computer-controlled automatic gain, which found the maximum gain at which the amplifier could be operated without causing it to saturate.

The RPA was mounted on the same boom as the Faraday probe and swept from $-90^{\circ}$ to $60^{\circ}$ in $10^{\circ}$ increments. At each angle five individual traces were collected, and averaged. The resulting average trace was then further processed as described below.

\subsubsection{RPA trace smoothing}

Unfortunately, at operating conditions where the thruster was highly oscillatory, the RPA data acquired exhibited poor signal-to-noise ratios. I attempted several techniques to mitigate this problem, including varying the sweep rate, averaging multiple RPA traces, inserting a low-pass filter into the current collection path, and operating the gain at the maximum allowable value for each sweep. However, none of the techniques made a significant difference. To draw reasonable conclusions from the data, it was therefore necessary to apply a smoothing spline to the data. The data were first smoothed with the Savitzky-Golay algorithm. ${ }^{10,11}$ The implementation of this algorithm is available in Appendix A.2. A cubic spline was then applied to the smoothed data. The splines had between 10 and 40 knots, depending on the quality of the data. Each spline was manually inspected and potentially adjusted to achieve the best visual fit to the data. The smoothing algorithm served to improve the initial spline fitting, but is not directly used in the result. Figure 5.7 shows a sample I-V curve from RPA data for this work. Additionally, it shows the spline, the knots of the spline, and the resulting IEDF. The IEDF is plotted on the right-hand y-axis and, additionally, on the color bar.

To approximate the uncertainties on quantities derived from the IEDF, four additional splines are created, as shown in Figure 5.8. They are labelled "High Energy Estimator", "Low Energy Estimator", "High Spread Estimator", and "Low Spread Estimator". The High Energy Estimator spline places an upper bound on the energies in the IEDF recorded. It is drawn such that the optimal spline is offset to the right until the spline falls above all of the data in the steepest portion of the I-V curve. Likewise, the Low Energy Estimator spline is for estimating the lower bound on the IEDF and is drawn by offsetting the optimal curve to the left until it is below all of the data in the I-V curve. High Spread Estimator and Low Spread Estimator are used to estimate the narrowest and widest peaks in the IEDF that could be represented by the I-V curve recorded, respectively. The High Spread Estimator is the shallowest spline that can be drawn through the data, while the Low Spread Estimator is the steepest spline that can be drawn. 


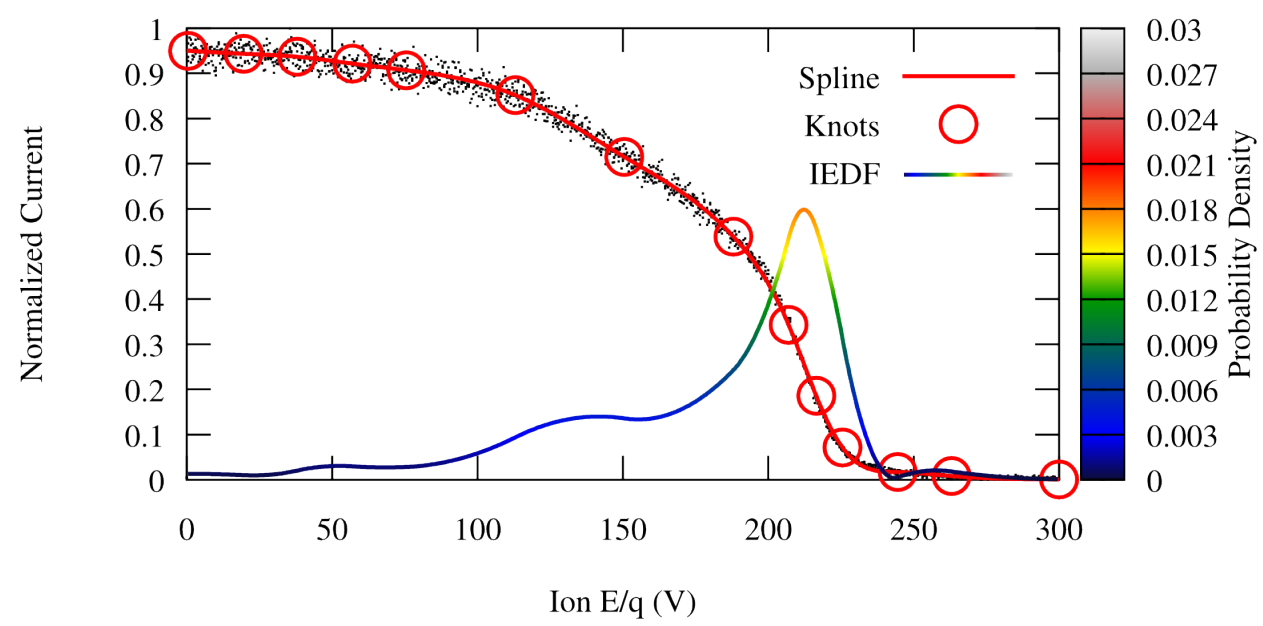

Figure 5.7: Sample RPA data, spline, and resulting IEDF

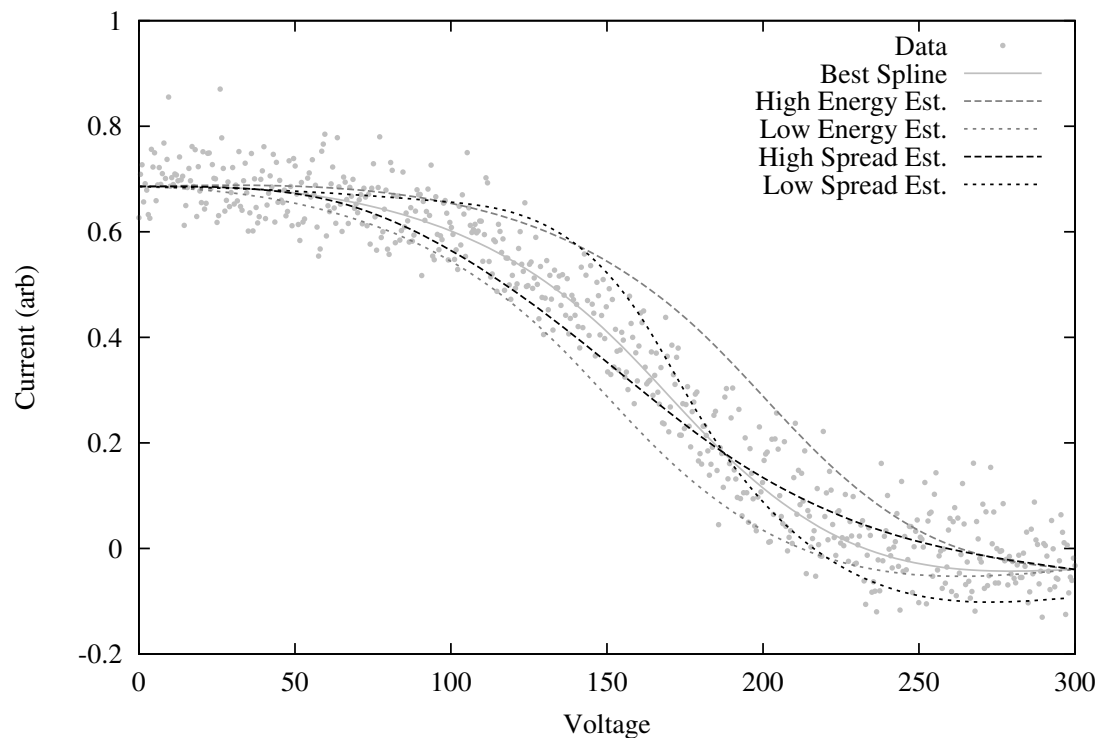

Figure 5.8: A particularly noisy RPA trace showing the splines used to estimate the uncertainty.

\subsection{Double Probe}

\subsubsection{Description and simple analysis}

Single Langmuir probes suffer from a variety of errors. First, when the probe is biased positive with respect to the plasma potential, large electron currents may be 


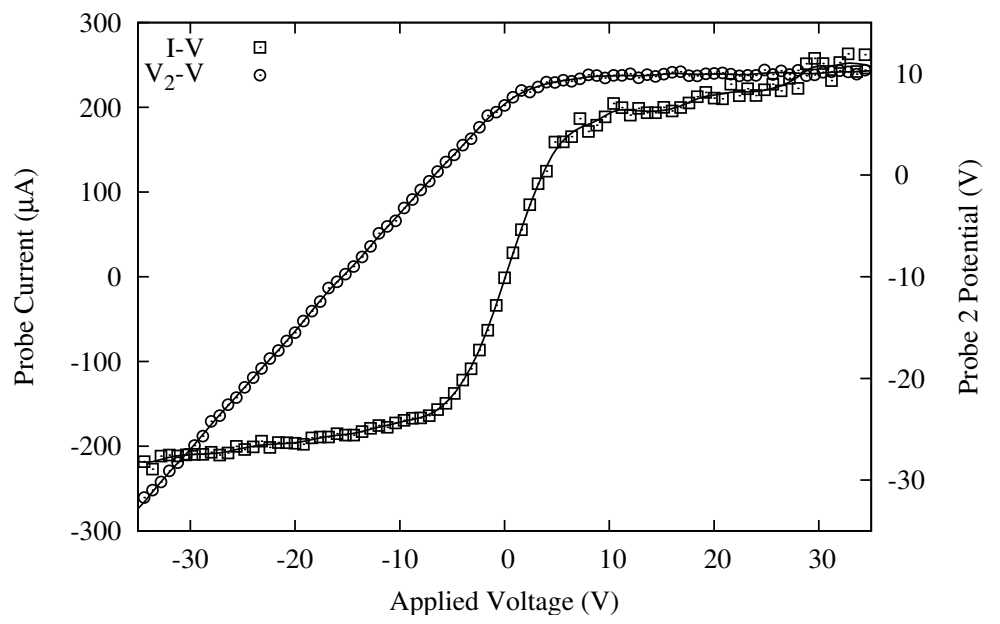

Figure 5.9: Typical double-Langmuir probe trace

drawn which disturb the plasma. Second, strong magnetic fields disturb the collection of electrons, causing errors, particularly in density measurements. Third, the saturation knees are not observed in all cases. If no knee is found in the trace, it is difficult to quantify any plasma parameters.

The double probe configuration ${ }^{12}$ completely eliminates the first and second problems, and at least partially alleviates the third. A double probe is two Langmuir probes, typically of equal size, mounted close to each other. A bias is applied between the two probes. The plasma between the two probes completes the circuit, and the current is measured for the applied voltage. A typical double probe trace is shown in the $I-V$ curve of Figure 5.9. Because both probes are of finite size, and one of the two is always biased to attract ions, the total current through the circuit is limited to the ion saturation current,

$$
I_{\text {sat }}=e n_{e} A \sqrt{\frac{k T_{e}}{m_{i}}} .
$$

To be more explicit, the two probes always bracket the floating potential-one is positive of it, and one negative. Therefore, one of the two probes must be attracting ion current. The ion current is typically several orders of magnitude smaller than the electron saturation current. Since the currents to each probe must be equal, the fact that one is limited to $I_{\text {sat }}$ limits the total current drawn by the probe.

As a result, the probe is less likely to disturb the plasma as compared to a single Langmuir probe. The expanding sheath of the probe has a smaller effect on ion trajectories (owing to their larger masses) and therefore, interferes less with the achievement of saturation. Thus, the knees of the double probe trace are more likely to be found, 
enabling robust analysis. Furthermore, because the trace of the probe is governed by the ion current, and the motion of the ions is significantly less affected by magnetic fields, the double-probe is immune to magnetic field effects while the Larmor radius for the ions remains large compared to the probe. ${ }^{13}$

Despite these advantages, the double probe has two limitations. Unlike the single probe, the double probe cannot directly measure plasma potential. In fact, the entire configuration is designed to prevent either electrode of the probe from reaching plasma potential. However, if the floating potential is measured, the plasma potential may be calculated from the electron temperature and density determined by the probe. The second limitation is that the double probe is susceptible to errors caused by gradients of the plasma. If the two electrodes are not in identical plasma environments, the resulting analysis will, at best, represent an average of the environment at each electrode. Steeper gradients may render the analysis meaningless.

If $V$ is the voltage applied between the two probes, an ideal double-probe trace takes the shape of a hyperbolic tangent given by: ${ }^{12}$

$$
I(V)=I_{i, \operatorname{sat}} \tanh \left(\frac{1}{2} \frac{e V}{k T_{e}}\right) .
$$

Often, real double probe traces do not saturate due to the expanding sheath of the probes as a voltage is applied. It is often sufficient to account for this expansion with a simple linear function. A simple analysis of the double probe current characteristic is performed by fitting the traces to the function:

$$
I(V)=a_{0} \tanh \left(a_{1} V\right)+a_{2} V+a_{3} .
$$

The parameter $a_{0}$ is simply the ion saturation current, Equation 5.7. The parameter $a_{1}$ is the multiplier in Equation 5.8, that is, $e / 2 k T_{e}$. The parameters $a_{2}$ and $a_{3}$ account for the expansion of the sheath and any small offset due to gradients in the plasma parameters, respectively. $T_{e}$ and $n_{e}$ are extracted from $a_{0}$ and $a_{1}$. The parameters $a_{2}$ and $a_{3}$ are necessary to perform the fit, but contain no information of particular interest to the final result. In addition to the I-V curve, the potential of one of the probes with respect to ground, $V_{2}$, may also be taken. When the applied voltage is zero, $V_{2}$ is at floating potential, that is, $V_{2}=V_{f}$. Using the empirical value

$$
a=0.607+\frac{2432}{\exp \left(7.01 \xi^{0.096}\right)},
$$

and given the measurements of $T_{e}, n_{e}$, and $V_{f}$ above, the plasma potential may be calculated according to ${ }^{14}$ 


$$
V p=V f+\frac{k T_{e}}{2} \log \left(\frac{m_{i}}{2 a^{2} \pi m_{e}}\right) .
$$

\subsubsection{Peterson and Talbot analysis}

The simple approach presented above is subject to errors that may arise due to sheath expansion, as discussed in Section 5.1. To overcome these problem, Peterson and Talbot derived a theoretical approach to single and double Langmuir probe traces which accounts for sheath expansion ${ }^{2}$ and has been shown to be robust. ${ }^{15}$ The method involves adjusting the plasma parameters to minimize the difference between the acquired data and the theoretical curve.

Defining the non-dimensional potential as

$$
\chi=\frac{e V}{k T_{e}},
$$

we can then define the non-dimensional potentials of each probe relative to the plasma potential, $\chi_{1}$ and $\chi_{2}$, as well as the non-dimensional floating potential, $\chi_{f}$. Note that these values will always be negative as the double probe electrodes never reach plasma potential. The non-dimensional potential difference between the probes and the floating potential are defined as

$$
\begin{aligned}
& \psi_{1}=\chi_{f}-\chi_{1} \\
& \psi_{2}=\chi_{2}-\chi_{f} .
\end{aligned}
$$

Adding Equation 5.14 to Equation 5.13 yields the non-dimensional voltage applied between the probes

$$
\psi=\psi_{2}+\psi_{1}=\chi_{2}-\chi_{1} .
$$

The current collected at each probe is given by an empirical fit to Laframboise's ${ }^{16}$ theoretical treatment of Langmuir probe dynamics:*

$$
\begin{aligned}
& I_{1}=I_{\text {sat }, 1}\left(\beta-\chi_{f}+\psi_{1}\right)^{\alpha} \\
& I_{2}=I_{\text {sat }, 2}\left(\beta-\chi_{f}-\psi_{2}\right)^{\alpha}
\end{aligned}
$$

where $\alpha$ and $\beta$ are given by

*Laframboise's theoretical treatment utilizes intensive numerical calculations to arrive at solutions. It was impractical in the 1970s when Peterson utilized them to attempt to use them directly. Today, it may be practical in some situations to use direct calculations with Laframboise theory rather than using the empirical fit. However, when processing large collections of data, as is done in this work, the empirical fit remains the most efficient option. 


$$
\begin{aligned}
& \alpha=2.900 /[\ln (\xi)+2.300]+0.070\left(T_{i} / T_{e}\right)^{0.750}-0.340 \\
& \beta=0.070+\left\{5.100+0.135[\ln (\xi)]^{3}\right\}
\end{aligned}
$$

for probes attracting ions. Recall that neither probe reaches plasma potential, so neither probe attracts electrons. With the ion currents to each probe, the total current through the circuit is given by

$$
I(\psi)=\frac{I_{1}\left(A_{2} / A_{1}\right) \exp (\psi / 2)-I_{2} \exp (-\psi / 2)}{\exp (\psi / 2)+\left(A_{2} / A_{1}\right) \exp (-\psi / 2)} .
$$

Note that this is mathematically similar to the hyperbolic tangent,

$$
\tanh (x)=\frac{\exp (x)-\exp (-x)}{\exp (x)+\exp (-x)}
$$

Equation 5.20 represents a theoretical expression for the I-V trace of a double probe given the following parameters: $T_{e}, T_{i}, n_{e}, r_{p}$, and $x_{f}$. Of these, $r_{p}$ is known, and $T_{e}$ and $n_{e}$ will be adjustable fit parameters. The non-dimensional floating potential can be determined theoretically given $T_{e}, T_{i}$ and $n_{e}$ from the implicit equation

$$
\chi_{f}=\frac{1}{2} \ln \left(\frac{m_{e}}{m_{i}}\right)+\alpha \ln \left(\beta-\chi_{f}\right) .
$$

That leaves $T_{i}$. Fortunately, in HET plasmas, as in many other plasmas, the ion temperature is much lower than the electron temperature, typically near $800 \mathrm{~K} .{ }^{17}$ Furthermore, $T_{i}$ shows up only in $\alpha$ (Equation 5.18), where it is a weak function. Therefore, an estimate is sufficient. In this work, a value of $773 \mathrm{~K}\left(500^{\circ} \mathrm{C}\right)$ is used for all analyses.

As was noted above, the floating potential with respect to ground can be easily determined from the plot of the potential of one of the probes with respect to ground versus the applied voltage. Using the knowledge of the floating potential and Equation 5.22 the plasma potential with respect to ground can be determined from the Peterson and Talbot fit to the $I-V$ trace of a double probe.

However, if the entire trace of probe potential versus applied voltage is available, as in the $V_{2}-V$ curve of Figure 5.9, this can also be used to refine the analysis of the double probe trace. Again, according to Peterson and Talbot, the potential of probe 1, that is, the probe which swings negative when a positive voltage is applied is given implicitly by

$\psi_{1}(\psi)=-\ln \left[\left(1+\frac{\psi_{1}}{\beta-\chi_{f}}\right)^{\alpha}+\frac{A_{2}}{A_{1}}\left(1+\frac{\psi_{1}}{\beta-\chi_{f}}-\frac{\psi}{\beta-\chi_{f}}\right)^{\alpha}\right]+\ln \left[1+\frac{A_{2}}{A_{1}} \exp (\psi)\right]$.

From Equation 5.15, the voltage of probe 2 is simply 


$$
\psi_{2}(\psi)=\psi-\psi_{1}(\psi) .
$$

With these equations in hand, it is possible to analyze not only the $I-V$ trace of the double probe, but also either $V_{\text {probe }}-V_{\text {applied }}$ trace and thereby increase confidence in the measurement.

\subsubsection{Algorithm}

The analysis of the double probe traces needed to be robust, as several thousand traces needed to be processed and manual intervention was undesirable. For each data point, the $I-V$ trace and the corresponding $V_{2}-V$ trace were available. The algorithm proceded as follows:

1. Average multiple traces, into a single $I-V$ and $V_{2}-V$ trace, if applicable.

2. Use Levenberg-Marquardt ${ }^{18,19}$ least-squares routine to fit the $I-V$ curve to Equation 5.9.

3. Substitute the parameters for the best-fit parameters from the preceding step into the expression for the derivative of Equation 5.9:

$$
\frac{d I}{d V}=a_{0} a_{1}\left[1-\tanh \left(a_{1} x\right)^{2}\right]+a_{2}
$$

4. Select all data points $j$ such that $\frac{d I\left(V_{j}\right)}{d V}<=0.8 \max \left(\frac{d I}{d V}\right)$.

5. Perform a linear regression on the selected data points. The resulting slope, $m_{d}$, approximates the slope of the measured $I-V$ curve at $V=0$.

6. Calculate $T_{e}$ and $n_{e}$ from $a_{0}$ and $a_{1}$ from step 2.

7. Use the $T_{e}$ and $n_{e}$ as estimates to a Nelder-Mead Simplex optimization ${ }^{20}$ which simultaneously fits the measured $I-V$ and $V_{2}-V$ curves to the Peterson and Talbot theoretical curves. Additionally, it attempts to match the slope of the line from step 5 to the slope of the same regression performed on the theoretical $I-V$ curve. At each optimization step it calculates the following for the current estimates of $T_{e}$ and $n_{e}$ for each of the applied voltages $V_{i}$ in the measured traces:

(a) The theoretical $I$ - $V$ curve, $I\left(V_{i}\right)$

(b) The theoretical $V_{2}-V$ curve, $V_{2}\left(V_{i}\right)$

(c) The best-fit slope, $m_{t}$, of $I\left(V_{j}\right)$ as in step 5 
With these data, the cost function, $C$, for the optimization is given by

$$
\begin{aligned}
C_{1} & =\frac{1}{\max \left(\left|I_{i}\right|\right)^{2}} \frac{1}{N} \sum_{i}\left[I_{i}-I\left(V_{i}\right)\right]^{2} \\
C_{2} & =\frac{1}{\max \left(\left|V_{2, i}\right|\right)^{2}} \frac{1}{N} \sum_{i}\left[V_{2, i}-V_{2}\left(V_{i}\right)\right]^{2} \\
C_{3} & =\left[\arctan \left(m_{d}\right)-\arctan \left(m_{t}\right)\right]^{2} \\
C & =0.5 C_{1}+0.2 C_{2}+0.3 C_{3} .
\end{aligned}
$$

The normalization factors $1 / \max (\ldots)$ in $C_{1}$ and $C_{2}$ and the arctangents in $C_{3}$ are included to keep the costs approximately in the range of 0 to 1 . This makes it easier to weight the individual cost components. It also makes for meaningful comparisons of costs from one dataset to the next. The arctangents also make reasonable comparisons between slopes possible, since the result is a comparison of angles rather than slopes. The cost weights, $0.5,0.2$, and 0.3 , were found through trial and error to best enable the Nelder-Mead algorithm to find the optimal values for $T_{e}$ and $n_{e}$.

8. Find $V_{f}$ relative to ground by extracting it from the $V=0$ crossing of the measured $V_{2}-V$ curve.

9. Calculate $V_{p}$ from the $T_{e}$ and $n_{e}$ determined in step 7 and Equation 5.22.

The implementation of this algorithm is available in Appendix A.1.

\subsubsection{Design and operation}

The double probes used in these experiments consisted of a double bore alumina tube approximately $300 \mathrm{~mm}$ in length with an outside diameter of $4.76 \mathrm{~mm}(3 / 16 \mathrm{in}$.) and a bore diameter of $1.6 \mathrm{~mm}$ (0.063 in.). Tungsten wire of $0.5 \mathrm{~mm}(0.02 \mathrm{in})$ diameter was fed through each bore. The end of the tube was sealed with a ceramic adhesive leaving $4 \mathrm{~mm}$ of each electrode exposed. Each electrode was approximately $4 \mathrm{~mm}$ from the other, ensuring that the probes were several Debye lengths apart. The diameter of the wire was chosen as a good compromise between maximizing $r_{p} / \lambda_{d}$ in all regions of the plume and minimizing probe size.

The probes were mounted on a 2-axis motion table capable of moving the probe axially and radially. The probe shaft was parallel to the thrust axis and at a height such that the probe crossed the center of the thruster. Using the motion table, the probes were moved in arbitrarily defined sweep patterns. The patterns used attempted to minimize the time spent in continuous exposure to dense, hot plasma as probe heating deleteriously affected the probe performance. This is further discussed in Appendix B. 


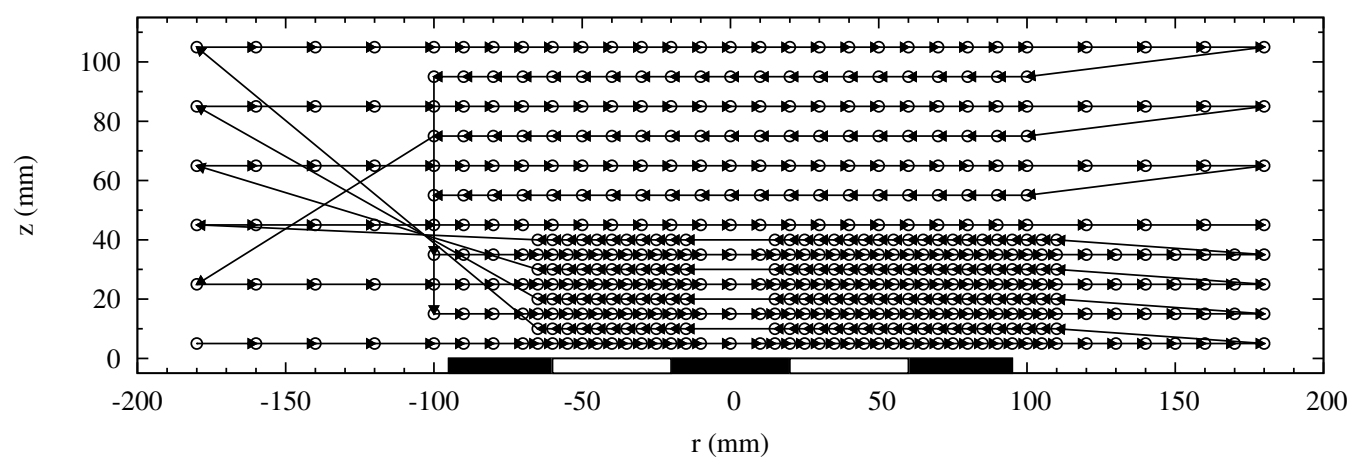

Figure 5.10: Double probe sampling pattern

The final probe pattern used is shown in Figure 5.10. This is the pattern used at cathode position $r=200 \mathrm{~mm}$. Points were removed from this pattern for cathode locations closer to the thrust axis $(r=0 \mathrm{~mm})$. At each point, the applied voltage was swept from $-40 \mathrm{~V}$ to $40 \mathrm{~V}$ in $1 \mathrm{~V}$ increments at a rate of approximately $100 \mathrm{~Hz}$. Correspondingly, each point took approximately $1.5 \mathrm{~s}$, including time to position the probe.

\section{References for Chapter 5}

[1] Langmuir, I. and Mott-Smith, H. M., "Studies of Electric Discharges in Gases at Low Pressures," General Electric Review, Vol. 27, 1924.

[2] Peterson, E. W. and Talbot, L., "Collisionless Electrostatic Single-Probe and Double-Probe Measurements," AIAA Journal, Vol. 8, No. 12, December 1970, pp. 2215-2219.

[3] Hagstrum, H. D., "Auger Ejection of Electrons from Tungsten by Noble Gas Ions," Physical Review, Vol. 96, No. 2, October 1954, pp. 325-335.

[4] Rovey, J. L., Walker, M. L. R., Gallimore, A. D., and Peterson, P. Y., "Magnetically filtered Faraday probe for measuring the ion current density profile of a Hall thruster," Review of Scientific Instruments, Vol. 77, No. 1, 2006, Paper No. 013503.

[5] Walker, M. L. R., Hofer, R. R., and Gallimore, A. D., "Ion Collection in Hall Thruster Plumes," Journal of Propulsion and Power, Vol. 22, No. 1, January 2006, pp. 205-209. 
[6] Walker, M. L. R., Victor, A. L., Hofer, R. R., and Gallimore, A. D., "Effect of Backpressure on Ion Current Density Measurements in Hall Thruster Plumes," Journal of Propulsion and Power, Vol. 21, No. 3, May 2005, pp. 408-415.

[7] Rapp, D. and Francis, W. E., "Charge Exchange between Gaseous Ions and Atoms," Journal of Chemical Physics, Vol. 37, No. 11, December 1962, pp. 26312645.

[8] Haas, J. M., Low-Perturbation Interrogation of the Internal and Near-field Plasma Structure of a Hall Thruster Using a High-speed Probe Positioning System, Ph.D. dissertation, University of Michigan, Ann Arbor, 2001.

[9] Hutchinson, I. H., "Chapter 3: Plasma particle flux," Principles of Plasma Diagnostics, Cambridge University Press, Cambridge, UK, 1987, pp. 51-86.

[10] Savitzky, A. and Golay, M. J. E., "Smoothing and Differentiation of Data by Simplified Least Squares Procedures," Analytical Chemistry, Vol. 36, No. 8, July 1964, pp. 1627-1639.

[11] Gorry, P. A., "General Least-Squares Smoothing and Differentiation by the Convolution (Savitzky-Golay) Method," Analytical Chemistry, Vol. 62, No. 3, March 1990, pp. 570-573.

[12] Johnson, E. O. and Malter, L., "A Floating Double Probe Method for Measurements in Gas Disharges," Physical Review, Vol. 80, No. 1, October 1950, pp. 5868.

[13] Brussaard, G. J. H., van der Steen, M., Carrère, M., van de Sanden, M. C. M., and Schram, D. C., "Langmuir probe measurements in an expanding magnetized plasma," Physical Review E, Vol. 54, August 1996, pp. 1906-1911.

[14] Chen, F. F., "Lecture Notes on Langmuir Probe Diagnostics," IEEE International Conference on Plasma Science, IEEE, Jeju, Korea, June 2-5, 2003.

[15] Brockhaus, A., Borchardt, C., and Engemann, J., "Langmuir Probe Measurements in Commercial Plasma Plants," Plasma Sources Science and Technology, Vol. 3, No. 4, November 1994, pp. 539-544.

[16] Laframboise, J. G., "Theory of Spherical and Cylindrical Langmuir Probes in a Collisionless, Maxwellian Plasma at Rest," Ph.D. dissertation, University of Toronto, Toronto, CA, 1966.

[17] Hargus, W. A. and Cappelli, M. A., "Laser-induced fluorescence measurements of velocity within a Hall discharge," Applied Physics B: Lasers and Optics, Vol. 72, No. 8, June 2001, pp. 961-969. 
[18] Levenberg, K., "A Method for the Solution of Certain Non-Linear Problems in Least Squares," The Quarterly of Applied Mathematics, Vol. 2, No. 2, July 1944, pp. 164-168.

[19] Marquardt, D. W., "An Algorithm for Least-Squares Estimation of Nonlinear Parameters," SIAM Journal on Applied Mathematics, Vol. 11, No. 2, 1963, pp. 431441.

[20] Nelder, J. A. and Mead, R., "A Simplex Method for Function Minimization," The Computer Journal, Vol. 7, No. 4, 1965, pp. 308-313. 


\section{Chapter 6}

\section{Magnetic Field}

\subsection{Motivation}

As discussed in Chapter 2, the magnetic field plays a critical role inside an HET. It is responsible for providing the electron impedance which creates the ion-accelerating potential. These fields are oriented primarily in the radial direction between the inner and outer walls of the discharge chamber, having their peak near the exit plane. The fields typically have a strength on the order of $50 \mathrm{mT}$ at their maximum, though several factors, including thruster scaling and overall geometry, affect the design.

It is impossible, however, to generate a field inside the thruster without also generating a field outside.* In the plume, the magnetic field will still affect the mobility of the electrons, however the behavior may be less desireable. In particular, it will affect the coupling between the cathode and the HET.

To understand how the magnetic field affects the cathode coupling, it is necessary to know the topology of the magnetic field. Because of the flexibility provided by a software model, I decided to model the field. The field data was also measured experimentally at one operating condition and compared to the model for verification.

Before moving forward, some terminology should be established. Figure 6.1 shows a cut-away view of an HET similar to the BPT-2000 with the important parts of the magnetic circuit labelled. The magnetic field is generated by the outer and possibly inner windings. (The BPT-2000 used in these experiments has no inner winding, but it is shown for generality.) The windings encircle the outer and inner cores, respectively. Sometimes the cores, particularly the outer core, is called a flux return or flux guide. The outer cores connect the back plate to the outer front pole, or outer pole, for short. The inner core connects the back plate to the inner front pole.

${ }^{*}$ One could, in theory, shield the thruster to prevent the external field from significantly expanding into the surrounding area. However, the mass required to do this makes it impractical for spaceflight. 


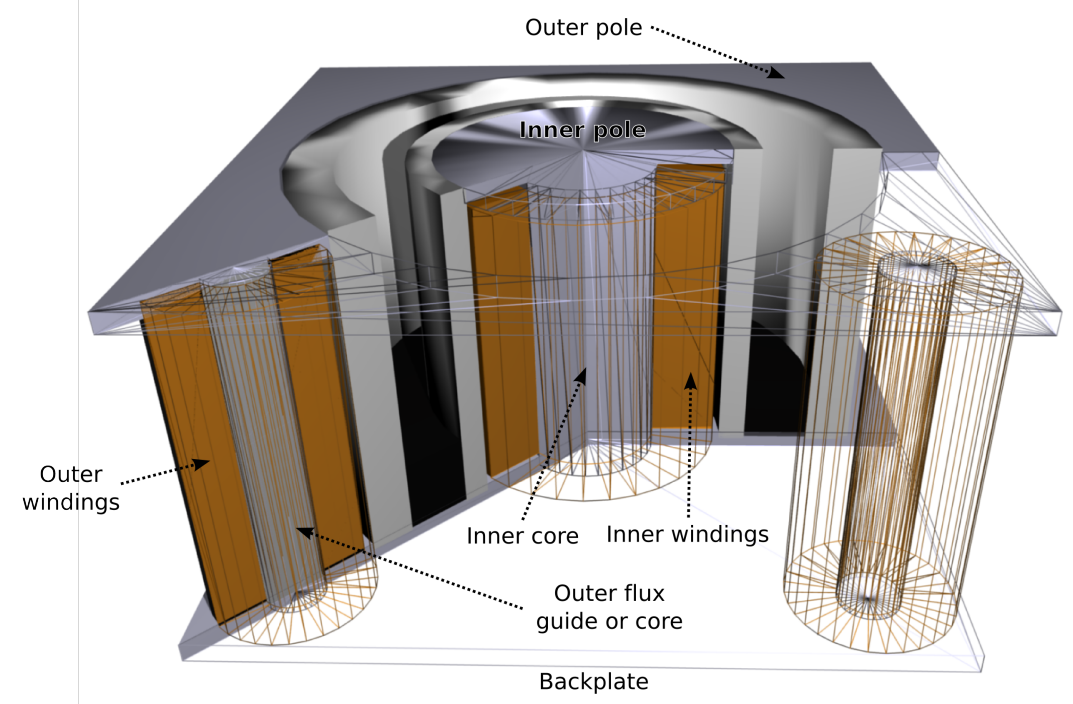

Figure 6.1: Cross section of an HET showing the parts of the magnetic circuit

\subsection{Magnetic Field Model}

\subsubsection{Model}

The thruster is not radially symmetric, and thus, a 3-D model was required. However, the thruster does exhibit eight-fold symmetry. Therefore, only one eighth of the thruster was modeled. Appropriate symmetry conditions are enforced at the volume boundaries. The model is shown in Figure 6.2. Only the magnetic circuit of the HET is included in the model. It is comprised of the outer core and windings, the inner core, the inner pole, the

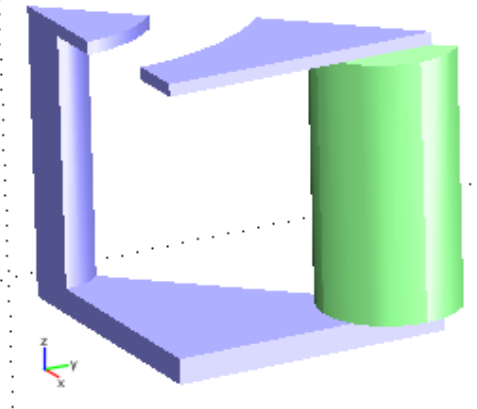

Figure 6.2: Model of the simplified HET magnetic circuit. outer pole and the back plate. The remainder of the components are non-magnetic and do not affect the field. All of the magnet components are modeled as ASTM A848 magnet iron, which is essentially pure iron. ${ }^{1}$ The magnetization curve for iron is shown in Figure 6.3. ${ }^{2}$ Similar values may be found in Chapter 3 of Bozorth's text. ${ }^{3}$ These data are incorporated into the model. The wires, which are made of copper, are modeled as free space carrying a current density determined from the number of turns in the real coils, the current applied to the coils, and the cross-sectional area of the coils according to: 


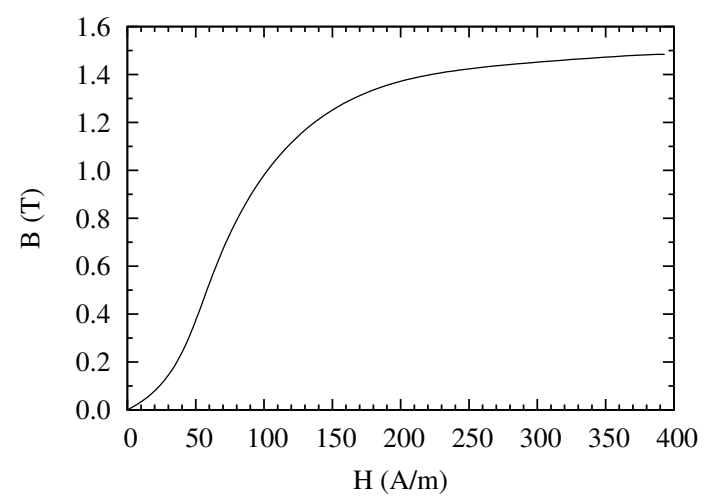

Figure 6.3: Magnetization of iron

$$
J_{\text {mag }}=\frac{I_{\text {mag }} N_{\text {turns }}}{w_{\text {coil }} h_{\text {coil }}} .
$$

The current is always circular around the center of the outer core, which is located at $r_{\text {offset }}$ in both the $x$ and $y$ directions from the center of the thruster, so the vector current density at any location in the windings is given by:

$$
\mathbf{J}=\left[\begin{array}{c}
-J_{\operatorname{mag}} \frac{y-r_{\text {offset }}}{\sqrt{\left(x-r_{\text {offset }}\right)^{2}+\left(y-r_{\text {offset }}\right)^{2}}} \\
J_{\operatorname{mag}} \frac{x-r_{\text {offset }}}{\sqrt{\left(x-r_{\text {offset }}\right)^{2}+\left(y-r_{\text {offset }}\right)^{2}}} \\
0
\end{array}\right] .
$$

The model and the surrounding vacuum was meshed with approximately 50,000 tetrahedra and the field solved. A cross section of the field along the x-axis, the plane in which the cathode sits, was extracted.

\subsection{Model Results}

The magnetic field model results are shown in Figures 6.4 and 6.5. Saturation of the iron was not observed up to $4.2 \mathrm{~A}$ of magnet current, the highest current used in any of the experiments. Without saturation, the magnetic field is linear with coil current. Therefore, the plot shows magnet field flux per ampere of magnet current (mT/A). The first significant observation is that no field line crossing likely cathode positions outside the thruster will carry an electron into, or even near, the discharge channel. All of the field lines terminate at the thruster face, or the side of the face. Furthermore, the field lines clearly show that the electrons emitted from a cathode near the thruster, for instance at $(r=100 \mathrm{~mm}, z=0 \mathrm{~mm})$ will be guided by the field lines (if, indeed, they are 
bound to these lines) either toward the outside edge of the outer pole, or around to the back plate. Finally, note that as the cathode is moved from this position in increasing $r$ or $z$, the closest approach to the thrust axis $(r=0)$ of the field line intersecting the cathode decreases.

None of the field lines crossing external locations reach the discharge chamber because of the field separatrix. A separatrix is an asymptote that the field lines may approach, but not cross. The geometry of this Hall thruster is such that the device has one separatrix line, and one separatrix surface. The separatrix line is trivial and coincides with the axis of the thruster. The separatrix surface starts at the outer edge of the face of the thruster, and up away from the face and in toward the thruster axis. Figure 6.6 illustrates the magnetic field lines and a cross-section of the separatrices for the BPT2000. As will be shown in Chapter 8, this separatrix surface plays an important role in the cathode coupling process.

\subsection{Validation of the Model}

To test the model, I conducted a survey of the magnetic field of the actual thruster. The thruster was mounted on a platform in the vacuum chamber. A current of $4.2 \mathrm{~A}$ was applied to the magnet coils. This value was chosen as it was the highest current used in the experiments. A higher current, and therefore stronger field, was desireable to increase the signal-to-noise ratio of the measurements. A single-axis, gauss probe was mounted on the two-axis motion table and aligned such that it measured the radial component of the magnetic field. The probe was swept through a grid with points spaced $10 \mathrm{~mm}$ apart when within approximately $100 \mathrm{~mm}$ of the thruster and spaced $20 \mathrm{~mm}$ apart beyond that as shown in Figure 6.7b. At each point, 1000 data points were read at a rate of $5 \mathrm{kHz}$ from an analog output of the guassmeter. These points were averaged. The magnetic field generated by the motors of the motion table interfered with the measurements. Therefore, the power to the motors was disabled while the field data were acquired. After completing the entire grid, the probe was remounted such that it measured the axial component, and the grid repeated. The gaussmeter was accurate to $0.1 \mathrm{mT}$.

Figure 6.7 shows the modeled and measured B. The measured and the modeled show good agreement with each other at distances of approximately $150 \mathrm{~mm}$ from the edges of the thruster. Outside of this region, the experimental data begins to diverge more drastically from the modeled data. There are three likely reasons for this. First of all, at these distances, the field strength is down to $0.5 \mathrm{mT}$ or less. This nears the resolution of $0.1 \mathrm{mT}$ of the gaussmeter. Furthermore, at these distance, particularly in the radial direction, the gauss probe nears the edge of the tank and other equipment mounted in the tank. These, of course, were not modeled and may have perturbed the field in unexpected ways. Finally, the magnetic properties of the actual iron are not 
known. While the material is made of ASTM-A848, the machining and handling of the material, such as how it is rolled and otherwise processed, can have an effect on the field. Aerojet was unable to provide any details of the maching process, and thus, the B-H curve used in the models cannot be expected to be accurate. Regardless of the differences at small field strengths, the most important similarity is that of the topology in general, and particularly of the separatrix. As Figure 6.7 shows, the separatrix is nearly identical between the measured field and the modeled field, and the topology is similar.

\subsection{Larmor Radius and Hall Parameter}

From the magnetic field data and estimates of the neutral density and electron temperatures we can calculate the Hall parameter and Larmor radius in the plasma. These values show the degree to which the magnetic field affects the plasma. Figure 6.8 shows the magnetic field at $2.5 \mathrm{~A}$ of magnet current (a typical value used in these experiments) along with the associated Larmor radius, neutral density, and Hall parameter plots. Each of these will be developed in the proceding sections.

\subsubsection{Larmor radius}

The Larmor radius describes the radius of the turn which a free electron makes in a magnetized plasma. The Larmor radius determines the length scale over which magnetic effects are important. If the system under consideration is much larger than the Larmor radius, then the magnetization of the plasma will be important, assuming that the Hall parameter is also high. Likewise, if the scale of the system is much smaller than the Larmor radius, then magnetization is not important in the system, regardless of the Hall parameter.

For an electron with a velocity perpendicular to the magnetic field of $v_{\perp}$, the Larmor radius is given by:

$$
r_{L}=\frac{m_{e} v_{\perp}}{e B} .
$$

Every electron has its own $r_{L}$ based on its own energy. However, for our present purposes, an estimate of the average Larmor radius based on the electron temperature is sufficient. For this, we will substitute the average thermal velocity of electrons with temperature $T_{e}$.

$$
\begin{aligned}
v_{\perp} & \approx \sqrt{\frac{2 k T_{e}}{m_{e}}} \\
\Rightarrow \bar{r}_{L} & \approx \frac{1}{e B} \sqrt{m_{e} k T_{e}}
\end{aligned}
$$




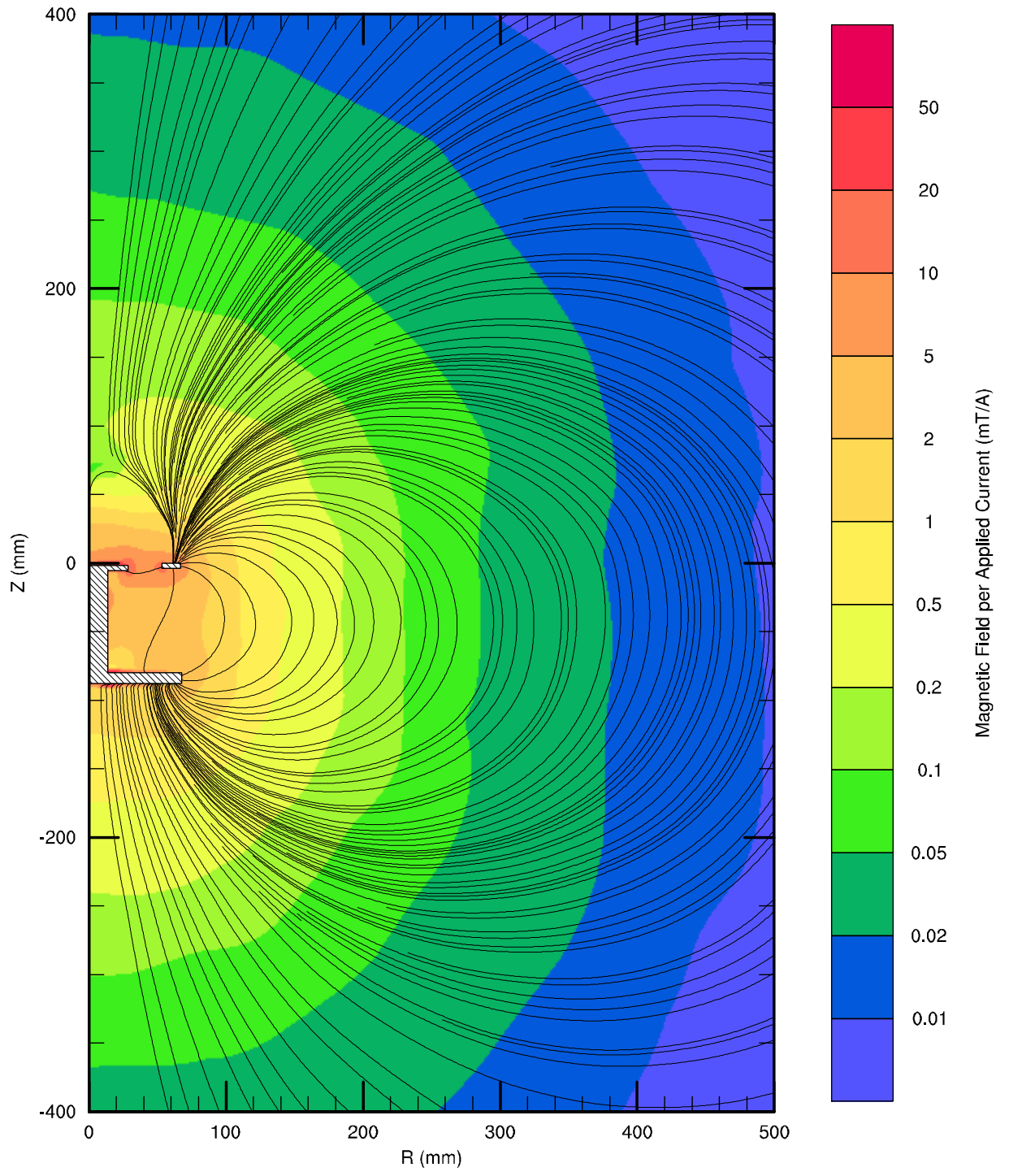

Figure 6.4: Model of the magnetic field structure of the BPT-2000 


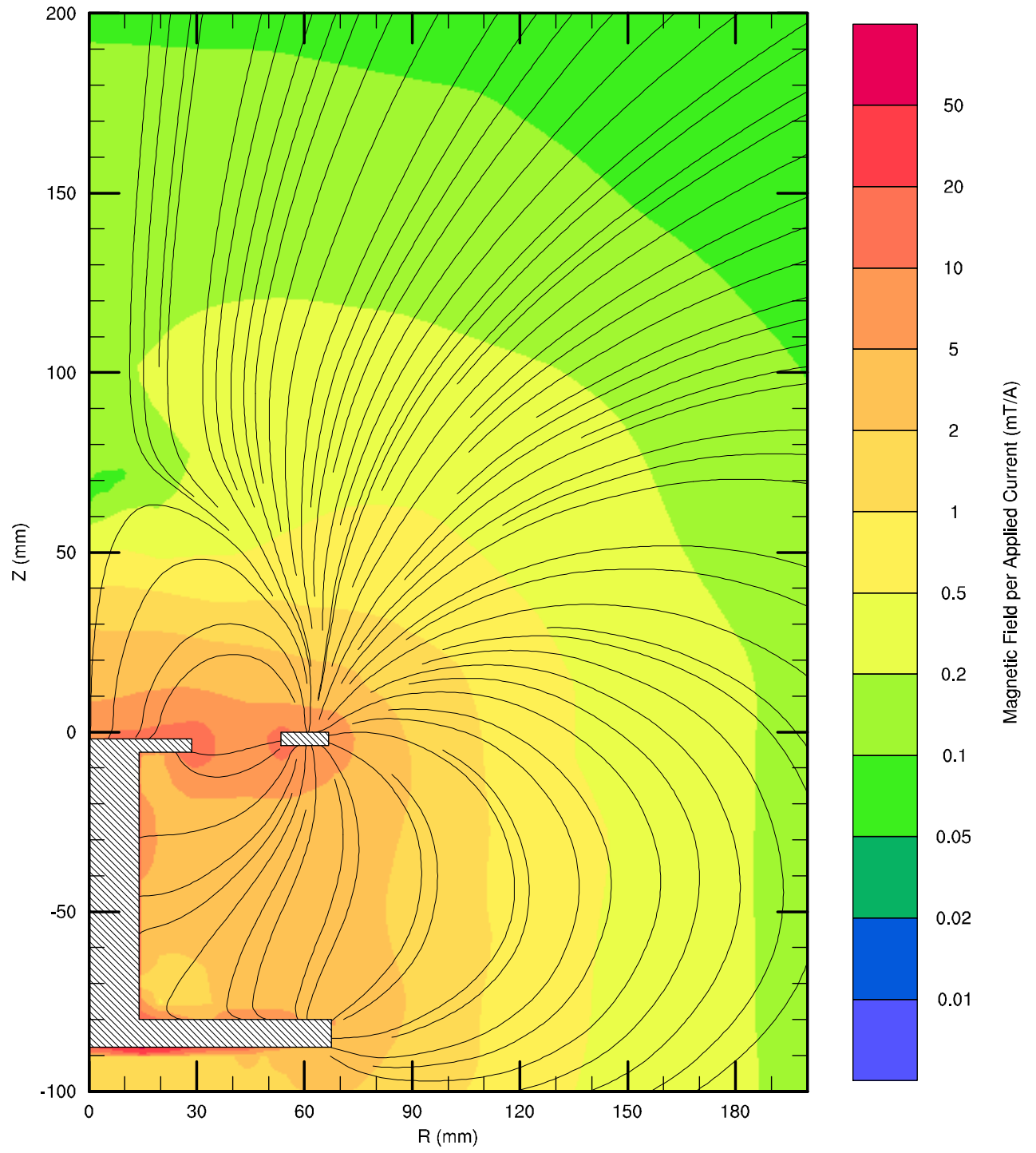

Figure 6.5: Close-up of the anode region of the magnetic model 


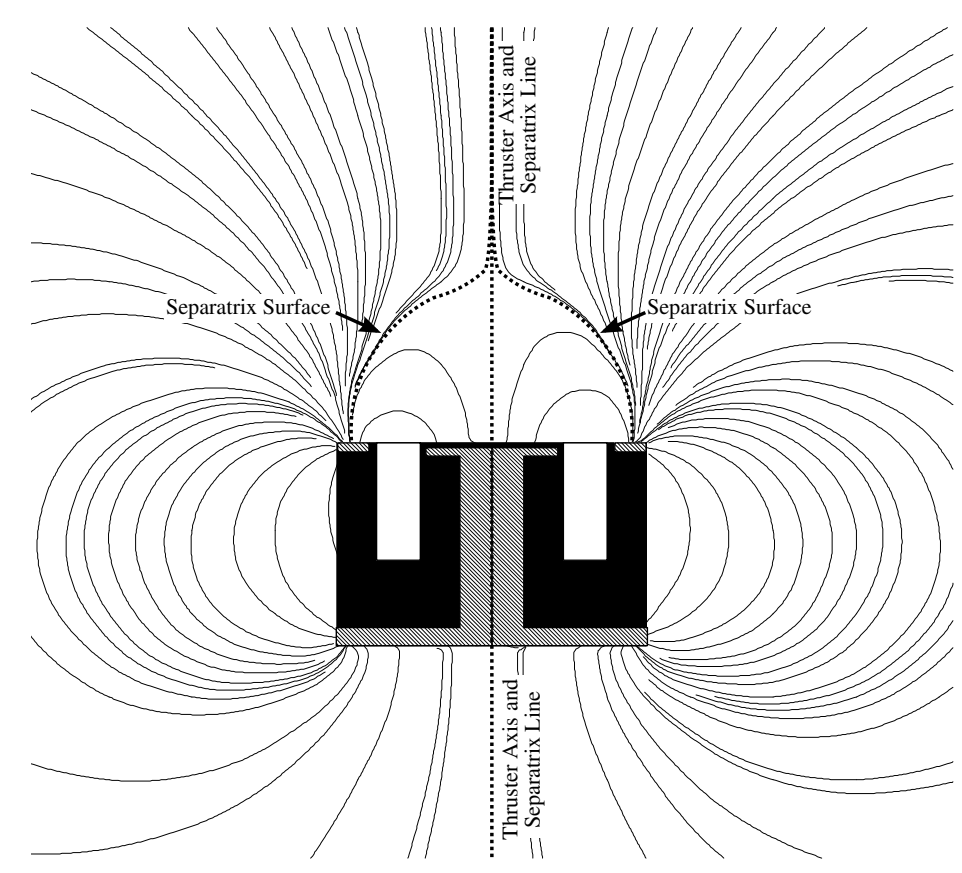

Figure 6.6: Magnetic field lines and separatrices for a typical HET.

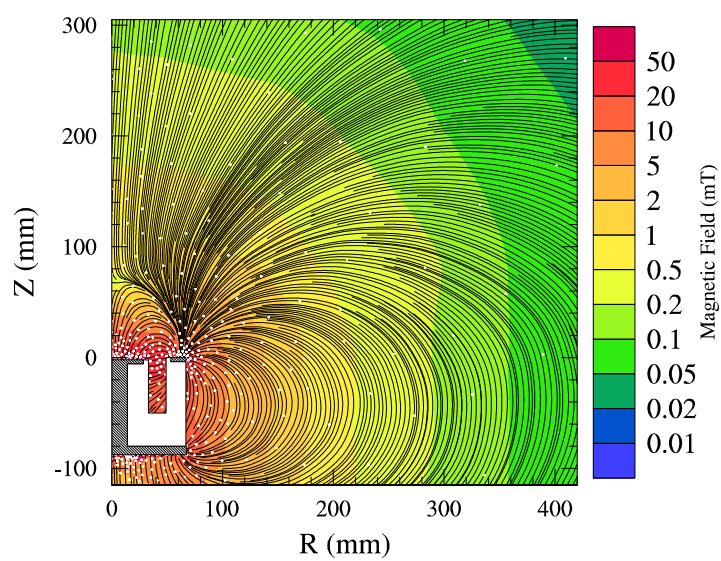

(a) Model

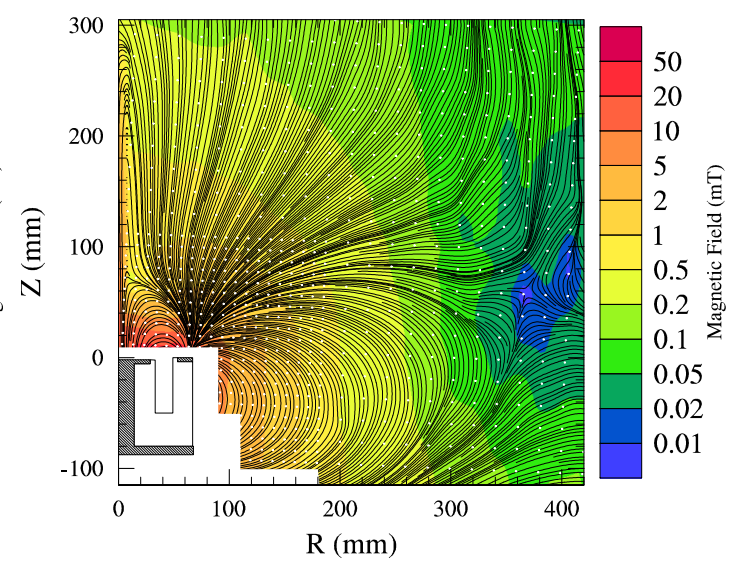

(b) Measured

Figure 6.7: Comparison of the modeled and measured magnetic fields with $4.2 \mathrm{~A}$ of magnet current

Figure $6.8 \mathrm{~b}$ shows a plot of the average Larmor radius for the field shown in Figure $6.8 \mathrm{a}$ assuming an electron temperature of $3 \mathrm{eV}$. This temperature is typical of HET plumes, as will be shown later. Naturally, a true HET plume has some variation in $T_{e}$, 


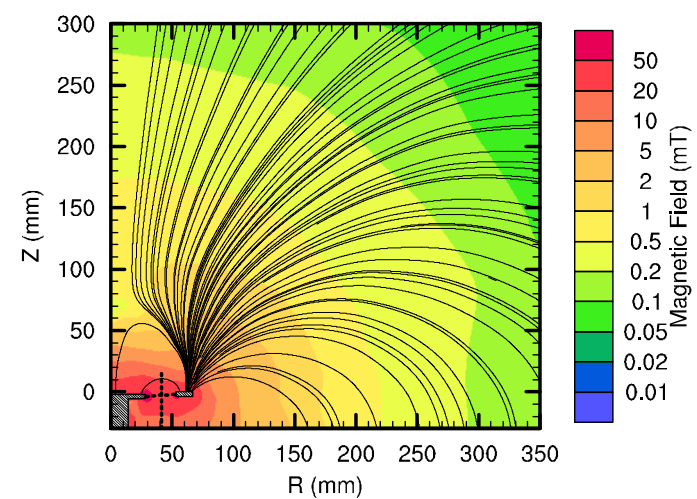

(a) Magnetic Field $\left(I_{\text {mag }}=4.2 \mathrm{~A}\right)$

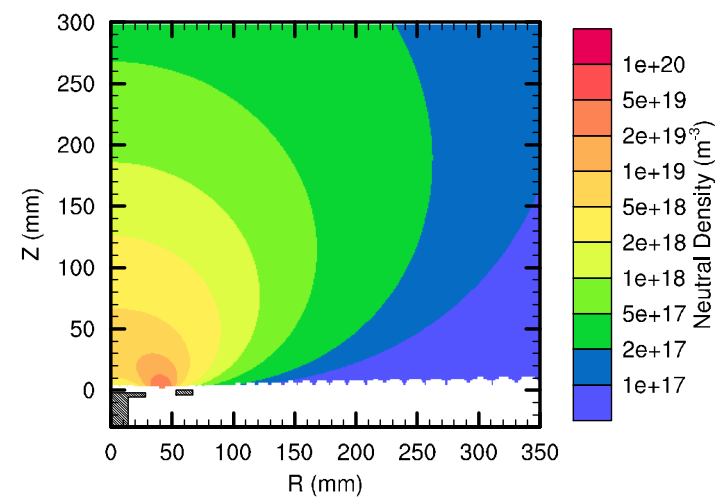

(c) Neutral Density

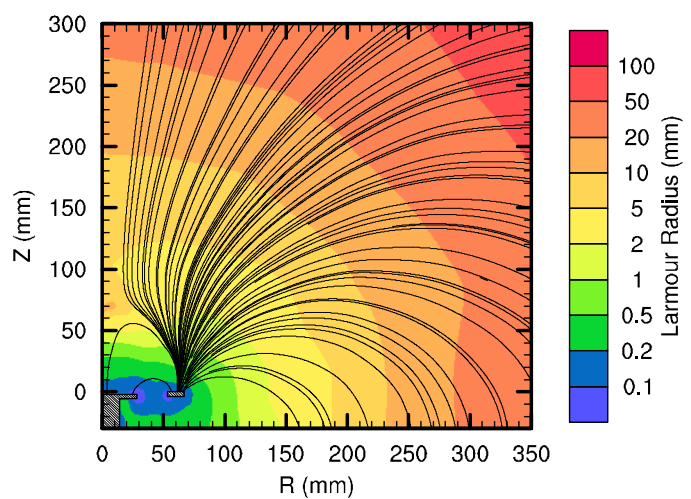

(b) Larmor Radius

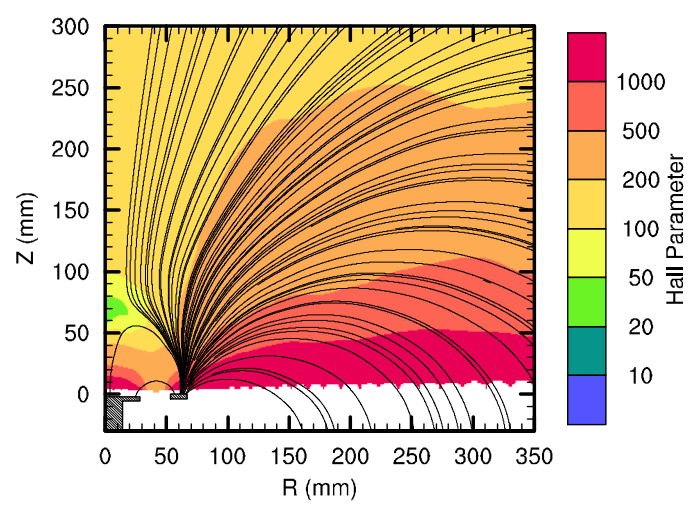

(d) Hall Parameter

Figure 6.8: Inputs and outputs used in estimating the magnetization of the plasma near the thruster

but it is typically less than an order of magnitude and not important for the present purposes.

\subsubsection{Hall parameter}

The Hall parameter, first introduced in Section 2.1.5, is a dimensionless parameter that describes the extent to which a plasma is magnetized. High values, say $>100$, correspond to plasmas for which magnetic processes dominate the electron transport behavior, whereas low values, say $<0.01$, represent plasmas where collisional processes dominate the electron transport. For magnetized, high Hall parameter plasmas, the electron current is predominately in the $\mathbf{E} \times \mathbf{B}$ direction, whereas for unmagnetized, low Hall parameter plasmas the current is largely in the direction of $\mathbf{E}$ [4, p. 82]. For Hall parameters near unity, neither one mechanism nor the other dominates. 
For the proceeding discussion, the velocity distribution is assumed to be Maxwellian. From the rate coefficient, and the neutral density, the collision rate is calculated according to

$$
v_{e}=\left\langle\sigma_{n e}\left(T_{e}\right) v_{e}\right\rangle n_{0}+n_{i}\langle<\rangle v>\sigma_{\text {Coul }}\left(T_{e}, n_{e}\right)
$$

The neutral density is difficult to measure experimentally. For large volumes, ionization gauges may be used. However, for density variations over the size of an HET this is not practical. Optical methods such as optical absorption spectroscopy and laserinduced fluorescence are capable of measurements on these smaller scales. However, they are elaborate and expensive and can only yield absolute densities with great difficulty.

Therefore, the use of analytical models is in order. To estimate the neutral density downstream of the thruster an analytic expression provided by Cai and Boyd is used. ${ }^{5}$ The expression is valid for circular and annular orifices. According to Cai and Boyd, the neutral density downstream of the orifice is given by the following expression. Given the temperature and bulk velocity of the gas at the orifice, $T_{0}$, and $U_{0}$, respectively, and

$$
\begin{gathered}
\beta=\frac{1}{2 R T_{0}} \\
Q(r, z, \rho, \theta)=\frac{z^{2}}{z^{2}+r^{2}+\rho^{2}-2 r \rho \sin \theta} \\
K(r, z, \rho, \theta)=\frac{Q^{2} U_{0}}{\beta} \exp \left(-\beta U_{0}^{2} Q\right)+\left(\frac{Q}{2 \beta}+Q^{2} U_{0}^{2}\right) \sqrt{\frac{\pi Q}{\beta}\left[1+\operatorname{erf}\left(U_{0} \sqrt{\beta Q}\right)\right]}
\end{gathered}
$$

then for an annular orifice with an inner and outer radii of $R_{1}$ and $R_{2}$, respectively, the plume density relative to the density at the orifice exit, $n_{00}$ is given by

$$
\frac{n_{0}(r, z)}{n_{00}}=\frac{1}{z^{2}}\left(\frac{\beta}{\pi}\right)^{3 / 2} \int_{-\pi / 2}^{\pi / 2} d \theta \int_{R_{1}}^{R_{2}} d \rho \exp \left(-\beta U_{0}^{2} \frac{\rho^{2}+r^{2}-2 r \rho \sin \theta}{z^{2}+\rho^{2}+r^{2}-2 r \rho \sin \theta}\right) \rho K
$$

Following Cai, ${ }^{6}$ I assumed a sonic exit velocity

$$
c_{\text {sound }}=\sqrt{\frac{\gamma k T}{m}}
$$

where $\gamma=1.66$ and $m=131.29$ amu for xenon. A temperature of $500^{\circ} \mathrm{C}$ is assumed for the neutrals at the exit. Since the mass flow and the exit plane area are fixed, we can calculate the density at the exit plane if we know the propellent utilization, $f_{p}$ : 


$$
\begin{aligned}
\dot{n} & =n_{00} A U_{0} \\
\dot{n} & =\dot{m} *\left(1-f_{p}\right) * / m \\
U_{0} & =c_{\text {sound }} \\
\Rightarrow n_{00} & =\frac{\dot{m} *\left(1-f_{p}\right)}{m A c_{\text {sound }}} .
\end{aligned}
$$

The propellet utilization is the fraction of ions introduced at the anode that are ionized. A reasonable value for HETs is $0.8 .^{7}$ Equations 6.10 and 6.15 were implemented in a Python software routine. The code is included in Appendix A. The results are shown in Figure $6.8 \mathrm{c}$ for a $4 \mathrm{mg} / \mathrm{s}$ of xenon mass flow rate assuming an ionization fraction of 0.8 .

Because of quasi-neutrality, $n_{i} \approx n_{e}$ in Equation 6.6. The electron density itself, is estimated by assuming an ionization fraction everywhere of 0.1 , that is, $n_{e} / n_{0}$. (By comparing Figure 6.8c with the electron densities presenting in Appendix $\mathrm{C}$ one can see that this is a reasonable approximation for the present order-of-magnitude analysis.) With knowledge of the magnetic field, as in Figure 6.8a, and the neutral density as in Figure 6.8c, an estimate for $n_{i}$ and again, assuming an electron temperature of $3 \mathrm{eV}$, the Hall parameter is calculated according to Equation 2.11. The results are shown in Figure 6.8d.

\subsection{Discussion}

From Figure $6.8 \mathrm{~b}$ we see that the Larmor radius at any given position is much less than the distance between that position and the thruster. Only beyond $r \approx 300 \mathrm{~mm}$ and $z \approx 250 \mathrm{~mm}$ does $r_{L}$ even reach $10 \%$ of the distance to the thruster. For $r \lesssim 250 \mathrm{~mm}$ and $z \lesssim 200 \mathrm{~mm}$ the Larmor radius is less than $10 \mathrm{~mm}$. Clearly magnetization effects, if present, will be important over the scale of our problem. From Figure 6.8d we see that the Hall parameter is everywhere greater than 20, suggesting that magnetization effects are important. However, there are some regions. Based on this analysis, we can expect from these results that magnetic effects will generally be important in the coupling processes between the cathode and the anode.

\subsection{Other HET Designs}

It should be noted that the position of the separatrix surface may be highly variable, or altogether non-existent. In particular, many HETs have an axisymmetric design. While the BPT-2000 and many other thrusters have multiple individual magnet coils connecting the outer pole to the base plate of the thruster (the BPT-2000 has four), a fully annular design has an outer flux return which is an annulus that connects the outer 
pole to the base plate. Typically these thruster have a coil wrapped around the inner core (an "inner coil"), and then a second coil either wrapped around the outside of the flux guide (an "outer coil"), or residing just inside the outer flux return (a "middle coil"). The NASA 173 HET used in much of Hofer's work has an inner and a middle coill, $^{8}$ while, for example, the T-220 has an inner and an outer coil. ${ }^{9,10}$

Figure 6.9 shows the modelled magnetic field topology resulting from a fully annular analog of the BPT-2000. Rather than having four individual magnet coils and iron cores around the outside, the thruster has an annular outer flux guide. To the outside of the flux return is an outer coil, and immediately to the inside is the middle coil. Around the inner core is the inner coil. This design has by no means been confirmed to actually function as an HET. However, it captures important topological information and allows for the exploration of magnetic field structures. Figure 6.9a is the closest fully annular analog to the actual BPT-2000. Here the outer coil contains current running out of the page, the middle coil contains current running into the page, and no current is applied to the inner coil. This is like taking an axial cross-section along the diagonal of the original BPT-2000, thus intersecting both the thruster axis and one magnet axis, and then rotating that cross-section around the thruster axis. One notes a very similar field structure as compared to Figure 6.4. If the inner current is moved from the middle coil to the inner coil, as in Figure 6.9b, very little change in the field structure is evident outside the thruster, though inside the discharge chamber there is a difference. The setup in Figure 6.9c is identical to that of Figure 6.9b, except that the current in the outer coil has been reduced. Note that this drastically affects the position of the separatrix, which now begins radially outward from the thruster off of the outer corner of the outer pole. In fact, by continuing to reduce the outer magnet current, the separatrix can be moved around, and then completely behind the thruster, until it disappears altogether. Finally, Figure $6.9 \mathrm{~d}$ shows the configuration with only the inner and the middle coils powered, both in the same direction. This is identical to the configuration of the NASA 173. In this configuration there is no separatrix surface at all. 


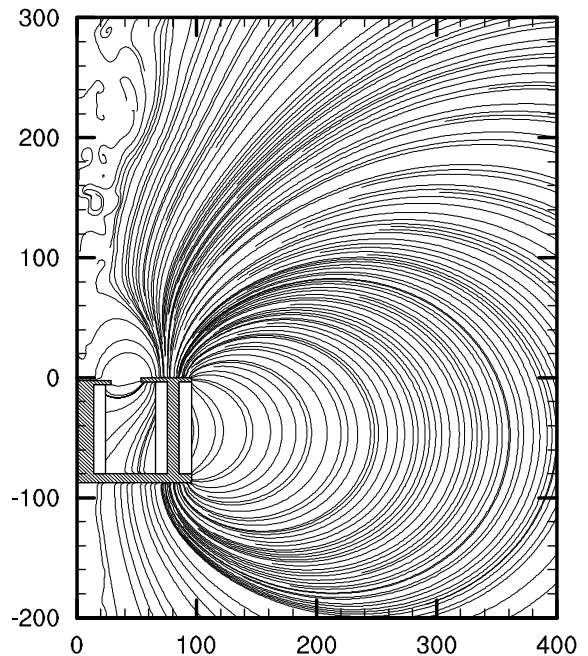

(a) $I_{\text {in }}=0 \mathrm{~A}, I_{\text {mid }}=-2.5 \mathrm{~A} I_{\text {out }}=2.5$

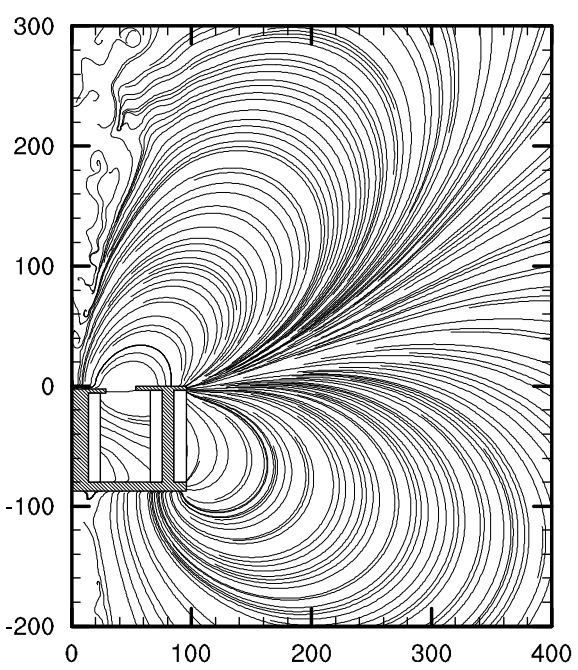

(c) $I_{\text {in }}=-2.5 \mathrm{~A}, I_{\text {mid }}=0 \mathrm{~A} I_{\text {out }}=0.3 \mathrm{~A}$

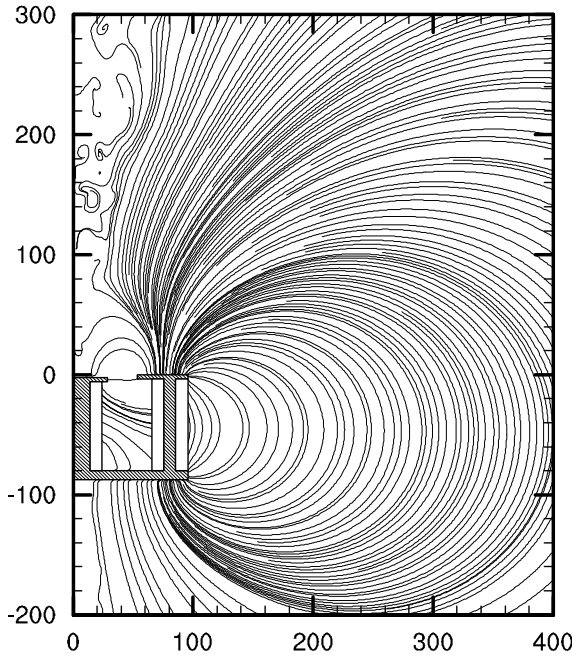

(b) $I_{\text {in }}=-2.5 \mathrm{~A}, I_{\text {mid }}=0 \mathrm{~A} I_{\text {out }}=2.5 \mathrm{~A}$

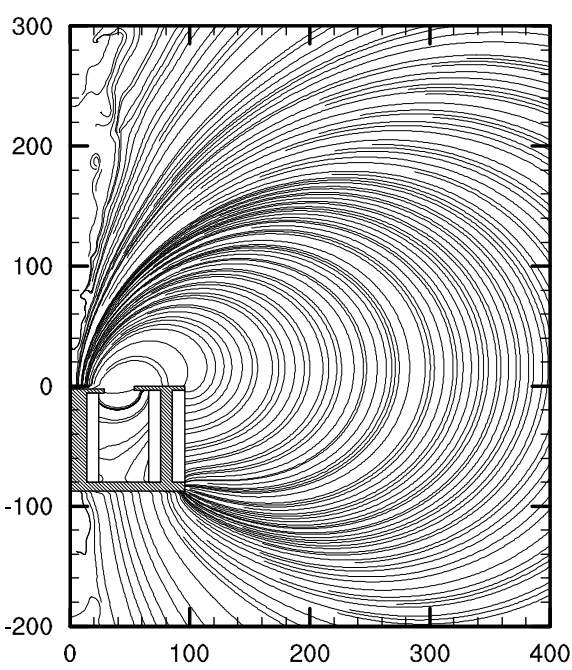

(d) $I_{\text {in }}=-2.5 \mathrm{~A}, I_{\text {mid }}=-2.5 \mathrm{~A} I_{\text {out }}=0$

Figure 6.9: Axisymmetric HET topology with different currents applied to the inner $\left(I_{\text {in }}\right)$, middle $\left(I_{\text {mid }}\right)$ and outer $\left(I_{\text {out }}\right)$ coils 


\section{References for Chapter 6}

[1] ASTM Standard A848, ASTM, First published in 1985.

[2] Clarke, R., "Magnetic properties of materials," (online) http://info.ee.surrey.ac.uk/Workshop/advice/coils/mu/. Accessed August 2008.

[3] Bozorth, R. M., Ferromagnetism, IEEE Press, Piscataway, NJ, 1978.

[4] Jahn, R. G., Physics of Electric Propulsion, McGraw-Hill Series in Missile and Space Technology, McGraw-Hill Book Company, New York, 1968.

[5] Cai, C. and Boyd, I. D., "Collisionless Gas Expanding into Vacuum," Journal of Spacecraft and Rockets, Vol. 44, No. 6, November 2007, pp. 1326-1330.

[6] Cai, C., Theoretical and Numerical Studies of Plume Flows in Vacuum Chambers, Ph.D. dissertation, University of Michigan, Ann Arbor, MI, 2005.

[7] Ashkenazy, J., Raitses, Y., and Appelbaum, G., "Parametric studies of the Hall current plasma thruster," Physics of Plasmas, Vol. 5, No. 5, 1998, pp. 2055-2063.

[8] Hofer, R. R., Development and Characterization of High-Efficiency, High-Specific Impulse Xenon Hall Thrusters, Ph.D. dissertation, University of Michigan, Ann Arbor, MI, 2003.

[9] McVey, J. B., Britt, E. J., Engleman, S. F., Gulczinski, F. S., Beiting, E. J., and Pollard, J. E., "Characteristics of the T-220HT Hall-Effect Thruster," 39th AIAA/ASME/SAE/ASEE Joint Propulsion Conference, Huntsville, AL, July 20-23 2003, Paper No. AIAA-2003-5158.

[10] Walker, M. L. R., Georgia Tech, Personal communication (electronic mail), March 16, 2009. 


\section{Chapter 7}

\section{Preliminary Experiments}

\subsection{Cathode Position Experiment}

To quantify the effect of cathode position on thruster performance, I ran the BPT2000 mounted on the thrust stand, while moving the cathode through radial and axial sweeps. Data acquisition hardware and software was used to record thrust, anode current, mass flow, and cathode coupling voltage. Thruster efficiency was calculated by the software according to Equation 2.20. For these experiments xenon was used as the propellant and cathode gas.

The cathode was mounted on a two-axis motion table as shown in Figure 7.1. This setup enabled me to place the cathode at radial displacements of $104 \leq r \leq 350 \mathrm{~mm}$ where $104 \mathrm{~mm}$ was as close to the thruster as the cathode could be positioned without contacting it (see close-up in Figure 7.1). At radial displacements greater than or equal to $154 \mathrm{~mm}$ the cathode could be positioned at axial displacements of $-295 \mathrm{~mm} \leq z \leq$ $900 \mathrm{~mm}$ where at $z=0$ the cathode face is even with the thruster face.

Several radial and axial sweeps were performed at varying thruster operating conditions. Sweeps were typically conducted from $104 \leq r \leq 304 \mathrm{~mm}$ in $10 \mathrm{~mm}$ increments. Table 7.1 shows the complete list of operating conditions. All conditions were performed with cathode mass flow rates of $\sim 1 \mathrm{mg} / \mathrm{s}(10 \mathrm{SCCM})$ and a cathode angle of zero degrees.

The thruster was run according to the standard procedure listed in Section 4.3. To acquire the thrust data, the thruster was run for two to three minutes at each of the cathode positions and the thrust data averaged over this period. The positions were selected in random order to avoid correlations between any temporal changes in thruster or thrust stand performance with changes due to cathode position. During these experiments, the auto-leveling of the thrust stand was not fully functional. Therefore, after all positions were measured, the thruster was turned off and any non-zero thrust measured 

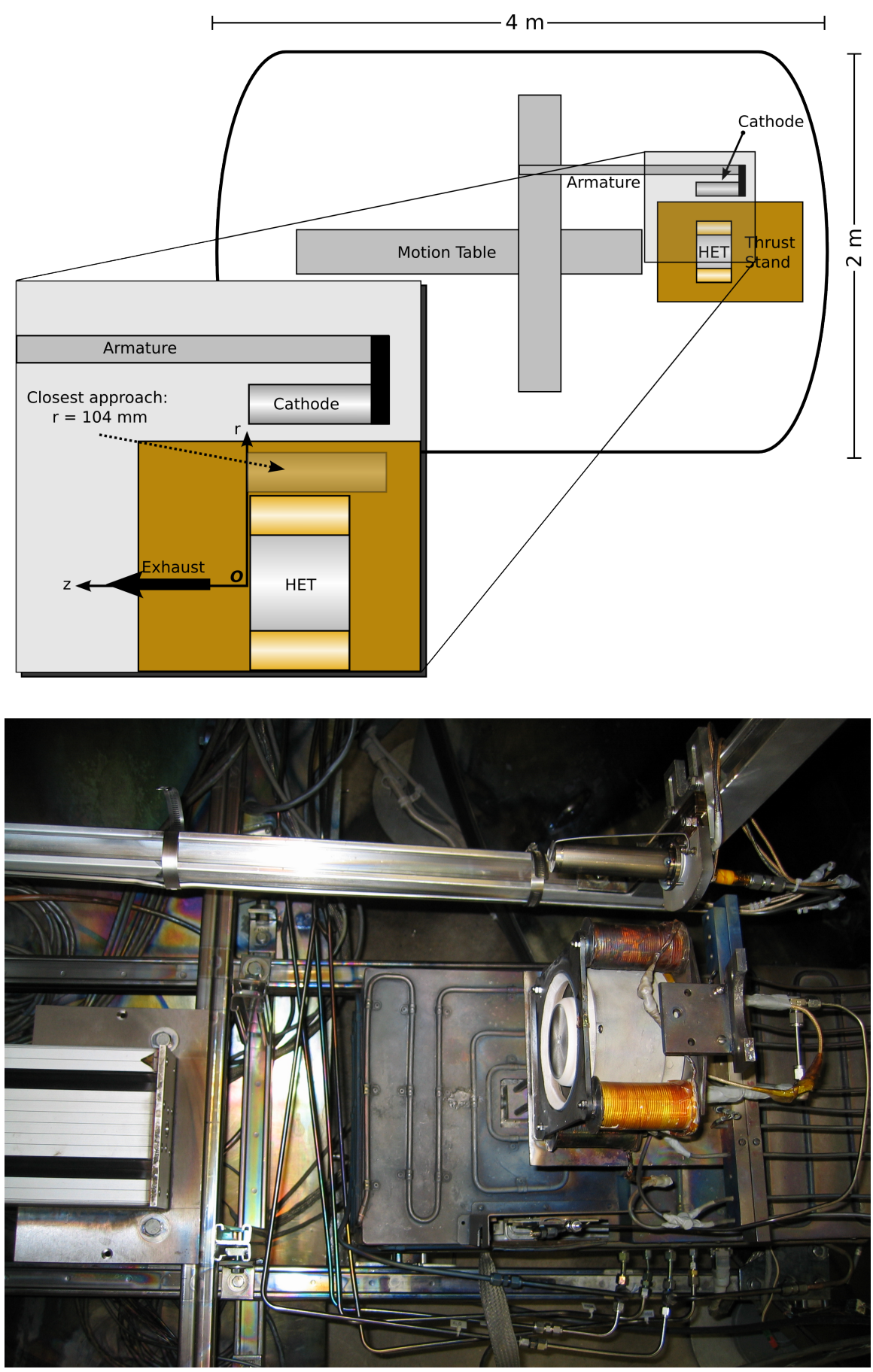

Figure 7.1: Experimental setup 
Table 7.1: Cathode Position Tests

\begin{tabular}{ccccccc}
\hline Test & $V(\mathbf{V})$ & $\dot{m}(\mathbf{m g} / \mathbf{s})$ & $I_{\text {mag }}(\mathbf{A})$ & Optimization & $\mathbf{r}(\mathbf{m m})$ & $\mathbf{z}(\mathbf{m m})$ \\
\hline Test 1a & 300 & 5.0 & 4.20 & Optimal & $104 \leq r \leq 304$ & 0 \\
Test 1b & 300 & 5.0 & 3.15 & Suboptimal & $104 \leq r \leq 304$ & 0 \\
Test 1c & 300 & 5.0 & 5.25 & Superoptimal & $104 \leq r \leq 304$ & 0 \\
Test 2a & 250 & 4.0 & 2.5 & Optimal & $104 \leq r \leq 304$ & 0 \\
Test 2b & 250 & 4.0 & 2.1 & Suboptimal & $104 \leq r \leq 304$ & 0 \\
Test 2c & 250 & 4.0 & 3.15 & Superoptimal & $104 \leq r \leq 304$ & 0 \\
Test 3 & 200 & 4.0 & 2.1 & Optimal & $104 \leq r \leq 304$ & 0 \\
Test 4 & 150 & 4.0 & 0.6 & Optimal & $104 \leq r \leq 304$ & 0 \\
Test 5 & 250 & 4.0 & 2.5 & Optimal & 154 & $-150 \leq z \leq 900$ \\
Test 6 & 250 & 4.0 & 2.5 & Optimal & 142 & $0 \leq z \leq 400$ \\
Test 7 & 250 & 4.0 & 2.5 & Optimal & 182 & $-100 \leq z \leq 500$ \\
Test 8 & 250 & 4.0 & 2.5 & Optimal & 261 & $-100 \leq z \leq 700$ \\
\hline
\end{tabular}

by the thrust stand recorded as an offset. A linear drift with time over the duration of the test was assumed and this drift was subtracted from the final data.

In all cases, the thruster was first operated at its most efficient magnetic field, as determined by adjusting the magnetic current while monitoring the efficiency calculated in real time by the thrust stand software. This optimization was performed with the cathode at $r=104 \mathrm{~mm}, z=0 \mathrm{~mm}$. In Test 1 and Test 2 , the $300 \mathrm{~V}$ and $250 \mathrm{~V}$ cases, the magnet current was adjusted above and below the optimal values. For Test 1, values $25 \%$ above and below the optimal were chosen. For Test 2 , where the optimal magnet current was $2.5 \mathrm{~A}$, values of $3.15 \mathrm{~A}$ and $2.1 \mathrm{~A}$ were chosen for the super- and sub-optimal currents because they matched magnet currents used in Test 1 and Test 3 , respectively.

Between Tests 5 and 6 the thruster was repositioned lower, closer to the thrust stand, to reduce thermal drifts in the thrust measurements. However, the cathode could no longer be positioned exactly level with the thrust center line as in the previous tests. The displacements listed in Table 7.1 have been corrected to account for this, and still show the absolute distance from cathode orifice center to thrust axis.

\subsection{Results}

The graphs in Figure 7.2 plot thrust, efficiency, anode current, and cathode coupling voltage as a function of cathode radial position for each of the radial tests. In all cases, when the cathode was near the thruster, particularly when it was as close as possible to the thruster at $r=104$, significant heat was exchanged between the cathode and the anode. This was evidenced by an increase in voltage in the current-limited supplies for the cathode heater and the magnet coils. This heat exchange made it difficult to achieve thermal equilibrium and the error on these data points is therefore significantly higher 
than on those where the cathode was positioned farther from the thruster. Note that these experiments were performed before the addition of the heat shield, as discussed in Section 4.4.4.

Given the thermal drifts in the thrust stand compounded with the error in the linear fit, the uncertainty in the thrust measurement is estimated at 5\%. Propagating the uncertainties of the thrust measurement and the mass flow rate through the efficiency equation (Equation 2.22), I estimated the uncertainty on the efficiency measurements at $9 \%$ of the stated values. ${ }^{*}$

Test 1 is at the nominal operating point for the thruster. For all magnet currents, the cathode coupling voltage trends upwards as the cathode is moved away from the thruster, changing by approximately $10 \mathrm{~V}$, or $3 \%$ of the discharge voltage. The anode current holds steady and the thrust increases, resulting in an efficiency increase of approximately $3 \%$ to $5 \%$.

During Test $1 \mathrm{c}$ it was necessary to restart the thruster. The numbered points in Figure 7.2c show the order in which the points were taken, and the asterisk denotes the first point after the restart. This is potentially important as the cathode coupling voltages did not return to exactly the same position after the restart.

Test 2, run at a discharge voltage of $250 \mathrm{~V}$, shows the most interesting behavior. All magnetic field conditions exhibited a peak in cathode coupling voltage between 40 and $164 \mathrm{~mm}$, followed by a trough between 184 and $204 \mathrm{~mm}$, and then a gradual rise as the cathode was moved out to $304 \mathrm{~mm}$. Again the discharge current held constant, while the thrust and efficiency tracked the changes in cathode coupling voltage.

Test 3, run at a discharge voltage of $200 \mathrm{~V}$, also exhibited anomalies due to thruster restarts. Again, the plot shows the order in which the data were taken, with asterisks denoting the first point after a thruster restart. Note that there is a significant jump in cathode coupling voltage after the restart between points five and six. In particular, the cathode coupling voltage dropped by approximately $2 \mathrm{~V}$ between points thirteen and five. It is likely that some of this drop is attributed to the change in conditions between thruster restarts. However, the magnitude of the change in cathode coupling voltages across the range of cathode positions is much smaller than in either Test 1 or Test 2.

Test 4 , at a discharge voltage of $150 \mathrm{~V}$, shows drastically different behavior than the previous tests. Here the cathode coupling voltage significantly decreased with increasing radial distance. The thrust and efficiency also decreased, somewhat in step with the cathode coupling voltage. Note also that the discharge current, in contrast to the other cases, changed drastically with cathode position.

Test 5 was performed at the optimal magnet current of $2.5 \mathrm{~A}$ and a discharge voltage of $250 \mathrm{~V}$. Here, the cathode was placed $154 \mathrm{~mm}$ away from the thrust axis, radially, and the cathode swept through axial positions. As the cathode was moved behind the

${ }^{*}$ For example, if the efficiency reported is $50 \%$, then the one-sigma range of the uncertainty is $0.5 \pm 0.5 \times 0.09$, that is, between $45.5 \%$ and $54.5 \%$ 
thruster poor performance was recorded, along with low cathode coupling voltages. As the cathode was brought downstream of the thruster, the performance of the thruster and cathode coupling voltage improved significantly, reaching a peak at $175 \mathrm{~mm}$ downstream of the exit plane. The anode current is seen to vary slightly, but consistently, with the other measurements. This change in current is small in comparison to Test 4, but certainly much more pronounced than the remainder of the radial sweeps.

Tests 6-8 were taken at the same operating conditions as Test 5, that is, optimal magnet current of $2.5 \mathrm{~A}$ and a discharge voltage of $250 \mathrm{~V}$. However, these data were taken on a different day, following small modifications to the setup which were made in a partially successful attempt to further alleviate thermal drifts in the thrust stand. These data show good agreement in trends with the data taken in Test 5, particularly comparing Test 6 and Test 5 which were taken at nearby radial positions. The most notable difference between Test 5 and 6 is that the current is about 5\% higher in Test 6 , and does not change appreciably in Test 6 as it does in Test 5. The higher anode currents cause lower overall efficiencies in Test 6 as compared to Test 5. The reason for the change in current is not clear. One possibility is that the cathode is coupling to grounded surfaces differently due to the change in setup. None-the-less, the fact that the overall trends remain suggest that fundamental changes in cathode-HET coupling are occurring as the cathode position is changed.

Comparing Tests 6,7 , and 8 one consistently notes increasing cathode coupling voltages with increasing $\mathrm{z}$, up to a maximal point. The maximum increases with increasing $\mathrm{r}$, occurring at $\sim 150 \mathrm{~mm}$ for $r=142 \mathrm{~mm}, \sim 200 \mathrm{~mm}$ for $r=182 \mathrm{~mm}$, and $\sim 300 \mathrm{~mm}$ for $r=261 \mathrm{~mm}$. The thrust and efficiencies, again, loosely follow this trend, perhaps lagging behind a little as the coupling voltages begin to fall off.

\subsection{Discussion}

As these data were preliminary, and similar trends will be seen again in Chapter 8, this discussion will be brief. The trend of increasing thrust, efficiency and cathode coupling voltage with increasing radial and axial position was certainly unexpected. Particularly given the increase in $V_{\mathrm{cg}}$ this suggests that cathode coupling is actually improved as the cathode is moved further away from the thruster, at least up to a point. This is in contradiction to work performed by Walker and Gallimore, ${ }^{1}$ in which the opposite trend was noted. However, Walker and Gallimore operated a different thruster in a different vacuum chamber and measured performance over a physically larger range of radial cathode positions, but with a correspondingly coarser resolution. This may be sufficient to explain the differences. However, at the present time the reason for the discrepancy is unclear. 


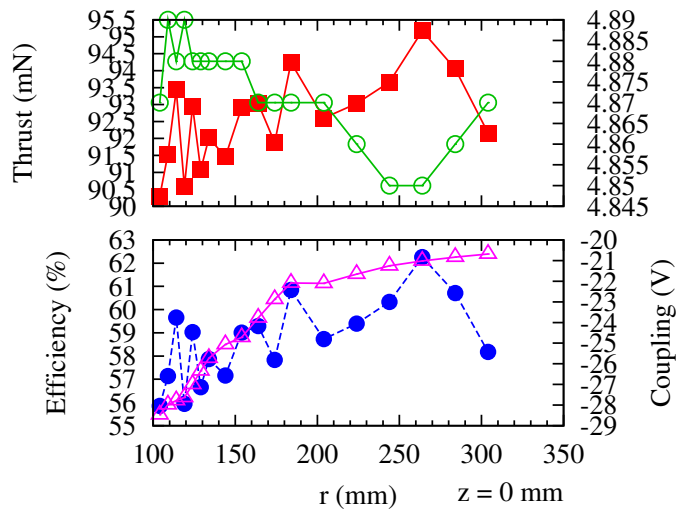

(a) Test 1a

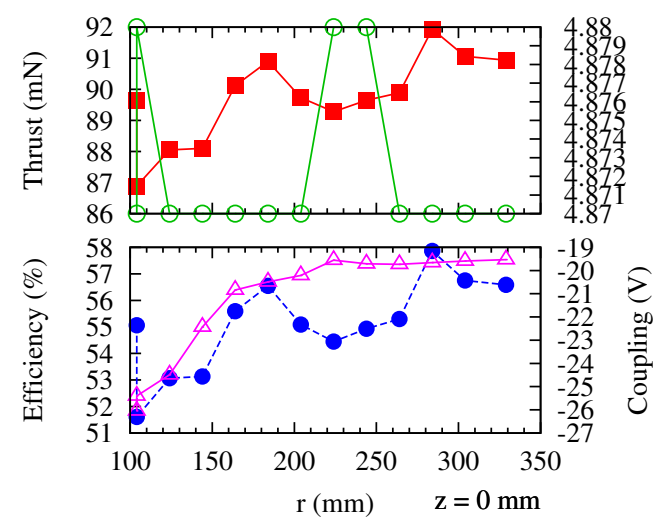

(b) Test $1 b$

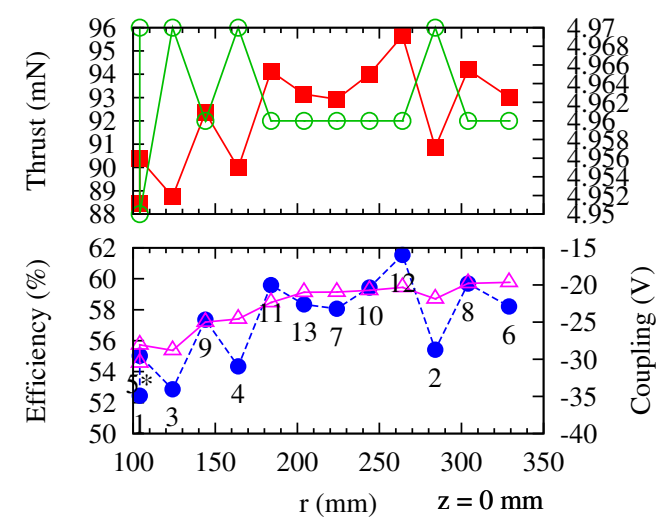

(c) Test $1 \mathrm{c}$

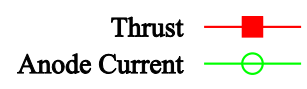

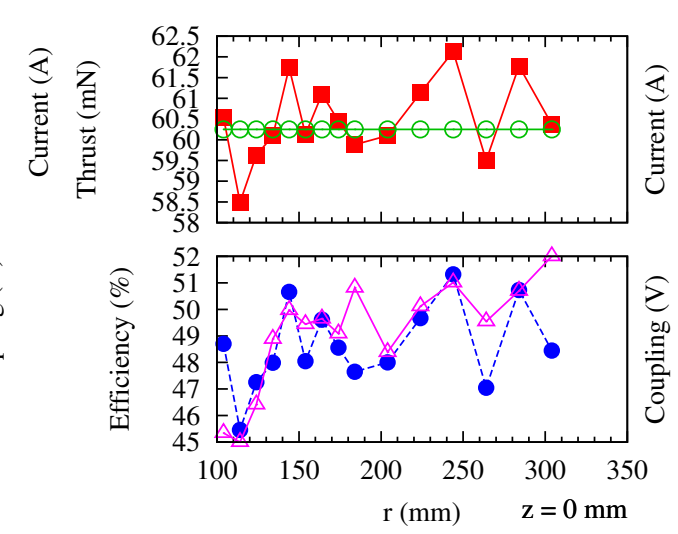

(d) Test $2 \mathrm{a}$

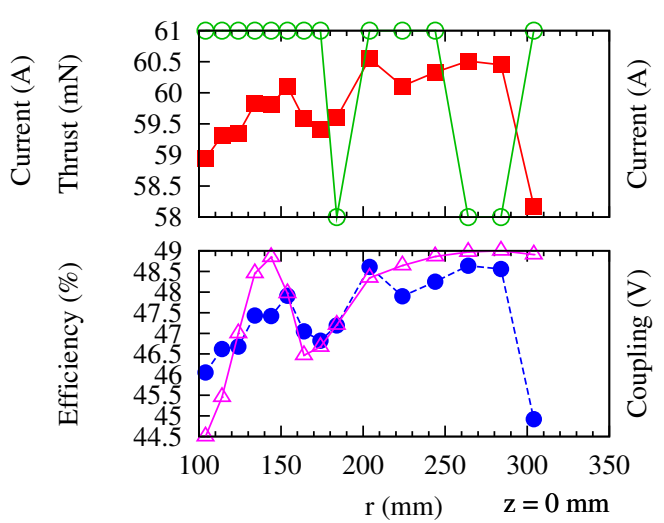

(e) Test $2 b$

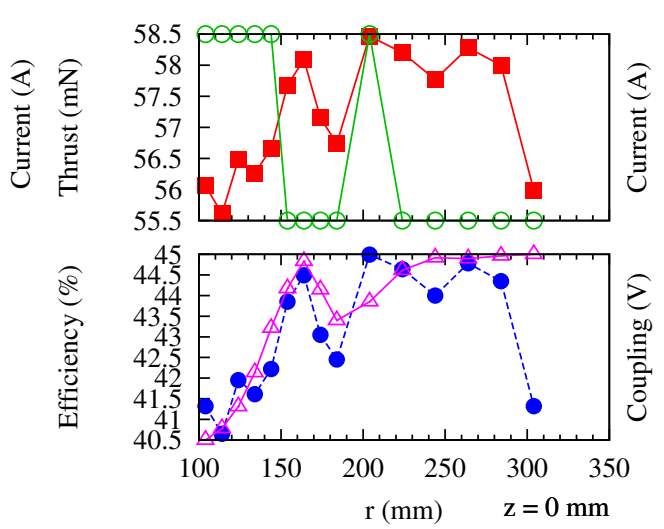

(f) Test $2 \mathrm{c}$

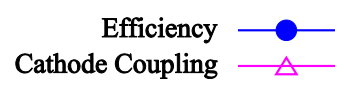

Figure 7.2: Results of the cathode position test 


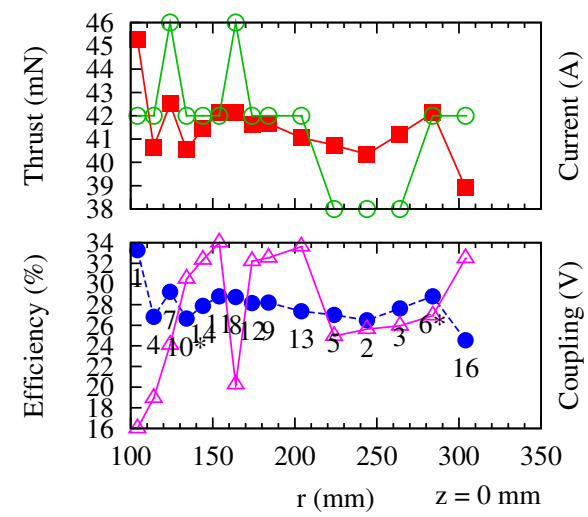

(g) Test 3

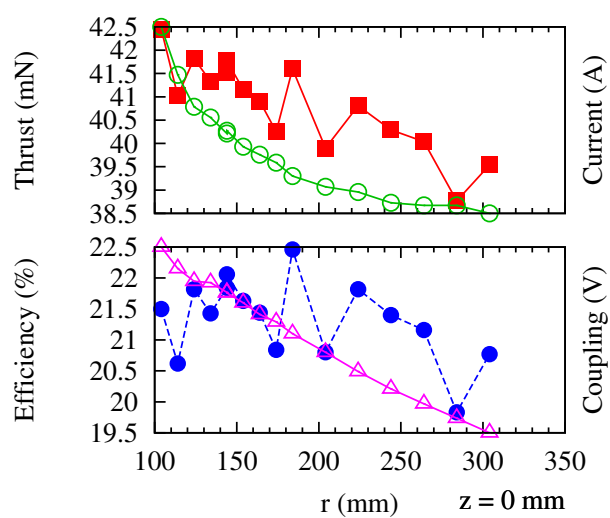

(h) Test 4

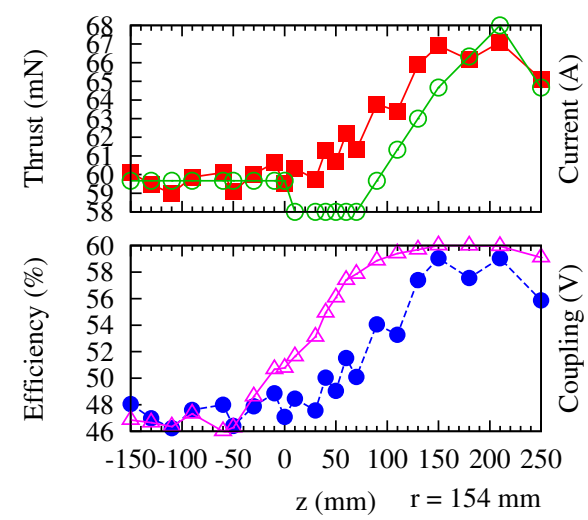

(i) Test 5

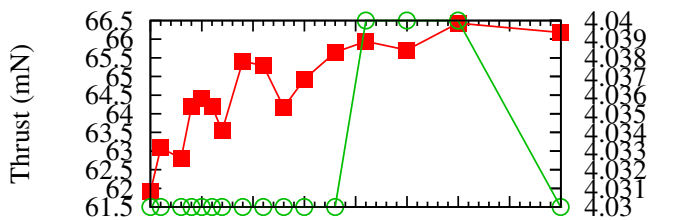

\section{这}

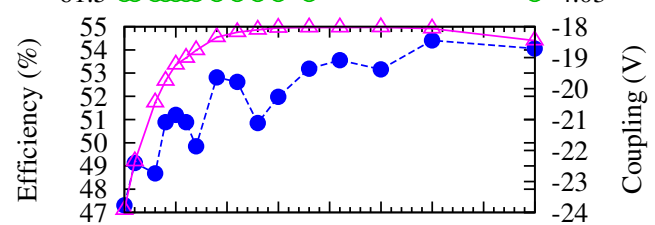

$0 \quad 50100150200250300350400$

$\mathrm{z}(\mathrm{mm}) \quad \mathrm{r}=142 \mathrm{~mm}$

(j) Test 6

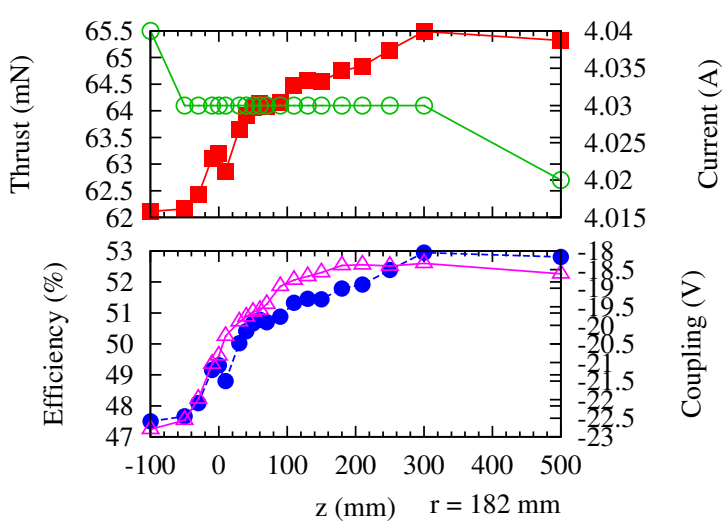

(k) Test 7
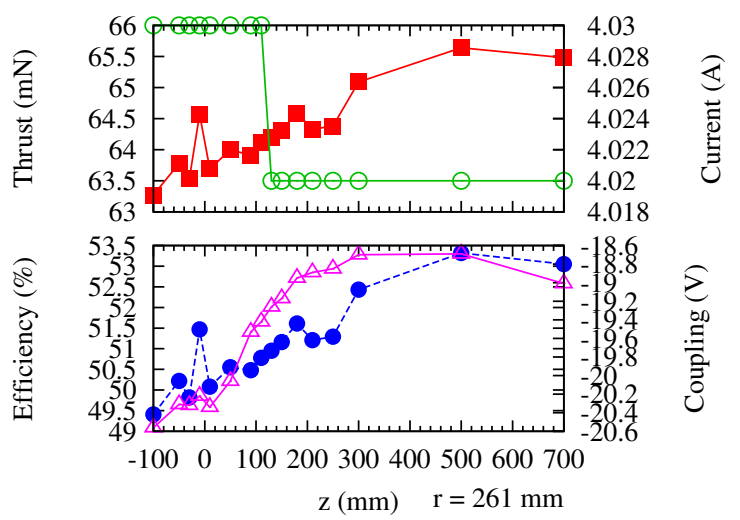

$\sum_{00}^{\circ}$
$\vdots$
0
0
0

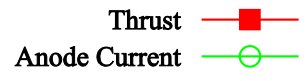

(l) Test 8

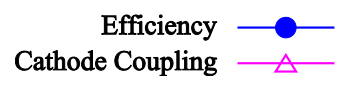

Figure 7.2: Results of the cathode position test (continued) 


\subsection{Performance vs. B Field}

Comparing the performance data from Section 7.2 with the magnetic field model presented in Chapter 6 proves interesting. Figure 7.3 overlays the field lines generated by the model on the performance results from Tests 6-8. In these figures, the color mapped data represent the performance quantity (thrust, efficiency, etc.), while the solid lines show the magnetic field lines as modeled in Chapter 6. The dotted contours show magnetic field strength in millitesla. Note that these data have only 3 radial positions, and the contours represent a considerable amount of interpolation. Regardless, one sees that thrust, efficiency, and $V_{\mathrm{cg}}$ as a function of cathode position tend to follow the magnetic field lines, while $I_{d}$ remains largely independent of cathode position. As the cathode moves into regions where the magnetic field lines intersecting the cathode closely approach the discharge channel the thrust and cathode coupling increase while discharge current remains constant. These changes result in efficiency improvements following the same trend with cathode position as thrust and $V_{\mathrm{cg}}$.

This comparison suggests that performance improves when the cathode is placed such that emitted electrons are near lines which carry them closer to the discharge channel. These field lines also bring the electrons to regions of higher neutral densities which result in lower Hall parameters, as seen in Figure 6.8. The increased electronneutral collisions enable the electrons to more easily cross the field lines in these regions. 
This page intentionally left blank 


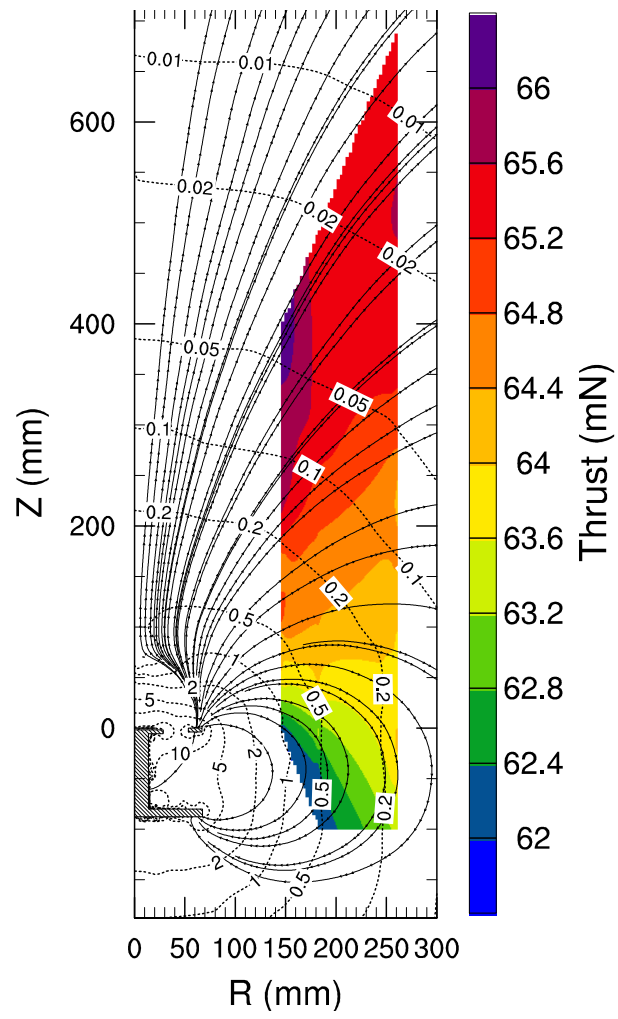

(a) Thrust

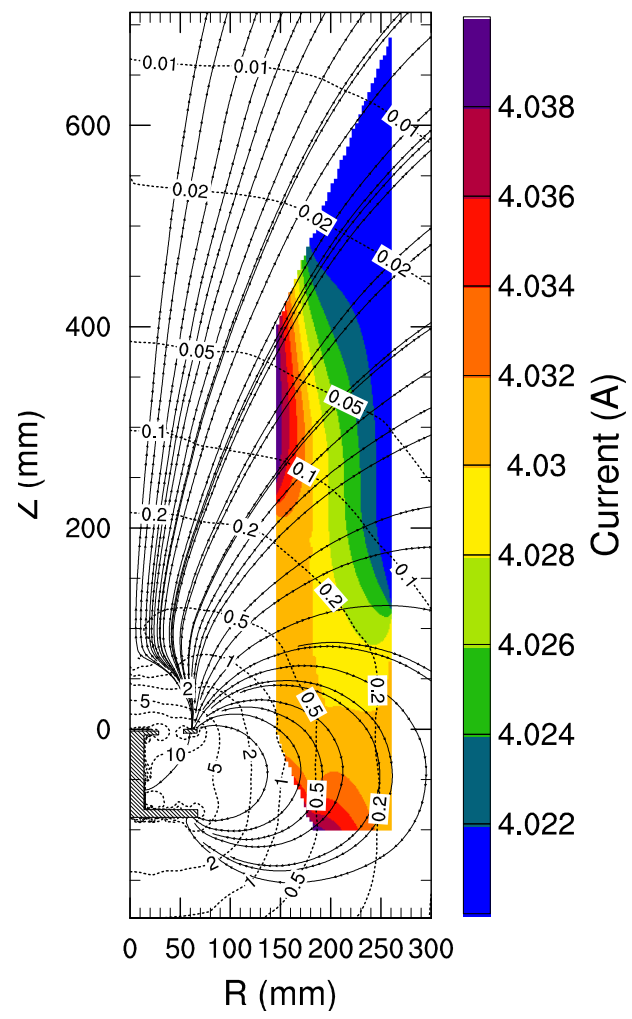

(b) $I_{d}$

Figure 7.3: Performance data overlaid with magnetic field lines. 

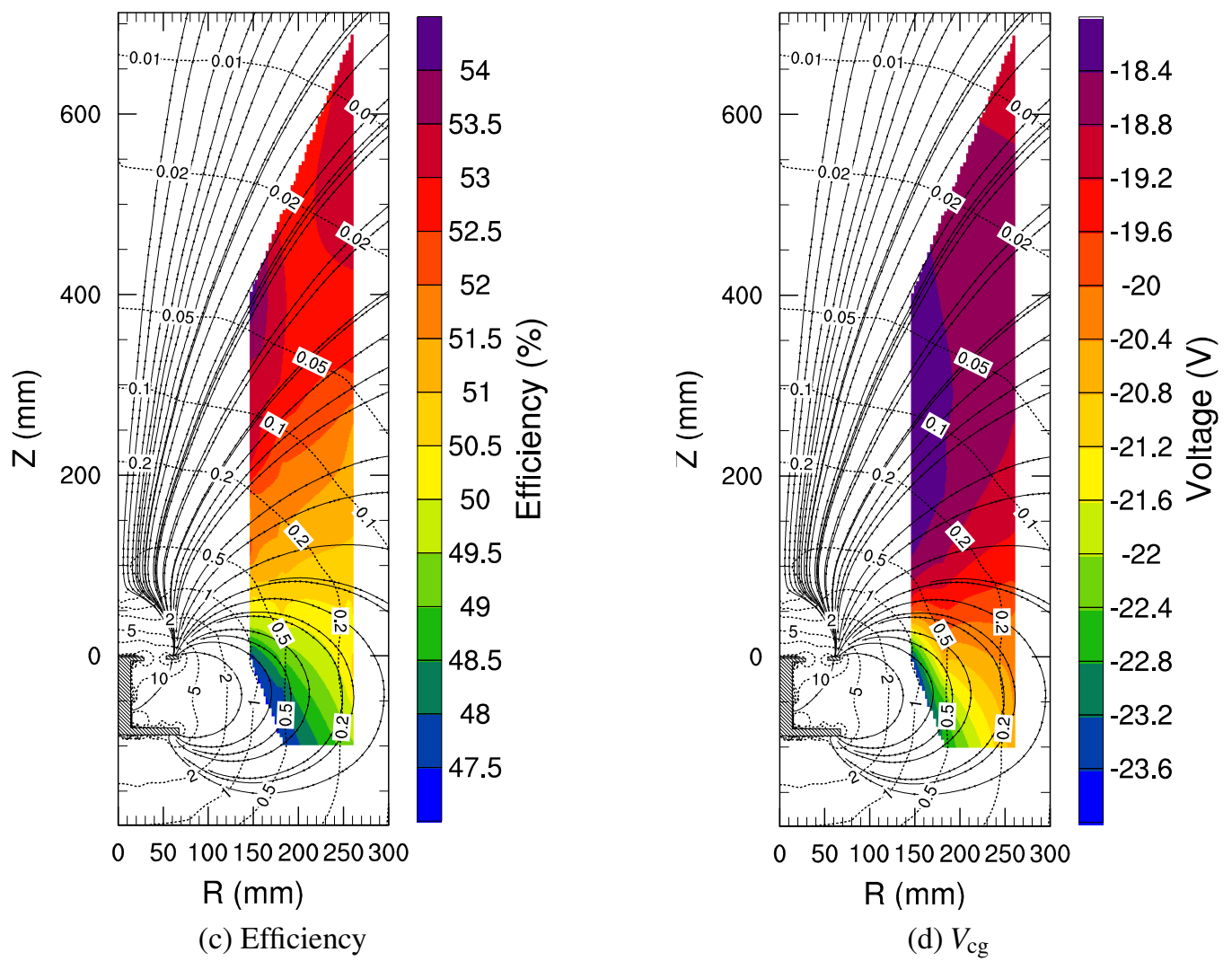

Figure 7.3: Performance data overlaid with magnetic field lines (continued). 


\section{References for Chapter 7}

[1] Walker, M. L. R. and Gallimore, A. D., "Hall Thruster Cluster Operation with a Shared Cathode," Journal of Propulsion and Power, Vol. 23, No. 3, May 2007, pp. 528-536. 


\section{Chapter 8}

\section{Separatrix Crossing Experiments}

\subsection{Motivation and Overview}

The correlation between the magnetic field and the performance data that was seen in the preliminary experiment suggested that magnetic fields are important in the coupling between the cathode and the thruster. This is not surprising, considering the effect that the magnetic field has on the motions of plasma electrons, as discussed in Chapter 2. Indeed, it has been suggested before, as reviewed in Chapter 3. Figure 6.6 is repeated as Figure 8.1. Note that the field lines inside of the separatrix surface will tend to trap electrons in front of the anode and in the beam. Meanwhile, field lines outside of the separatrix will tend to direct electrons either to the anode face, or, away from the beam altogether. Combined with the correlation between magnetic field and performance, this suggests that the separatrix surface is an important feature, and that a cathode would ideally be located inside of it, thereby introducing the electrons into a region that will trap them in the beam, rather than in a region that will keep them away.

The hypothesis of ideal cathode coupling within the separatrix was impossible to test with the setup of the preliminary experiment. Because the cathode was aligned with its axis parallel to the thruster it was impossible to place the cathode inside of the separatrix surface since the cathode would collide with the thruster. Therefore, the experiments discussed in this chapter were conducted with the cathode mounted at a 90 degree angle to the thrust axis. This allowed the cathode to be moved in a radial direction and to cross the separatrix.

In each run, the cathode was placed in a range of radial positions and at a fixed axial position. As in the preliminary experiment, radial positions were selected in random order to eliminate the possibility of time-dependent phenomena, such as thermal changes, from being misinterpreted as changes due to cathode position. At each position the cathode was run at three mass flow rates. At each condition, thrust, efficiency, and cathode coupling voltage were measured, as in the preliminary experiment. 


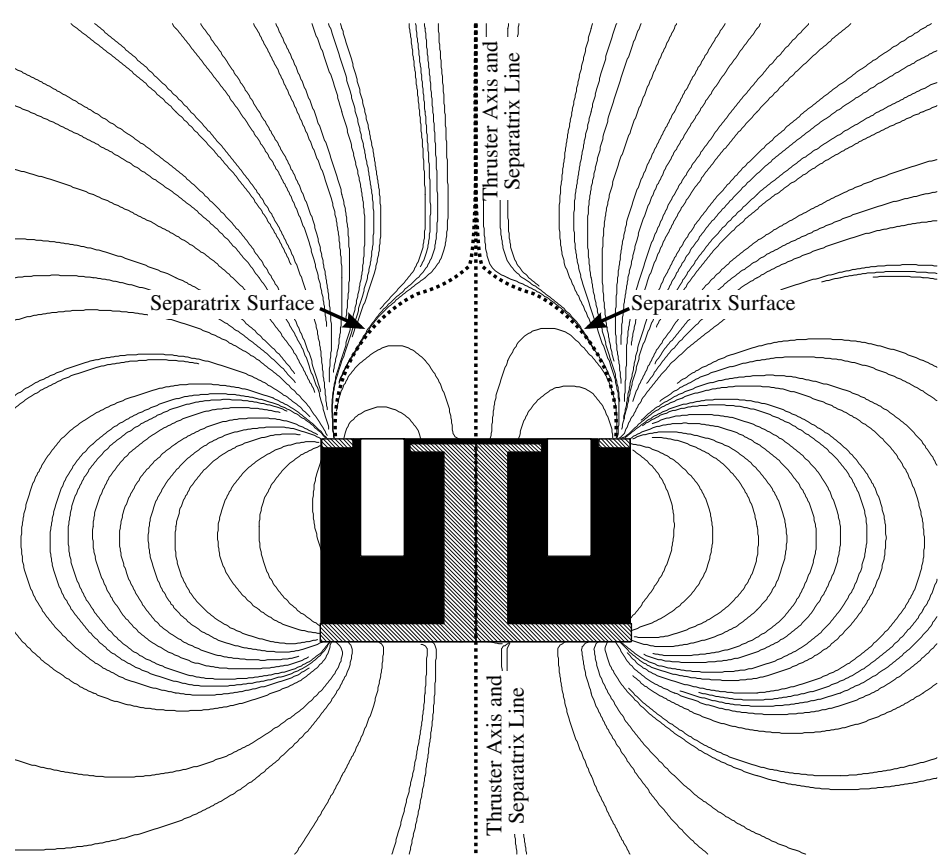

Figure 8.1: Magnetic field lines and separatrices for a typical HET.

This experiment also delves deeper into the mechanisms behind the changes in efficiency and the cathode coupling processes. At each operating condition the ion current density and the ion energy distribution function were measured with a Faraday probe and an RPA, respectively. From these, the efficiency can be broken down into various loss components. In an effort to understand how the efficiency components were being affected, near-field plasma properties were also measured at each operational condition with a double Langmuir probe. These data also provide some insight into the cathode coupling processes.

As will be shown, the optimal position for the cathode is indeed inside of the separatrix. Unfortunately, on small thrusters like the BHT-2000, it is not possible to cross the separatrix without placing the cathode very near or inside of the ion beam. These cathode locations are not practical for flight thrusters, as they would result in high sputtering of the cathode orifice and early cathode failure. On the BHT-2000, for a cathode mounted at 90 degree to the thrust axis with its center line at an axial distance of $z=30 \mathrm{~mm}$ downstream of the face, the separatrix occurs at a position $r=60 \mathrm{~mm}$. However, the outer edge of the discharge chamber has a radius of $50 \mathrm{~mm}$. Thus, even placing the cathode exactly at the separatrix, one has still placed the cathode uncomfortably close to the ion beam. (In fact, given the divergence of the beam, it is likely that this position is still heavily bombarded by ions.) To actually cross the separatrix, one must move the cathode in even farther. Since the goal of these experiments is not to pro- 
duce a flight thruster, but to understand the coupling, the cathode was, never-the-less, moved across the separatrix and into the beam.

Aware of the sputtering problems associated with moving the cathode inside the separatrix, I modified the thruster to move the separatrix out rather than moving the cathode in. This allowed the cathode to be placed within the separatrix, yet at a safer distance from the discharge channel. The modification will be discussed in Section 8.2.1. Having modified the thruster, I repeated the experiment at similar operating conditions as previously used with the unmodified thruster. The results of both unmodified and modified thrusters will be presented side-by-side through this chapter for comparison.

The original intent was to run this experiment using xenon as in the preliminary experiments. However, my supply of xenon was depleted after acquiring a complete set of data with the unmodified outer pole. Due to the volatile and extremely high price of xenon in mid-2008, the laboratory was unable to acquire more. Therefore, this experiment, as well as the experiment in Chapter 9 were performed with cheaper, but less efficient, krypton. The data from the xenon half-experiment is presented with minimal analysis in Appendix D. An efficiency lower by 5\%-15\% was expected when running with krypton. 1,2 However, these data show a 25\%-35\% lower efficiency than when running with xenon. It is not clear why such unexpectedly low efficiencies occurred, as the only thing changed between the xenon run and the krypton run was the propellent.

\subsection{Design of Experiment}

\subsubsection{Design of the extended outer pole}

To move the separatrix farther from the discharge channel, I designed an extended outer pole piece in which the length of each side was extended by $70 \mathrm{~mm}$. This corresponded with the maximum sized plate of ASTM-A848 magnet iron that could be readily purchased. No other dimensions of the original pole piece were modified. Figures $8.2 \mathrm{a}$ and $8.2 \mathrm{~b}$ show drawings of the pole pieces and photographs of the pieces mounted to the thruster. With the Extended Outer Pole (EOP), the field of the thruster is modified such that at $z=30 \mathrm{~mm}$ the separatrix occurs at $78 \mathrm{~mm}, 18 \mathrm{~mm}$ further than with the Original Outer Pole (OOP), as shown in Figure 8.2c.

An unfortunate side effect of the extended outer pole is that it not only modifies the external magnetic field, but it also modifies the internal magnetic field. In Figure 8.2c this is most evident near the inner edge of the outer pole piece, where the shape of the field strength contours have changed. This problem will be discussed later in Section 9.3. 

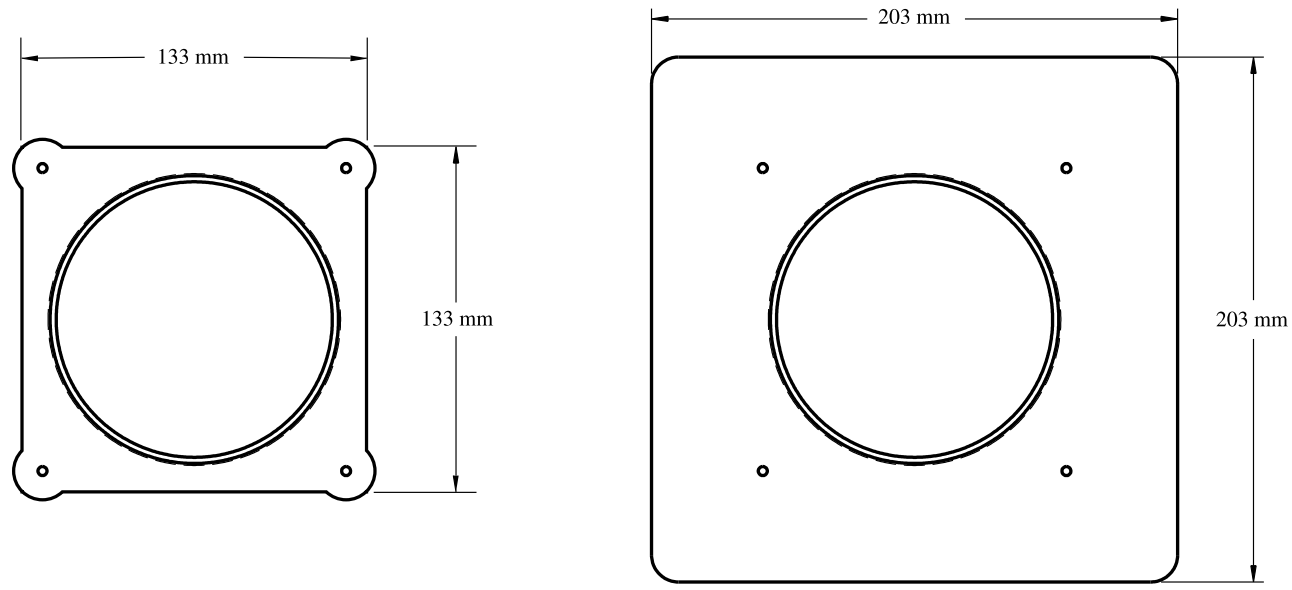

(a) Drawings of the original and the extended outer pole pieces
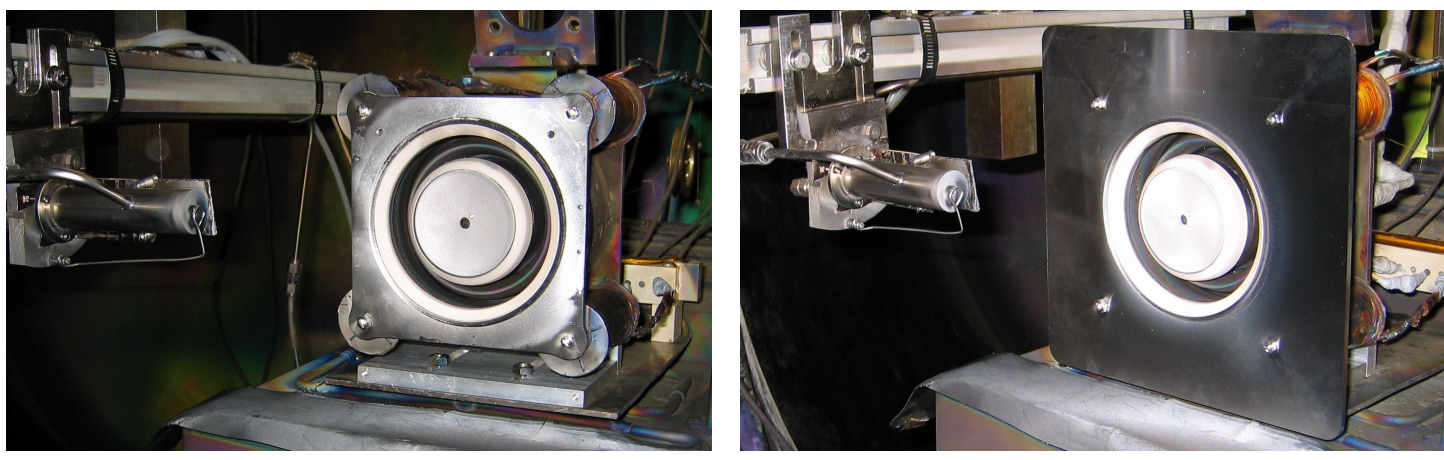

(b) Photographs of the thruster with the original and the extended outer pole
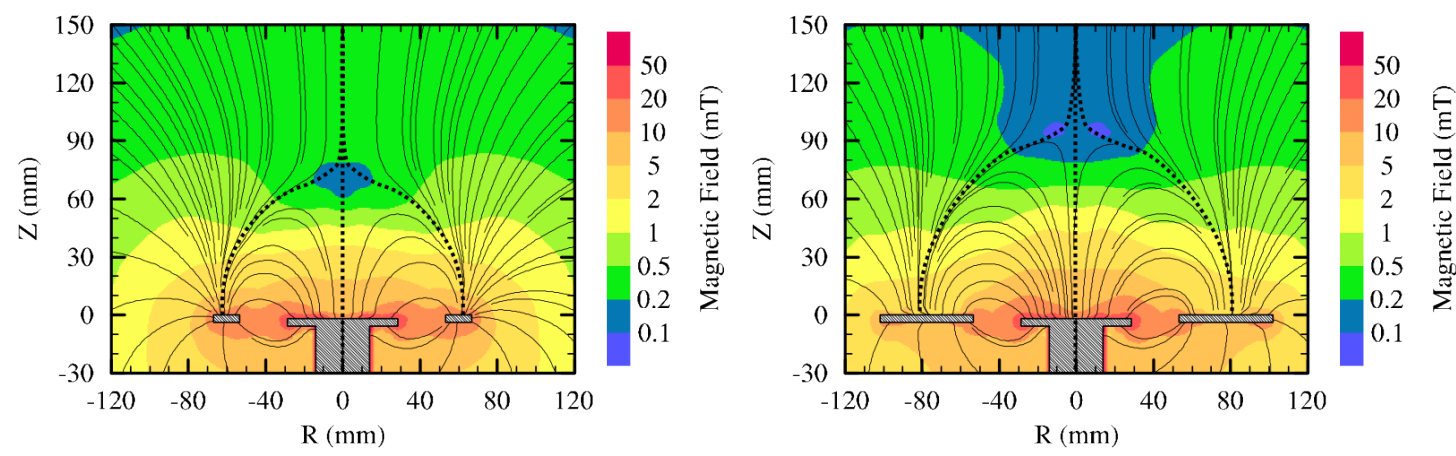

(c) Magnetic field of the thruster with the original and the extended outer poles

Figure 8.2: Design and effect of the extended outer pole 


\subsubsection{Setup}

A general overview of the experimental setup can be found in Section 4.2. As always, the HET was mounted on a thrust stand. On the first run, the unmodified thruster was used, while on the second, the thruster modified with the extended outer pole was used. The cathode was mounted on a linear motion stage and could be moved from $40 \mathrm{~mm}$ to $250 \mathrm{~mm}$ radially away from the thrust axis, at a fixed axial distance of $30 \mathrm{~mm}$ from the thruster exit plane (see Figures 4.1 and 4.2). It was mounted at a $90^{\circ}$ angle between its axis and the thrust axis. A Farday probe and a retarding potential analyzer (RPA) were placed on a boom mounted to a rotational stage directly above the intersection of the thrust axis and the exit plane. The probes were mounted at a distance of $250 \mathrm{~mm}$. A double probe was mounted on a two-axis motion table capable of positioning the probe so that it could interrogate the near field plume of the thruster, from $z=5$ to $105 \mathrm{~mm}$ and from $r=-180$ to $+180 \mathrm{~mm}$.

\subsubsection{Procedure}

The general experimental procedure can be found in Section 4.3. The aspects specific to this experiment follow. In each experiment, the thruster was set at an operating voltage of $250 \mathrm{~V}$, chosen to match the preliminary experiments. Due to a global supply shortage of xenon, krypton was used as the propellent. The preliminary experiments were run at $4 \mathrm{mg} / \mathrm{s}$ of xenon, which is $41 \mathrm{SCCM}$. These experiments were run with the same volumetric flow rate, and therefore number flow rate. This corresponds to a mass flow rate of $2.553 \mathrm{mg} / \mathrm{s}$ of krypton.

For the original outer pole, a magnet current value of $2.5 \mathrm{~A}$ was chosen to match prior values. In these experiments, $2.5 \mathrm{~A}$ was slightly above the optimal point, but more importance was placed on matching prior magnetic field strengths. With the extended outer pole, it was impossible to run the thruster at $2.5 \mathrm{~A}$. Therefore, the optimal value of 1.5 A was chosen. All magnet current optimization was performed with the cathode located at $z=100 \mathrm{~mm}$.

After the thruster stabilized, thrust, discharge current, and efficiency of the thruster were measured over a period of 90-120 s. After recording the performance, the cathode was moved to a new location, randomly chosen from the 14 positions: from $40 \mathrm{~mm}$ to $100 \mathrm{~mm}$ in $10 \mathrm{~mm}$ intervals, from $100 \mathrm{~mm}$ to $200 \mathrm{~mm}$ in $20 \mathrm{~mm}$ intervals, and $250 \mathrm{~mm}$.

After all of the performance data were taken at a given cathode mass flow, RPA and Faraday probe sweeps were performed at five cathode positions: $40 \mathrm{~mm}, 60 \mathrm{~mm}$, $80 \mathrm{~mm}, 120 \mathrm{~mm}$, and $200 \mathrm{~mm}$. In the $\dot{m}_{c}=10$ SCCM case, the $120 \mathrm{~mm}$ position was not acquired. Both probes were mounted at a constant radial distance of $250 \mathrm{~mm}$ from the center of the thruster exit plane. The Faraday probe was swept from -90 to 60 degrees in 2.3 degree increments. At each increment, 1000 samples were taken at a rate of $10 \mathrm{kHz}$ and averaged. The RPA was swept in 10 degree increments over the same range. At 
each increment, 5 sweeps from 0 to $300 \mathrm{~V}$ were taken with either 300 or 600 points per sweep.

Finally, the double probe data were acquired. The double probe scans were designed to minimize heating of the probe. The probe was swept as fast as the Keithley 2410 used to drive them would allow: approximately $100 \mathrm{~Hz}$. Each sweep consisted of 100 points from -40 to $40 \mathrm{~V}$ applied between the two probes.

After all performance and probe data were acquired at each of the cathode positions, the cathode mass flow rate was adjusted and the experiment repeated. Cathode mass flow rates of 10 SCCM, 5 SCCM, and 2 SCCM were chosen. Ten SCCM is the nominal cathode ignition flow rate, and 2 SCCM was the lowest cathode mass flow at which the cathode would stably operate.

\subsection{Performance}

\subsubsection{Results}

The thrust, discharge current, total and anode efficiencies, and cathode coupling voltage are plotted as a function of cathode position for each pole piece and at each of the three cathode mass flow rates in Figure 8.3. The $r$ location of the separatrix at $z=30 \mathrm{~mm}$ is plotted with a dashed, vertical line for each pole piece. Error bars are calculated estimates of the uncertainty in the measurements, as discussed below.

The uncertainty in the thrust measurements is estimated at $2 \mathrm{mN}$ based on observations of the remaining drifts in the thrust stand. The uncertainty in the efficiencies are calculated according to the standard method. For a function $f(a, b)$, the variance- the square of the uncertainty-is given by

$$
\sigma_{f}^{2}=\left(\frac{\partial f}{\partial a}\right)^{2} \sigma_{a}^{2}+\left(\frac{\partial f}{\partial b}\right)^{2} \sigma_{b}^{2}
$$

Recall the equation for thrust efficiency,

$$
\eta=\frac{T^{2}}{2 \dot{m} I_{d} V_{d}} .
$$

Since the uncertainties in $I_{d}$ and $V_{d}$ are negligible, variance in the efficiency is given by

$$
\begin{aligned}
\sigma_{\eta}^{2} & =\left(\frac{T}{\dot{m} P}\right)^{2} \sigma_{T}^{2}+\left(\frac{T^{2}}{2 \dot{m}^{2} P}\right)^{2} \sigma_{\dot{m}}^{2} \\
& =\eta^{2}\left(\frac{4 \sigma_{T}^{2}}{T^{2}}+\frac{\sigma_{\dot{m}}^{2}}{\dot{m}^{2}}\right) .
\end{aligned}
$$




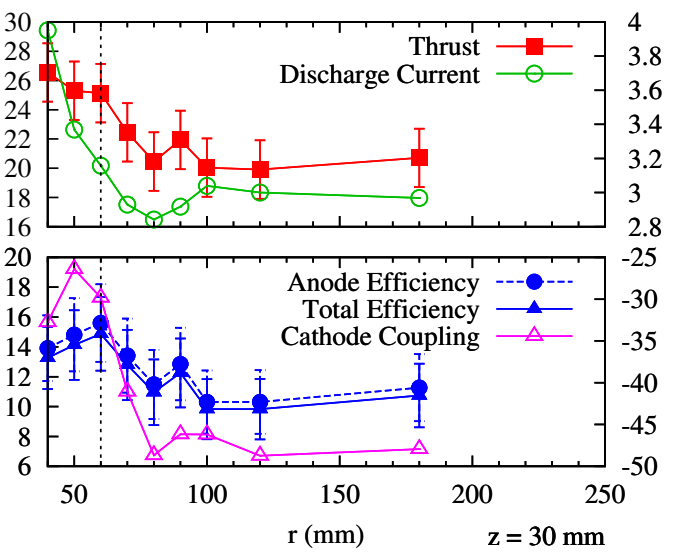

(a) OOP, $\dot{m}_{c}=2 \mathrm{SCCM}$

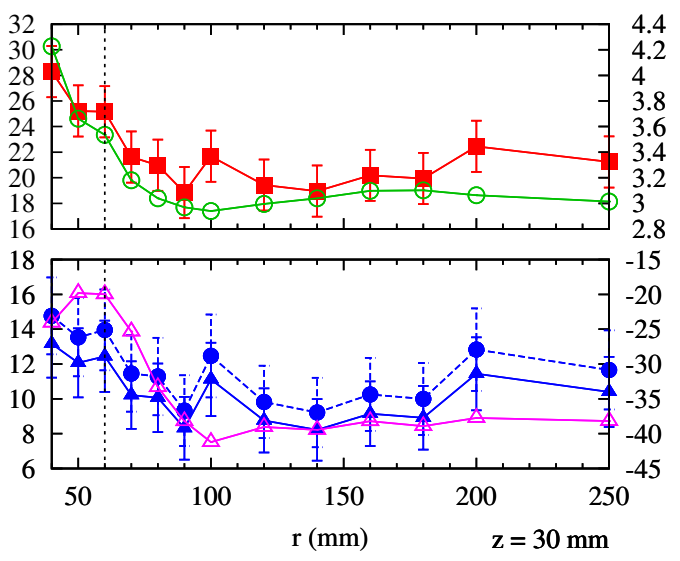

(c) OOP, $\dot{m}_{c}=5 \mathrm{SCCM}$

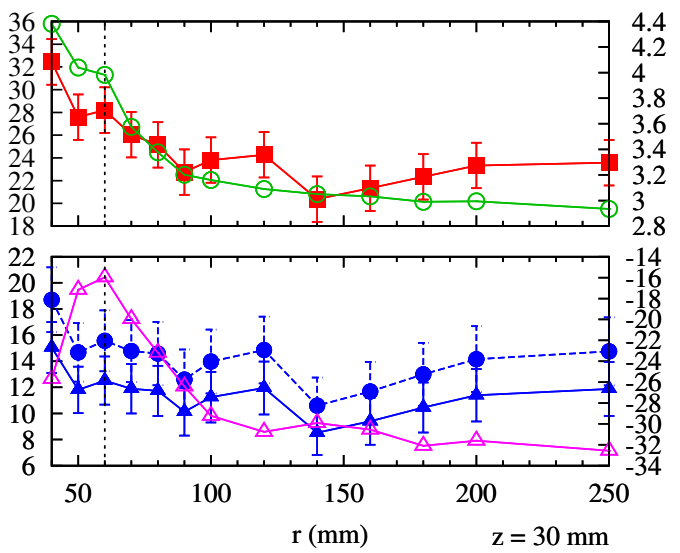

(e) OOP, $\dot{m}_{c}=10 \mathrm{SCCM}$

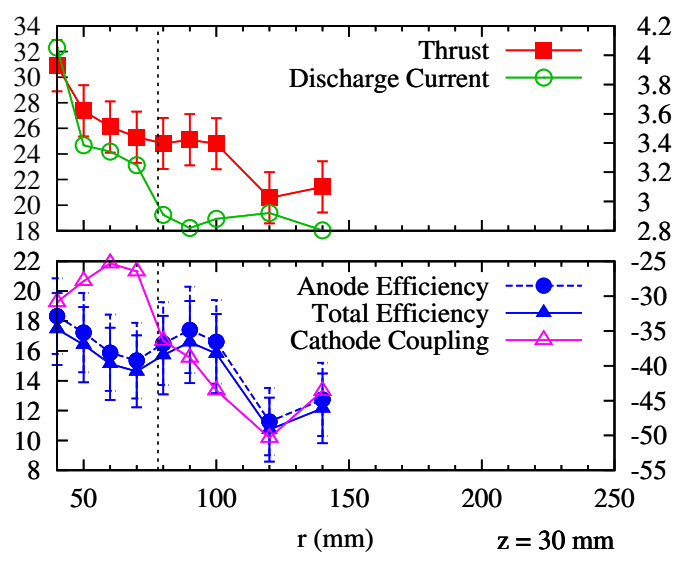

(b) EOP, $\dot{m}_{c}=2 \mathrm{SCCM}$

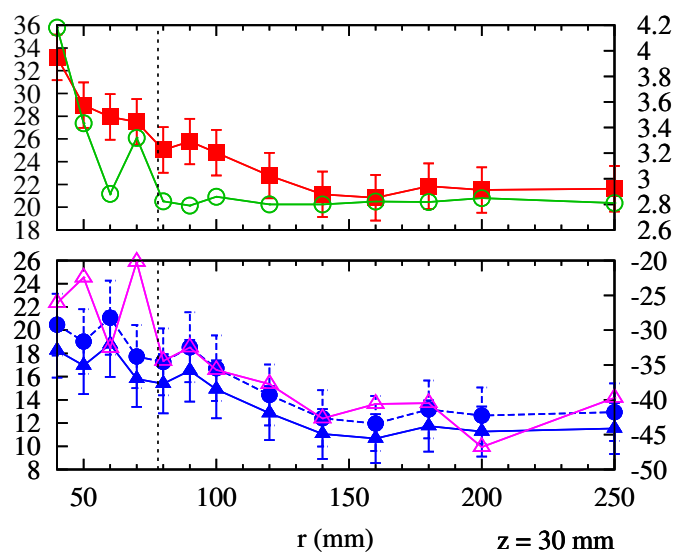

(d) EOP, $\dot{m}_{c}=5 \mathrm{SCCM}$

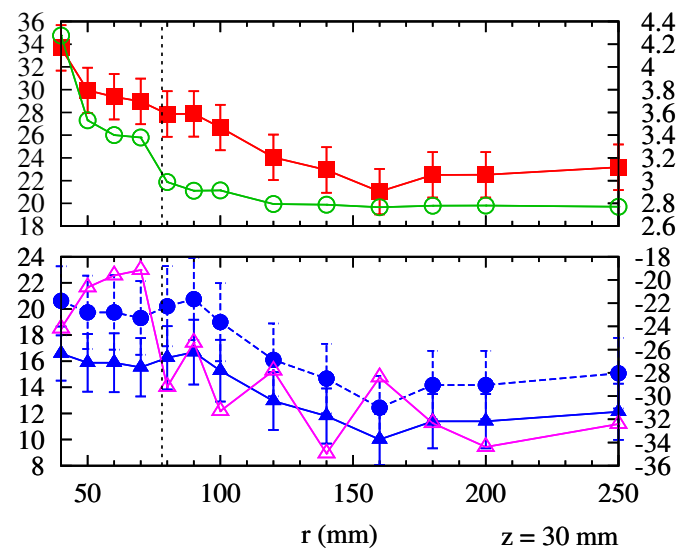

(f) EOP, $\dot{m}_{c}=10 \mathrm{SCCM}$

Figure 8.3: Thruster performance as a function of cathode position while operating on krypton. 
Therefore the uncertainty is given by

$$
\sigma_{\eta}=\eta \sqrt{\frac{4 \sigma_{T}^{2}}{T^{2}}+\frac{\sigma_{\dot{m}}^{2}}{\dot{m}^{2}}} .
$$

For anode efficiencies, we have $\dot{m}=\dot{m}_{a}$ and Equation 8.4 may be used directly. For total efficiencies we must substitute $\dot{m}=\dot{m}_{a}+\dot{m}_{c}$. The variance of this quantity is given by

$$
\sigma_{\dot{m}}^{2}=\sigma_{\dot{m}_{a}}^{2}+\sigma_{\dot{m}_{c}}^{2}
$$

The uncertainty in the mass flow is $1 \%$ of the mass flow controller's full scale range. This yields 2 SCCM for the anode mass flow controller, and 0.2 SCCM for the cathode flow controller.

\subsubsection{Discussion}

Several trends are apparent in the performance data shown in Figure 8.3. First of all, similar trends are seen with the OOP as seen in the preliminary experiments between $100 \mathrm{~mm}$ and $250 \mathrm{~mm}$. In this range, the thrust remains relatively constant, with a slight increase as the cathode is moved out beyond $140 \mathrm{~mm}$. Also in this range, the discharge current remains relatively constant, as does the cathode coupling voltage in most cases. These trends are quite similar to the trends seen in the preliminary experiment. (They are also similar to the trends seen in the xenon half-experiment in Appendix D.) The increase in efficiency from $100 \mathrm{~mm}$ and $250 \mathrm{~mm}$ reflects the increases seen in Section 7.2. Trends with the EOP look slightly different, with the increase in thrust occurring further out, closer to $160 \mathrm{~mm}$. The increase is also of smaller magnitude. It is interesting to note that the difference in the position of the inflection point is about the same as the $18 \mathrm{~mm}$ difference between the separatrices of the OOP and the EOP.

The area inside of $100 \mathrm{~mm}$ was inaccessible in the preliminary experiments. Inside this area, there are consistent trends of increasing thrust and discharge current. The efficiency generally increases, though in some cases it may reach a plateau inside of the separatrix. The cathode coupling voltage shows a particularly interesting trend; as $r$ is decreased, the coupling voltage increases until the cathode crosses the separatrix, at which point it begins a smaller decrease.

The increasing discharge current with decreasing $r$ is likely due, in part, to increased ingestion of cathode propellent. The greater increases associated with cathode mass flow rates attest to this. However, the increase is too large to be totally accounted for by this effect. If we assume that every cathode neutral is ingested and becomes singly-ionized we can calculate the maximum amount of current that could be added by propellent ingestion according to: 


$$
\begin{gathered}
\dot{N}_{c}=\frac{\dot{m}_{c}(\mathrm{SCCM}) \cdot 1 \times 10^{-6}\left(\mathrm{~m}^{3} / \mathrm{cc}\right) \cdot 101325 \mathrm{~Pa}}{k \cdot 273 \mathrm{~K} \cdot 60(\mathrm{~s} / \mathrm{min})} \\
I_{\text {ingested }}=e \dot{N}_{c} .
\end{gathered}
$$

This yields a maximum possible increase in discharge current of $0.14 \mathrm{~A}, 0.36 \mathrm{~A}$ and $0.72 \mathrm{~A}$ for the $\dot{m}_{c}=2,5$ and $10 \mathrm{SCCM}$, respectively. Looking at Figure 8.3, one sees that for every cathode flow rate for both the EOP and the OOP the jump in discharge current between when the cathode is placed at its farthest radial distance and at its closest is at least $1 \mathrm{~A}$. Clearly this jump in current cannot be explained by ingested propellent alone.

In most cases, particularly with the EOP, a large jump in discharge current is associated with crossing the separatrix. It is likely that by crossing the separatrix, the thruster entered a different mode of operation, which lead to increased recycle current. Despite this increase in current, the overall efficiency of the thruster was not significantly affected. The increase in thrust partially offsets in the increase in discharge current in Equation 2.20.

A simultaneous increase in discharge current and coupling voltage, such as that seen when the cathode is moved into the separatrix, suggests a decrease in the effective resistance between the cathode and the beam. Recall that all of the current in the beam and the recycle current to the anode originate from the cathode. A higher coupling voltage represents a smaller potential difference between the cathode and the beam. (This will be further shown in the cathode plume data, in Section 8.6.) According to Ohm's law, $V=I R$, if the current goes up and the potential difference goes down, necessarily the resistance goes down. This suggests that moving the cathode inward, particularly across the separatrix, results in a reduction of plasma impedance. This reduces the amount of work required to move the electrons from the cathode to the positions required to maintain charge neutrality and complete the electrical circuit.

\subsection{Ion Beam Properties}

\subsubsection{Ion current density profiles}

The ion current density as a function of angle is plotted in Figures 8.4 and 8.5. The data are plotted on a polar graph. However, note there is a change of units at $r=$ $250 \mathrm{~mm}$. Inside this region, a representation of the thruster and cathode are plotted for reference. Outside, the radial dimension corresponds to current density. Each subfigure groups together the data taken at the same cathode mass flow but different cathode positions.

One notes an increased broadening and decreased center-spike magnitude as the cathode is moved away from the thrust axis. There is also a decrease in the overall 
magnitude of the current densities with decreasing cathode mass flow, though this is less pronounced when the cathode is further from the thrust axis. The broadening of the beam can be expected to result in decreased performance as beam divergence increases. This will be discussed further in Section 8.5.

\subsubsection{Ion energy distributions}

Figures 8.6 through 8.11 show the IEDFs as a function of angle for each outer pole, cathode position and mass flow operating point measured. The IEDFs were measured with an RPA as discussed in Section 5.3. Each figure shows the probability of an ion at a given angle having an energy that corresponds to the radial dimension. To calculate the total probability of finding an angle at a specific angle and energy, one would need to multiply the probability on the IEDF by the normalized current density at that angle.

The data were taken at 10-degree intervals from -90 degrees to 60 degrees. Missing data is due to poor signal-to-noise ratio at that location. The data have been interpolated in the figures. The corresponding current density as measured by the Faraday probe is overlaid. Note that the arrangement of the radial axis is identical to that of Figure 8.4.

The most striking feature of these graphs is that as the cathode is moved away from the thruster, the distributions become narrower in energy space. Furthermore, the broad energy distribution at 0 degrees seems to go away as the cathode is moved away from the thrust axis and as cathode mass flow is decreased. When present, the narrow spike in probability density occurs at approximately $200 \mathrm{~V}$, regardless of cathode position, and extends out to about -60 degrees. It appears to vary slightly with cathode mass flow, moving from $\sim 200 \mathrm{~V}$ to $\sim 210 \mathrm{~V}$ as the mass flow is varied from 2 SCCM to 10 SCCM.

Finally, the data often show a low energy spike near -90 degrees. This is particularly true of the xenon data presented in Appendix D.3. However, signal levels here are quite small, and I have low confidence in the data taken between -80 and -90 degrees. 
This page intentionally left blank 


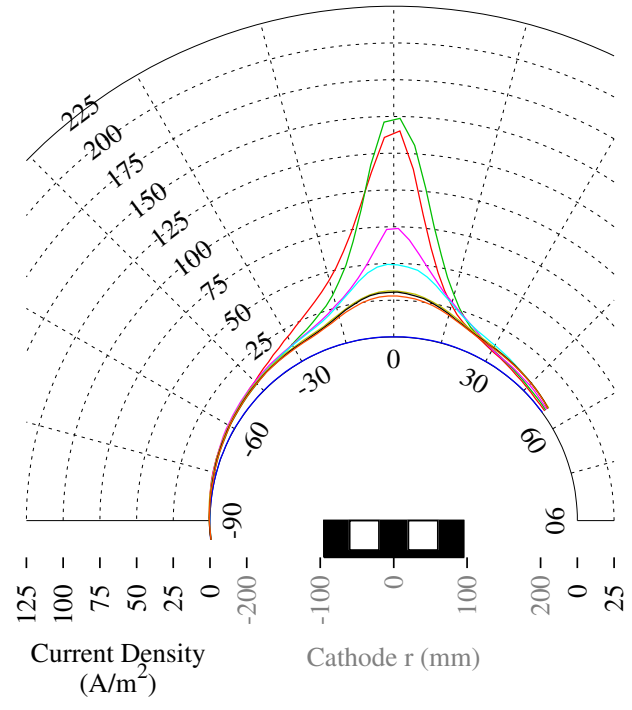

(a) $\dot{m}_{c}=2 \mathrm{SCCM}$

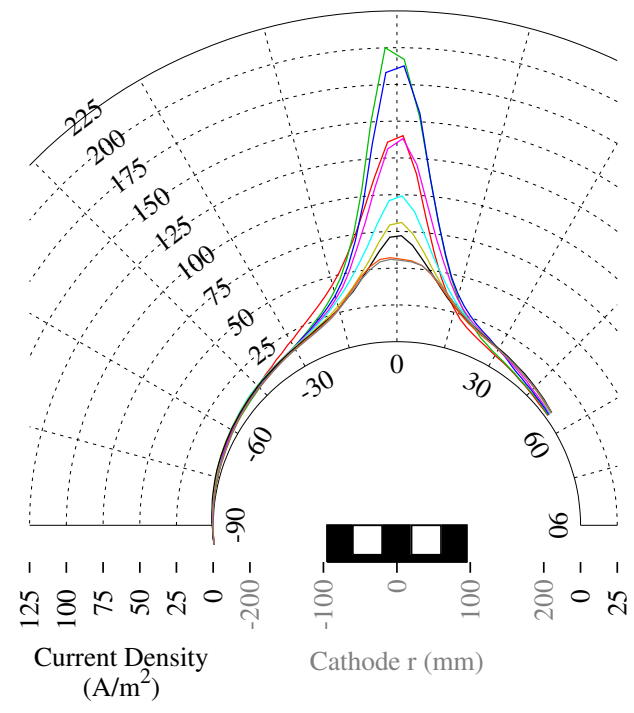

(c) $\dot{m}_{c}=10 \mathrm{SCCM}$

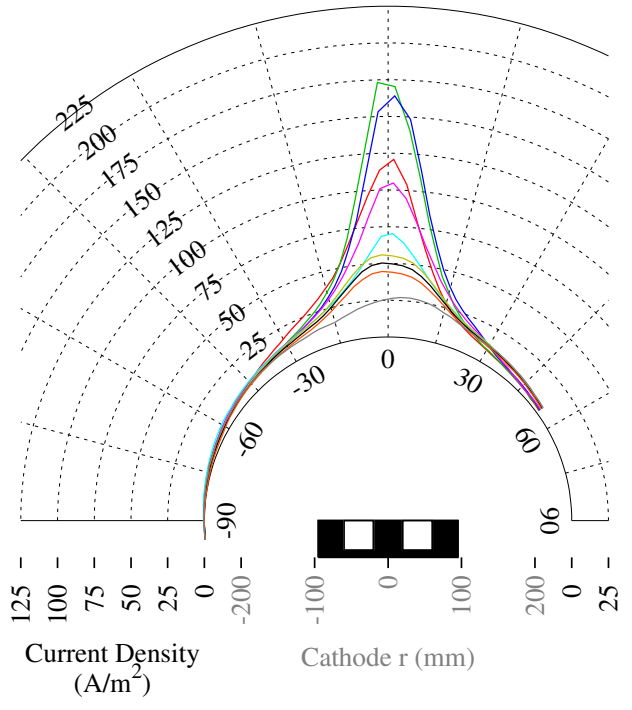

(b) $\dot{m}_{c}=5 \mathrm{SCCM}$

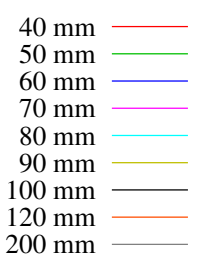

Figure 8.4: Ion beam current density as a function of off-axis angle while using the OOP. Each colored trace represents a different cathode location. 


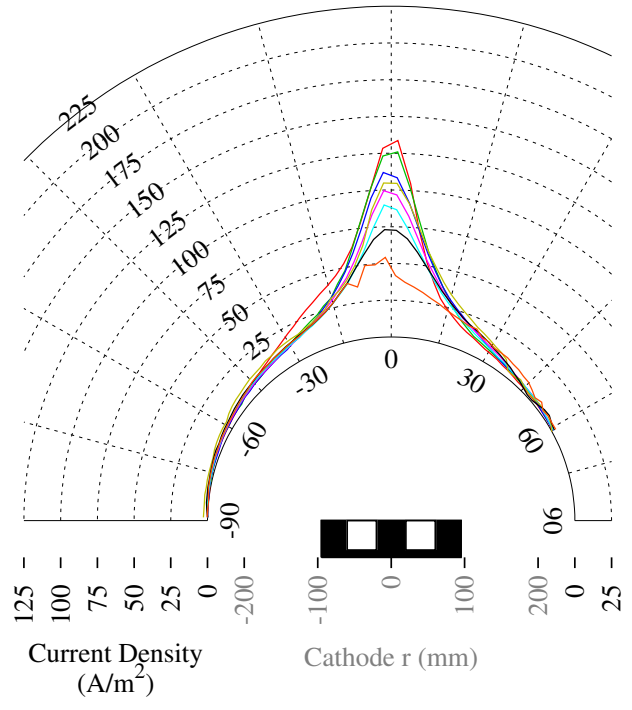

(a) $\dot{m}_{c}=2 \mathrm{SCCM}$

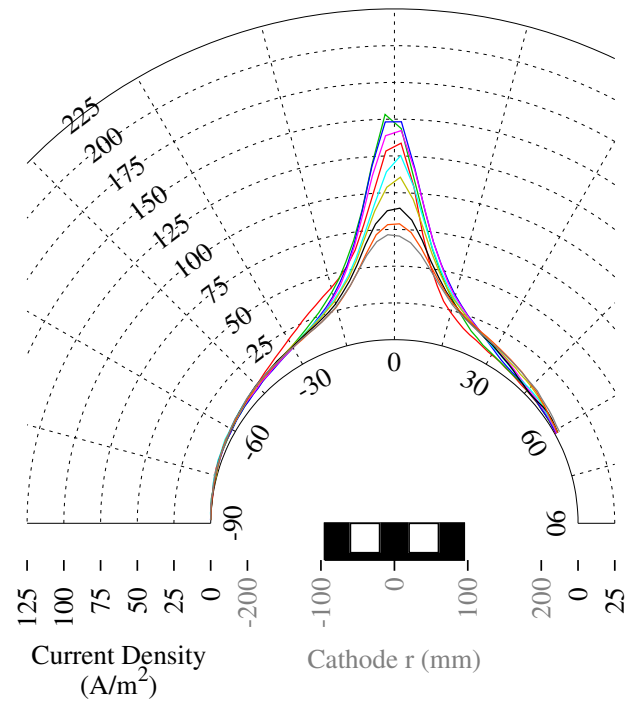

(c) $\dot{m}_{c}=10 \mathrm{SCCM}$

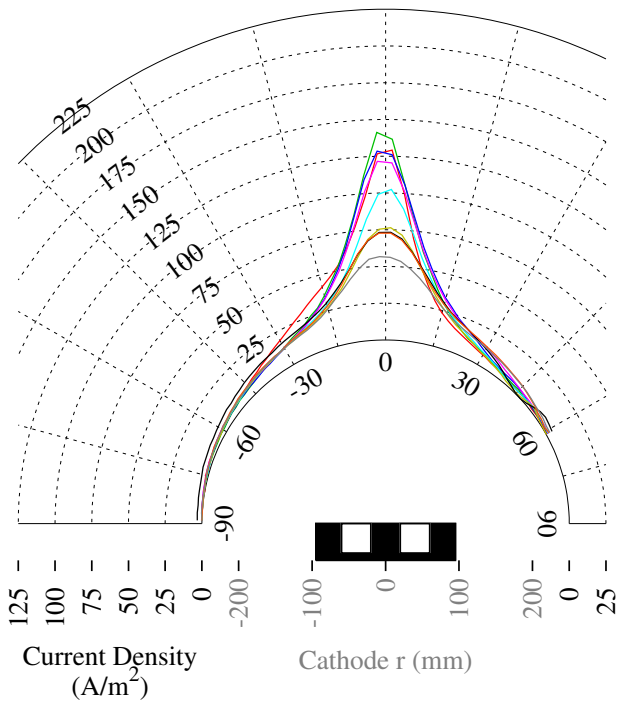

(b) $\dot{m}_{c}=5 \mathrm{SCCM}$

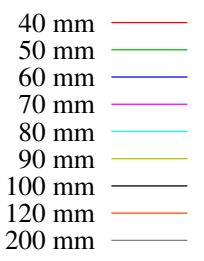

Figure 8.5: Ion beam current density as a function of off-axis angle while using the EOP. Each colored trace represents a different cathode location. 


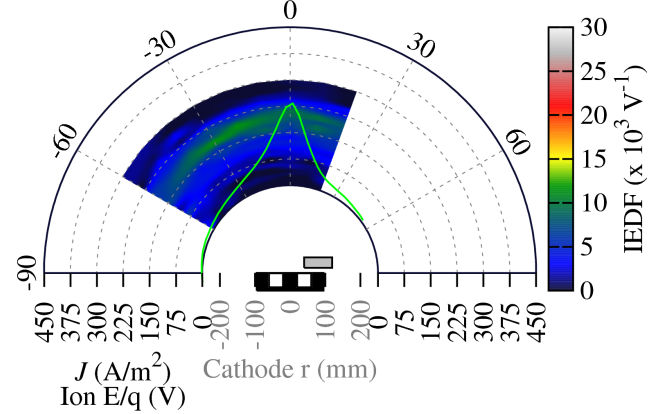

(a) $\dot{m}_{c}=2 \mathrm{SCCM}$, Cathode $\mathrm{r}=40 \mathrm{~mm}$

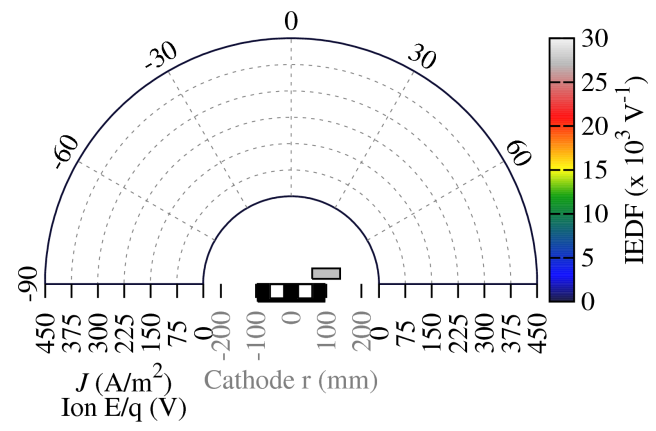

(c) $\dot{m}_{c}=2 \mathrm{SCCM}$, Cathode $\mathrm{r}=60 \mathrm{~mm}$

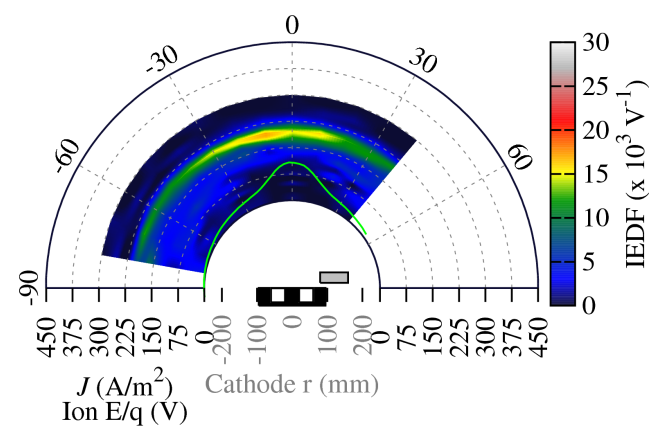

(e) $\dot{m}_{c}=2 \mathrm{SCCM}$, Cathode $\mathrm{r}=80 \mathrm{~mm}$

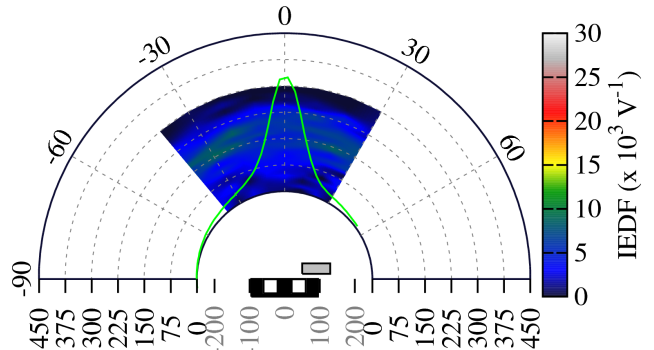

$\underset{\text { Ion } \mathrm{E} / \mathrm{q}(\mathrm{V})}{J(\mathrm{~m})} \quad$ Cathode $\mathrm{r}(\mathrm{mm})$

(b) $\dot{m}_{c}=2 \mathrm{SCCM}$, Cathode $\mathrm{r}=50 \mathrm{~mm}$

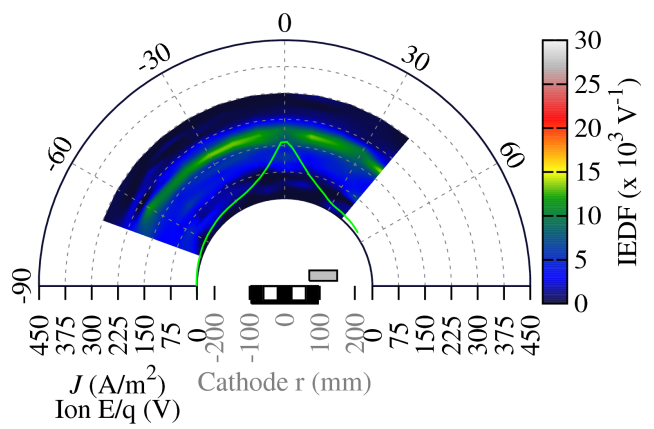

(d) $\dot{m}_{c}=2$ SCCM, Cathode r $=70 \mathrm{~mm}$

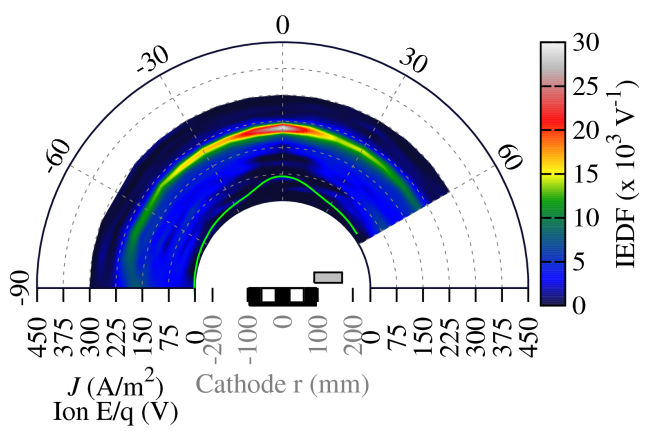

(f) $\dot{m}_{c}=2 \mathrm{SCCM}$, Cathode $\mathrm{r}=90 \mathrm{~mm}$

Figure 8.6: Ion energy distributions and ion current density as a function of angle for cathode mass flow rates of $2 \mathrm{SCCM}$ with the $\mathrm{OOP}$ 


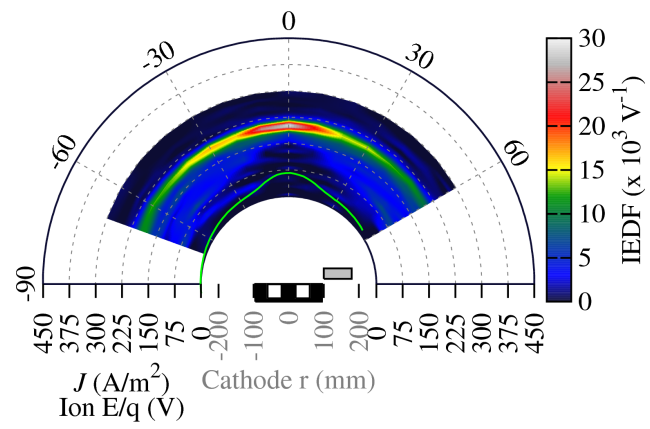

(g) $\dot{m}_{c}=2$ SCCM, Cathode r $=100 \mathrm{~mm}$

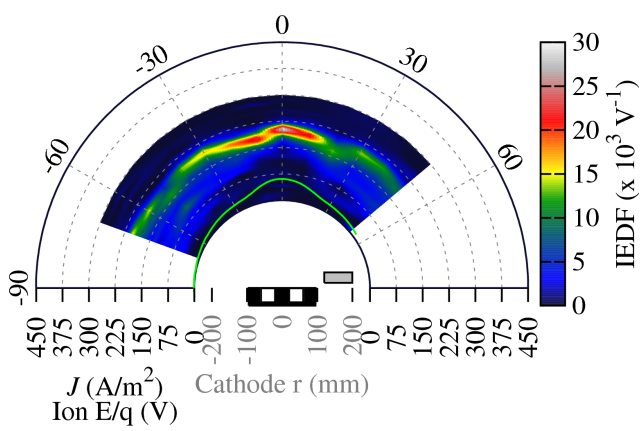

(h) $\dot{m}_{c}=2$ SCCM, Cathode r $=120 \mathrm{~mm}$

Figure 8.6: Ion energy distributions and ion current density as a function of angle for cathode mass flow rates of $2 \mathrm{SCCM}$ with the OOP (continued) 


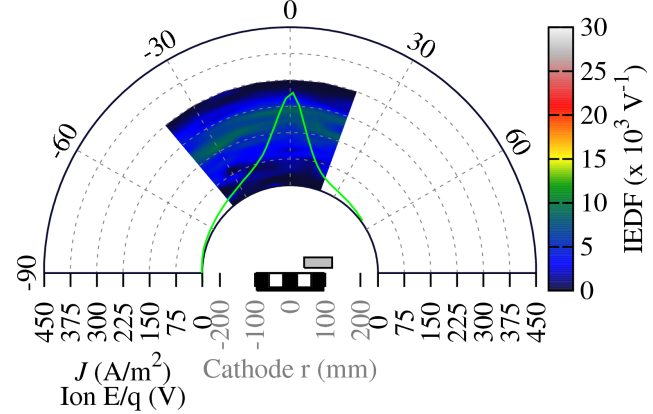

(a) $\dot{m}_{c}=5 \mathrm{SCCM}$, Cathode $\mathrm{r}=40 \mathrm{~mm}$

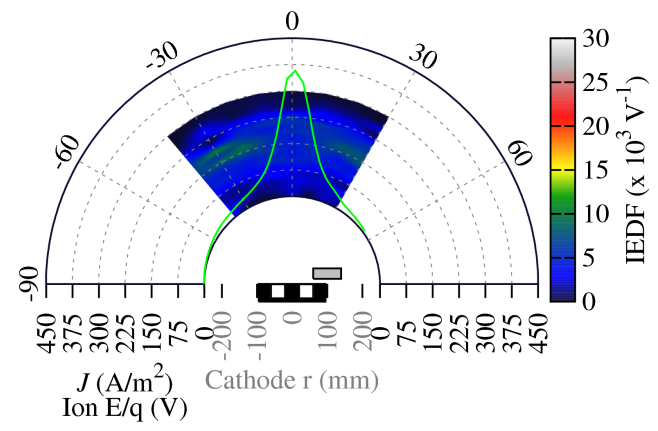

(c) $\dot{m}_{c}=5 \mathrm{SCCM}$, Cathode $\mathrm{r}=60 \mathrm{~mm}$

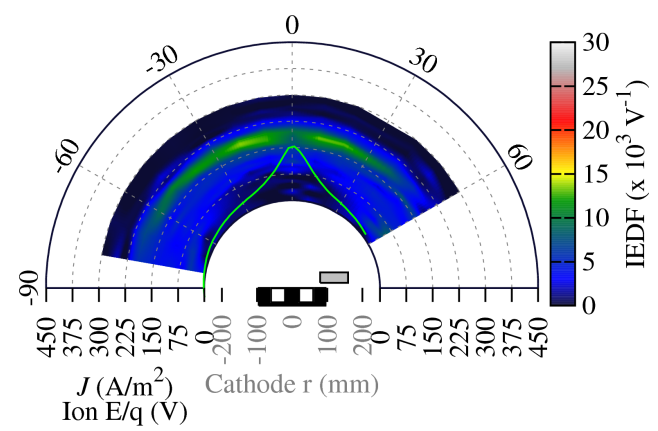

(e) $\dot{m}_{c}=5 \mathrm{SCCM}$, Cathode $\mathrm{r}=80 \mathrm{~mm}$

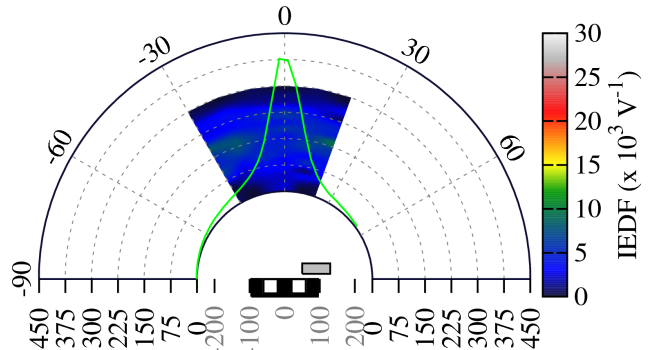

$\underset{\text { Ion E/q (V) }}{J\left(\mathrm{~A} / \mathrm{m}^{2}\right)}$ Cathode $\mathrm{r}(\mathrm{mm})$

(b) $\dot{m}_{c}=5 \mathrm{SCCM}$, Cathode $\mathrm{r}=50 \mathrm{~mm}$

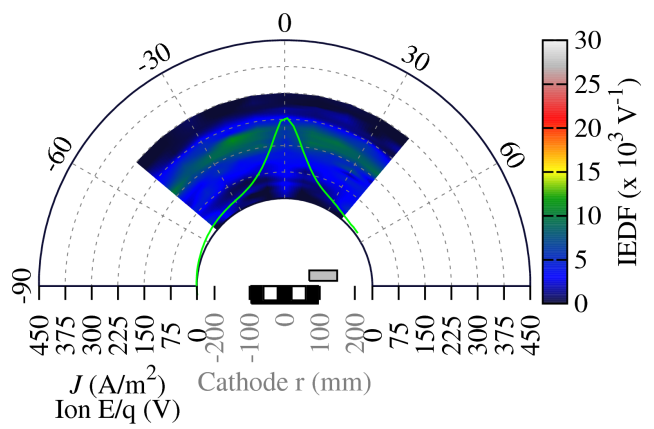

(d) $\dot{m}_{c}=5$ SCCM, Cathode r $=70 \mathrm{~mm}$

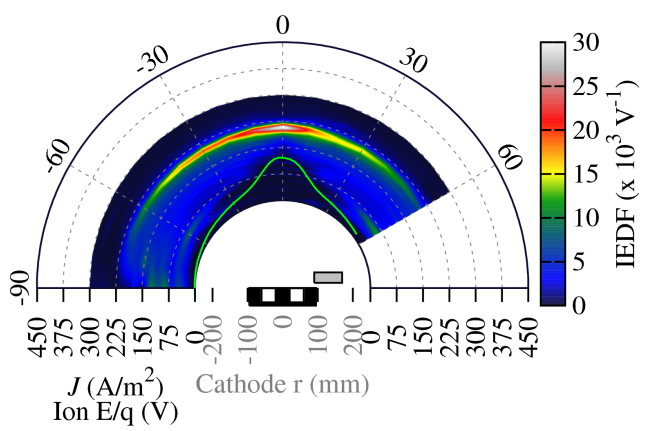

(f) $\dot{m}_{c}=5 \mathrm{SCCM}$, Cathode $\mathrm{r}=90 \mathrm{~mm}$

Figure 8.7: Ion energy distributions and ion current density as a function of angle for cathode mass flow rates of $5 \mathrm{SCCM}$ with the OOP 


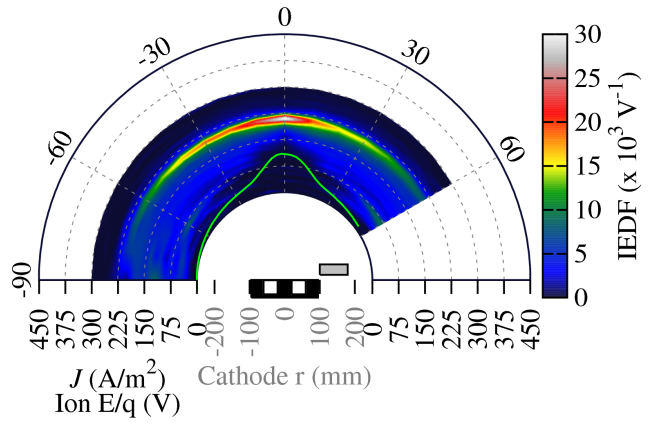

(g) $\dot{m}_{c}=5$ SCCM, Cathode r $=100 \mathrm{~mm}$

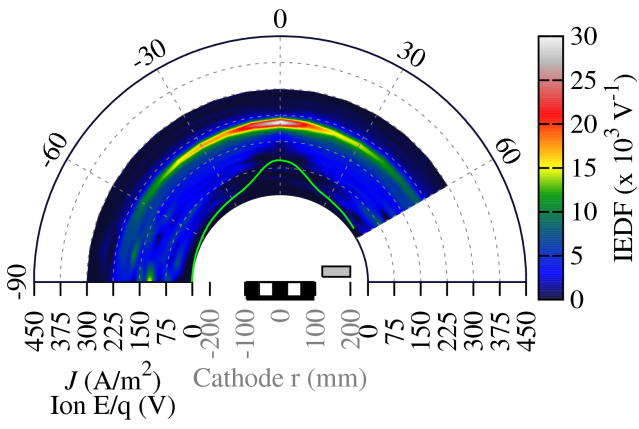

(h) $\dot{m}_{c}=5$ SCCM, Cathode $\mathrm{r}=120 \mathrm{~mm}$

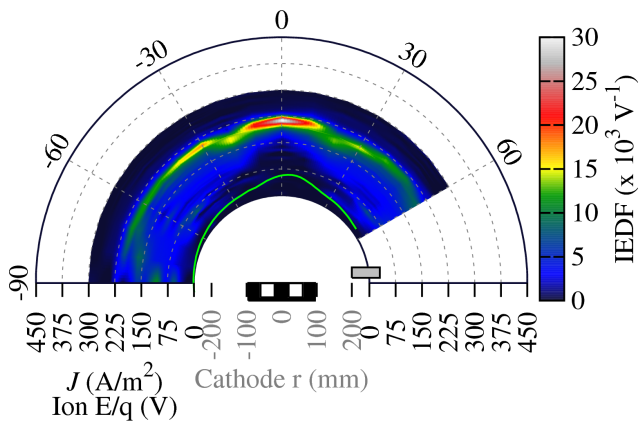

(i) $\dot{m}_{c}=5$ SCCM, Cathode $\mathrm{r}=200 \mathrm{~mm}$

Figure 8.7: Ion energy distributions and ion current density as a function of angle for cathode mass flow rates of $5 \mathrm{SCCM}$ with the OOP (continued) 


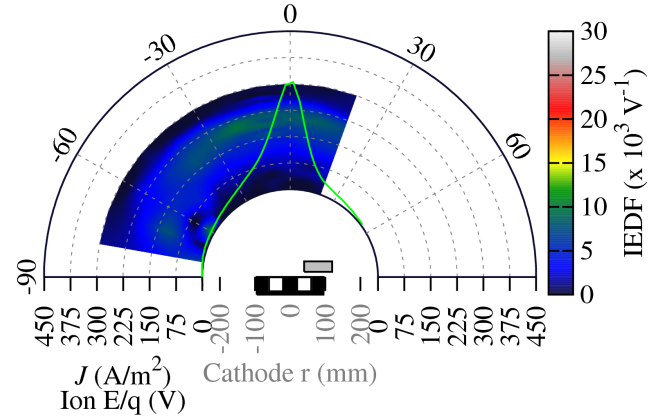

(a) $\dot{m}_{c}=10 \mathrm{SCCM}$, Cathode $\mathrm{r}=40 \mathrm{~mm}$

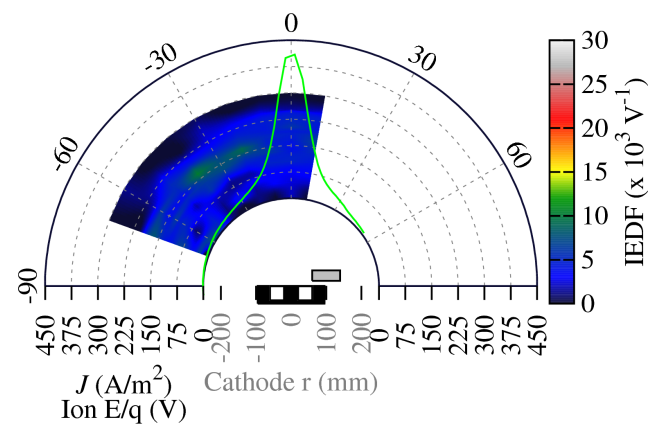

(c) $\dot{m}_{c}=10$ SCCM, Cathode $\mathrm{r}=60 \mathrm{~mm}$

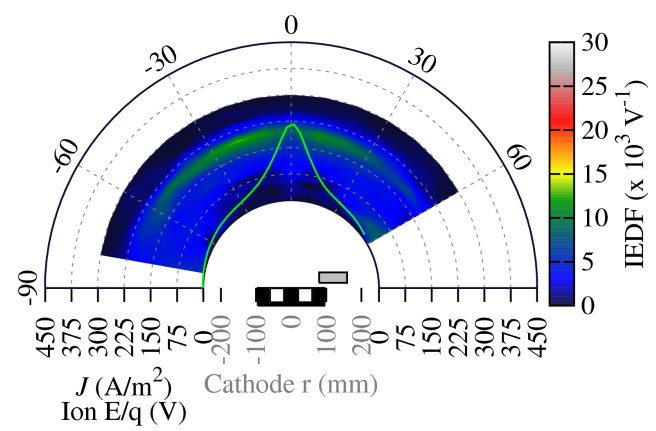

(e) $\dot{m}_{c}=10$ SCCM, Cathode r $=80 \mathrm{~mm}$

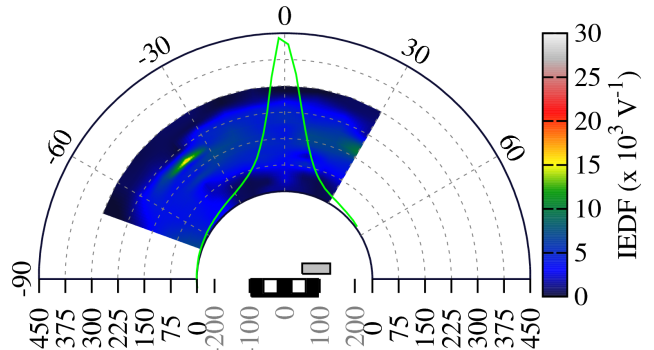

$\underset{\mathrm{Ion} \mathrm{E} / \mathrm{q}(\mathrm{V})}{J(\mathrm{~V})}$ Cathode $\mathrm{r}(\mathrm{mm})$

(b) $\dot{m}_{c}=10 \mathrm{SCCM}$, Cathode $\mathrm{r}=50 \mathrm{~mm}$

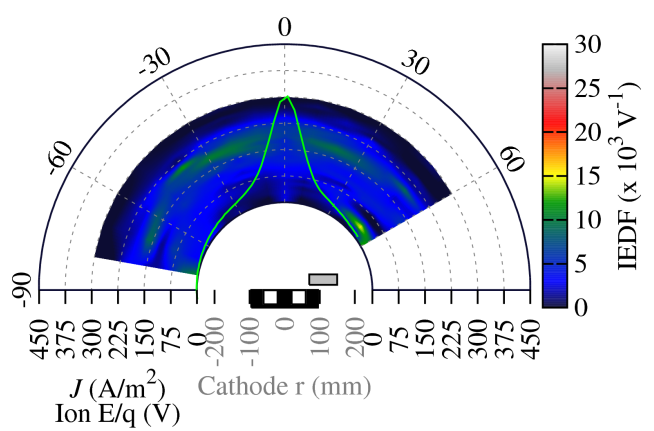

(d) $\dot{m}_{c}=10$ SCCM, Cathode r $=70 \mathrm{~mm}$

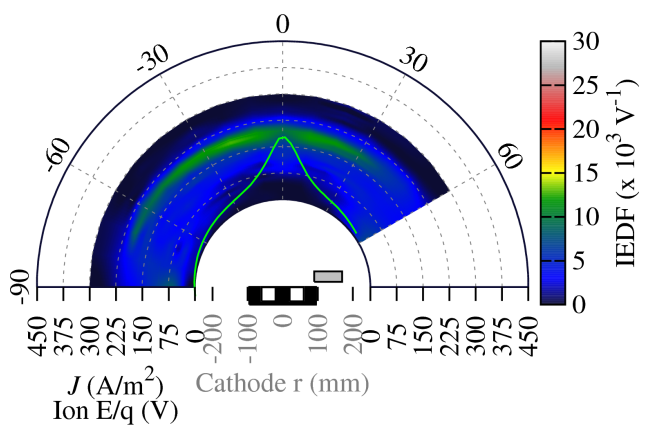

(f) $\dot{m}_{c}=10$ SCCM, Cathode r $=90 \mathrm{~mm}$

Figure 8.8: Ion energy distributions and ion current density as a function of angle for cathode mass flow rates of $10 \mathrm{SCCM}$ with the OOP 


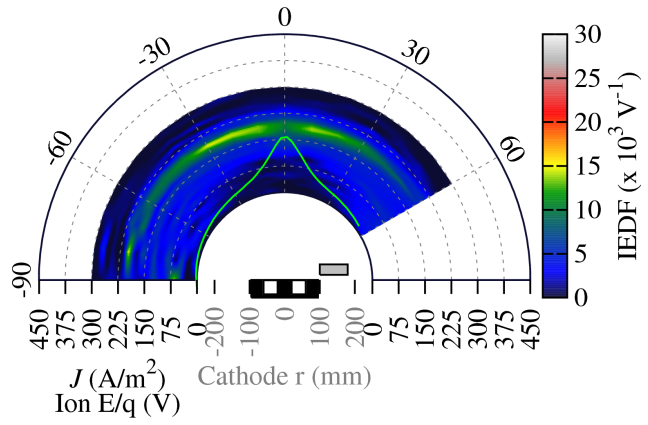

(g) $\dot{m}_{c}=10$ SCCM, Cathode $\mathrm{r}=100 \mathrm{~mm}$

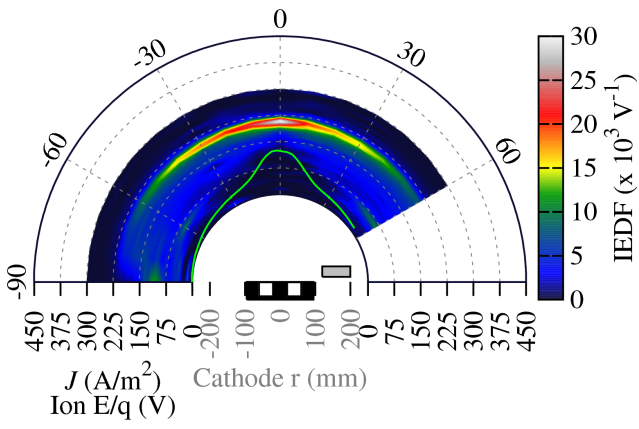

(h) $\dot{m}_{c}=10$ SCCM, Cathode r $=120 \mathrm{~mm}$

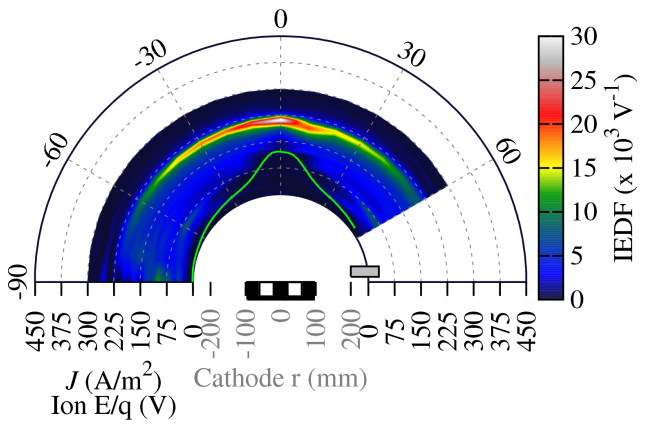

(i) $\dot{m}_{c}=10 \mathrm{SCCM}$, Cathode $\mathrm{r}=200 \mathrm{~mm}$

Figure 8.8: Ion energy distributions and ion current density as a function of angle for cathode mass flow rates of $10 \mathrm{SCCM}$ with the OOP (continued) 


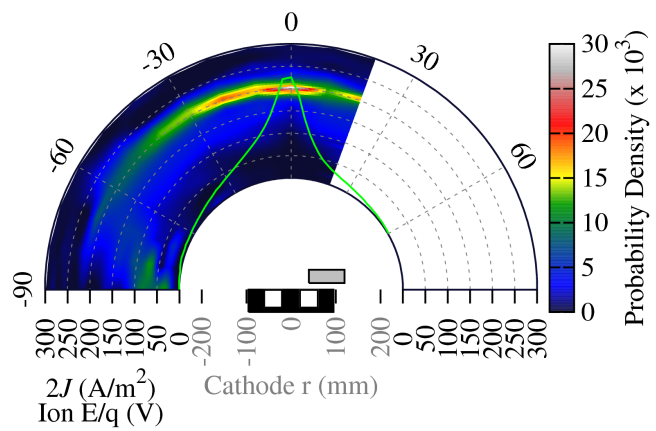

(a) $\dot{m}_{c}=2 \mathrm{SCCM}$, Cathode $\mathrm{r}=40 \mathrm{~mm}$

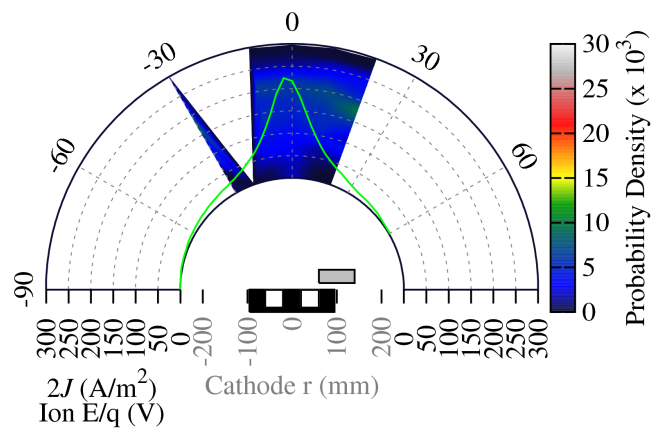

(c) $\dot{m}_{c}=2 \mathrm{SCCM}$, Cathode $\mathrm{r}=60 \mathrm{~mm}$

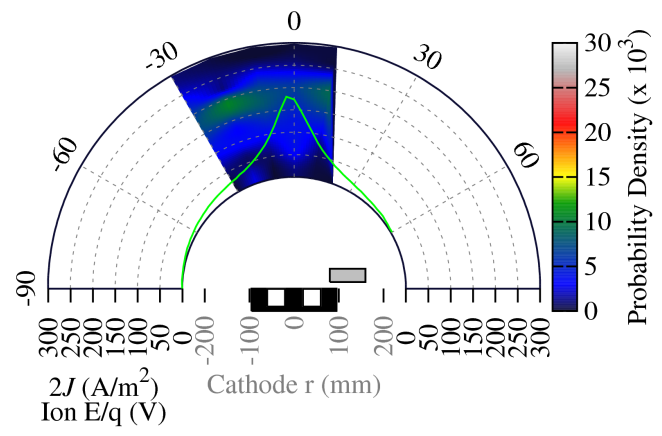

(e) $\dot{m}_{c}=2 \mathrm{SCCM}$, Cathode $\mathrm{r}=80 \mathrm{~mm}$

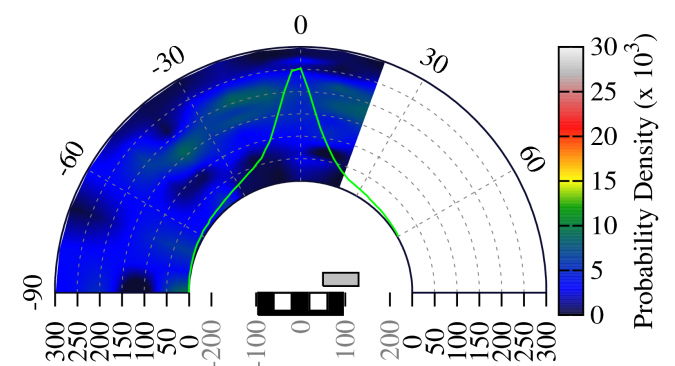

$2 J\left(\mathrm{~A} / \mathrm{m}^{2}\right)$
$\operatorname{Ion} \mathrm{E} / \mathrm{q}(\mathrm{V})$

(b) $\dot{m}_{c}=2 \mathrm{SCCM}$, Cathode $\mathrm{r}=50 \mathrm{~mm}$

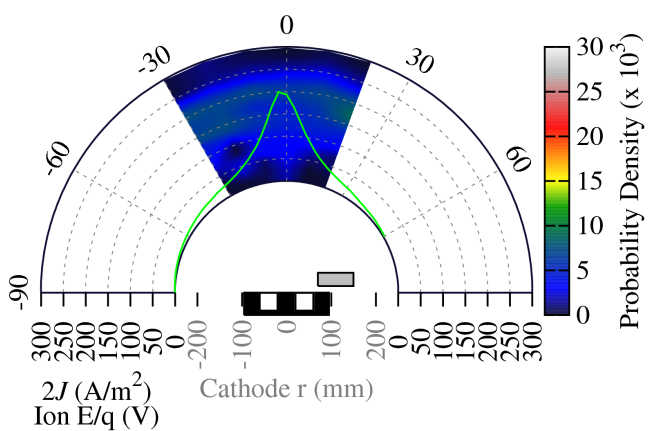

(d) $\dot{m}_{c}=2 \mathrm{SCCM}$, Cathode $\mathrm{r}=70 \mathrm{~mm}$

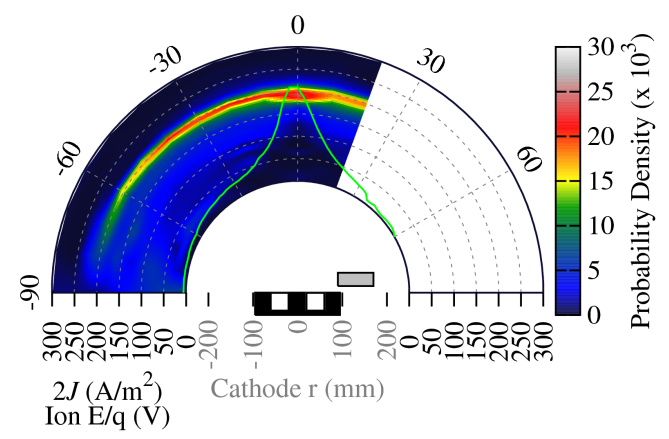

(f) $\dot{m}_{c}=2 \mathrm{SCCM}$, Cathode r $=90 \mathrm{~mm}$

Figure 8.9: Ion energy distributions and ion current density as a function of angle for cathode mass flow rates of $2 \mathrm{SCCM}$ with the EOP 


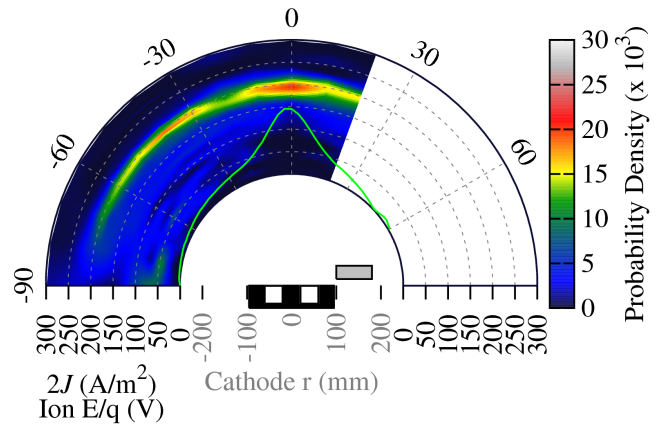

(g) $\dot{m}_{c}=2$ SCCM, Cathode r $=100 \mathrm{~mm}$

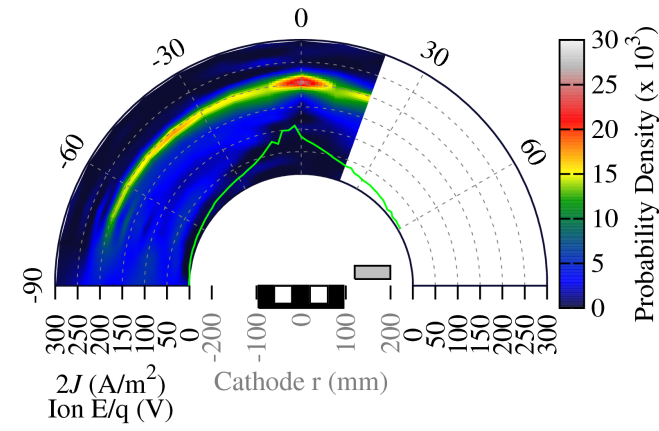

(h) $\dot{m}_{c}=2$ SCCM, Cathode $\mathrm{r}=120 \mathrm{~mm}$

Figure 8.9: Ion energy distributions and ion current density as a function of angle for cathode mass flow rates of $2 \mathrm{SCCM}$ with the EOP (continued) 


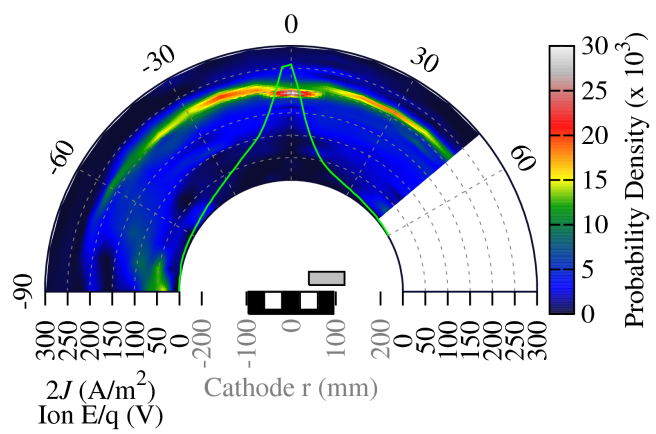

(a) $\dot{m}_{c}=5 \mathrm{SCCM}$, Cathode $\mathrm{r}=40 \mathrm{~mm}$

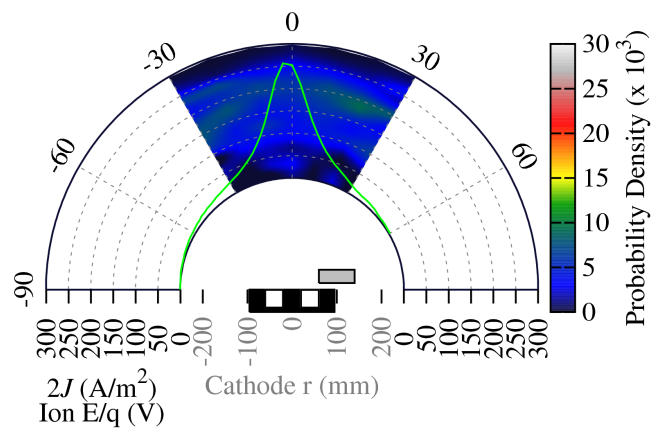

(c) $\dot{m}_{c}=5 \mathrm{SCCM}$, Cathode $\mathrm{r}=60 \mathrm{~mm}$

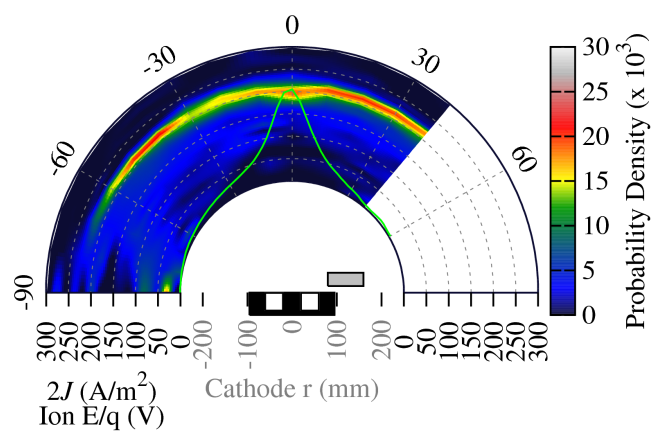

(e) $\dot{m}_{c}=5 \mathrm{SCCM}$, Cathode $\mathrm{r}=80 \mathrm{~mm}$

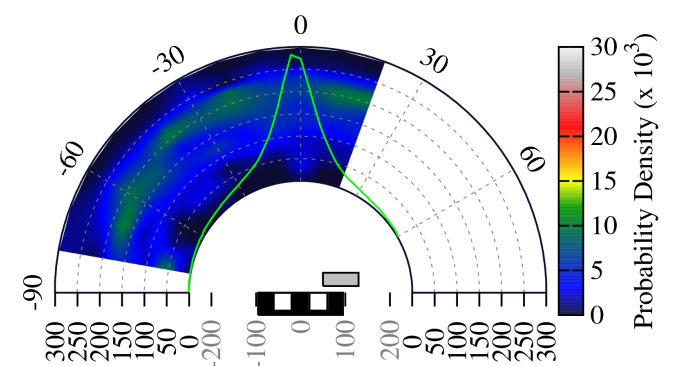

$2 J\left(\mathrm{~A} / \mathrm{m}^{2}\right)$
$\mathrm{Ion} \mathrm{E} / \mathrm{q}(\mathrm{V})$

(b) $\dot{m}_{c}=5 \mathrm{SCCM}$, Cathode $\mathrm{r}=50 \mathrm{~mm}$

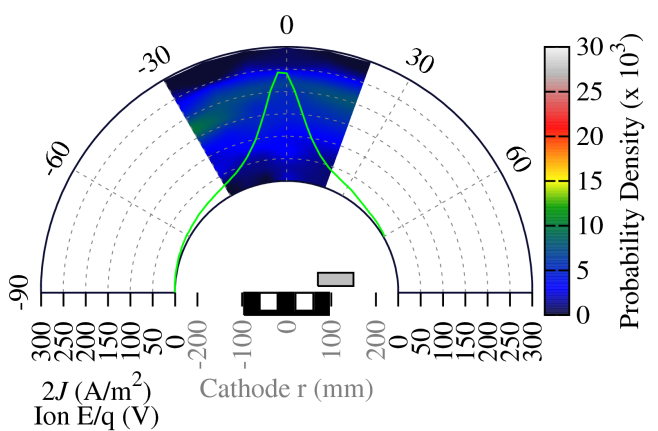

(d) $\dot{m}_{c}=5$ SCCM, Cathode r $=70 \mathrm{~mm}$

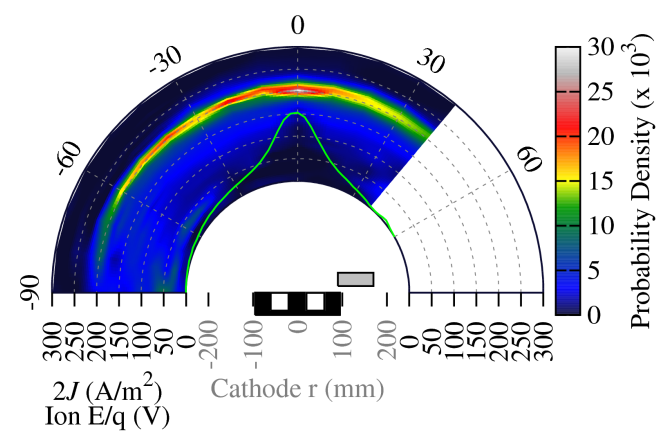

(f) $\dot{m}_{c}=5 \mathrm{SCCM}$, Cathode $\mathrm{r}=90 \mathrm{~mm}$

Figure 8.10: Ion energy distributions and ion current density as a function of angle for cathode mass flow rates of $5 \mathrm{SCCM}$ with the EOP 


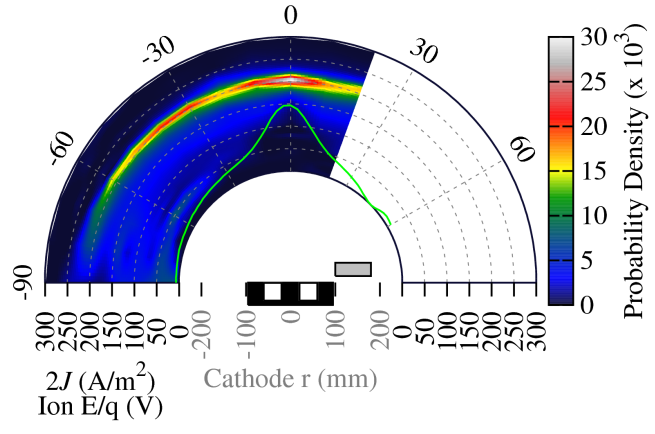

(g) $\dot{m}_{c}=5$ SCCM, Cathode $\mathrm{r}=100 \mathrm{~mm}$

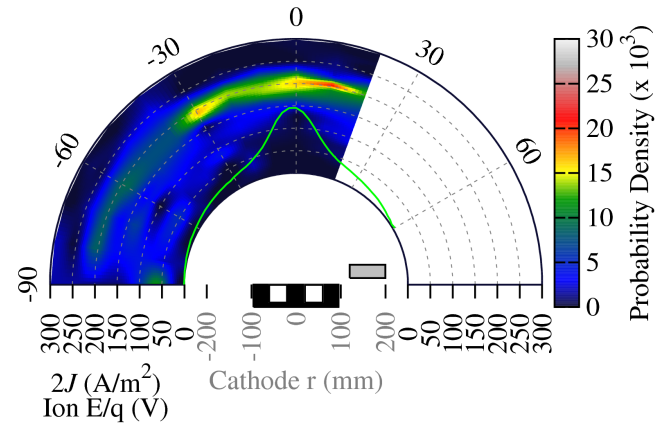

(h) $\dot{m}_{c}=5$ SCCM, Cathode $\mathrm{r}=120 \mathrm{~mm}$

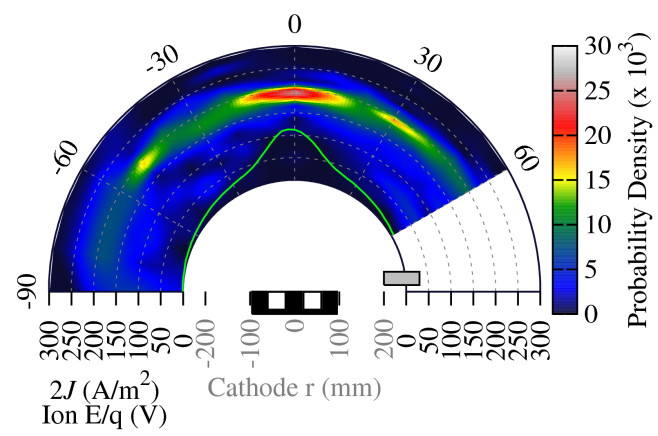

(i) $\dot{m}_{c}=5 \mathrm{SCCM}$, Cathode $\mathrm{r}=200 \mathrm{~mm}$

Figure 8.10: Ion energy distributions and ion current density as a function of angle for cathode mass flow rates of $5 \mathrm{SCCM}$ with the EOP (continued) 


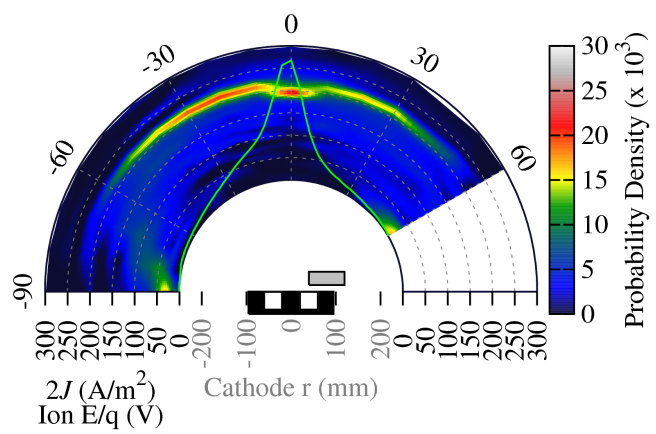

(a) $\dot{m}_{c}=10 \mathrm{SCCM}$, Cathode $\mathrm{r}=40 \mathrm{~mm}$

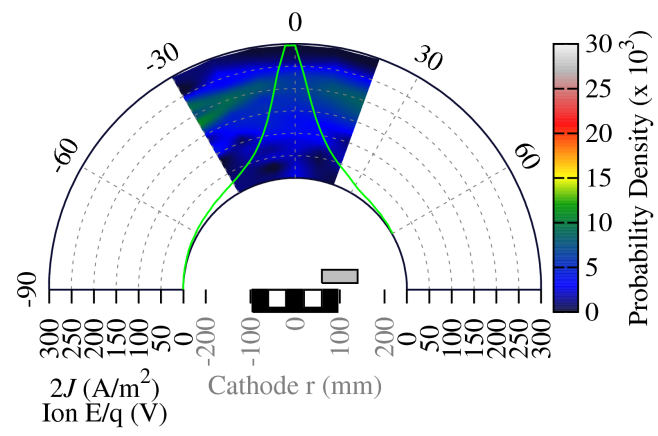

(c) $\dot{m}_{c}=10$ SCCM, Cathode $\mathrm{r}=60 \mathrm{~mm}$

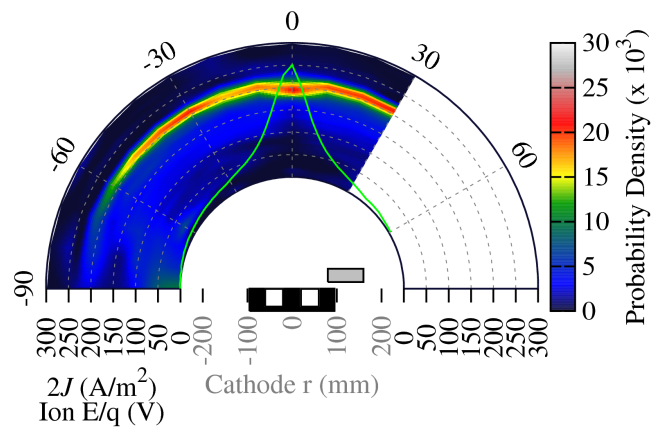

(e) $\dot{m}_{c}=10 \mathrm{SCCM}$, Cathode $\mathrm{r}=80 \mathrm{~mm}$

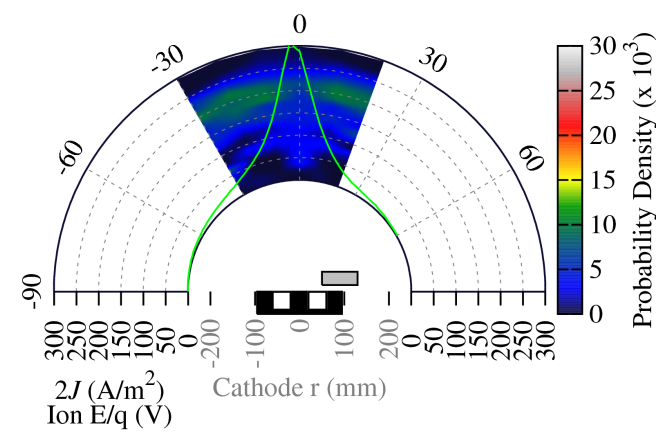

(b) $\dot{m}_{c}=10 \mathrm{SCCM}$, Cathode $\mathrm{r}=50 \mathrm{~mm}$

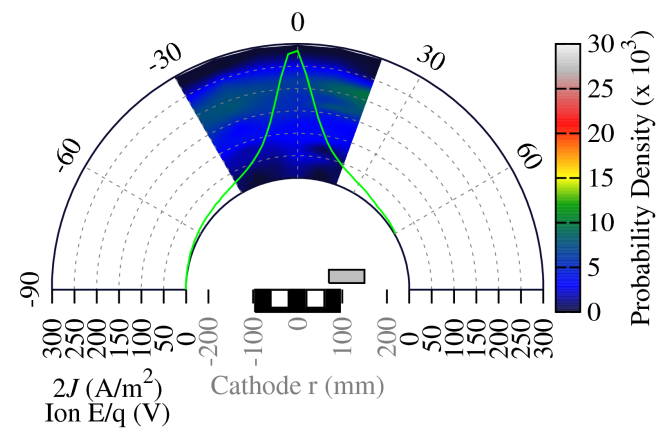

(d) $\dot{m}_{c}=10$ SCCM, Cathode r $=70 \mathrm{~mm}$

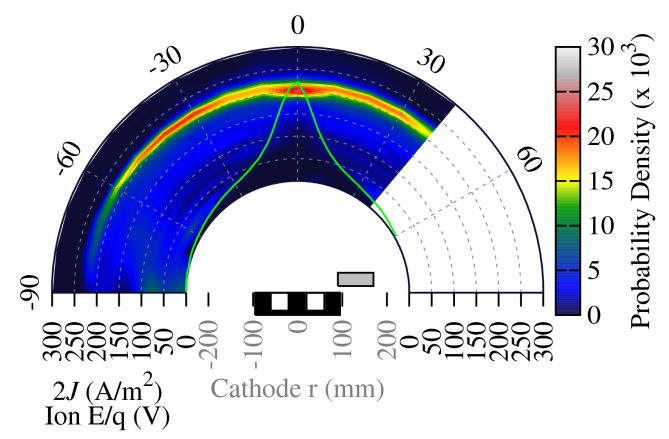

(f) $\dot{m}_{c}=10$ SCCM, Cathode r $=90 \mathrm{~mm}$

Figure 8.11: Ion energy distributions and ion current density as a function of angle for cathode mass flow rates of $10 \mathrm{SCCM}$ with the EOP 


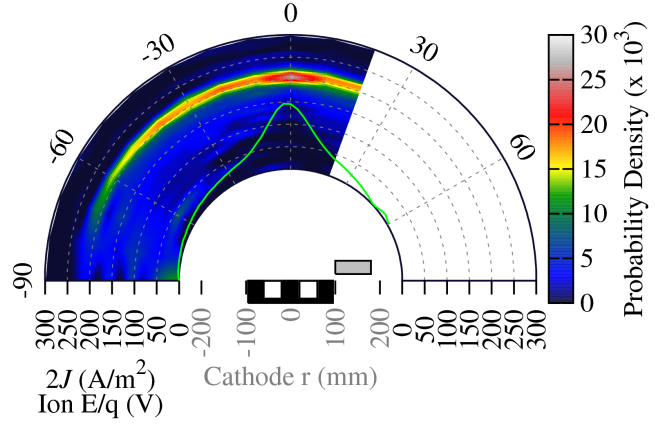

(g) $\dot{m}_{c}=10 \mathrm{SCCM}$, Cathode r $=100 \mathrm{~mm}$

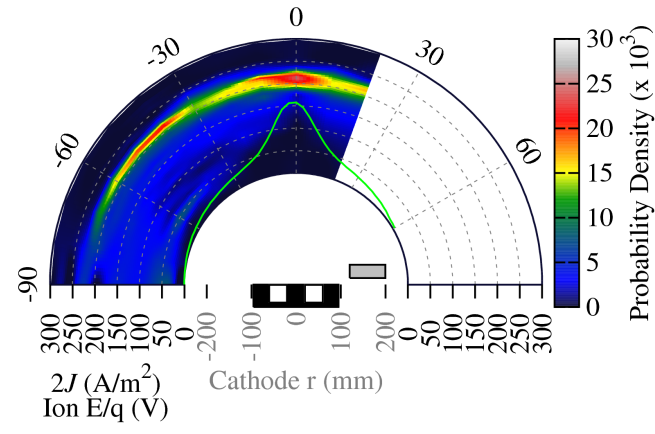

(h) $\dot{m}_{c}=10$ SCCM, Cathode r $=120 \mathrm{~mm}$

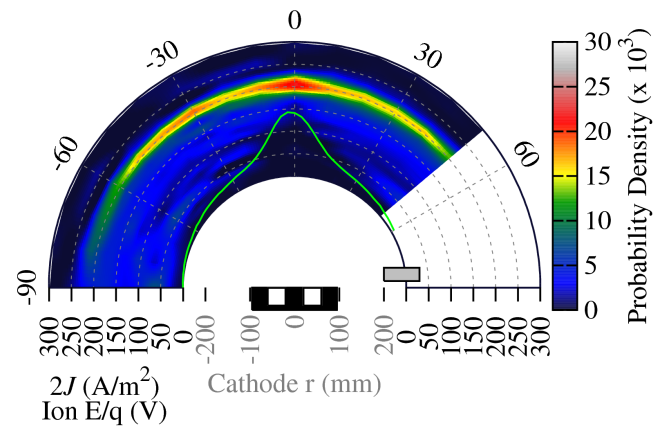

(i) $\dot{m}_{c}=10 \mathrm{SCCM}$, Cathode r $=200 \mathrm{~mm}$

Figure 8.11: Ion energy distributions and ion current density as a function of angle for cathode mass flow rates of 10 SCCM with the EOP (continued) 


\subsection{Efficiency Analysis}

\subsubsection{Method}

With ion current densities and energy distributions it is possible to break down the efficiency into its constituent loss mechanisms with the goal of identifying the significant causes of change in efficiency. Following, Larson ${ }^{3}$ and Ross, ${ }^{4}$ we decompose efficiency into the loss mechanisms shown in Table 8.1. The following series of equations, Equation 8.8 to Equation 8.14, shows how each of these efficiencies are broken out of the total efficiency.

Table 8.1: Efficiency loss mechanisms

\begin{tabular}{|c|c|c|c|c|}
\hline Efficiency & Sym. & Definition & Description & How to Measure \\
\hline $\begin{array}{c}\text { Beam } \\
\text { Divergence }\end{array}$ & $\eta_{\theta}$ & $\langle\cos (\theta)\rangle^{2}$ & $\begin{array}{l}\text { Ion velocity components not } \\
\text { parallel to the thrust axis tend } \\
\text { to cancel and do not generate } \\
\text { thrust }\end{array}$ & $\begin{array}{l}\text { Integrate the Faraday-probe- } \\
\text { derived current densities to } \\
\text { find the expectation value of } \\
\cos (\theta)\end{array}$ \\
\hline $\begin{array}{c}\text { Voltage } \\
\text { Utilization }\end{array}$ & $\eta_{V}$ & $\frac{\frac{1}{2} m\left\langle\mathbf{v}^{2}\right\rangle}{e\left(V_{d}+V_{\mathrm{cg}}\right)}$ & $\begin{array}{l}\text { Not all ionization takes place } \\
\text { at the top of the potential hill. } \\
\text { Because of this, ions do not } \\
\text { receive the full amount of en- } \\
\text { ergy available. }\end{array}$ & $\begin{array}{l}\text { Integrate IEDF and current } \\
\text { density data, similar to the } \\
\text { process for } \eta_{\mathrm{vdf}} \text {, and divide } \\
\text { by the sum of the measured } \\
V_{d} \text { and } V_{\mathrm{cg}}\end{array}$ \\
\hline $\begin{array}{c}\text { Velocity } \\
\text { Distribution }\end{array}$ & $\eta_{\mathrm{vdf}}$ & $\frac{\langle v\rangle^{2}}{\left\langle v^{2}\right\rangle}$ & $\begin{array}{l}\text { Inefficiency due to the spread } \\
\text { in } 1-D \text { velocity space of the } \\
\text { ions }\end{array}$ & $\begin{array}{l}\text { Calculate this efficiency at } \\
\text { each angle from the IEDF. } \\
\text { Perform a weighted average } \\
\text { of all angles, weighting by } \\
\text { current density and solid- } \\
\text { angle. }\end{array}$ \\
\hline $\begin{array}{l}\text { Current } \\
\text { Utilization }\end{array}$ & $\eta_{I}$ & $I_{b} / I_{d}$ & $\begin{array}{l}\text { The "recycle" current "leaks" } \\
\text { from the cathode to the an- } \\
\text { ode without directly creating } \\
\text { thrust in the form of beam } \\
\text { ions. }\end{array}$ & $\begin{array}{l}\text { Integrate Faraday-probe- } \\
\text { derived current densities to } \\
\text { obtain } I_{b} \text { and divide by dis- } \\
\text { charge current }\left(I_{d}\right) \text { measured } \\
\text { by power supply }\end{array}$ \\
\hline $\begin{array}{l}\text { Cathode } \\
\text { Coupling }\end{array}$ & $\eta_{\mathrm{Vcg}}$ & $\frac{V_{d}+V_{\mathrm{cg}}}{V_{d}}$ & $\begin{array}{l}\text { The voltage at the cathode } \\
\text { floats below ground is not } \\
\text { available to accelerate ions. } \\
\text { Note that } V_{\text {cg }} \text { is always neg- } \\
\text { ative. }\end{array}$ & $\begin{array}{l}\text { Measure the cathode cou- } \\
\text { pling voltage }\end{array}$ \\
\hline
\end{tabular}




$$
\begin{aligned}
\eta & =\frac{T^{2}}{2 \dot{m} P} \\
& =\frac{\frac{1}{2} \dot{m}\langle\mathbf{v}\rangle^{2}}{I_{d} V_{d}} \\
& =\frac{\langle\mathbf{v}\rangle^{2}}{\left\langle\mathbf{v}^{2}\right\rangle} \frac{1}{\frac{1}{2}\left\langle\mathbf{v}^{2}\right\rangle} \\
& =\underbrace{\left\langle v^{2}\right\rangle}_{\eta_{\mathrm{vdf}} V_{d}} \underbrace{\langle\cos (\theta)\rangle^{2}}_{\eta_{\theta}} \frac{\frac{1}{2} \dot{m}\left\langle\mathbf{v}^{2}\right\rangle}{I_{d} V_{d}} \\
& =\eta_{\mathrm{vdf}} \eta_{\theta} \underbrace{\frac{e \dot{m} / m}{I_{d}}}_{\eta_{I}} \frac{\frac{1}{2} m\left\langle\mathbf{v}^{2}\right\rangle}{e V_{d}} \\
& =\eta_{\mathrm{vdf}} \eta_{\theta} \underbrace{\frac{I_{b}}{I_{d}}}_{\eta_{I}} \underbrace{\frac{1}{2} m\left\langle\mathbf{v}^{2}\right\rangle}_{\eta_{V}} \frac{\underbrace{}_{\left.V_{d}+V_{\mathrm{cg}}\right)}}{\underbrace{V_{d}}_{V_{d}+V_{\mathrm{cg}}}} \\
& =\eta_{\mathrm{vdf}} \eta_{\theta} \eta_{\eta_{\mathrm{vcg}}}^{\eta_{V}}
\end{aligned}
$$

No attempt is made to correct for ionization fraction in these equations, or the proceding analysis. That is to say, we assume that all ions are singly charged. Because of this, $\eta_{\mathrm{vdf}}$ is probably slightly overstated, while $\eta_{V}$ is understated.

The various expectation values are calculated by a weighted average according to the following equation:

$$
\langle x\rangle=\frac{\int_{-90^{\circ}}^{60^{\circ}} \int_{0}^{300 \mathrm{~V}} x(V, \theta) f(V, \theta) j(\theta) R^{2} \sin (|\theta|) d V d \theta}{\int_{-90^{\circ}}^{60^{\circ}} \int_{0}^{300 \mathrm{~V}} f(V, \theta) j(\theta) R^{2} \sin (|\theta|) d V d \theta} .
$$

Since the data to be integrated are discrete, the inner integral is first performed at each available angle using Simpson's rule. The results of these integrations are then linearly interpolated, multiplied by the current densities, and integrated across $\theta$. Where RPA data are unavailable, the $\theta$ integration range is truncated.

Strictly speaking, the separation of $\eta_{\mathrm{vdf}}$ and $\eta_{\theta}$ in Equation 8.11 is only valid if the IEDF is independent of angle. This is clearly not the case. However, because the IEDF seems to change most at high angles where the current density is small, we will procede with the assumption anyway. 


\subsubsection{Results}

The results of this efficiency decomposition are shown in Figure 8.12, along with the measured efficiency and the calculated total efficiency. The total efficiency is the product of all efficiency components according to Equation 8.14 and is denoted by $\eta_{\text {prod. }}$. Total efficiencies are plotted with solid lines, while efficiency components are plotted with dashes.

Based on the discussion of Faraday probe errors in Section 5.2.4, the absolute uncertainty on the integrated beam currents used to calculate the current utilization efficiency are estimated at $+0 \% /-40 \%$. In other words, the measurement should be thought of as an upper limit of the actual value. This uncertainty propagates directly through to the uncertainty in $\eta_{I}$. The beam divergence is calculated from the same data, but the uncertainty in magnitude of the current density affects the calculation of beam divergence less strongly. Rovey et al. found $90 \%$ beam divergence angles* that were reduced from 30 degrees using an unfiltered probe to between 25 and 28 degrees when using an probe that filtered out CEX ions. ${ }^{5}$ Based on this, I estimate the uncertainty in beam divergence (defined in this work as $\langle$ thet $a\rangle$ ) at 5 degrees. Typical beam divergences in this work are around 35 degrees. Here again, the uncertainty is primarily in the negative direction. That is, actual divergence is probably less than 35 degrees. Propagating the uncertainty through the equation for $\eta_{\theta}$ yields an uncertainty of $+8 \% /-0 \%$.

The uncertainty estimation procedure described in Section 5.3 was used for the efficiency components based on the IEDF, that is, $\eta_{V}$ and $\eta_{\text {vdf }}$. This procedure was done for all of the IEDFs at one of the noisier operating conditions (OOP, $\dot{m}_{c}=5 \mathrm{SCCM}$, $r=70 \mathrm{~mm}$ ). From this, I estimated the uncertainty on $\eta_{V}$ at $\pm 20 \%$ and the uncertainty on $\eta_{\mathrm{vdf}}$ at $\pm 4 \%$.

Proper handling of asymmetric, systematic uncertainties requires knowledge of the the probability distribution functions for the measurements. Unfortunately, for the Faraday-probe-derived data, no such function is available. Therefore, to propagate the errors through to $\eta_{\text {prod }}$ the following procedure is used. The percentage uncertainties resulting from the random errors associated with the $\eta_{V}$ and $\eta_{\mathrm{vdf}}$ are added in quadrature. The random error on $\eta_{\mathrm{Vcg}}$ is negligible compared to these quantities and is disregarded. Then, the systematic errors associated with $\eta_{I}$ and $\eta_{\theta}$ are added linearly. For the positive and negative uncertainties of a quantity $x$ given by $U_{ \pm}(x)$, this is expressed mathematically as

$$
\frac{U_{ \pm}\left(\eta_{\mathrm{prod}}\right)}{\eta_{\mathrm{prod}}}=\sqrt{\left(\frac{U\left(\eta_{V}\right)}{\eta_{V}}\right)^{2}+\left(\frac{U\left(\eta_{\mathrm{vdf}}\right)}{\eta_{\mathrm{vdf}}}\right)^{2}} \pm \frac{U_{ \pm}\left(\eta_{I}\right)}{\eta_{I}} \pm \frac{U_{ \pm}\left(\eta_{\theta}\right)}{\eta_{\theta}}
$$

${ }^{*}$ The $90 \%$ beam divergence angle is the angle to which, when integrating beam current from 0 degrees, will include $90 \%$ of the total beam current. 


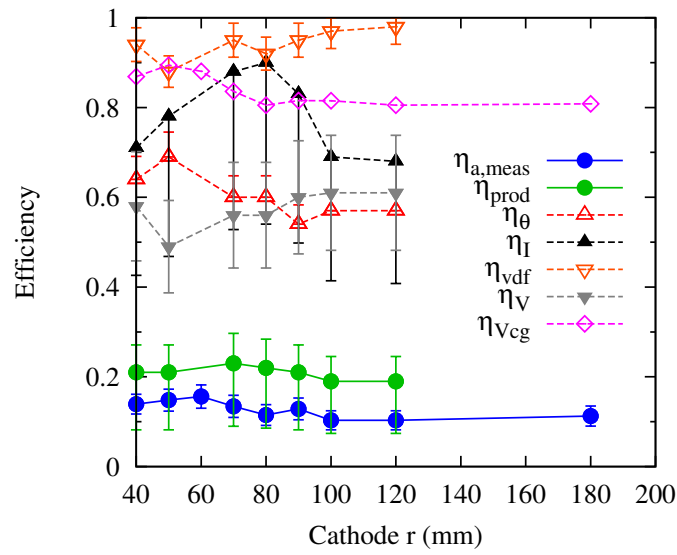

(a) OOP, $\dot{m}_{c}=2 \mathrm{SCCM}$

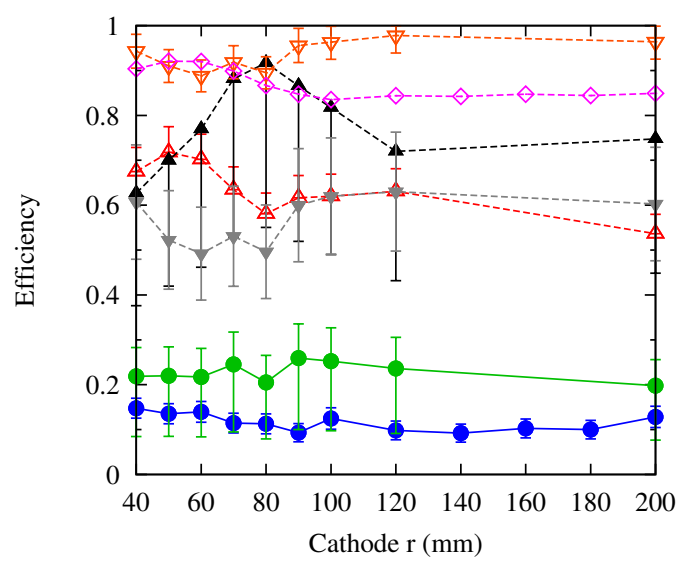

(c) OOP, $\dot{m}_{c}=5 \mathrm{SCCM}$

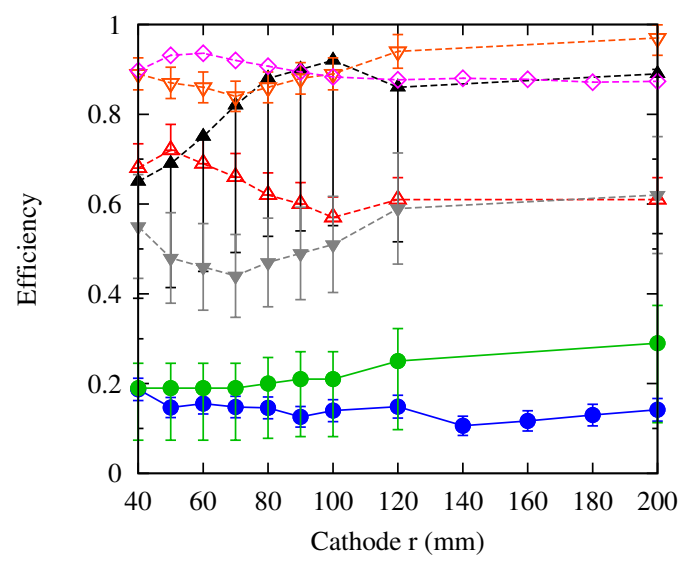

(e) OOP, $\dot{m}_{c}=10 \mathrm{SCCM}$

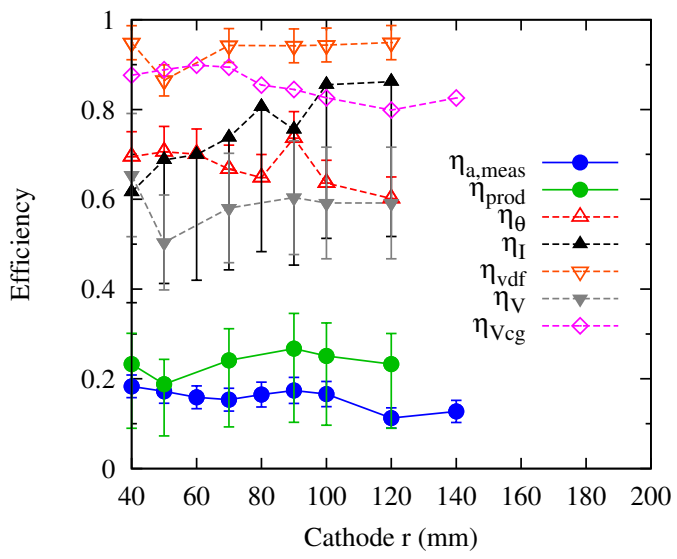

(b) EOP, $\dot{m}_{c}=2 \mathrm{SCCM}$

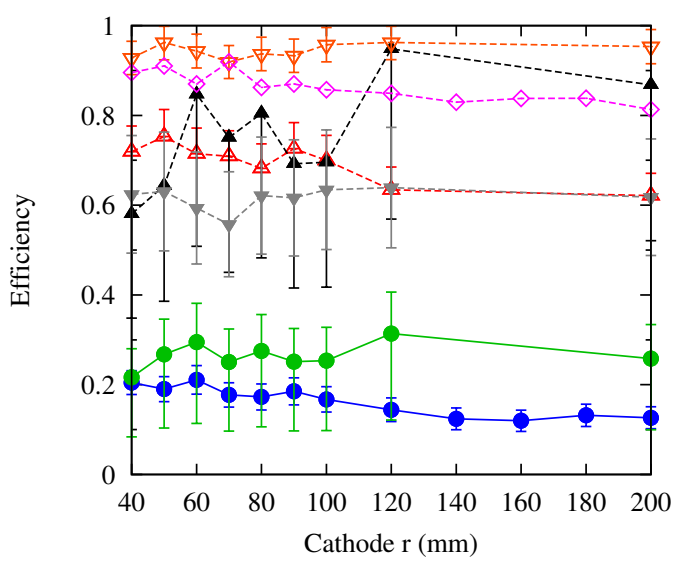

(d) EOP, $\dot{m}_{c}=5 \mathrm{SCCM}$

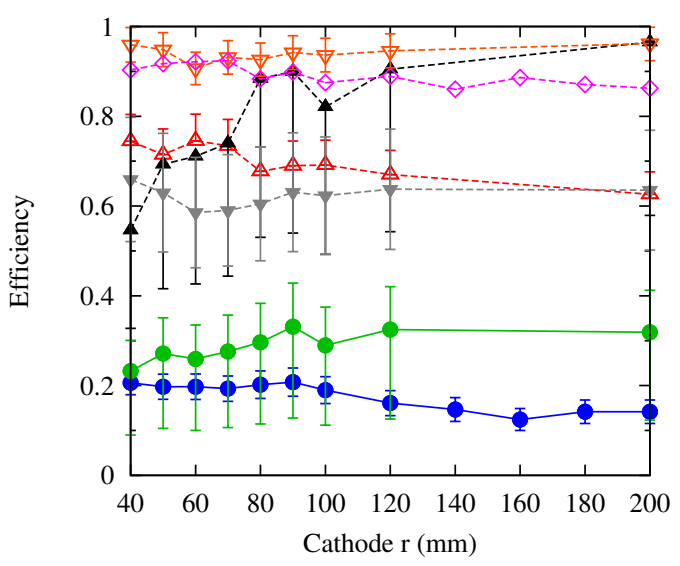

(f) EOP, $\dot{m}_{c}=10 \mathrm{SCCM}$

Figure 8.12: Efficiency component breakdown 
This method is guaranteed to not understate the propagated uncertainty and likely overstates it. The results of the propagation are displayed in Figure 8.12.

\subsubsection{Discussion}

Inefficiency in voltage utilization and beam divergence are the most significant contributions to the inefficiency of the thruster, across all cases. The voltage utilization is seen to decrease slowly as the cathode is brought in from $200 \mathrm{~mm}$, decrease more rapidly starting at $120 \mathrm{~mm}$ to a minimum located near $60 \mathrm{~mm}$ and then increase as the cathode is brought to $40 \mathrm{~mm}$. The trends in velocity distribution efficiency follow closely that of $\eta_{V}$. These trends could be explained by increased cathode propellent ingestion as the cathode is brought closer. Ingested cathode neutrals are likely to be ionized lower in the potential hill, before they drift back into the higher potential regions inside the discharge chamber. The lower energies of the cathode ions mixed in with the anode ions would result in both a lower $\eta_{V}$ and a lower $\eta_{\text {vdf }}$. Additional evidence for this interpretation can be found in that the depth and breadth of the decrease in these efficiency components between $40 \mathrm{~mm}$ and $100 \mathrm{~mm}$ increases with increasing $\dot{m}_{c}$, at least for the OOP data. If this interpretation is correct, it should be noted that the decrease in this efficiency component is a little misleading. The addition of the impulse of the low velocity ions to the thrust of the system is still an increase in thrust, and is better than simply having the cathode neutrals expelled into space.

Furthermore, the decrease in $\eta_{V}$ should be offset by an increase in $\eta_{I}$, as both $I_{b}$ increases by, at most, the same amount as $I_{d}{ }^{*}$ Indeed this is seen in some of the cases, particularly of the OOP data, but not in all. The current utilization efficiency for the OOP exhibits a bump between $40 \mathrm{~mm}$ and $120 \mathrm{~mm}$, with the maximum occurring between $80 \mathrm{~mm}$ and $100 \mathrm{~mm}$. The trends are less clear for the EOP data. The bump may be explained in part by an increase in cathode propellent ingestion. However, it is too large for this to be the only effect, particularly in the 2 SCCM OOP case. As discussed in terms of discharge current in Section 8.3.2 the maximum increase in current (both $I_{b}$ and $I_{d}$ ) possible is $0.14 \mathrm{~A}$. At greater radial cathode positions the discharge currents are approximately $3 \mathrm{~A}$ and $\eta_{I}$ is approximately 0.7 . If all of the cathode propellent was ingested and ionized, this would result in an improvement in efficiency from 0.7 to 0.71 , according to:

$$
\eta_{I, \text { improved }}=\frac{I_{b, \text { improved }}}{I_{d, \text { improved }}}=\frac{\eta_{I} I_{d}+I_{\text {ingested }}}{I_{d}+I_{\text {ingested }}}
$$

*This assumes that every cathode neutral ingested and ionized is captured by the Faraday probe. If that is true, then $\eta_{I}$ must increase since, $\frac{I_{b}+I_{\text {ingested }}}{I_{d}+I_{\text {ingested }}}>\frac{I_{b}}{I_{d}}$, for $I_{b}<I_{d}$. 
Even in the 10 SCCM case only about a 5\% improvement is possible. Clearly another process is at work both on $\eta_{I}, \eta_{V}$, and $\eta_{\mathrm{vdf}}$. A possibility will be discussed in the conclusion.

The beam divergence efficiency has nearly an opposite trend to that of $\eta_{V}$. It is relatively flat beyond $120 \mathrm{~mm}$ and exhibiting a bump between $40 \mathrm{~mm}$ and $120 \mathrm{~mm}$. One also notes that $\eta_{\mathrm{Vcg}}$ varies almost in lock-step with $\eta_{\theta}$. This will be further explored in Section 8.6 and in the conclusion.

All efficiency components except $\eta_{I}$ are consistently higher for the EOP than for the OOP. Furthermore, the trends that are similar between the EOP and the OOP seem shallower and broader for the EOP. This suggests that the position of the separatrix may be playing a roll in the efficiency. It is also possible, however, that this is merely an effect of the differing magnetic fields used in the two experiments.

\subsection{Plume Properties}

\subsubsection{Results}

Figures 8.13 and 8.14 show sample results from the measurement of the plume properties. These figures plot $T_{e}, n_{e}$, and $V_{f}$. Using these measurements and Equation $5.22, V_{p}$ was calculated and the results were plotted. All probe traces were reduced using the Peterson and Talbot analysis, as discussed in Section 5.4. The modelled magnetic field lines and magnitude contours (in millitesla) are overlaid in solid and dashed lines, respectively (see Chapter 6 for model details). A sketch of the thruster and the cathode show the position of the plume data with respect to these objects. The units of the $r$ and $z$ axes are millimeters. Note, also, that the electron density scale is logarithmic. The white points superimposed on the figures indicate the positions at which the probe sampled the plume.* The cost score (Equation 5.29) was used to automatically reject any trace which did not sufficiently match the probe theory. A value of 0.3 was chosen as the maximum allowable cost after manually inspecting several traces and their theoretical fits. Note that this rejection only applies to the values for $T_{e}, n_{e}$, and $V_{p}$. The value of $V_{f}$ is a single-point measurement and, therefore, independent of the quality of the match between the measured probe trace and theory. The plasma property contours were created by interpolating the accepted data points onto a $1 \mathrm{~mm}$ rectangular mesh using a natural neighbor interpolation. ${ }^{6}$ The full results for every operating condition measured are presented in Appendix C.

Electrostatic probe data are notoriously inaccurate. It is likely that the largest contribution to the error in these probe measurements comes from errors due to probe heating. A detailed exploration of the probe heating effects in this experiment can be found in

${ }^{*}$ The choice of white for these points is good for the majority of the area of each figure. However, some points at the edges of the figures may be difficult or impossible to discern. 

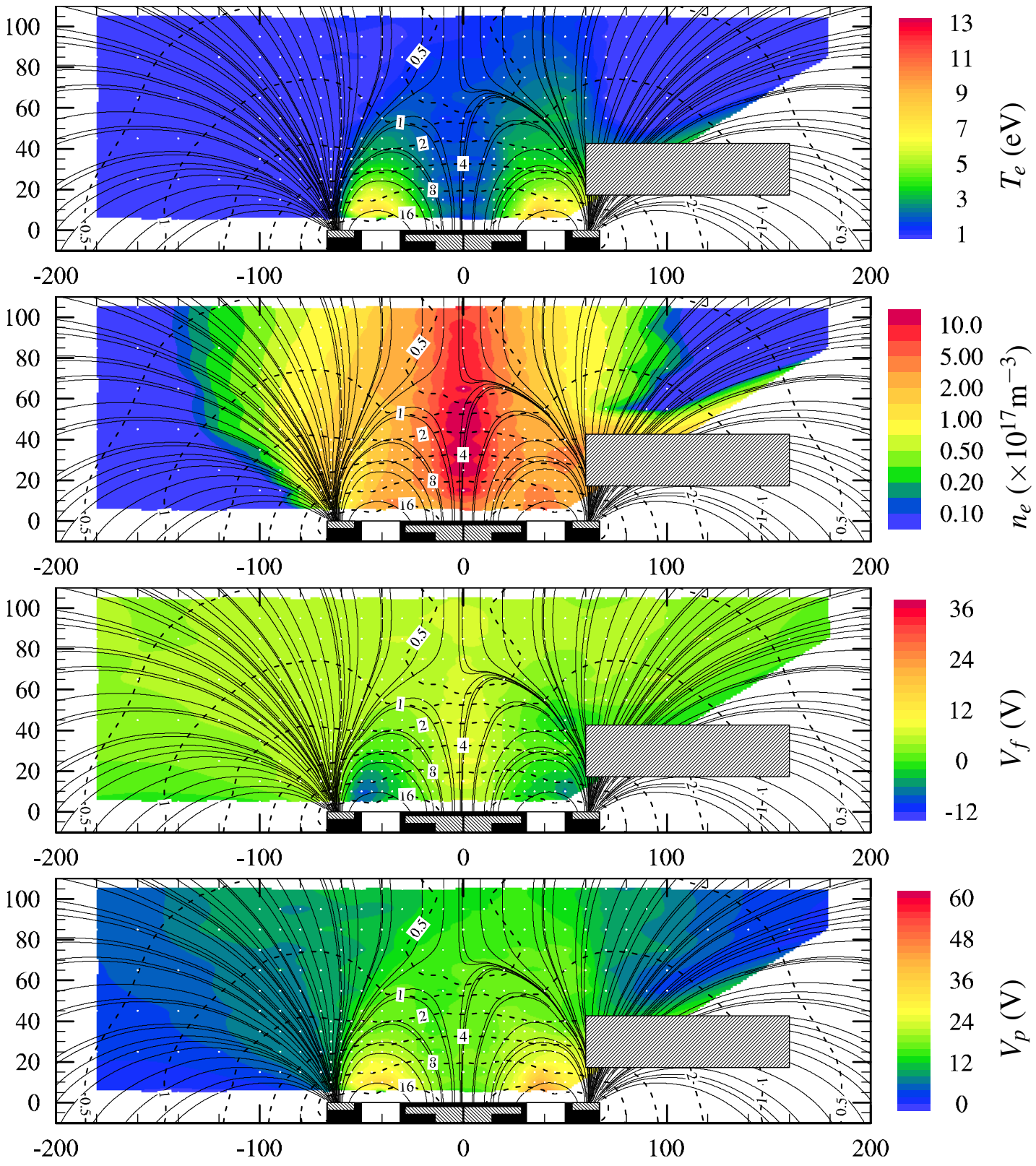

Figure 8.13: Plasma properties on the thruster with the OOP with $\dot{m}_{c}=10 \mathrm{SCCM}$ and the cathode at $r=60 \mathrm{~mm}$ 

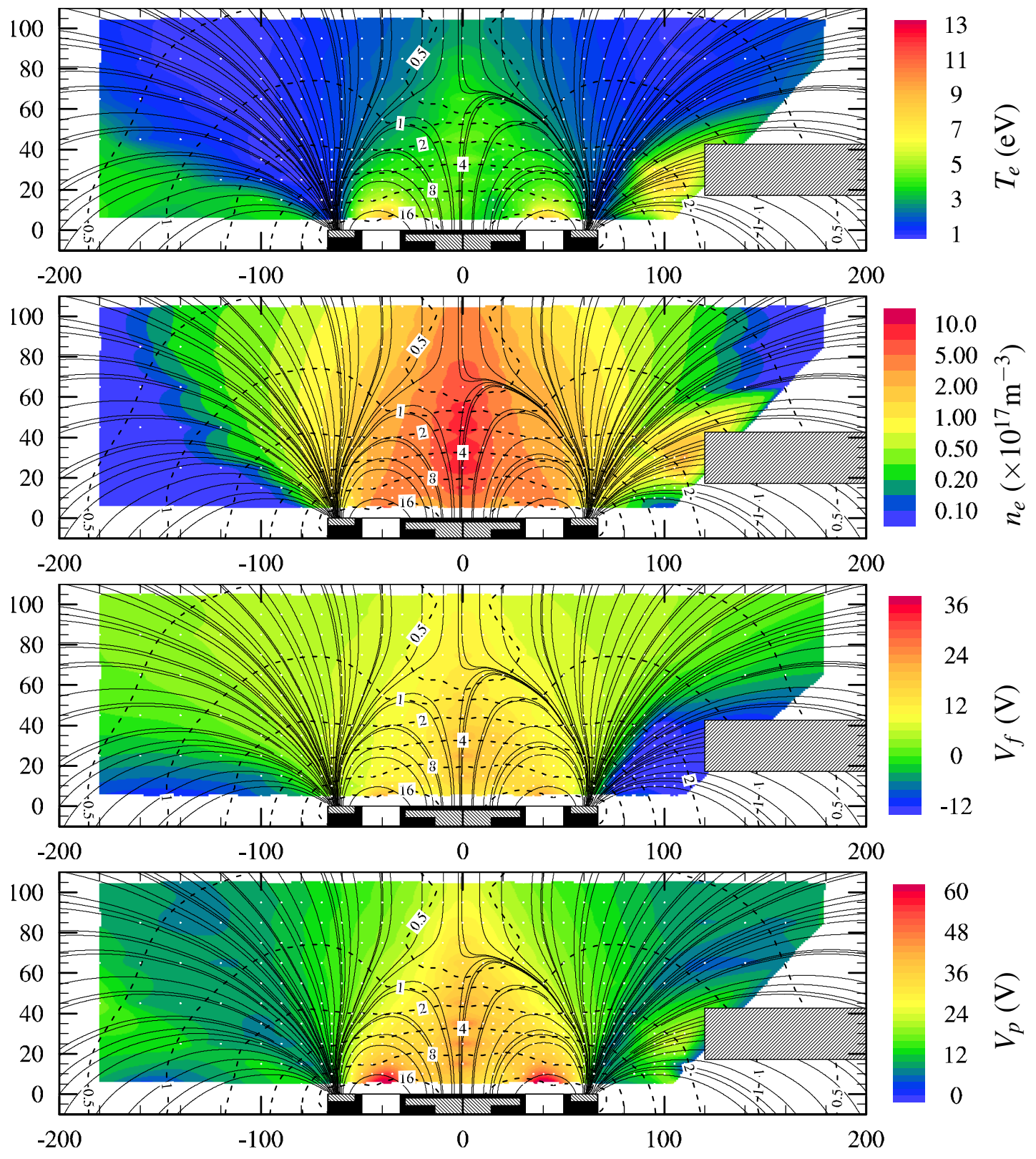

Figure 8.14: Plasma properties on the thruster with the OOP with $\dot{m}_{c}=10 \mathrm{SCCM}$ and the cathode at $r=120 \mathrm{~mm}$ 
Appendix B. An measure of the uncertainty can be made by examining the change in the measured parameters for a probe in a stationary position in a hot, dense region of the plume. The longest that the probe in the actual sweep pattern was exposed to dense, hot plasma was approximately one minute. Double probe traces were taken with the probe at $r=0 \mathrm{~mm}, z=10 \mathrm{~mm}$ at one sweep per second for 60 seconds. The standard deviations of the resulting analyses of $T_{e}$ and $n_{e}$ were $6 \%$ and $15 \%$ respectively. The standard deviation in $V_{f}$ was a negligible $1 \%$. The standard deviation in $V_{p}$ was $5 \%$. Propagation of the uncertainty on $T_{e}$ and $V_{f}$ through Equation 5.11 provides another estimate of the uncertainty on $V_{p}$, and yields $6 \%$. An additional systematic source of error in the measurement of $n_{e}$ is in the measurement of the area of the probes. The diameter of the probe is well established as it is manufactured to a tolerance of $2 \%$. However, the exposed length of the probe was measured by hand using calipers and was not more accurate than $0.5 \mathrm{~mm}$ on length measuring $4 \mathrm{~mm}$. This adds an additional error of about $12.7 \%$ arising from the uncertainty in probe area to the estimate of the uncertainty on the density. Adding the uncertainties in quadrature, the uncertainties estimate on $n_{e}$ is $20 \%$.

The reliability of the measurement is further complicated by the assumption of a Maxwellian plasma in the Peterson and Talbot theory. If the plasma is not sufficiently Maxwellian, (or if the data is simply too noisy) the measured probe traces will not be well fit by the theory. This is quantified by the cost function, Equation 5.29, which is plotted for all cases in Appendix C. A high cost fit will most strongly affect the measurement of $T_{e}$. For traces with costs near .1, which are in good agreement with the theory, the $6 \%$ estimate on errors in $T_{e}$ holds. For higher cost estimates, closer to .3 , the uncertainty may rise as high as $30 \%$ with a correspondingly higher uncertainty on $V_{p}$.

\subsubsection{Discussion}

Several features of the plume properties common to most of the operating conditions can be seen in the Figures 8.13 and 8.14. First one sees that $T_{e}, V_{f}$, and $V_{p}$ are both lower everywhere, and particularly in the near-field, when the cathode is placed at $60 \mathrm{~mm}$ as compared to when it is at $120 \mathrm{~mm}$. Meanwhile the electron density remains relatively constant, perhaps becoming slightly more divergent and less dense in the $120 \mathrm{~mm}$ case. Figure 8.15 shows, for example, the monotonic increase in plasma potential for every cathode position measured with the thruster operating with the EOP and $\dot{m}_{c}=10$ SCCM. Inspection of the plots in Appendix C reveals this same trend in all cases, as well as the trends in the other parameters. In Figure 8.15, one notes the particular increase in near-field potential between when the cathode is positioned essentially on the separatrix at $80 \mathrm{~mm}$ and when it is outside the separatrix at $100 \mathrm{~mm}$. This will be further explored in Section 8.6.3. 
By comparing any of the plots of Figure 8.15 to the plasma potential plots in either Figure 8.13 or Figure 8.14, one can see the plasma containment effect of the separatrix. The hot, high potential plasma is trapped within the separatrix. Note that near $z=$ $10 \mathrm{~mm}$ this higher potential plasma does not cross beyond $|r|=60 \mathrm{~mm}$. Outside the separatrix cooler, lower potential plasma prevails. Investigating the plasma potential plots in Figure 8.15 one notes that it is still when the cathode is at the separatrix, now located closer to $|r|=80 \mathrm{~mm}$ (for $z=10 \mathrm{~mm}$ ), that the division between those cathode positions with higher plume plasma potentials and those with lower potentials occurs.

In addition to the containment, the effect of the azimuthal drift arising from the Hall current may also be visible. In Figure 8.14 one notes the high electron temperature and high plasma potential just outside the orifice of the cathode and a corresponding region of low floating potential. On the opposite side of the figure, roughly in the region, $-200 \mathrm{~mm}<r<-100 \mathrm{~mm}$ and $0<z<30 \mathrm{~mm}$, a similar elevation in $T_{E}$ and $V_{p}$ and depression in $V_{f}$ can be seen. This effect can also be seen in most of the floating potential plots of Figure 8.16 and, of course, in numerous plots in Appendix C. In fact, there is also evidence of this reflection of the cathode plasma inside the separatrix when the cathode is positioned in or near the separatrix. This can be seen in the depression in $V_{f}$ on both positive and negative $r$ positions in Figure 8.16 when the cathode is at $r=50 \mathrm{~mm}$ and $r=60 \mathrm{~mm}$. The effect is even more striking in the numerous floating potential maps in Appendix $\mathrm{C}$ where the cathode is positioned at $r=50 \mathrm{~mm}$ (see Figures C.1, C.5, C.11, C.17, C.22, and C.28). The most reasonable conclusion is that the cathode plasma is "smeared out" around the periphery of the thruster, because of the tendency of electrons to drift in the $\mathbf{E} \times \mathbf{B}$ direction. This smearing, however, is not perfect and can be seen as an asymmetry in plasma parameters for cathode locations greater than $100 \mathrm{~mm}$. When the cathode is positioned in or near the separatrix, this asymmetry is much less noticeable.

One of the most striking features of the plume plasma is the formation of a double layer $^{7,8}$ between the cathode and the anode along magnetic field lines. This can be seen in the trapping of the hot, high-potential plasma from the cathode, apparently along magnetic field lines, as seen in Figure 8.14. Again, the floating potential shows the effect most clearly, owing to the low uncertainties in the data. Figure 8.16 shows the floating potential maps for each of the cathode positions when the thruster was operated with $\dot{m}_{c}=10$ SCCM and the OOP. As the cathode is moved in from its farthest point, the sharp boundary between the cathode-region plasma and the anode-region plasma is seen to push closer and closer to the separatrix, always falling along the magnetic field lines. After crossing the separatrix, a depression in potential appears to be confined on the field lines within the separatrix. The same behavior was seen, to one extent or another, in most of the plume data. The floating potential shows this feature more clearly because of the low uncertainty as compared to any of the remainder of the plume properties. Further inspection of the full data in Appendix $\mathrm{C}$ suggests that the 

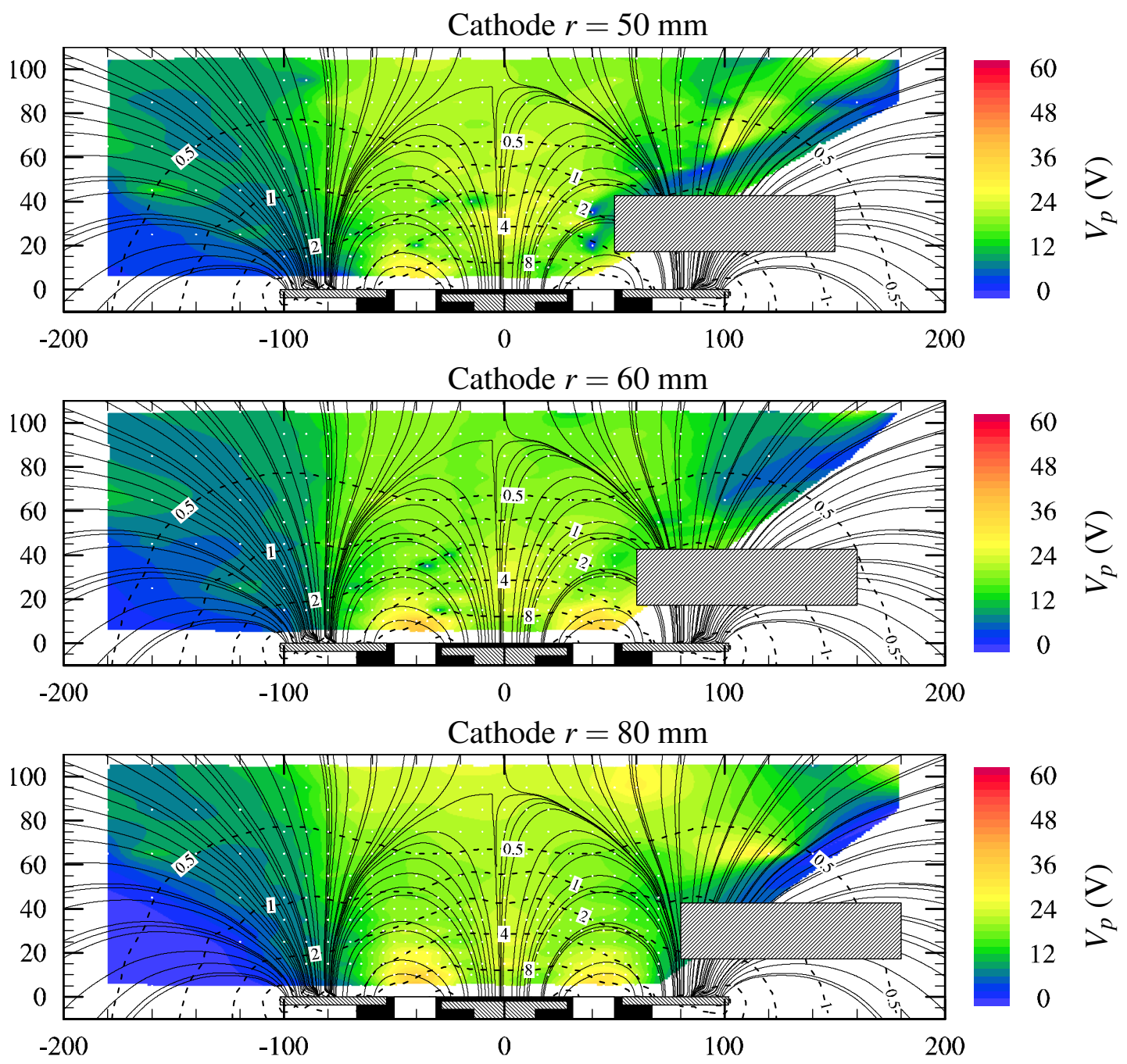

Figure 8.15: Plasma potential plots for the thruster operating with the EOP and $\dot{m}_{c}=10$ SCCM. The near-field plasma potential increases with cathode postion. 

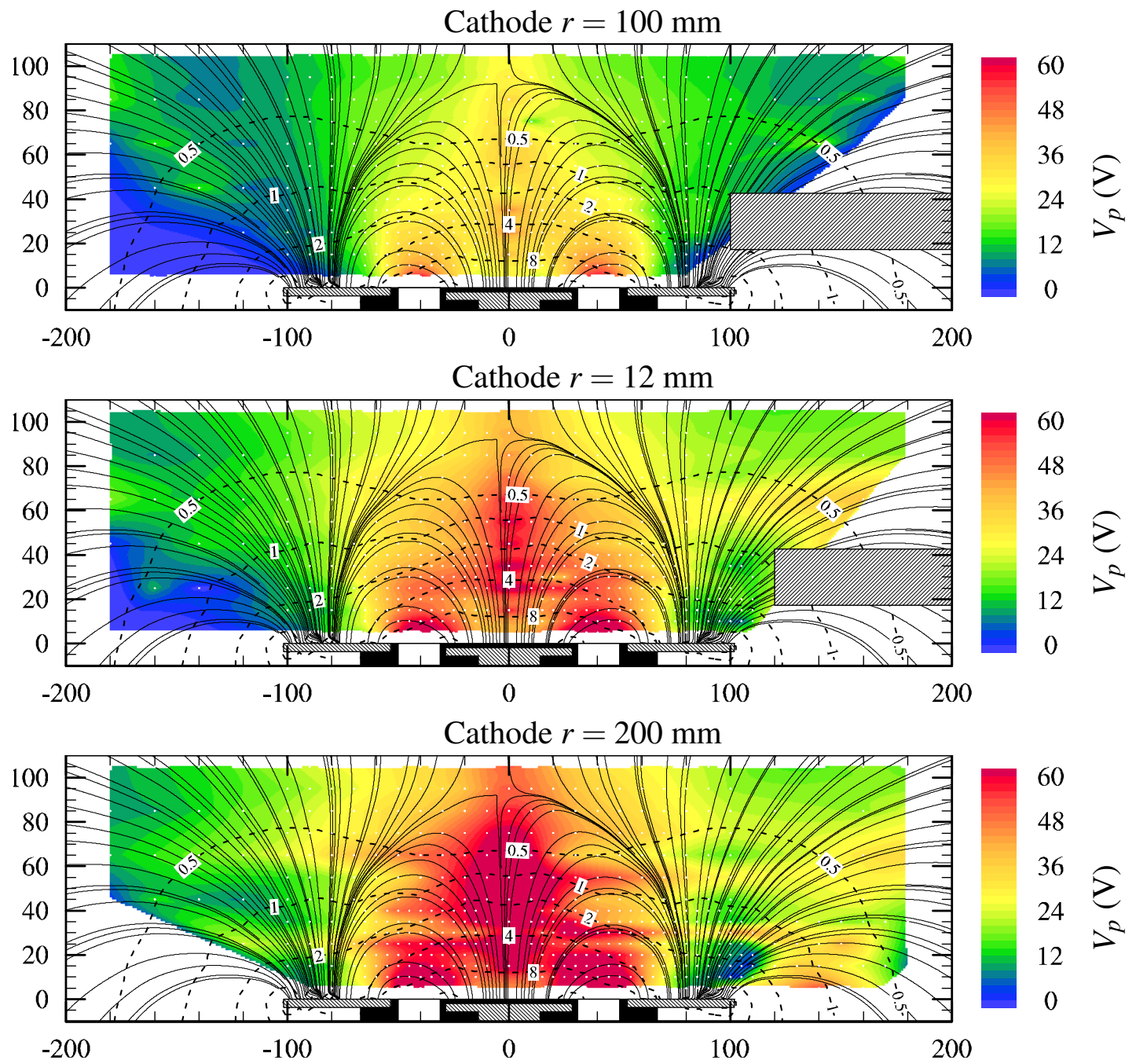

Figure 8.15: (continued) 
floating potential decreases drastically because of an increased electron temperature on the cathode side of the division, rather than a sudden drop in plasma potential.* None-the-less, the potential in the cathode region is lower than in the near-field plume, and often bordered by a distinct region of plasma that is lower still in potential, as in Figure 8.14. The existence of a double layer conforming to the magnetic field is a clear indication that the external field of the thruster is important in the cathode coupling process.

\subsubsection{Average near-field plume properties}

As a means of studying the trends in the variation of near-field plume plasma properties with cathode position, I have chosen to average the plasma properties in the near-field region to create a scalar value that can be easily compared. This raises the question, "What, exactly, is the near-field region?" Given the trapping effect of the separatrix discussed in the previous section, and the relatively similar properties seen inside the separatrix, I have chosen a hemi-ellipsoid centered on the origin and given by

$$
\left(\frac{r}{63 \mathrm{~mm}}\right)^{2}+\left(\frac{z}{70 \mathrm{~mm}}\right)^{2}<1, \quad z>0
$$

which approximates the separatrix for the original outer pole. While it may be reasonable to chose a similar region following the extended outer pole's separatrix in order to define the near-field plume of that configuration, I chose instead to use the same region for the EOP data, thereby maintaining the same number of data points and spatial extent. This was done to improve comparisons between OOP and EOP data. To perform the average, the data points were interpolated onto a $5 \mathrm{~mm} \times 5 \mathrm{~mm}$ grid, which is the size of the minimum grid spacing used for these data. The grid values at each grid vertex were then weighted by their corresponding cylindrical volume element and the total averages and standard deviations computed. For a given property $x\left(T_{e}, n_{e}\right.$, etc.), the average, $\bar{x}$, is computed by

${ }^{*}$ For two regions of plasma with the same $V_{p}$ and $n_{e}$, the one with a higher $T_{e}$ will exhibit a lower $V_{f}$, cf. Equation 5.11. 


$$
\begin{gathered}
z_{\max }=70 \mathrm{~mm} \\
r_{\max }(z)=63 \mathrm{~mm} \sqrt{1-\left(\frac{z}{70 \mathrm{~mm}}\right)^{2}} \\
D=\int_{0}^{z_{\max }} \int_{-r_{\max }}^{r_{\max }} r d r d z \\
\bar{x}=\frac{1}{D} \int_{0}^{z_{\max }} \int_{-r_{\max }}^{r_{\max }} x r d r d z,
\end{gathered}
$$

and the standard deviation $\sigma_{x}$ is given by

$$
\begin{gathered}
\left\langle x^{2}\right\rangle=\frac{1}{D} \int_{0}^{z_{\max }} \int_{-r_{\max }}^{r_{\max }} x^{2} r d r d z \\
\sigma_{x}=\sqrt{\left\langle x^{2}\right\rangle-\bar{x}^{2}} .
\end{gathered}
$$

Figure 8.17 presents the average plasma potential, floating potential, electron temperature, and electron density for the near-field region. The average provides a convenient way to look at broad trends in the data. These trends can also be seen by looking at the plume property maps in Appendix C. Figure 8.17a shows the data taken with the original outer pole, while Figure $8.17 \mathrm{~b}$ shows the data for the extended outer pole. The dashed line shows the radial location of the separatrix at the cathode axial position of $30 \mathrm{~mm}$. The error bars represent the standard deviation of the averaged points.

Studying Figure 8.17, one notices a general upward trend in both potentials and electron temperature as the cathode is moved farther away from the thrust axis and as $\dot{m}_{c}$ is reduced. This change in potential and $V_{\text {cg }}$ is reminiscent of the work on gridded ion thruster performed by Ward and King ${ }^{9}$ (see Chapter 3). Similar changes in potential have also been noted by Smirnov and Raitses while driving the cathode with "extra" heater power. ${ }^{10}$ Electron density remains generally flat, perhaps decaying slightly. Note that the plasma potential generally mirrors the cathode coupling potential-as $V_{\text {cg }}$ decreases, $V_{p}$ increases such that the potential difference between the two points increases all the more. Interestingly, a different trend was seen by Hofer, when working with trim coils. As discussed in Section 3.3.3, Hofer noted an increase in both floating potential (presumably implying an increase in plasma potential) and $V_{\mathrm{cg}}$ rather than opposing trends. Of course, in those experiments the cathode was not repositioned. Instead, the magnetic field structure was varied, which may have affected the coupling in a different way than was seen in the present experiments.

The increase in external plasma potential is to be expected if the magnetic field is significantly impeding the progress of electrons from the cathode to the beam and an- 

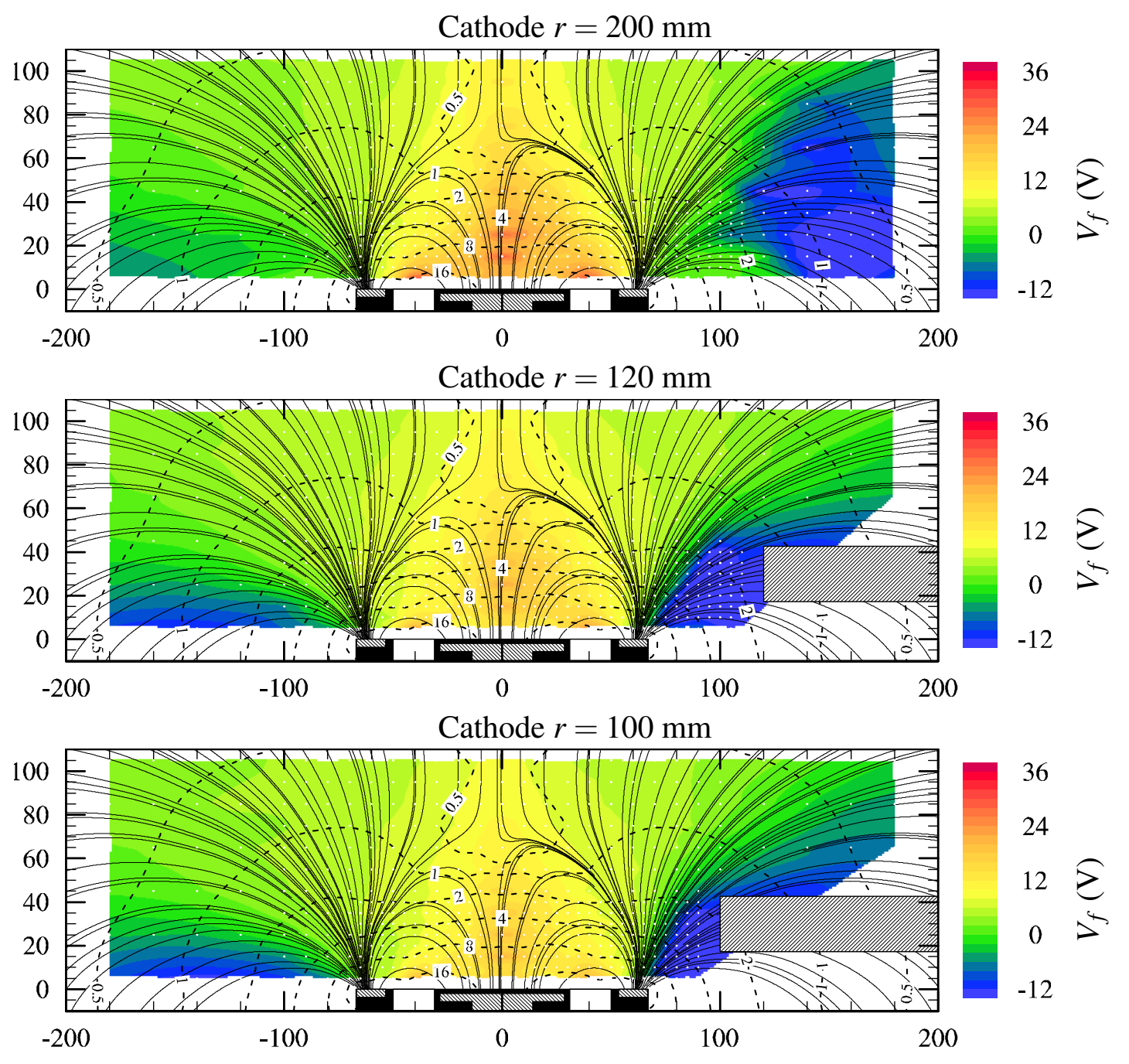

Figure 8.16: Floating potential maps exhibit a double-layer between the cathode and beam plasmas 

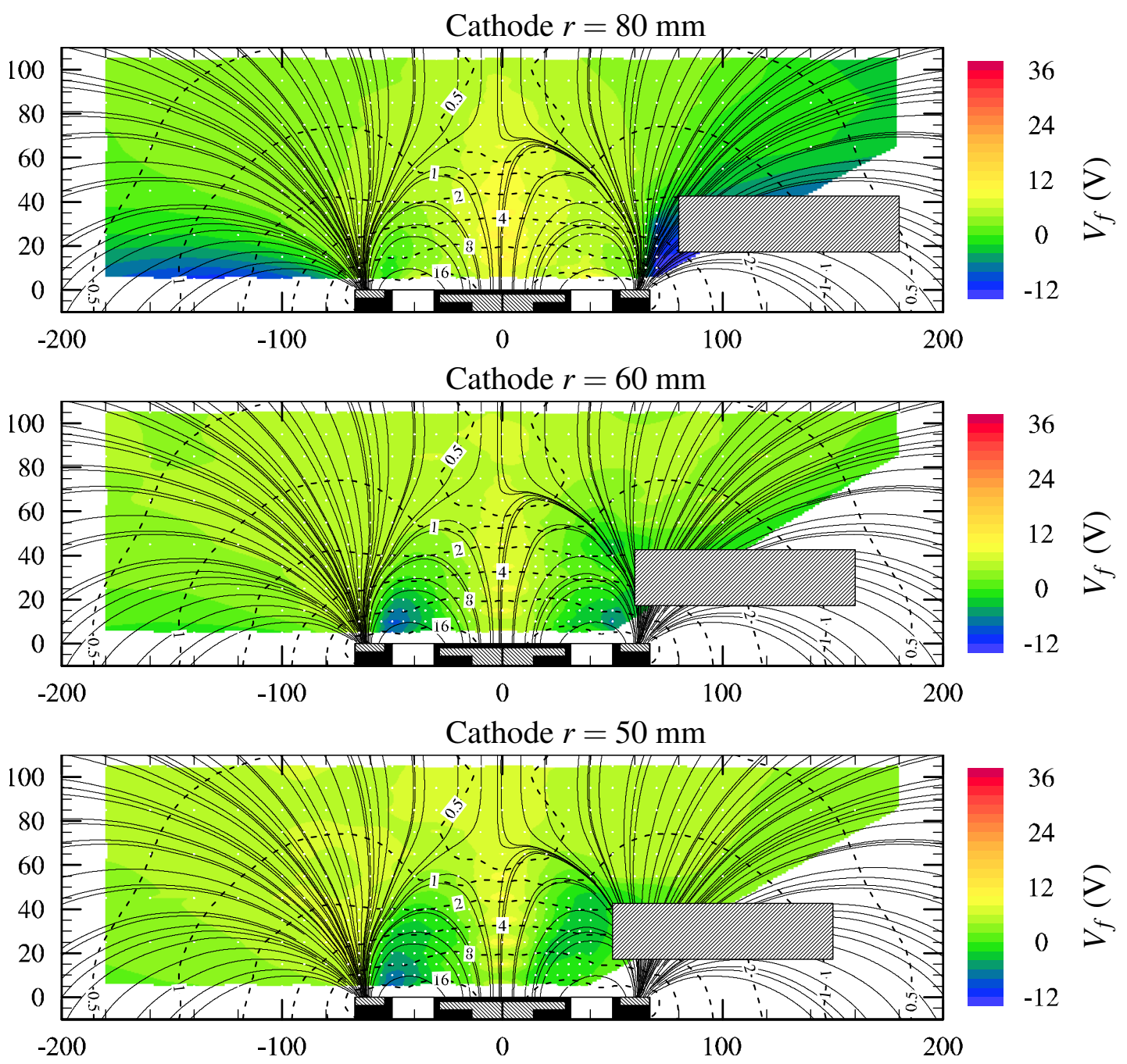

Figure 8.16: Floating potential maps exhibit a double-layer between the cathode and beam plasmas (continued) 

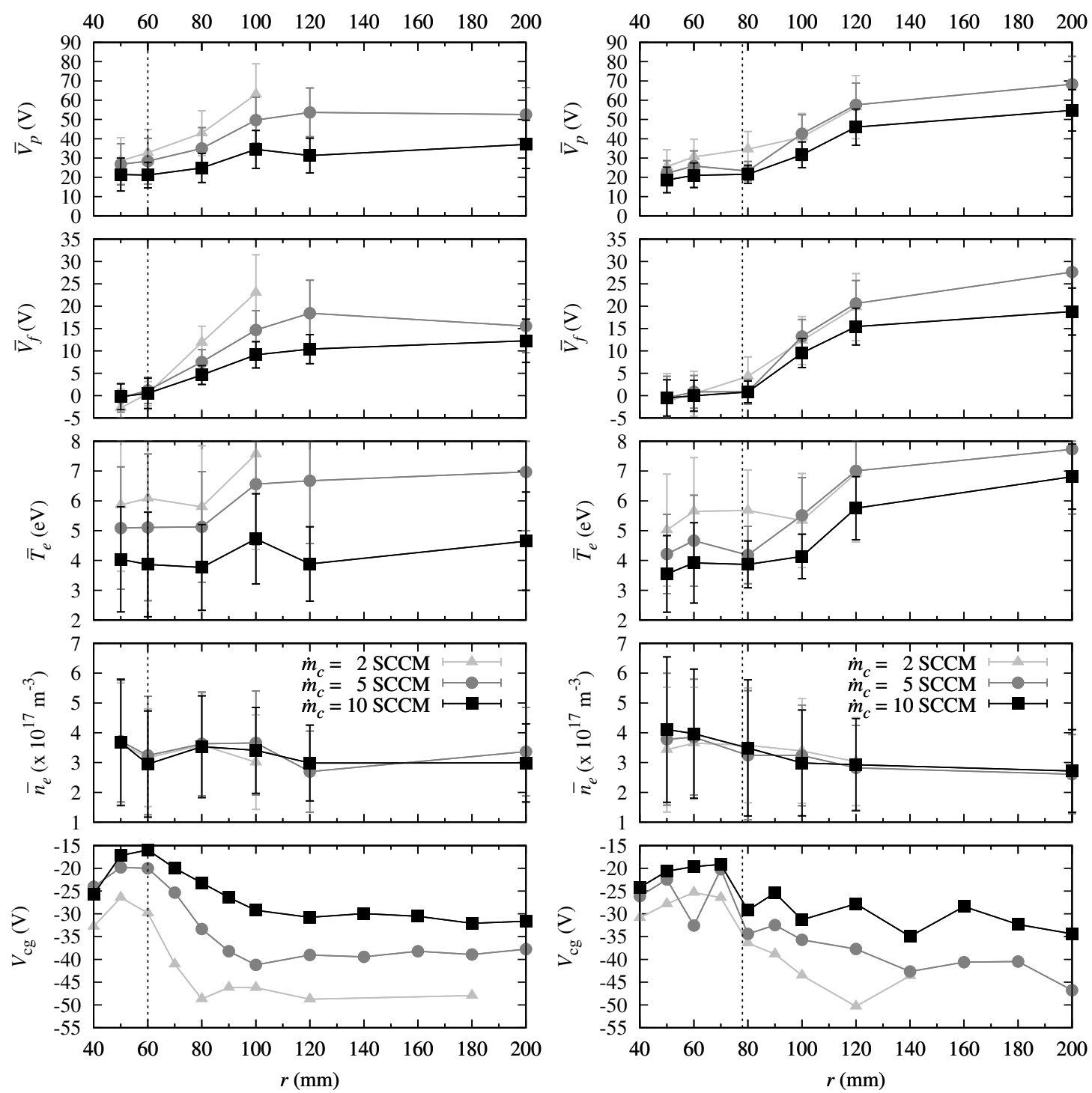

(a) OOP

(b) EOP

Figure 8.17: Average near-field plume properties as a function of cathode position. Error bars represent standard deviations of the averaged quantities. 
ode. Placing the cathode at further radial distances induces a greater resistance, as the electrons must traverse a greater distance across the magnetic field. The potential must increase to compensate for the increased resistance. Most interestingly, the variation of both plasma potential and the cathode coupling voltages with cathode position are relatively flat inside the separatrix, excluding $V_{\mathrm{cg}}$ at $40 \mathrm{~mm}$, at which point the cathode physically blocked the exit channel. Only when the cathode is moved outside the separatrix does the near-field plasma potential increase and the cathode coupling voltage decrease significantly. This suggests improved coupling when the cathode is within the separatrix. Phenomenologically, this is reasonable, since electrons bound to magnetic field lines outside the separatrix are directed further downstream and ultimately away from the beam, while those on internal field lines are directed immediately to the beam.

The rate at which the potentials, both $V_{p}$ and $V_{\text {cg }}$, change with cathode position as the cathode is moved away from the separatrix increases with cathode mass flow rate. This suggests that there is improved mobility due to collisions between electrons and neutrals - that is, an increased classical mobility component-which is to be expected at higher cathode flow rates. Again, this effect has been repeatedly noticed $9,11,12,13,14$ (see Section 3.3.2).

The increased near-field plume plasma potential has the effect of increasing ion beam divergence. The correlation between the two can be seen in Figure 8.18, which shows the average near-field plume plasma potential (as in Figure 8.17) overlaid with the beam divergence efficiencies from Figure 8.12. Note that the efficiencies are plotted on a reversed y-axis. The correlation between the two processes is due to the fact that the electric field immediately external to the thruster is largely radial. The greater the potential in the near field, the higher the diverging force on the ions. As an example, Figure 8.19 shows the direction of the electric field calculated by taking the gradient of the plasma potential for the $60 \mathrm{~mm}$ and $120 \mathrm{~mm}$ cathode positions using the OOP. One notes the generally stronger radial components in the $120 \mathrm{~mm}$ case.

The increased external plasma potential may also explain the general trend of the both voltage utilization and velocity distribution efficiencies increasing with increasing cathode position beyond $60 \mathrm{~mm}$. As the the near-field plume reaches higher potentials, it is likely that the higher potential regions inside the channel are also pulled further downstream. This gives ions created further downstream greater energy, thus increasing $\eta_{V}{ }^{*}$ If the primary ionization region is not drawn down stream as far as the high potential region, then there would be a corresponding decrease in population of the slower moving ions. That would result in a decreased spread in velocity space, thereby improving $\eta_{\mathrm{vdf}}$. However, without internal measurements of the HET it is impossible to confirm this speculation.

*That is, by the time they reach the tank wall or the RPA used to measure ion energy, the ions have more energy than those created in a lower potential region. 


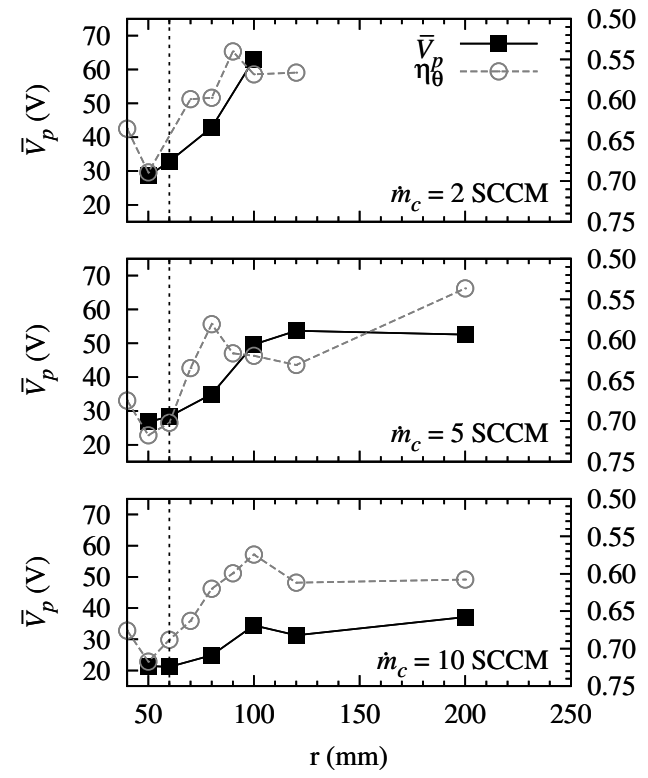

(a) Original outer pole

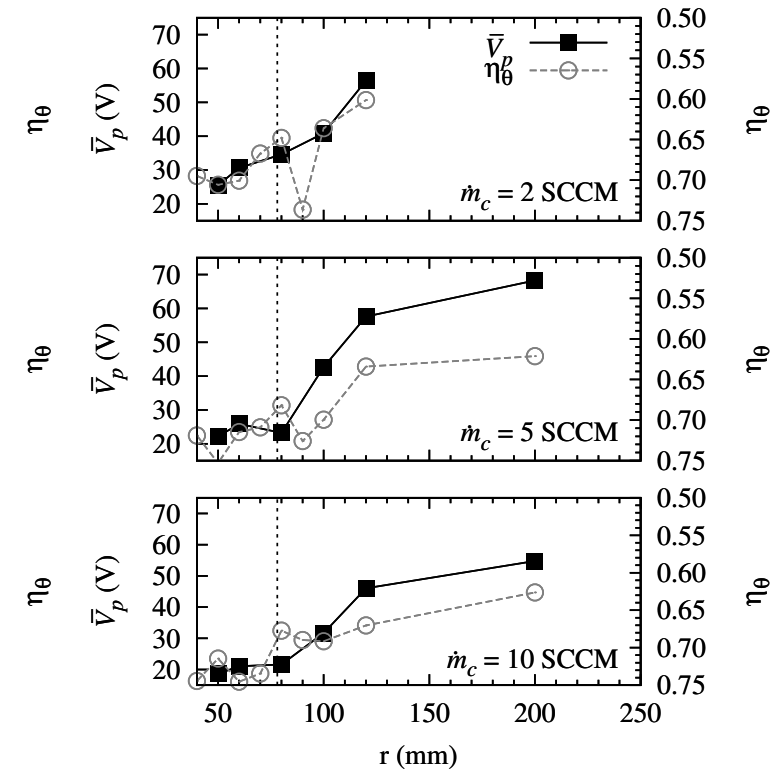

(b) Extended outer pole

Figure 8.18: Comparison of the average near-field plasma potential to the beam divergence efficiency

\subsection{Discussion}

A considerable amount of data were collected in this experiment, and it is difficult to get a complete picture from any individual measurement. To facilitate the synthesis of all of the data, I have compiled a table of all of the scalar results for each operating condition. It lists each operation point - that is, combination of outer pole type, cathode mass flow, and cathode radial position - combined with the corresponding scalar measurements and calculations, e.g. thrust, beam divergence, average near-field plasma potential, efficiency components, total efficiency, etc. The table is found in Appendix E. I then calculated the linear, Pearson product-moment correlation coefficient for each combination of parameters.

For two variables, $a$ and $b$ sampled from a population with estimated variances of $s_{a}^{2}$ and $s_{b}^{2}$, the correlation coefficient is given by

$$
r=\frac{1}{s_{a} s_{b}} \frac{1}{N-1} \sum_{i}^{N}\left(a_{i}-\bar{a}\right)\left(b_{i}-\bar{b}\right) .
$$

Here, $\bar{a}$ and $\bar{b}$ represent the estimated means. In these data, the sampled data $a$ and $b$ do not necessarily have the same number of datapoints in them. For instance, performance 


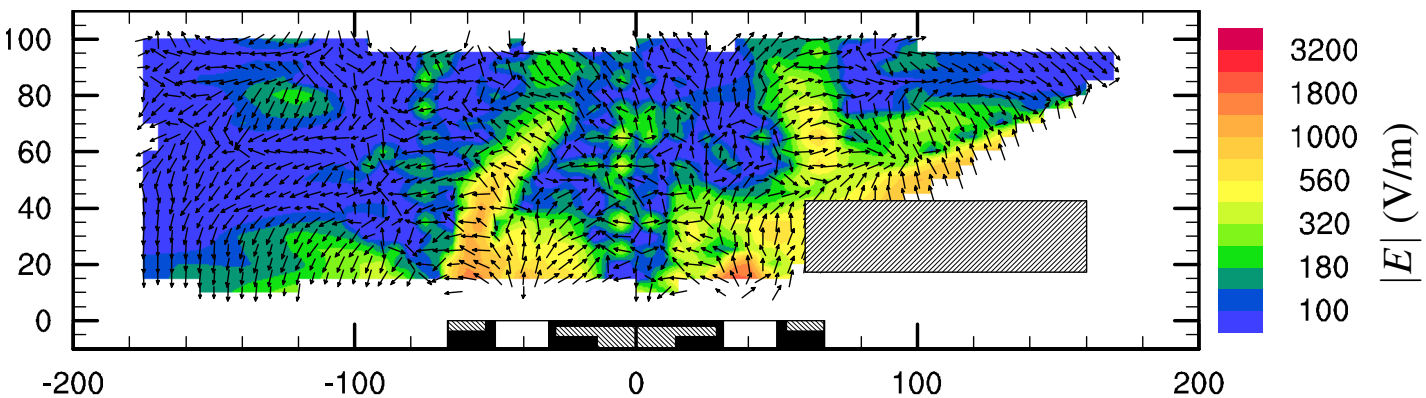

(a) $r=60 \mathrm{~mm}$

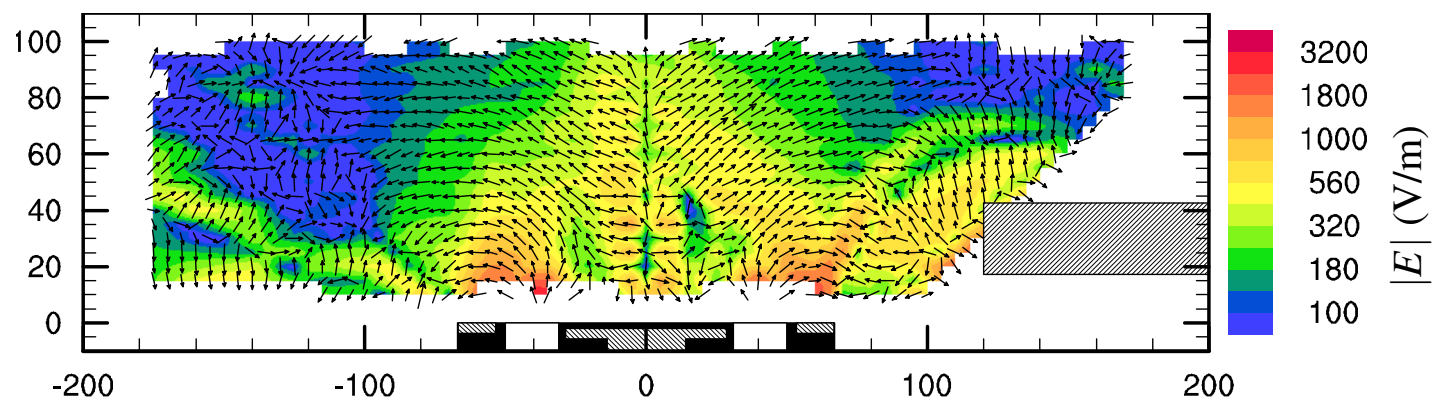

(b) $r=120 \mathrm{~mm}$

Figure 8.19: The electric field for $\dot{m}_{c}=10 \mathrm{SCCM}$ with the cathode at 60 and $120 \mathrm{~mm}$ on the OOP configuration. The magnitude of the field is plotted in a log scale on the color map. The arrows show the direction of the field.

data was taken at 13 cathode positions, $r$, between $40 \mathrm{~mm}$ and $250 \mathrm{~mm}$ for each outer pole and $\dot{m}_{c}$, while plume properties were measured at only 6 cathode positions for each outer pole and $\dot{m}_{c}$. This problem is resolved as follows. If $A$ is the set of operation conditions for which variable $a$ is available, and $B$ is the set of operating conditions for which $B$ is available, and $N_{A}$ and $N_{B}$ are the number of items in $A$ and $B$, respectively, then 


$$
\begin{aligned}
\bar{a} & =\frac{1}{N_{A}} \sum_{i \text { in } A} a_{i} \\
s_{a}^{2} & =\frac{1}{N_{A}-1} \sum_{i \text { in } A}\left(a_{i}-\bar{a}\right)^{2} \\
\bar{b} & =\frac{1}{N_{B}} \sum_{i \text { in } B} b_{i} \\
s_{b}^{2} & =\frac{1}{N_{B}-1} \sum_{i \text { in } B}\left(b_{i}-\bar{b}\right)^{2} \\
C & =A \cap B \\
r & =\frac{1}{s_{a} s_{b}} \frac{1}{N_{C}-1} \sum_{i \text { in } C}\left(a_{i}-\bar{a}\right)\left(b_{i}-\bar{b}\right) .
\end{aligned}
$$

It should be noted that this formulation does not guarantee that $r \leq 1$. It is possible for it to be slightly greater in rare circumstances. However, this formulation maintains the best estimates of the averages and variances of the independent datasets, only reducing the data used in the calculation when absolutely necessary.

There are two caveats with the broad application of this method in this case. Most importantly, the correlation coefficient represents the degree to which a linear relationship exists between two variables. A strong non-linear relationship may exist without there being any indication in $r$ if, for instance, the data was sinusoidal. However, so long as the relationship is monotonic, $r$ will typically show some indication of the strength of the correlation. Inspection of the correlation plot will reveal the quality and the nature of the relationship. The second caveat is that correlation does not imply causation. Unless we have reason to suspect there is a causal relationship between two variables, we cannot draw strong conclusions from a correlation. However, causation does imply correlation, though not necessarily a linear one. Therefore it is worth investigating correlated variables for an underlying causal link.

Table 8.2 shows the correlation coefficients calculated according to Equation 8.31. Each cell contains the correlation coefficient between the two variables found in the corresponding row and column headers. For convenience, the variables are grouped by related types. A few of the variables deserve some explanation, and it is provided below the table. The cell backgrounds are colored according to the value of $|r|$, with darker colors representing higher values. Thus, dark cells are highly correlated, while light cells are not. Negative values of $r$ simply indicate an inverse relationship between the two variables, i.e. as $a$ increases, $b$ decreases.

The best way to approach the table is to look first at the column and row headers, and understand how the table is laid out. Then, step back and look for cells that are particularly dark or particularly light. Some of the correlation coefficients are expected. For instance, there is a strong correlation between $\eta_{a}$ and $\eta_{t}$ which is expected as they 


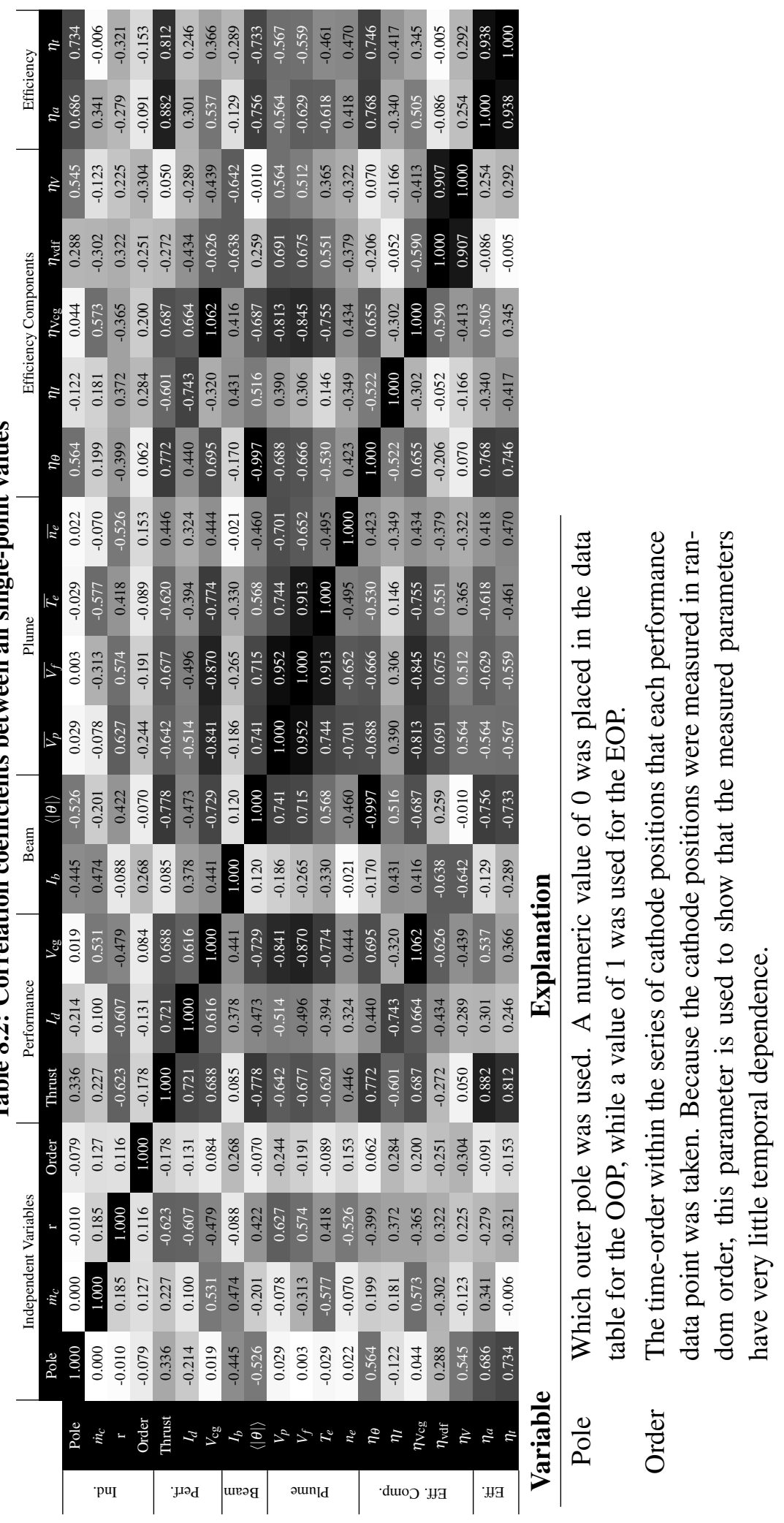


differ only by a small factor (see Equation 2.22 and Equation 2.23). Looking at the plume data, we see strong correlation between $\overline{V_{p}}$ and $\overline{T_{e}}, \overline{n_{e}}$, and $\overline{V_{f}}$. This is expected, as $V_{p}$ is calculated from $T_{e}, n_{e}$, and $V_{f}$ according to Equation 5.22. In fact, the only two plume variables which are not strongly correlated are $\overline{T_{e}}$ and $\overline{n_{e}}$, which are not mathematically related, and any correlation between them would be due to physical processes. It is good to see there is little correlation between the order in which data were taken and any of the variables, suggesting that the experiment was immune to any time-based drift errors.

There are a few correlations that we have already noted in a less rigorous manner. First of all, we have noted in Section 8.5 that $\eta_{\mathrm{vdf}}$ and $\eta_{V}$ vary together and discussed the expected reasons. The correlation coefficient for these two is 0.907 , and the correlation plot is shown in Figure 8.20a. A strong correlation between $\eta_{\theta}$ and $\eta_{\mathrm{Vcg}}$ was also noted, and this is shown in Figure $8.20 \mathrm{~b}$. The correlation coefficient for this pair is 0.655 . Section 8.6 noted the correlation between $\overline{V_{p}}$ and $\eta_{\theta}$ and $\overline{V_{p}}$ and $V_{\text {cg. There- }}$ fore, the correlation between $\overline{V_{p}}$ and $\eta_{\mathrm{Vcg}}$ is not surprising. The correlation values for $\overline{V_{p}}$ with $\eta_{\theta}$ and $\eta_{\mathrm{Vcg}}$ are -0.688 and -0.813 respectively. The corresponding diagrams are shown in Figures 8.20c and 8.20d, respectively. These correlations lead us to suspect that there is a physical mechanism by which certain cathode positions cause an increased potential difference between the cathode and the plume, and this results in increased beam divergence, as suggested by Figure 8.19, and lower thruster performance. This relationship will be further explored in the conclusion. 


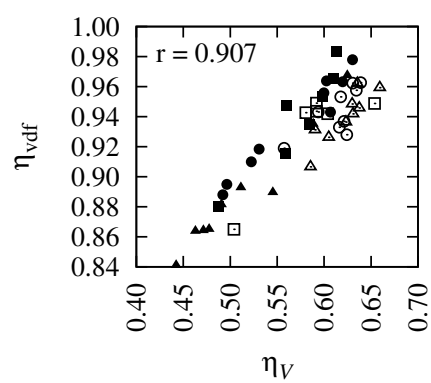

(a) $\eta_{\text {vdf }}$ vs. $\eta_{V}$

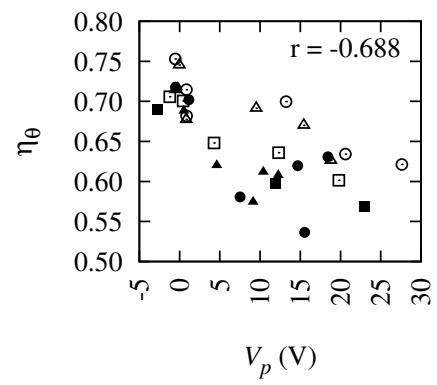

(c) $V_{p}$ vs. $\eta_{\theta}$

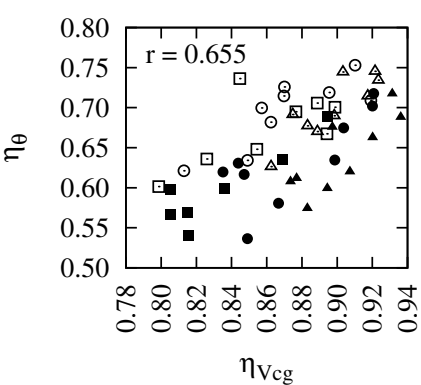

(b) $\eta_{\theta}$ vs. $\eta_{\mathrm{V} c \mathrm{~g}}$

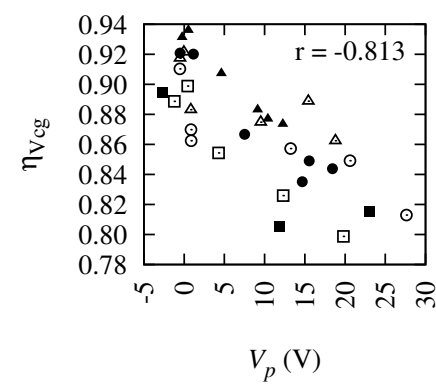

(d) $V_{p}$ vs. $\eta_{\mathrm{Vcg}}$

Figure 8.20: Correlation plots for select variables 


\section{References for Chapter 8}

[1] Linnell, J. A. and Gallimore, A. D., "Efficiency Analysis of a Hall Thruster Operating with Krypton and Xenon," Journal of Propulsion and Power, Vol. 22, No. 6, November 2006, pp. 1402-1412.

[2] Kim, V., Popov, G., Kozlov, V., Skrylnikov, A., and Grdlichko, D., "Investigation of SPT Performance and Particularities of it?s Operation with $\mathrm{Kr}$ and $\mathrm{Kr} / \mathrm{Xe}$ Mixtures," 27th International Electric Propulsion Conference, Pasadena, CA, October 15-19, 2001.

[3] Larson, C. W., Brown, D. L., and Hargus, W. A., "Thrust Efficiency, Energy Efficiency and the Role of VDF in Hall Thruster Performance Analysis," 43rd AIAA/ASME/SAE/ASEE Joint Propulsion Conference, Cincinnati, OH, July 8-11, 2007.

[4] Ross, J. L. and King, L. B., "Energy Efficiency in Low Voltage Hall Thrusters," 43rd AIAA/ASME/SAE/ASEE Joint Propulsion Conference, July 8-11, 2007, AIAA-2007-5179.

[5] Rovey, J. L., Walker, M. L. R., Gallimore, A. D., and Peterson, P. Y., "Magnetically filtered Faraday probe for measuring the ion current density profile of a Hall thruster," Review of Scientific Instruments, Vol. 77, No. 1, 2006, Paper No. 013503.

[6] Sibson, R., "A brief description of natural neighbor interpolation (Chapter 2)," Interpreting Multi-variate Data, edited by V. Barnett, John Wiley \& Sons, Chichester, 1981, pp. 21-36.

[7] Block, L. P., “A Double Layer Review," Astrophysics and Space Science, Vol. 55, No. 1, May 1978, pp. 59-83.

[8] Hershkowitz, N., "How Does the Potential Get From A to B in a Plasma?" IEEE Transactions on Plasma Science, Vol. 22, No. 1, February 1994, pp. 11-21.

[9] Ward, J. W. and King, H. J., "Mercury Hollow Cathode Plasma Bridge Neutralizers," Journal of Spacecraft and Rockets, Vol. 5, No. 10, October 1968, pp. 11611164.

[10] Smirnov, A. A. and Raitses, Y., "Controlling the Plasma Flow in the Miniaturized Cylindrical Hall Thruster," IEEE Transactions on Plasma Science, Vol. 36, No. 5, October 2008, pp. 1998-2003. 
[11] Tilley, D. L., de Grys, K. H., and Myers, R. M., "Hall thruster-cathode coupling," 35th AIAA/ASME/SAE/ASEE Joint Propulsion Conference, June 20-24, 1999, Paper No. AIAA-99-2865.

[12] Albarède, L., Lago, V., Lasgorceix, P., Dudeck, M., Burgova, A., and Malik, K., "Interaction of a Hollow Cathode Stream with a Hall Thruster," 28th International Electric Propulsion Conference, Toulouse, France, March 17-21, 2003, Paper No. 03-333.

[13] Manzella, D. H., Jankovsky, R. S., and Hofer, R. R., "A Laboratory Model 50kW Hall thruster," 38th AIAA/ASME/SAE/ASEE Joint Propulsion Conference, Indianapolis, IN, July 7-10, 2002, Paper No. AIAA-2002-3676.

[14] Manzella, D. H., Jacobson, D., and Jankovsky, R. S., "High Voltage SPT Performance," 37th AIAA/ASME/SAE/ASEE Joint Propulsion Conference, Salt Lake City, UT, July 8-11, 2001, Paper No. AIAA-2001-3774. (Also NASA TM-200121135.) 


\section{Chapter 9}

\section{Extended Outer Pole Performance}

\subsection{Motivation and Design of Experiment}

The original outer pole (OOP) and the extended outer pole (EOP) separatrix crossing experiments were conducted with differing magnet currents causing differing magnetic field strengths. Therefore, direct comparison between the OOP and EOP runs of the performance data is not strictly justified. Therefore the following test was designed. The cathode was positioned at $z=30 \mathrm{~mm}, r=70 \mathrm{~mm}$, roughly halfway between the separatrix on the OOP and that of the EOP at $z=30 \mathrm{~mm}$ (see Figure 8.2c for a comparison of the fields). In this position, the thruster was operated in the standard fashion with the EOP mounted. As in the prior experiments, the thruster was operated at $V_{d}=250 \mathrm{~V}$ and $\dot{m}=41 \mathrm{SCCM}$ (the molar equivalent of $4 \mathrm{mg} / \mathrm{s}$ of xenon). As with the separatrix crossing experiments, the propellent was krypton.

Once the thruster had reached steady-state operation, the magnet current was varied over a range of values which included the optimal value of $\sim 1.3 \mathrm{~A}$. The cathode mass flow rate was also varied between the three values used in the prior experiment: 2, 5, and 10 SCCM. The thrust, discharge current, cathode coupling voltage and efficiency, along with the rest of the standard telemetry data were measured in the usual way (see Section 4.3).

After this experiment was performed, the EOP was replaced with the OOP, which required bringing the vacuum chamber up to atmosphere. After switching the outer poles, the chamber was re-evacuated and the experiment repeated.

\subsection{Results}

Figure 9.1 shows the results of the experiment as a function of magnet current. The first and second graphs from the top show total and anode efficiencies, respectively. The third and fourth graphs show the measurements of thrust and discharge current from 


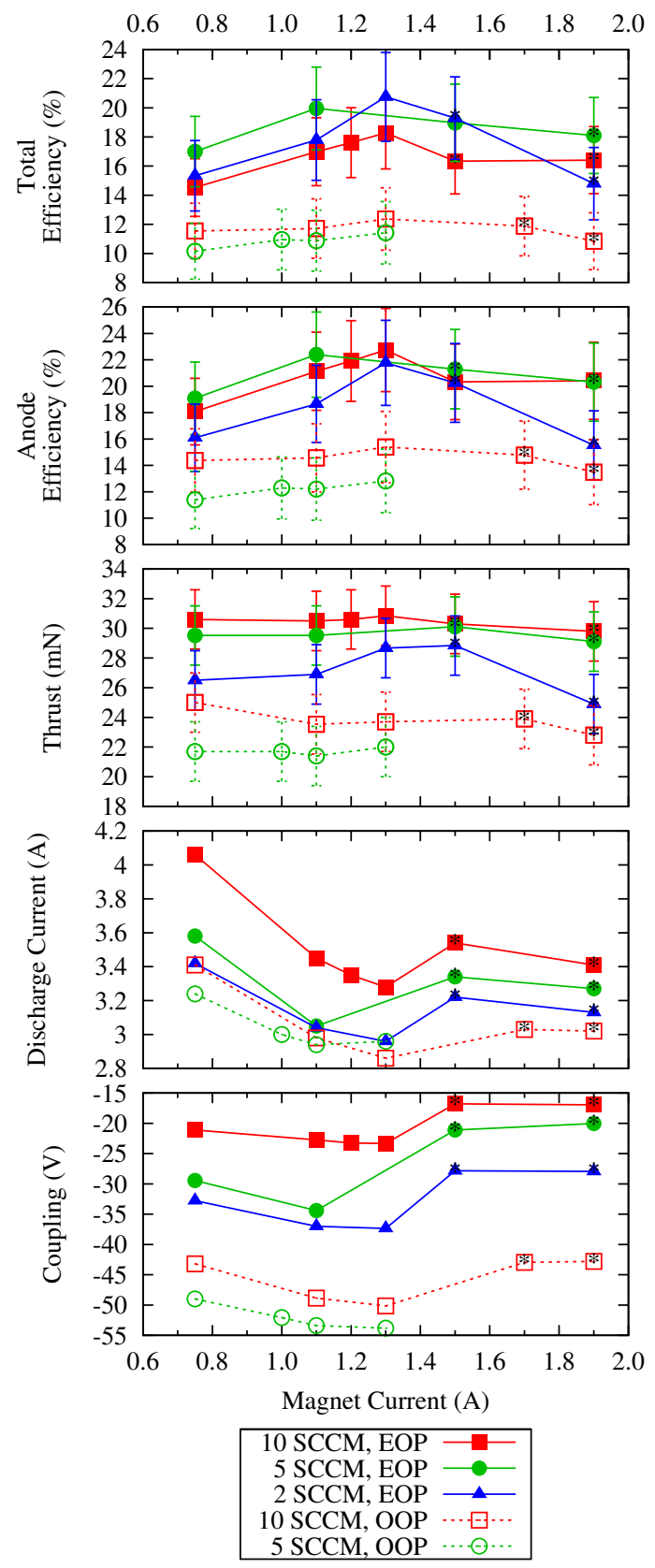

Figure 9.1: Direct comparison of thruster performance when running with the OOP and EOP at identical operating conditions 
which the efficiencies are derived according to Equation 2.22 and Equation 2.23. The bottom graph shows the cathode coupling voltages. Differing marker colors denote the different cathode mass flow rates. Solid markers and lines denote data from the EOP, while open markers and dashed lines denote data from the OOP. With the OOP, the thruster would not operate stably at the 2 SCCM cathode flow rate, nor would it operate at any $I_{\text {mag }}$ greater than $1.3 \mathrm{~A}$ when $\dot{m}_{c}$ was 5 SCCM.

The errors are estimated as in Section 8.3.1, with the error on the thrust measurements estimated at $2 \mathrm{mN}$. This yields errors in the efficiencies of about 2.5 percentage points.

\subsection{Discussion}

It is obvious from Figure 9.1 that the EOP did indeed improve the efficiency of the thruster. The figure shows that the efficiency with the EOP is five to ten percentage points greater than the efficiency with the OOP. The anode efficiency, for instance, increases from approximately $13 \%$ (on average) for the OOP to approximately $22 \%$. Furthermore, the change is consistently outside the range of the error bars, suggesting that the change is more than the result of statistical happenstance.

The only change between the two experiments is the magnetic field topology. Therefore, it is reasonable to attribute the improved performance of the EOP to the position of the cathode in the magnetic field topology. This interpretation is further supported by the fact that the cathode coupling voltages for the OOP data are $\sim 25 \mathrm{~V}$ lower than with the EOP, corresponding to coupling efficiencies of $\sim 80 \%$ for the OOP rather than $>90 \%$ for the EOP. Clearly the coupling is improved.

One could argue that internal rather than external magnetic field changes are responsible for the change in efficiency. As already mentioned, the simple process of placing a bigger outer pole did not control for these changes. While this is certainly possible, inspection of the internal field of the two designs, reveals small change in the internal field topology, as shown in Figure 9.2. The dotted lines in parts (a) and (b) show the line sections on the exit plane and channel center extracted for quantitative comparison. The large dots indicate 0 on the line-section axis, and the arrowheads indicate the direction of positive increase. To compare the vector fields, the magnitudes along the line sections of both fields are shown in the top plots of (c) and (d). The bottom plots of (c) and (d) show the difference in field direction between the two configurations. Along the exit plane and inside the channel both magnitude and angle agree well along both cross sections except very close to the poles, where the angle difference is great. Beyond the exit plane $\left(z_{c}>0\right)$ the angle of the field differs more widely. In any case, a more carefully designed EOP should strive for an optimized inner field structure while modifying the external structure to move the separatrix further away. 


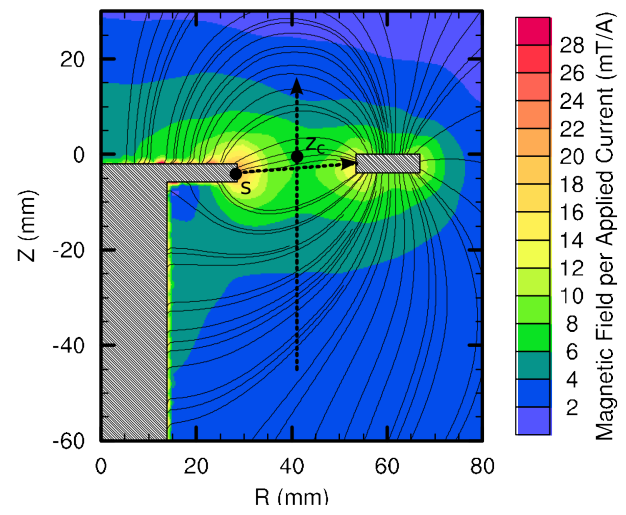

(a) OOP

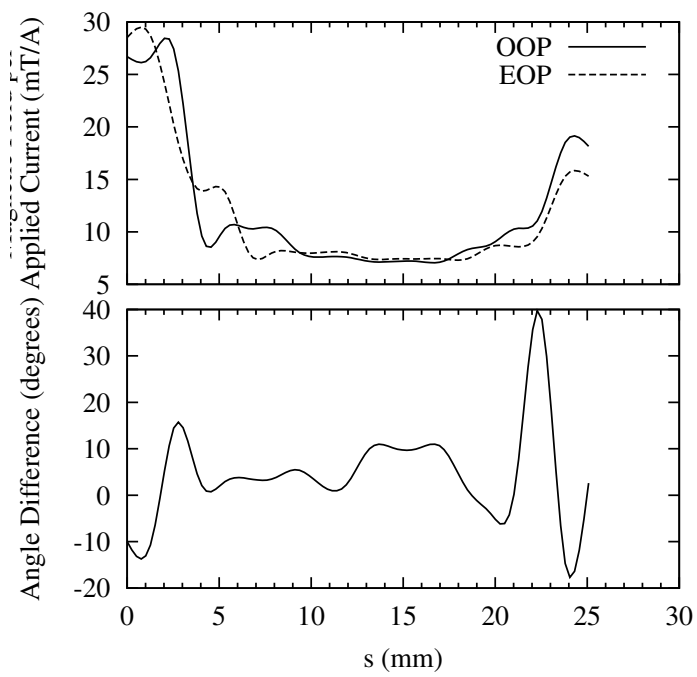

(c) Exit Plane Comparison

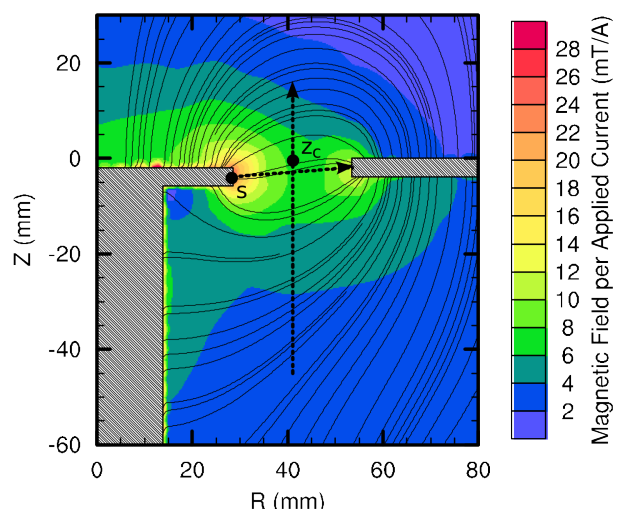

(b) EOP

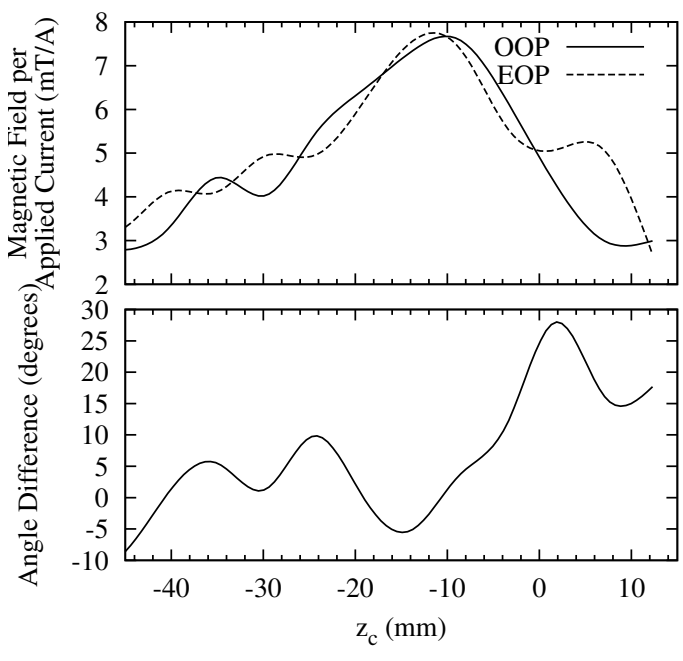

(d) Center Channel Comparison

Figure 9.2: Comparison of the internal magnetic fields of the thruster with the original and extended outer pole.

A second argument is that the extended face may simply be directing more cathode neutrals to the discharge region, thereby making better use of the cathode propellent. This is indeed likely for more radially distant cathode positions. However, the choice of $70 \mathrm{~mm}$ as the point at which to run these experiments nullifies this argument as the cathode tip is nearly even with the outer edge of the outer pole with the OOP, a gap of a mere $3 \mathrm{~mm}$ being present. Furthermore, if the thruster were ingesting more cathode propellent with the EOP, this should be evident in an increase of discharge current in the EOP plots compared to the OOP plots of Figure 8.3. If the EOP configuration was 
ingesting more propellent, we would expect higher discharge currents at $r=70 \mathrm{~mm}$, and, in fact, for every point $70 \mathrm{~mm} \leq r \leq 100 \mathrm{~mm}$ where the cathode was in front of the face of the EOP but not the OOP. Instead, very similar discharge currents are seen across this range. Finally, we can "correct" the anode efficiency by assuming that in the case of the EOP all cathode neutrals were ingested and singly-ionized. To perform this correction, we simply take the original equation for anode efficiency (Equation 2.22) and increase the mass flow in the denominator appropriately

$$
\eta_{a, \text { corrected }}=\frac{T^{2}}{2 \dot{m}_{a} I_{d} V_{d}} \frac{\dot{m}_{a}}{\dot{m}_{a}+\dot{m}_{c}}
$$

which, of course, yields the same thing as the total efficiency, Equation 2.23. In Figure 9.1 we see that the total efficiency of the EOP thruster is higher than the anode efficiency of the OOP, and therefore cathode propellent ingestion cannot be the source of the improvement.*

A final argument can be made that the thruster was not operating with its nominal propellent (xenon) and therefore these results should not be generalized, particularly given that our thruster ran unexpectedly poorly on krypton (see Section 8.1). Given the magnitude of the change in efficiency and cathode coupling voltages seen here, however, it seems unlikely that changing to nominal conditions would nullify these results. None-the-less, we would not necessarily expect to see the same 10-point improvement for a well tuned, OOP-type thruster operating at around 50\% efficiency. The magnitude of the improvement in this case is presently unknown and is a topic for future research.

\footnotetext{
*As an aside, it is worth pointing out that cathode propellent ingestion is probably good for the total efficiency of the thruster, assuming that the ingestion does not negatively affect any of the ionization processes occurring in the thruster. As long as the cathode propellent must be expelled, better to have it be turned into thrust-generating fast ions than to have it hang around the thruster as slow neutrals, or worse, back-flowing charge-exchange ions.
} 


\section{Chapter 10}

\section{Conclusion}

\subsection{How Cathode Position Affects Efficiency}

The goal of this research was to understand how cathode position affects cathode coupling and thruster performance. Based on the data presented, several conclusions can be drawn. First, several oft-observed phenomena have been confirmed. Cathode position does affect thruster performance, cathode coupling, and near-field plume potential, as can be seen in Figures 7.2, 8.3 and 8.17. Furthermore, an increased cathode mass flow rate improves the cathode coupling voltage and the anode efficiency.*

The relationship between cathode mass flow rate and thruster performance seen here and in many other studies (see Chapter 3) provides a compelling, if incomplete, argument that the external magnetic field plays an important role in the cathode coupling. The increased neutral density that results from increased $\dot{m}_{c}$ provides for increased cross-B-field electron mobility via electron-neutral collisions. Simultaneously, one also expects increased creation of free electrons from the cathode as more target neutrals are introduced through the cathode.

However, the way in which cathode position affects performance is not simple. The data presented in this work show a consistent trend. The thruster has a minimum in performance with the cathode placed radially just outside of the outer pole. As the separation between the cathode and the thrust-centerline is increased from this position, efficiency improves slightly, while as the distance is decreased from this position, the efficiency improves dramatically, at least up to where the cathode begins to physically block the exit channel. Figure 8.3 provides good examples of this. Furthermore, as the cathode is moved down stream, the efficiency improves significantly, as can be seen

${ }^{*}$ The effect of cathode mass flow on total efficiency is less clear in this work, with the thruster showing, on average, slightly improved total efficiency with increased $\dot{m}_{c}$ for the OOP in the separatrix crossing experiment, while showing slightly decreased total efficiency with increased $\dot{m}_{c}$ for the EOP. Again, see Figure 8.3. 
in Figure 7.2. In the only other study to perform measurements across a sufficient range to make comparisons, a dissimilar behavior was noted. ${ }^{1}$ Of course, there are several differences between the two experiments. Most noteably, Walker and Gallimore performed measurements of a much larger displacement range with lower resolution and studied a different thruster than the BPT-2000 used here.

The data presented in Figure 8.12 clearly show that cathode position affects all components of efficiency. The correlation between beam divergence, cathode coupling voltage, and plume voltage shown in Sections 8.6 and 8.7 presents a compelling case for one mechanism whereby cathode position affects thruster performance. As the cathode is moved to less favorable locations (e.g. at $r=100 \mathrm{~mm}, z=30 \mathrm{~mm}$ on this thruster) the system compensates for the higher electron impedance by increasing the potential in the near-field and pushing the cathode down lower. This has the net effect of increasing the potential difference that drives the electrons from the cathode to the beam. However, the increased potential in the near-field of the plasma increases the divergence of the beam. Therefore, the thruster experiences a two-fold decrease in efficiency due to the mechanism. First, less energy is available to the ions for acceleration, that is, $\eta_{\mathrm{Vcg}}$ decreases. The second, stronger effect is that the accelerated ions are not as focused, that is, $\eta_{\theta}$ decreases.

As was discussed in Section 8.5, the voltage utilization and velocity distribution efficiencies actually decrease with decreasing radial cathode position, partially counteracting the gains in divergence and cathode coupling efficiency. As was suggested before, the decrease in $\eta_{V}$ and $\eta_{\mathrm{vdf}}$ may be due, in part, to cathode propellent ingestion. It was further explained that cathode propellent ingestion was insufficient to explain the increase in current utilization seen. Combining the plume data into the analysis allows for another possibility. As can been seen in the plume data of Section 8.6, there are some cathode positions where the near-field plasma potential is higher and, therefore more "drawn out" from inside the discharge chamber as shown in the sketch of $V_{p}$ vs. $z$ in Figure 10.1. To state this more technically, the average gradient of $V_{p}$ from the anode to the exit plane is smaller. If the ionization region moves only slightly while the plasma potential is drawn out (which seems likely given that the ionization region is typically near where the magnetic field is strongest), then the region of strongest ionization may no longer overlap a region of steep plasma potential. The result of this is that the ions created in the ionization region will drift slowly, rather than being immediately expelled from the thruster. This provides a higher chance that they will recombine (primarily through wall collisions) before finding their way to the steeper potential gradient and, thus, exit the thruster as neutrals. This will result in a lower beam current and, therefore, a lower $\eta_{I}$. However, those ions that are expelled will have, on average, higher energy, that is, $\eta_{V}$ will be greater. Furthermore, because the high potential region covers a larger portion of the ionization region, the overall spread of ion energies 
will be decreased as IEDF becomes more sharply peaked at high energies, that is $\eta_{\mathrm{vdf}}$ will be greater.

\subsection{How Cathode Position Affects Cathode Coupling}

The changes in near-field plasma potential can be directly linked to most of the changes in efficiency of the thruster. However, this leads to the question, "How does changing cathode position cause a change in near-field plasma potential?" As was shown in Chapter 6, the plasma in the region of the HET investigated here is magnetized, with Hall parameters greater than 20 and Larmor radii less than $5 \mathrm{~mm}$ (see Figure 6.8). Therefore, we expect electron transport across the magnetic field lines to be strongly impeded, requiring collisions with neutrals, or some other similar event* to enable the electrons cross the field lines. Increasing the distance between the cathode and the beam plasma creates a greater magnetic barrier for the electrons originating at the cathode. For electrons to have sufficient energy to overcome this increased barrier (thereby sustaining the plasma) the potential in the near-field plume must be increased, the potential of the cathode decreased, or both.

Further evidence of this interpretation can be found in the creation of the doublelayer, as discussed in Section 8.6. For illustrative purposes, we assume that the only mechanism by which the electrons cross the magnetic field is through collisions with neutrals. Combining the magnetic field data from Section 6.3, the Hall parameter data from Section 6.5.2 with the experimental $T_{e}, n_{e}$, and electric field data derived from $-\nabla V_{p}$, we can create a view of the classically induced electron current densities within the plasma according to Equations 2.19 and 2.18. Figures 10.2 and 10.3 show the results of this analysis for the $\dot{m}_{c}=10$ SCCM krypton experiments with the original outer pole. The magnitude sub-figures show the $\log$ (base 10) of the magnitude of the electron flux in electrons $/ \mathrm{s} \mathrm{m}^{2}$, while the angle sub-figure shows the angle relative to the thrust axis at which this flux flows. This angle can also be seen in the arrow plots overlaying the color maps. Note that the arrows do not provide magnitude information.

Figure 10.2 shows the presence of the double layer and its effect. This effect can be seen in all of the OOP data ${ }^{\dagger}$ where the cathode position is greater than $60 \mathrm{~mm} .^{\ddagger}$ When the cathode is positioned outside the separatrix, as is shown in Figure 10.2, a

\footnotetext{
*Plasma fluctuations are often considered "collisional" events if they provide the necessary kick to move an electron from one field line to the next.

${ }^{\dagger}$ The EOP may also show the same phenomenon, however, it presents less clear of a picture. This is likely due to the decreased magnetic field strength necessary to run the EOP plate, or due to the increased instability of operation of the EOP resulting in poorer quality probe data.

$¥$ Admittedly, the $120 \mathrm{~mm}$ case was chosen because it shows the clearest example of this phenomenon.
} 
Lower average near-field potential

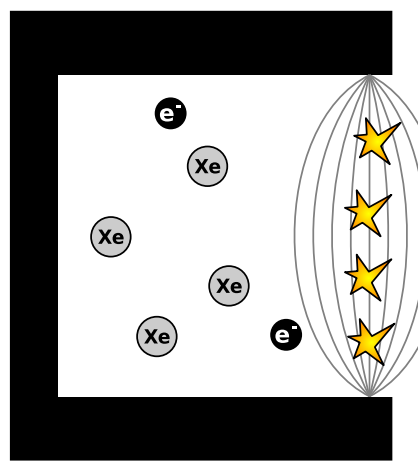

Well collimated

beam

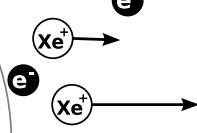

- $\longrightarrow$

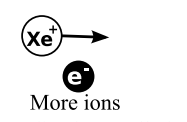

immediately expelled
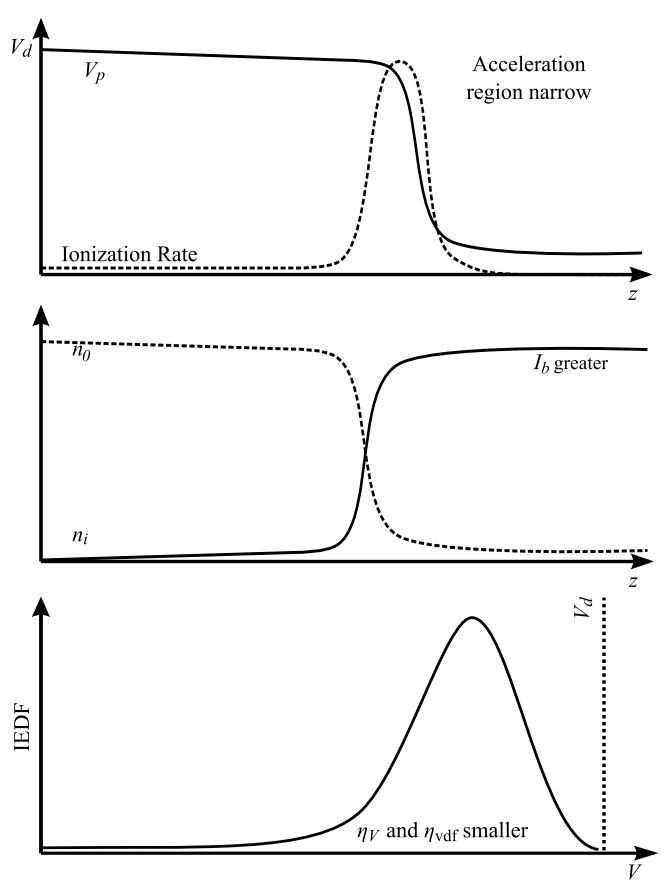

Higher average near-field potential
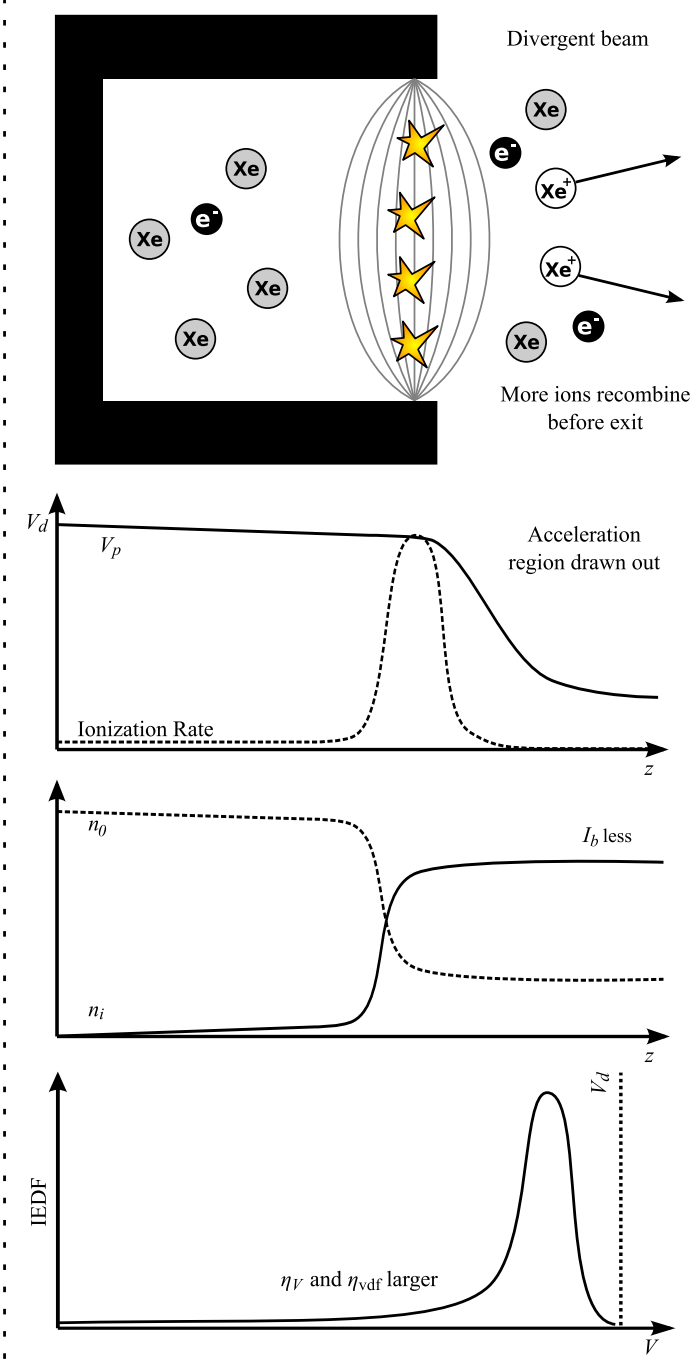

Figure 10.1: Effect of drawn out plasma potential on efficiency components 

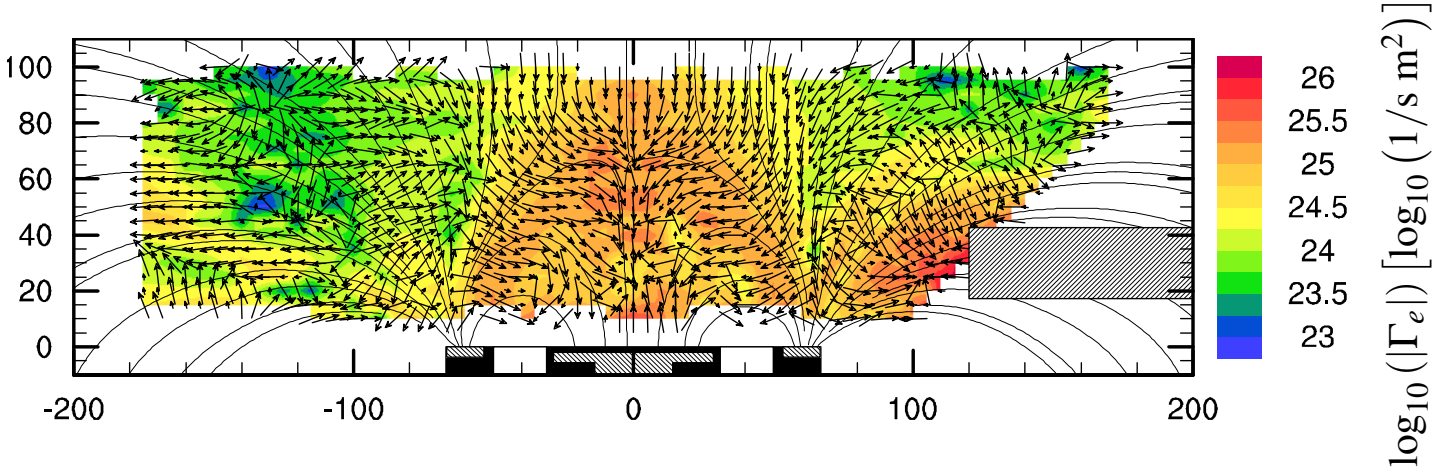

(a) Magnitude

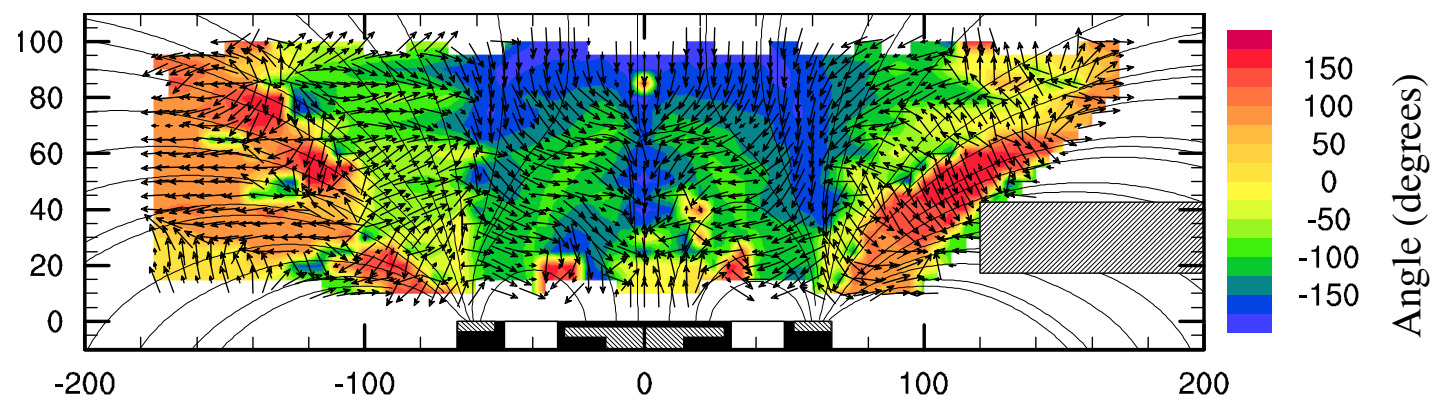

(b) Angle

Figure 10.2: Electron flux densities assuming classical mobility for the OOP with $\dot{m}_{c}=10 \mathrm{SCCM}$ and $r=120 \mathrm{~mm}$

boundary forms along a magnetic field line. In this case the boundary is clearly seen by the mobility direction plot, Figure $10.2 \mathrm{~b}$. The red areas outside the cathode actually indicate electron current toward the cathode, while on the other side of the boundary the current is toward the beam, as is expected.

For this analysis to make sense, we must realize that the potential map is not complete. There must be a sharp drop to a region of lower potential near the cathode. In fact, investigating the potential map in Figure C.15 we realize that this must be the case, as the lowest plasma potential mapped is $0 \mathrm{~V}$ (relative to ground) and the cathode is known to roughly $32 \mathrm{~V}$ below ground. A second caveat is that the electron mobility model used here assumes an isotropic, Maxwellian electron energy distribution. If the electrons enter any region with directed energy, then these figures cannot represent the actual electron flux. None-the-less, the figures give an approximate indication of the forces acting on electrons in the plasma.

With this in mind, it is obvious that for an electron to be transported from the cathode to the ion beam it must first pass over the double-layer. (The double-layer is seen most clearly in the the red-green boundary near the cathode in Figure 10.2b.) How- 

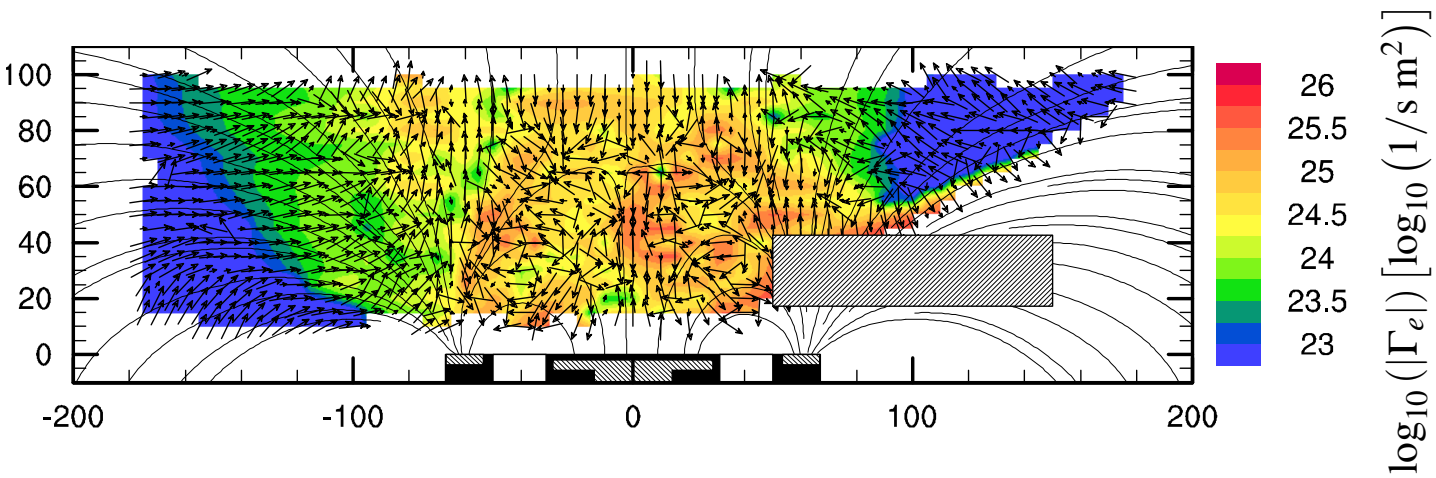

(a) Magnitude

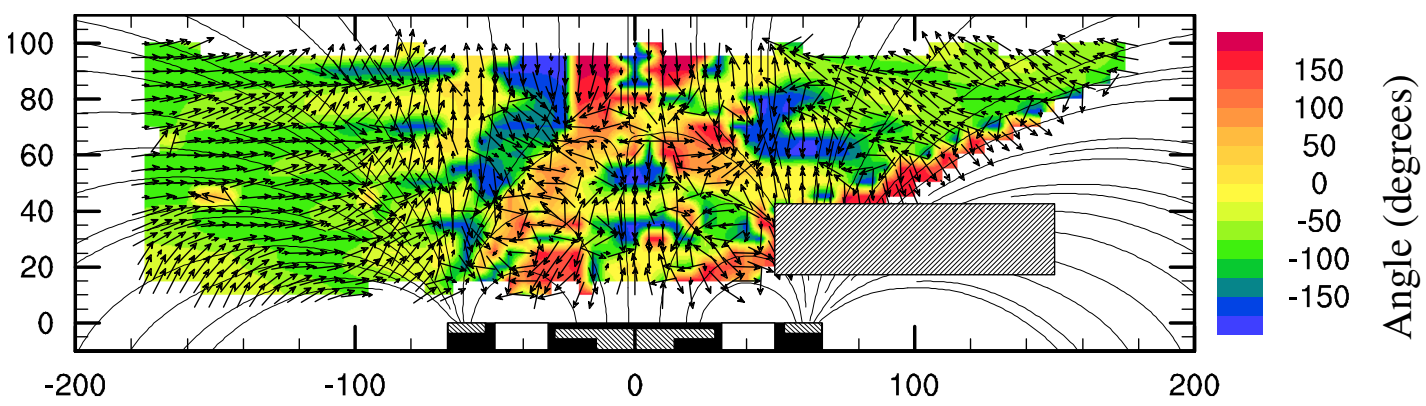

(b) Angle

Figure 10.3: Electron flux densities assuming classical mobility for the OOP with $\dot{m}_{c}=10$ SCCM and $r=50 \mathrm{~mm}$

ever, in cases where the cathode is already inside the separatrix, the double layer never forms, as is exemplified by Figure 10.3. When the cathode is positioned inside the separatrix, the magnetic field lines form a pathway which carry the electrons into the beam, assisting rather than impeding the coupling.

\subsection{The Separatrix}

The preceding arguments show that the magnetic field can either impede or assist the cathode coupling depending on the location of the cathode within the magnetic field topology. By placing the cathode inside of the separatrix, the field is guaranteed to assist the coupling. As discussed in Section 6.7, some thrusters may not exhibit a separatrix or may exhibit it in a location such that it does not significantly affect that cathode coupling. However, for those designs which exhibit a separatrix near the ion beam, Chapter 8 presents a modified thruster design which allows for easier coupling while still keeping the cathode out of the damaging ion beam. Chapter 9 shows that by 
changing only the contour of the external magnetic field so that the separatrix is moved outside of the cathode location, the performance can be improved.

This conclusion also explains the improved performance associated with internally mounted cathodes. ${ }^{2,3}$ The internally mounted cathode is naturally inside the separatrix. Furthermore, the data from these studies report lower coupling voltage magnitude, lower near-field plume plasma potential and lower beam divergence with the internally mounted cathode. These data are consistent with the results of this work.

Perhaps most importantly, the establishment of the importance of the separatrix, as shown in Chapter 8, and, in particular, Section 8.6.3, suggests that the consideration of the separatrix should become a standard part of cathode placement criteria. Beyond this, future Hall thruster designers should give consideration not only to the internal field generated by their magnetic circuit, but also to the feasibility of placing the cathode within the separatrix generated by their magnetic circuit.

\subsection{Future Work}

This dissertation adds to the understanding of cathode coupling processes in Halleffect thrusters and of the importance of the external magnetic field. However, there are still several aspects of this work that need further verification or more research. First and foremost, these experiments were conducted with one thruster, one cathode, and only two, very similar magnetic field topologies. While I expect that the work can be generalized to any Hall thruster, further work is necessary for confirmation.

Second, most of this research was performed using the non-optimal propellent krypton. Furthermore, the performance on krypton was worse than expected for reasons which are not understood. While the efficiency improvements seen when switching from the original outer pole to the extended outer pole are still expected with xenon, the magnitude of the improvement is unknown. Also, in most of the experiments the magnetic field was held constant in order to maintain a constant field across each trial. It was important to do this in order to minimize the number of variable parameters in these experiments. However, it also means that the thruster may have been at nonoptimal magnetic fields for many of the experiments. The experiment in Chapter 9 mitigated this problem, but no probe data were taken. Therefore, a similar study to that of Chapter 8 using xenon as a propellent and with the magnetic field adjusted to the optimal value at each position would be beneficial.

Third, the effect of the cathode neutral flow field, and, in particular, cathode angle has not been studied in detail in this work. Given the strong correlation between neutral flow rates and cathode coupling voltage, along with the importance of magnetic fields, a more detailed study of the effect of the cathode plume is warranted.

Finally, the design of the extended outer pole in these experiments was crude. A careful design of the EOP needs to carefully consider the effect of the pole on both the 
inner and the outer field topologies. Furthermore, there may be alternate techniques of achieving the same goal, namely, of having the cathode inside the separatrix but outside the ion beam. Certainly the oft-studied center-mounted cathode is one such technique applicable to large HETs. For smaller HETs, other magnetic field topologies may be created that would perform the same function. In addition to modified outer poles, there are other ways of modifying the external magnetic field to improve the cathode coupling. For instance, it may also be beneficial to add auxiliary magnetic coils to the cathode in order to modify the local field. Creative engineering applied to this problem will no doubt find more elegant solutions than the EOP used here.

\subsection{Epilogue}

Hall-effect thrusters are one of the simpler classes of electric propulsion devices. They have been under development for over 60 years. Even so, there are still several aspects of the device that are still not well understood. Cathode coupling, which is effectively the study of the interaction of two plasmas in the presence of a magnetic field, is one of these areas. It is my hope that the data and conclusions provided in this work will be of use to future researchers and developers. It is likely that improvements such as a well-designed EOP will lead to only modest gains in efficiency. However, even modest gains may make previously impossible missions possible. More importantly, it is only through the understanding that comes from the study of the basic phenomena such as this coupling process that breakthrough improvements can be made.

\section{References for Chapter 10}

[1] Walker, M. L. R. and Gallimore, A. D., "Hall Thruster Cluster Operation with a Shared Cathode," Journal of Propulsion and Power, Vol. 23, No. 3, May 2007, pp. 528-536.

[2] Hofer, R. R., Johnson, L. K., Goebel, D. M., and Fitzgerald, D. J., "Effects of an Internally-Mounted Cathode on Hall Thruster Plume Properties," 42nd AIAA/ASME/SAE/ASEE Joint Propulsion Conference, July 9-12, 2006, Paper No. AIAA2006-4482.

[3] Jameson, K. K., Goebel, D. M., Hofer, R. R., and Watkins, R. M., "Cathode Coupling in Hall Thrusters," 30th International Electric Propulsion Conference, Florence, Italy, September 17-20, 2007, Paper No. IEPC-2007-278. 


\section{Appendix A}

\section{Code Listings}

This appendix shows the code listings for select algorithms used in this dissertation. These algorithms are implemented in either $\mathrm{C}$ or Python. Those algorithms implemented in Python typically require the NumPy and SciPy packages, as noted by the import statements at the top of these files. The code comments conforms to Doxygen syntax, and HTML documentation can be generated from them.

\section{A.1 Langmuir Probe Processing Algorithm}

This section contains the code listing for the double Langmuir probe processing algorithm as described in Section 5.4. The Langmuir probe processing algorithm is divided into two files. The first, peterson.py, provides the forward algorithm, utilizing the analysis of Peterson and Talbot. ${ }^{1}$ Given the plasma parameters such as $T_{e}$ and $n_{e}$, and probe dimensions, it calculates the expected probe trace. The main implementation of this for double probes is in the PetersonTalbotDouble class. The second file, langmuir.py, performs the reverse algorithm. Given experimental probe traces and probe dimensions, it finds the plasma parameters. Details are given in the DoubleGndProbe class.

\section{A.1.1 peterson.py}

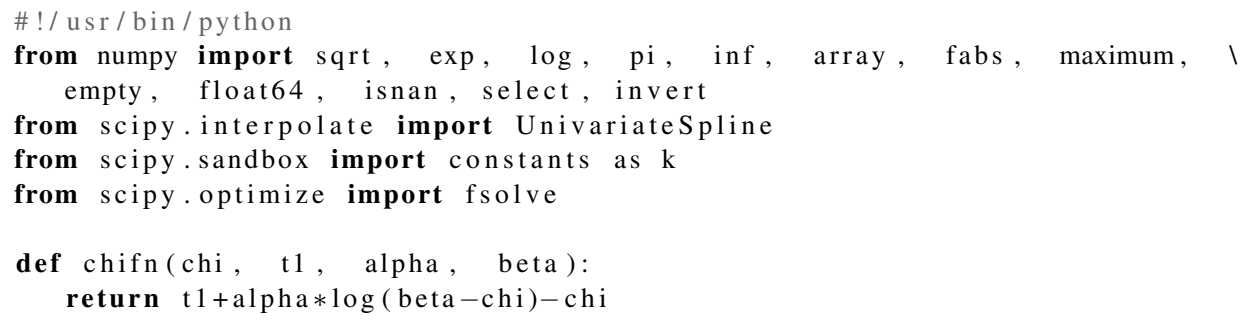




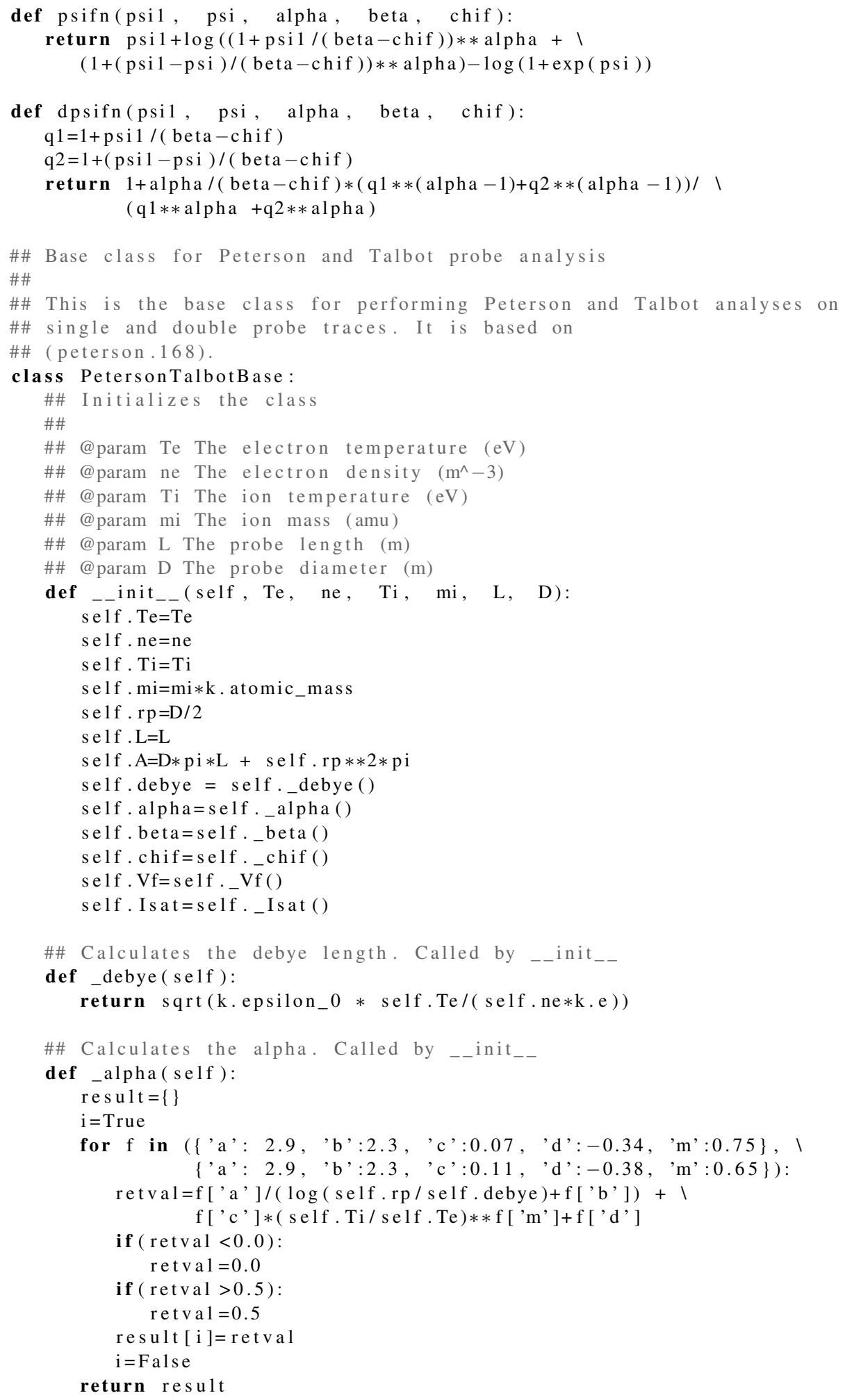




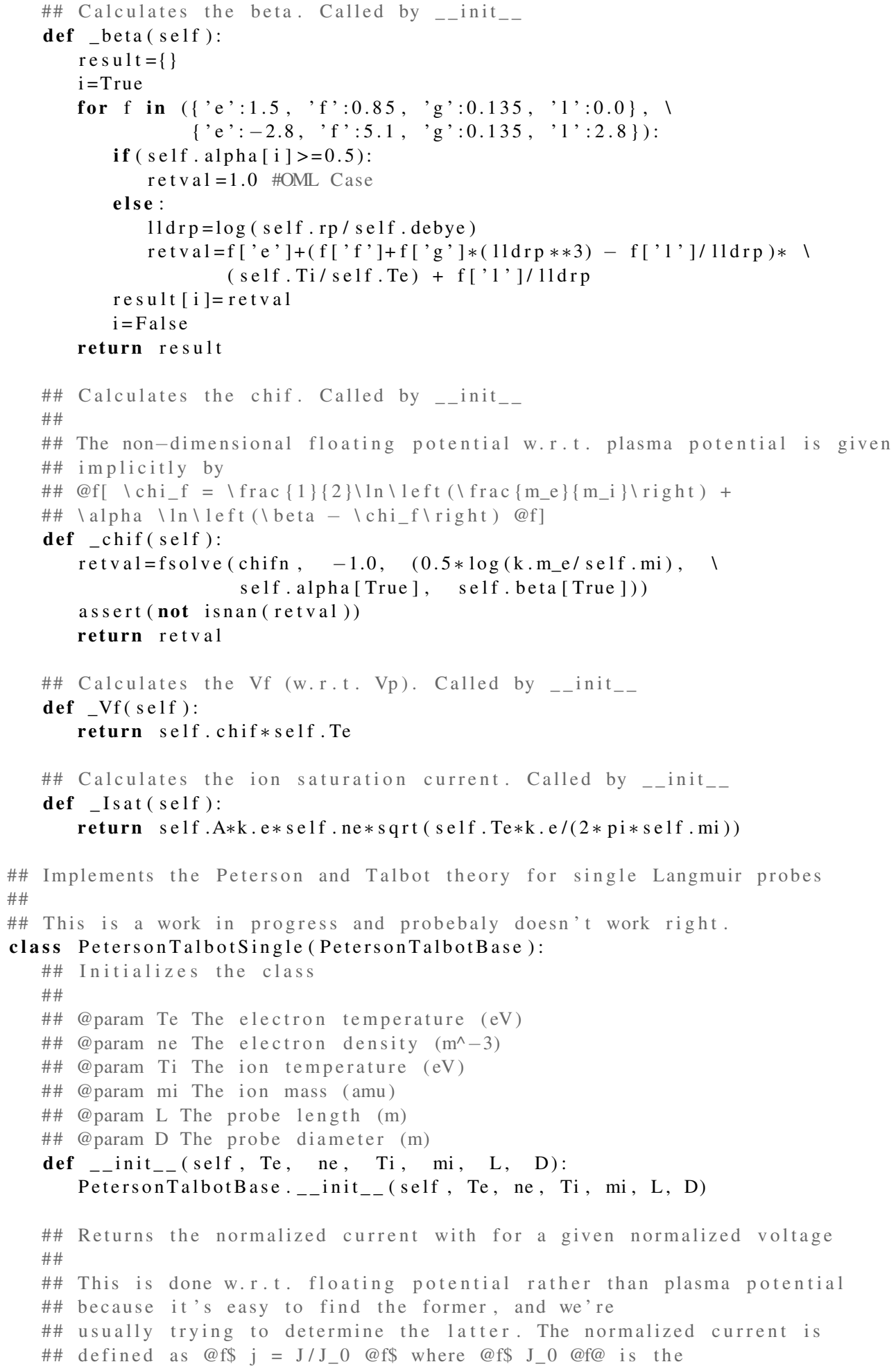




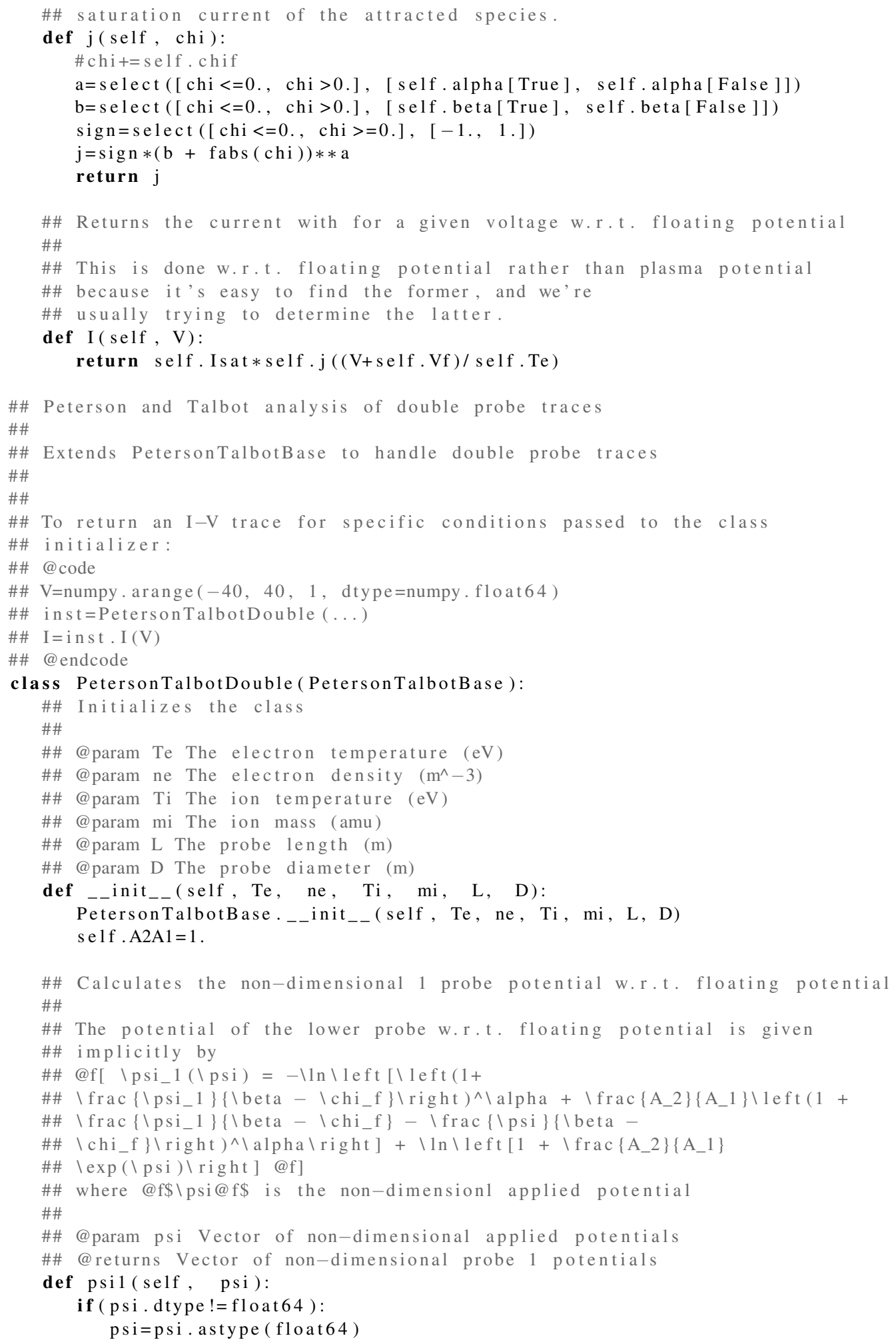




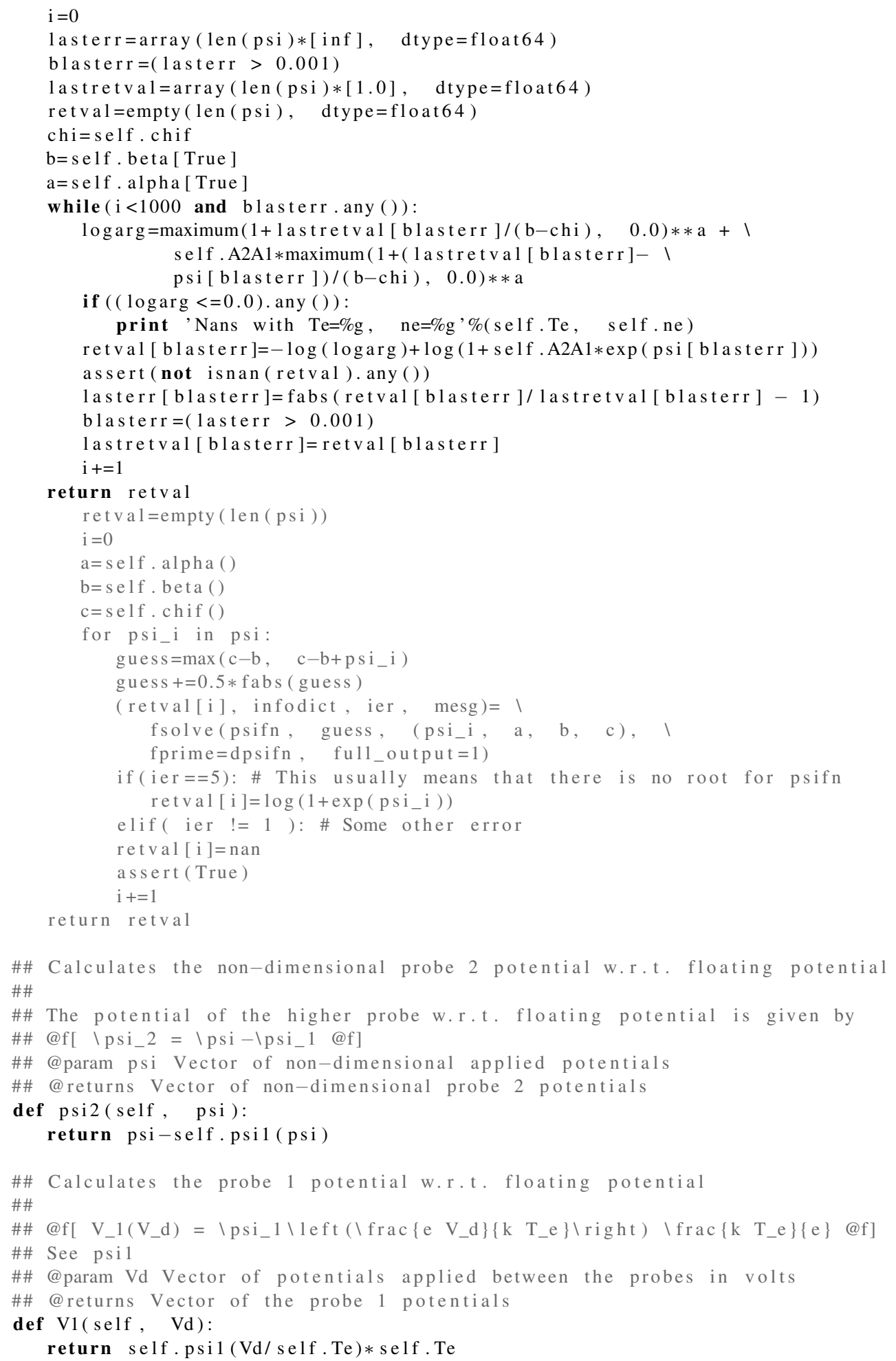




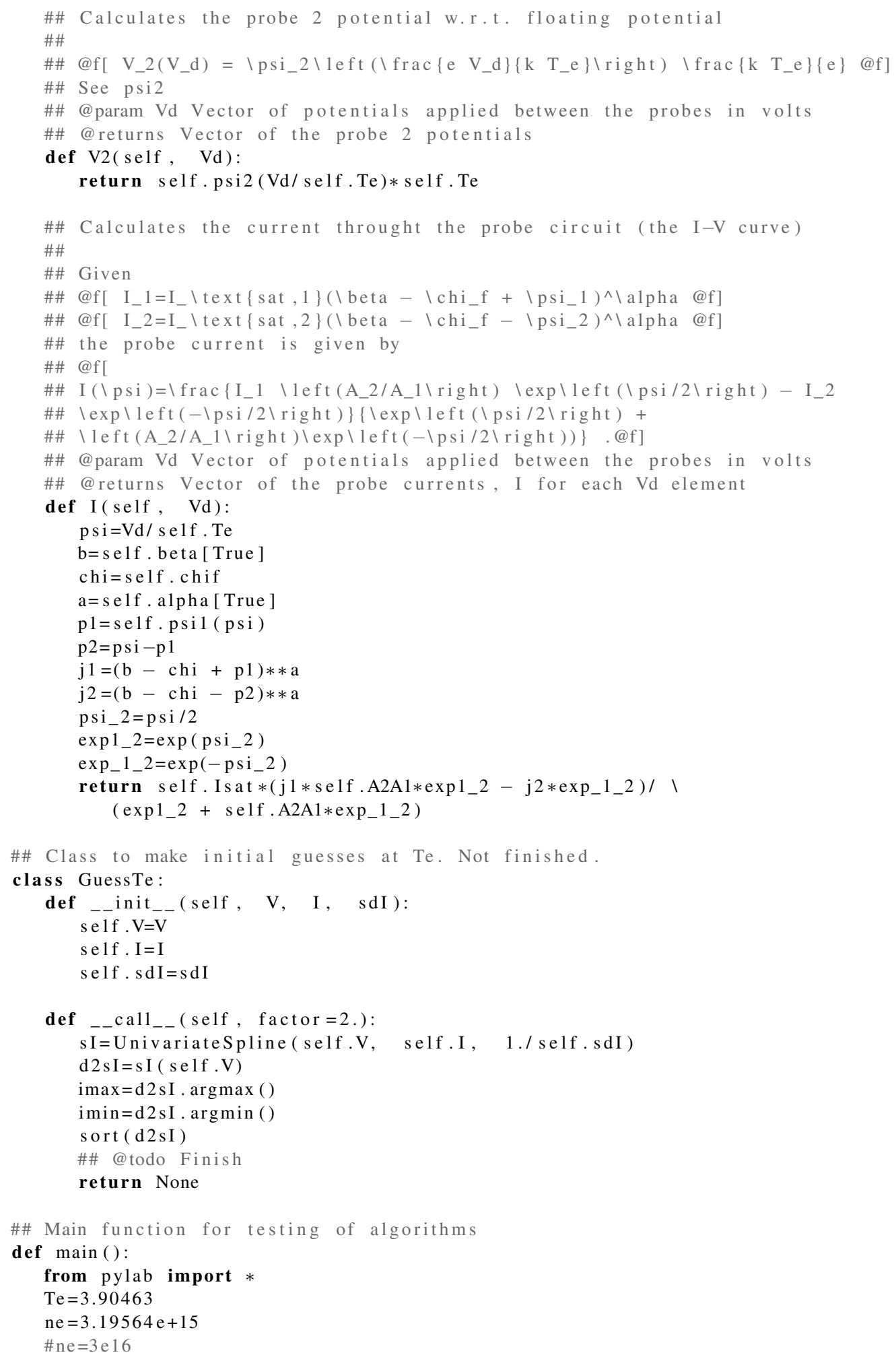




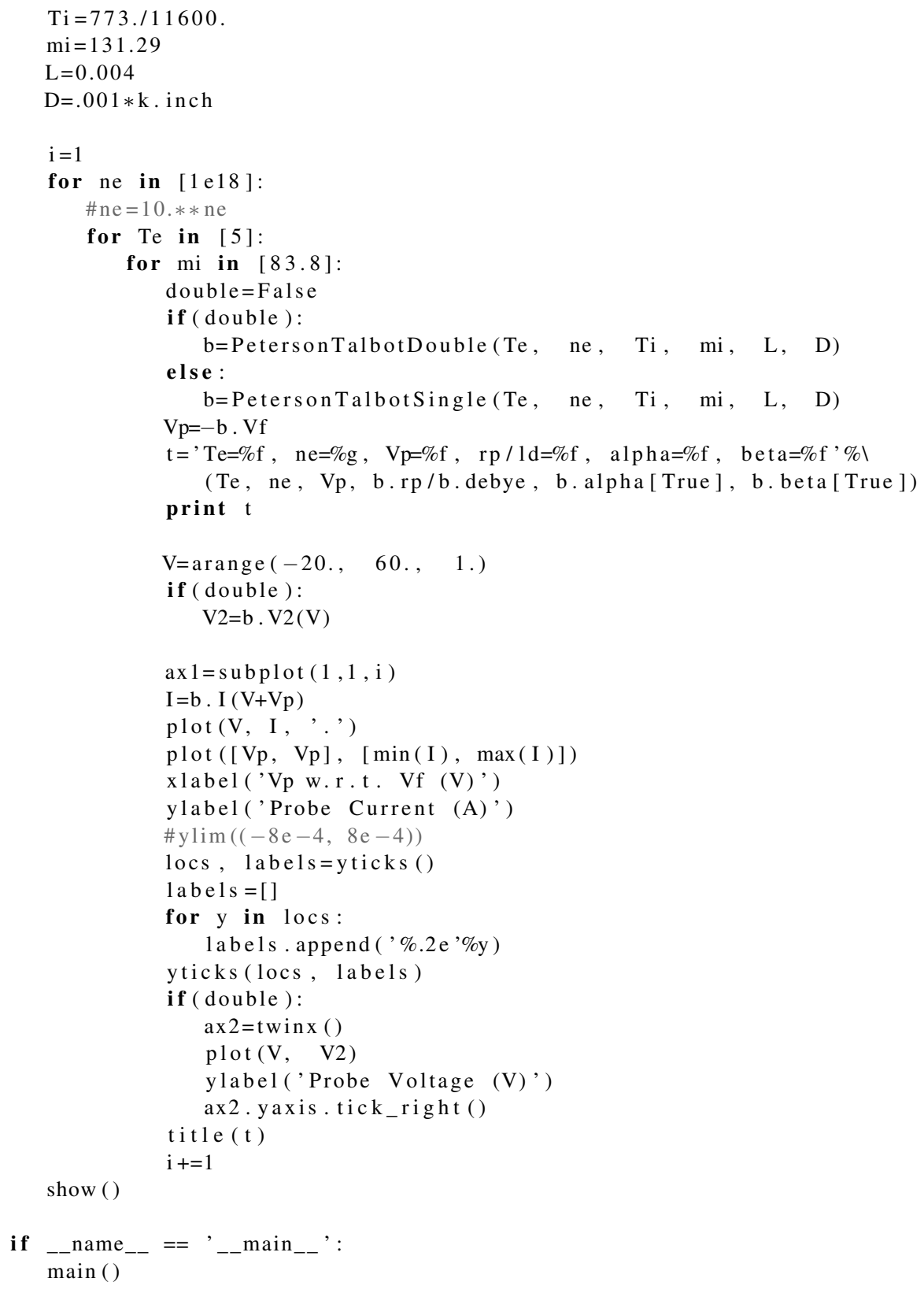

\section{A.1.2 langmuir.py}

\#!/ usr/bin/python

import sys

sys.path . append ('/home/jdsommer/Research/cathode/exp1/Software/')

from scipy. interpolate import interp $1 \mathrm{~d}$

from scipy.optimize import *

import scipy. sandbox. constants as k

from scipy import polyfit 


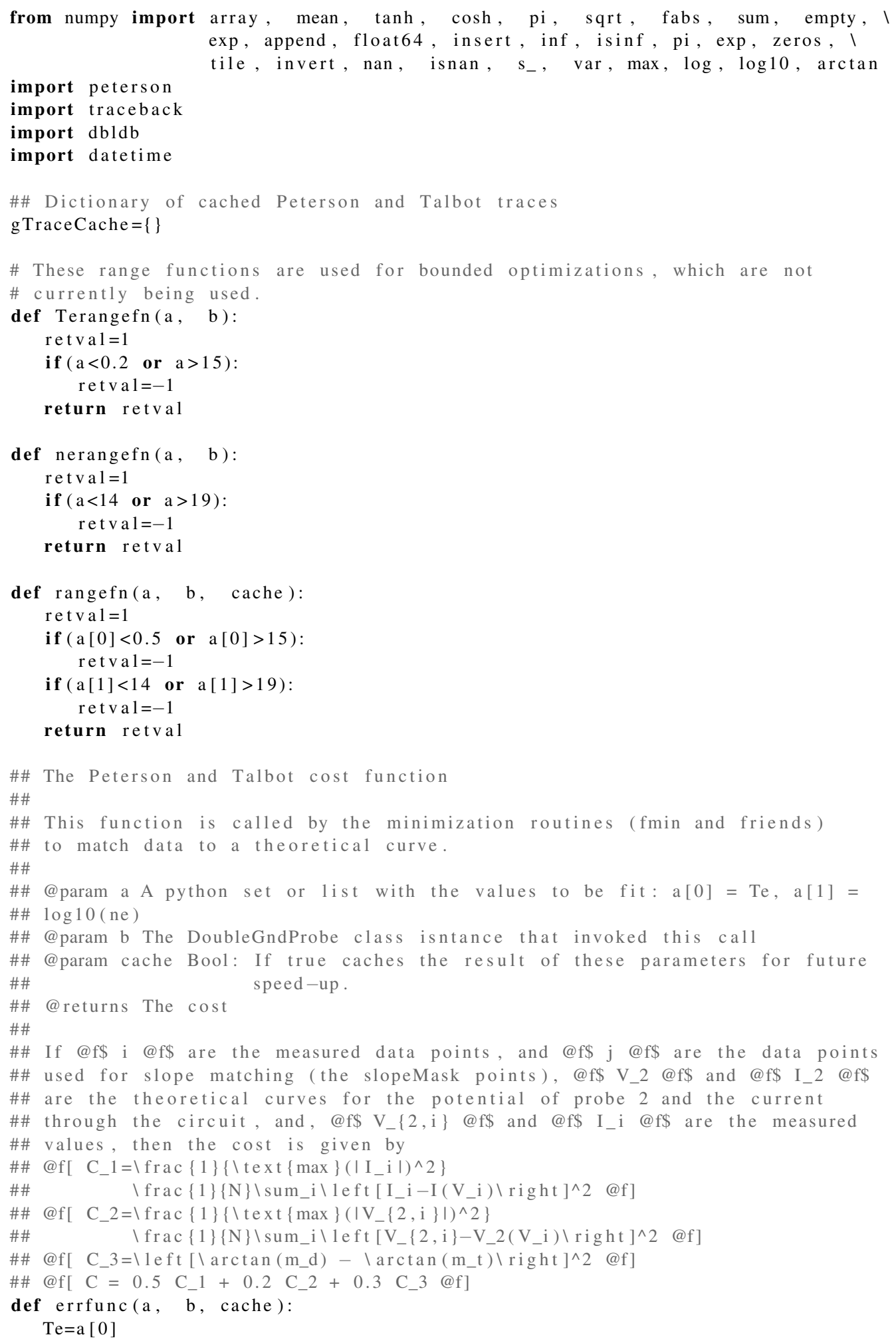




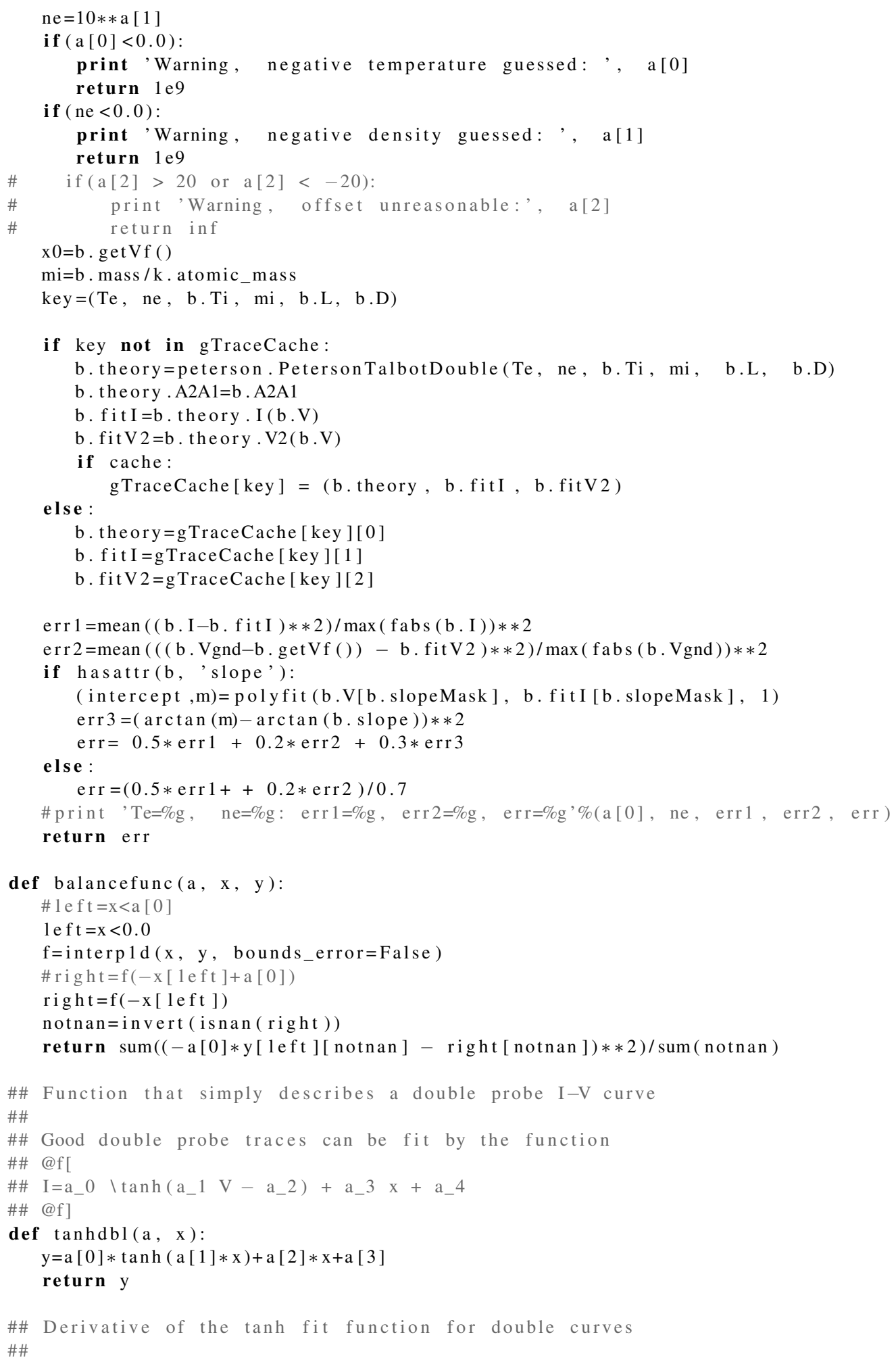




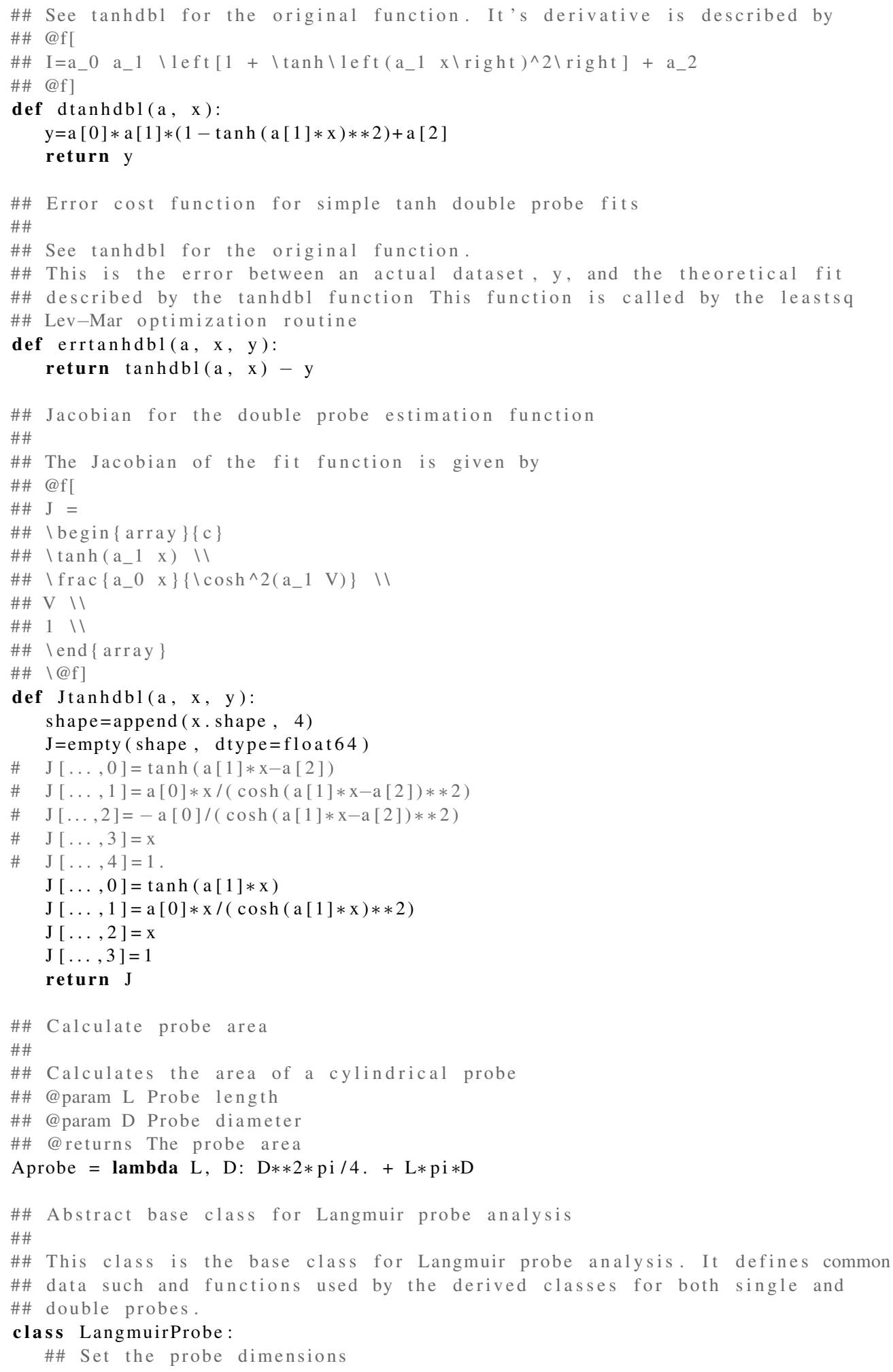




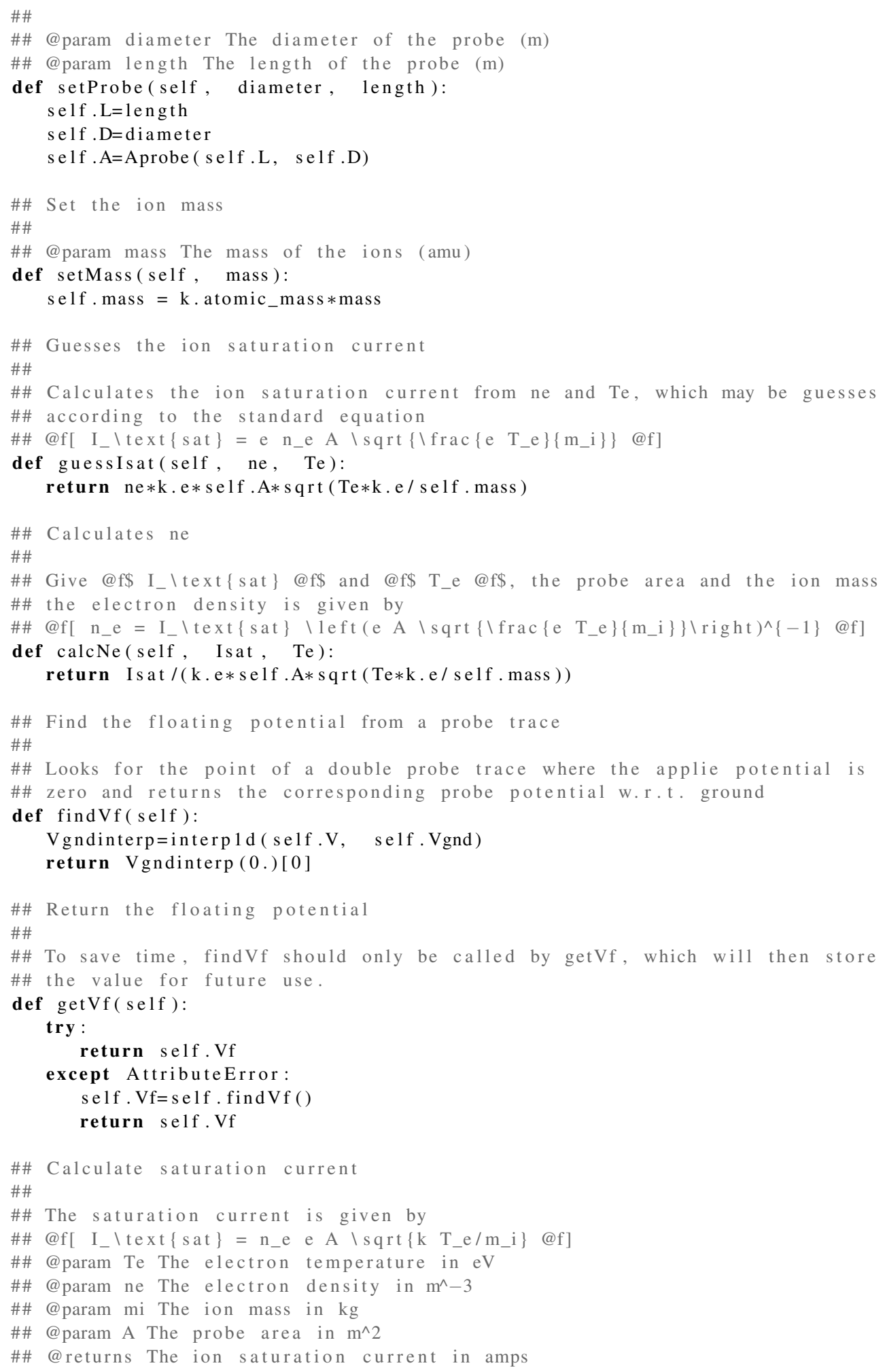




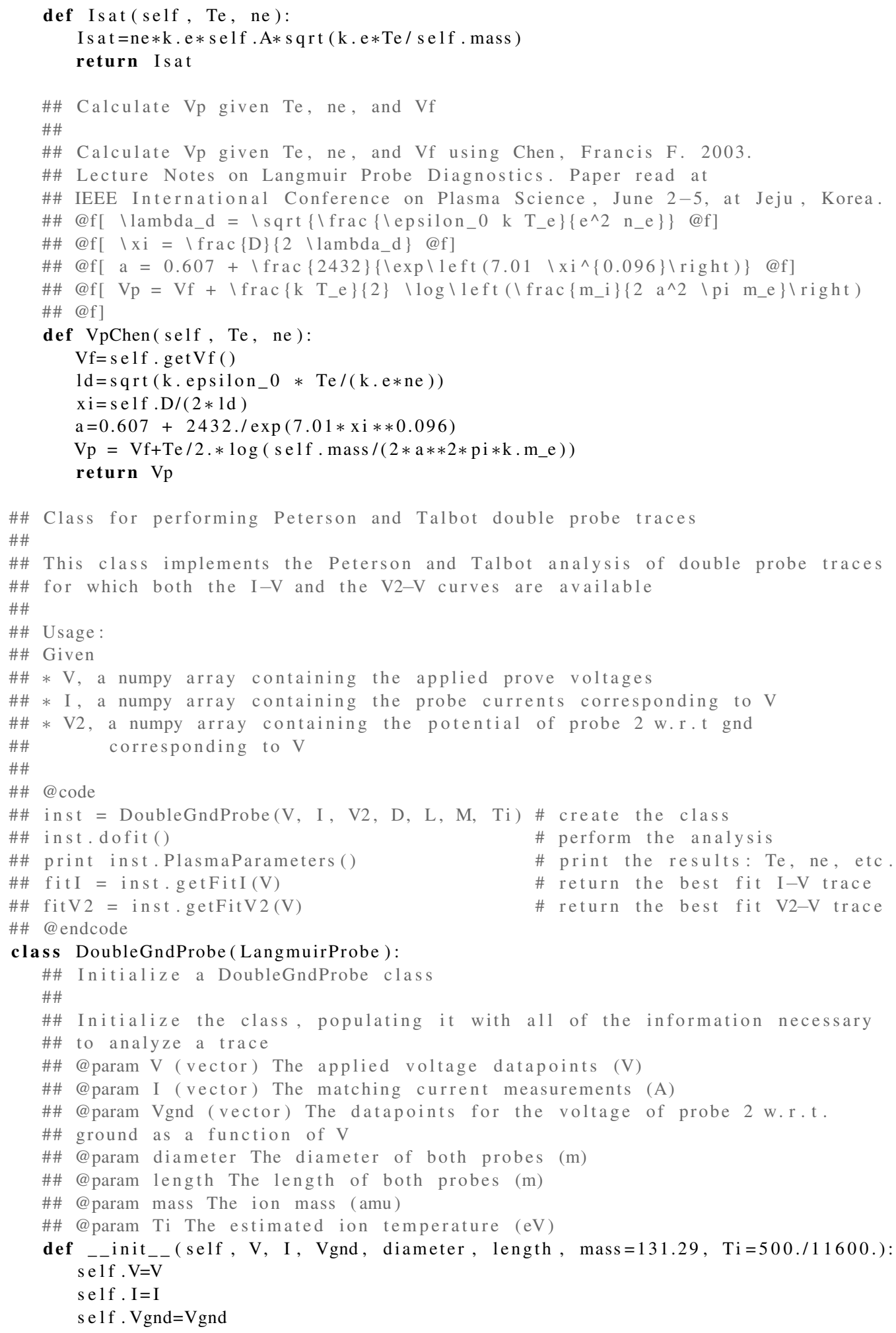




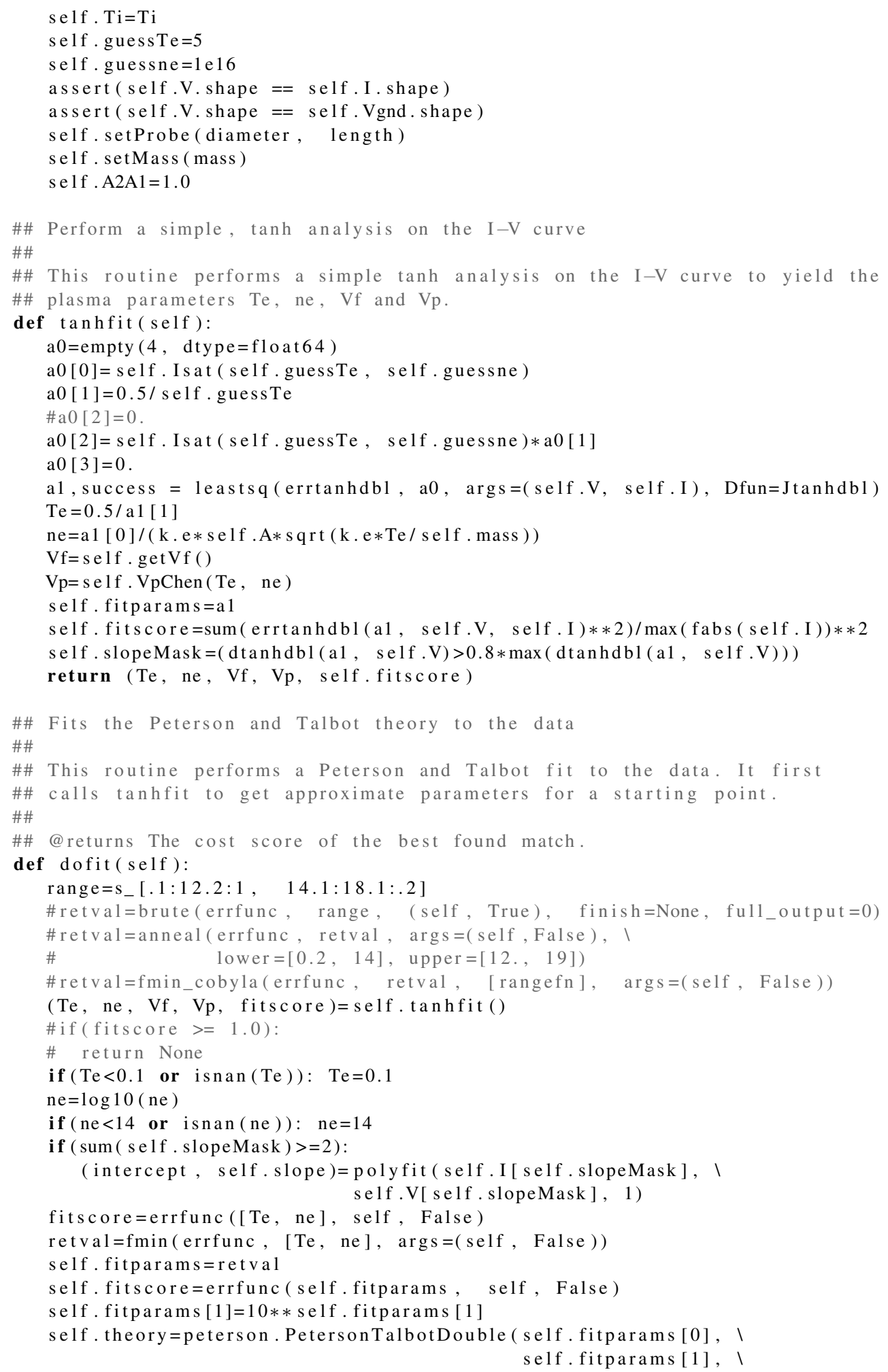




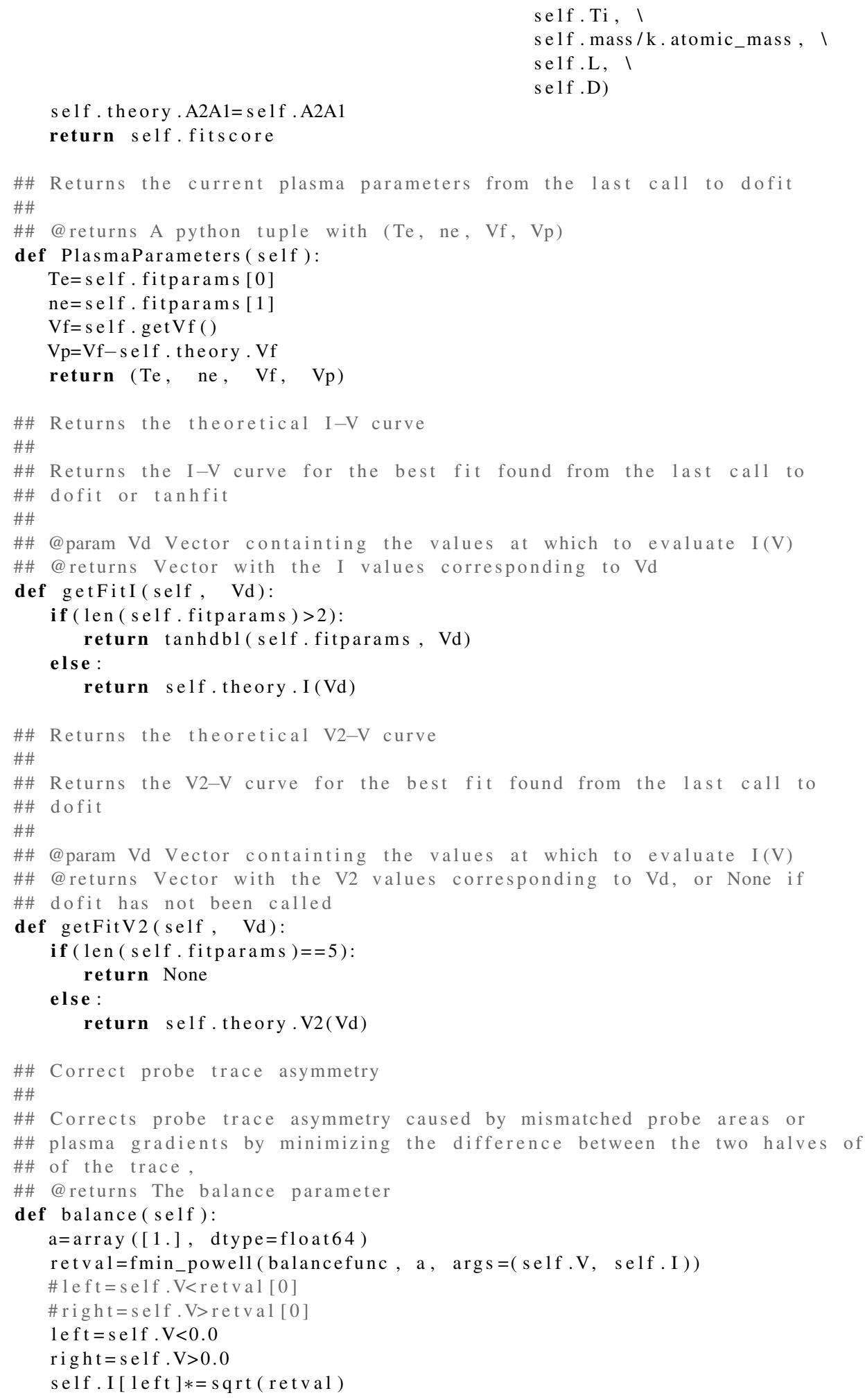




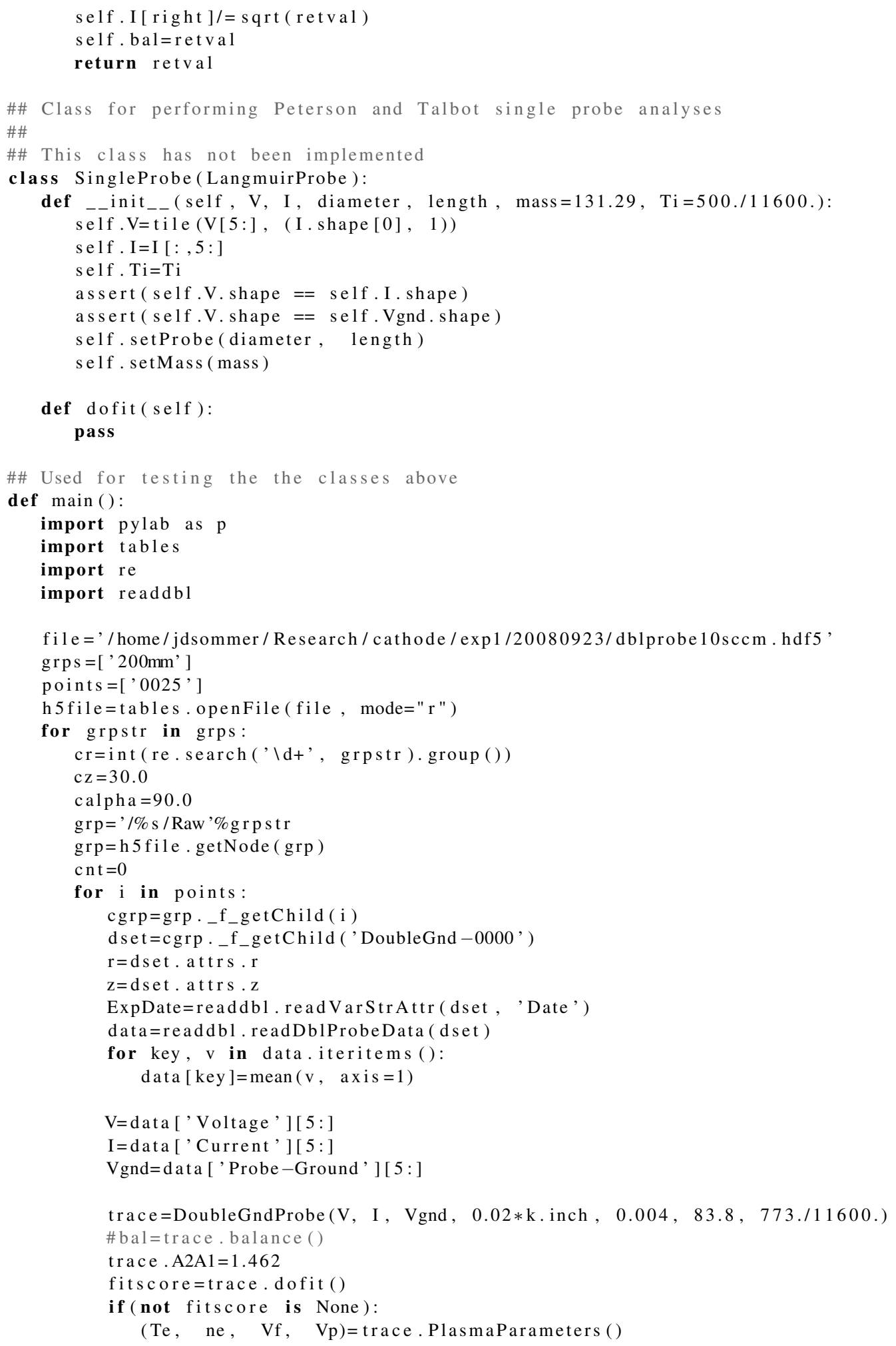




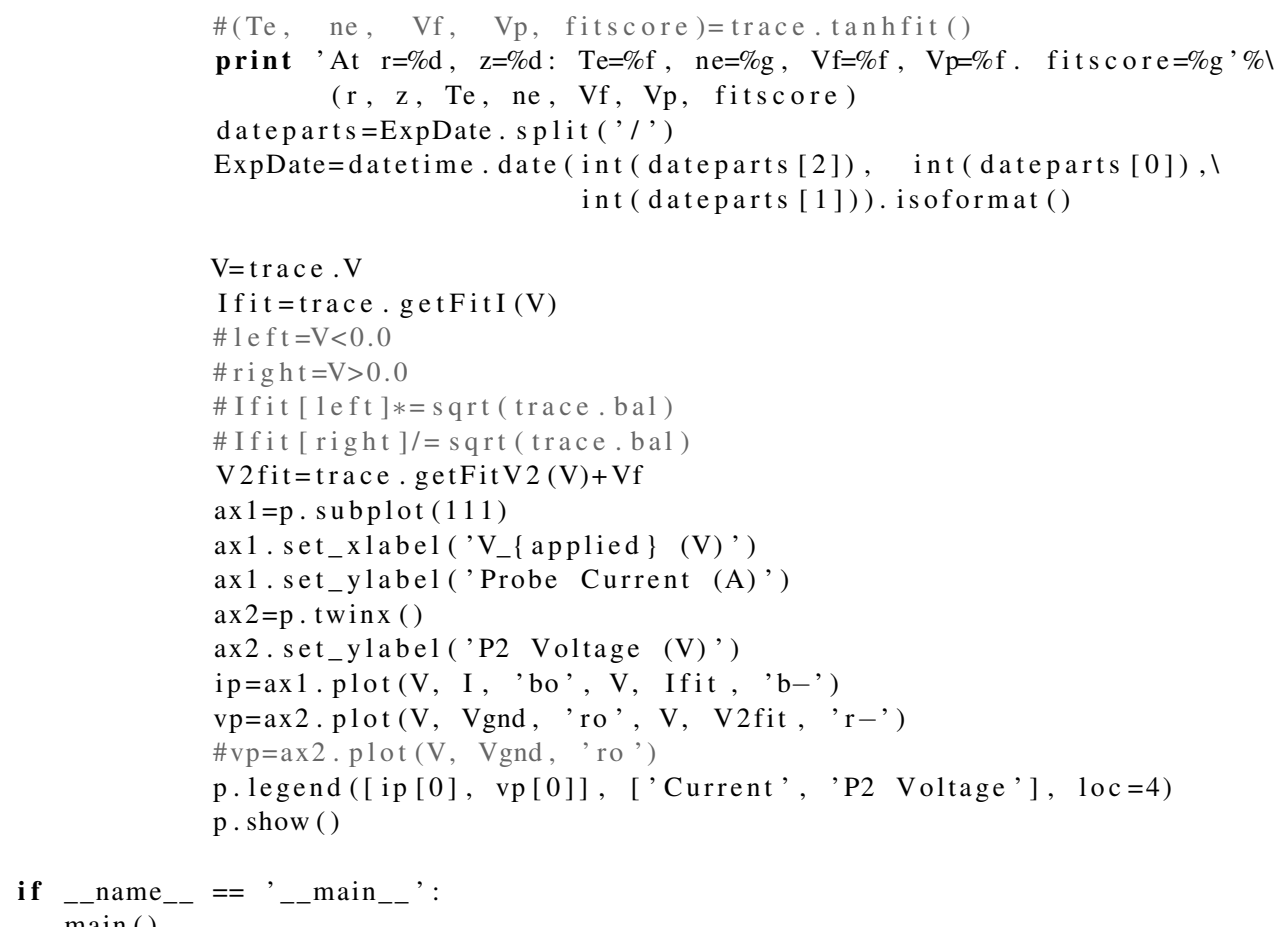

\section{A.2 Savitzky-Golay Smoothing Algorithm}

The Savitzky-Golay algorithm ${ }^{2}$ is to smooth RPA traces in this work (see Section 5.3). This implementation is based on the work by Gorry. ${ }^{3}$ The main entry point for the algorithm is the function sgsmooth.

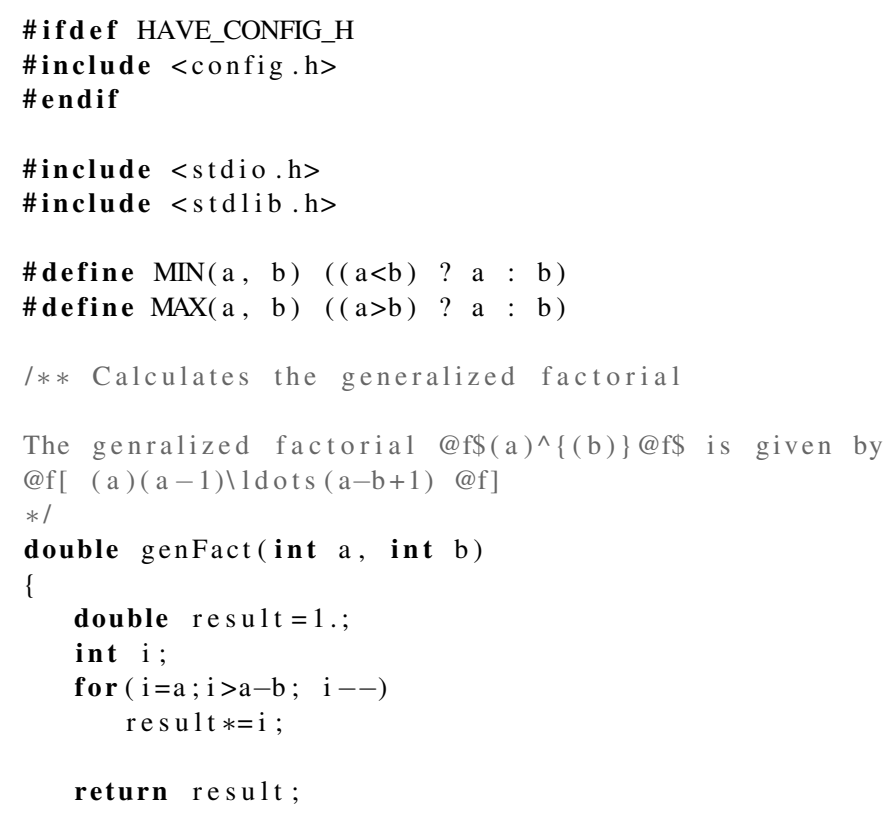


\}

I** Calculates a Gram Polynomial or its derivative

The Gram Polynomial may be calculated according to Gorry, eq. 11 as $@ \mathrm{f}[$

$\mathrm{P}_{-} \mathrm{k}^{\wedge}\{\mathrm{m}, \mathrm{s}\}(\mathrm{i})=\backslash \mathrm{frac}\{2(2 \mathrm{k}-1)\}\{\mathrm{k}(2 \mathrm{~m}-\mathrm{k}+1)\}$

$\backslash 1 \mathrm{eft}\left[\mathrm{i} \mathrm{P}_{-}\{\mathrm{k}-1\}^{\wedge} \mathrm{m}(\mathrm{i})+\mathrm{s} \mathrm{P}_{-}\{\mathrm{k}-1\}^{\wedge}\{\mathrm{m}, \mathrm{s}-1\}(\mathrm{i}) \backslash \mathrm{right}\right]$

@f]

- $\backslash$ frac $\{(\mathrm{k}-1)(2 \mathrm{~m}+\mathrm{k})\}\{\mathrm{k}(2 \mathrm{~m}-\mathrm{k}+)\} \mathrm{P}_{-}\{\mathrm{k}-2\}^{\wedge}\{\mathrm{m}, \mathrm{s}\}$ (i)

*/

double gramPoly (int $\mathrm{i}$, int $\mathrm{m}$, int $\mathrm{k}$, int $\mathrm{s}$ )

\{

double gp ;

double d;

if $(\mathrm{k}>0)$

\{

$\mathrm{d}=\mathrm{k} *(2 * \mathrm{~m}-\mathrm{k}+1)$

$\operatorname{gp}=(($ double $)(4 * \mathrm{k}-2)) / \mathrm{d} *((($ double $) \mathrm{i}) * \operatorname{gramPoly}(\mathrm{i}, \mathrm{m}, \mathrm{k}-1, \mathrm{~s})$

$+(($ double $) \mathrm{s}) * \operatorname{gramPoly}(\mathrm{i}, \mathrm{m}, \mathrm{k}-1, \mathrm{~s}-1))$

- ( (double $)((\mathrm{k}-1) *(2 * \mathrm{~m}+\mathrm{k}))) / \mathrm{d} * \operatorname{gramPoly}(\mathrm{i}, \mathrm{m}, \mathrm{k}-2, \mathrm{~s})$;

return $\mathrm{gp}$;

\}

else

\{

if $(\mathrm{k}==0 \quad \& \& \mathrm{~s}==0)$

return 1. ;

else

return 0 .;

\}

\}

I** Calculates the coefficients for the smoothing filters

Calculates the coefficients for a smoothing filter using the Gorry method.

According to Gorry, Eq. 8, the coefficients are

$@ \mathrm{f}[$

$\mathrm{h}_{-} \mathrm{i}^{\wedge}\{\mathrm{t}, \mathrm{s}\}=\backslash \operatorname{sum}_{-}\{\mathrm{k}=\mathrm{o}\}^{\wedge} \mathrm{n} \backslash \operatorname{frac}\left\{(2 \mathrm{k}+1)(2 \mathrm{~m})^{\wedge}(\mathrm{k})\right\}$

$\left\{(2 \mathrm{~m}+\mathrm{k}+1)^{\wedge}(\mathrm{k}+1)\right\} \mathrm{P}_{-} \mathrm{k}^{\wedge} \mathrm{m}(\mathrm{i}) \mathrm{P}_{-} \mathrm{k}^{\wedge}\{\mathrm{m}, \mathrm{s}\}(\mathrm{t})$

@]

Note that@f $(a)^{\wedge}\{(b)\} @ f \$$ is the generalized factorial function.

$* 1$

double weight (int $\mathrm{i}$, int $\mathrm{t}$, int $\mathrm{m}$, int $\mathrm{n}$, int $\mathrm{s}$ )

\{

int $\mathrm{k}$;

double $\operatorname{sum}=0$.;

for $(\mathrm{k}=0 ; \mathrm{k}<=\mathrm{n} ; \mathrm{k}++)$

\{

sum $+=(($ double $)(2 * \mathrm{k}+1)) * \operatorname{genFact}(2 * \mathrm{~m}, \mathrm{k}) / \operatorname{genFact}(2 * \mathrm{~m}+\mathrm{k}+1, \mathrm{k}+1) *$ $\operatorname{gramPoly}(\mathrm{i}, \mathrm{m}, \mathrm{k}, 0) * \operatorname{gramPoly}(\mathrm{t}, \mathrm{m}, \mathrm{k}, \mathrm{s})$;

\}

return sum;

\}

I** Calculates the smoothed signal or a smoothed derivative of order s 


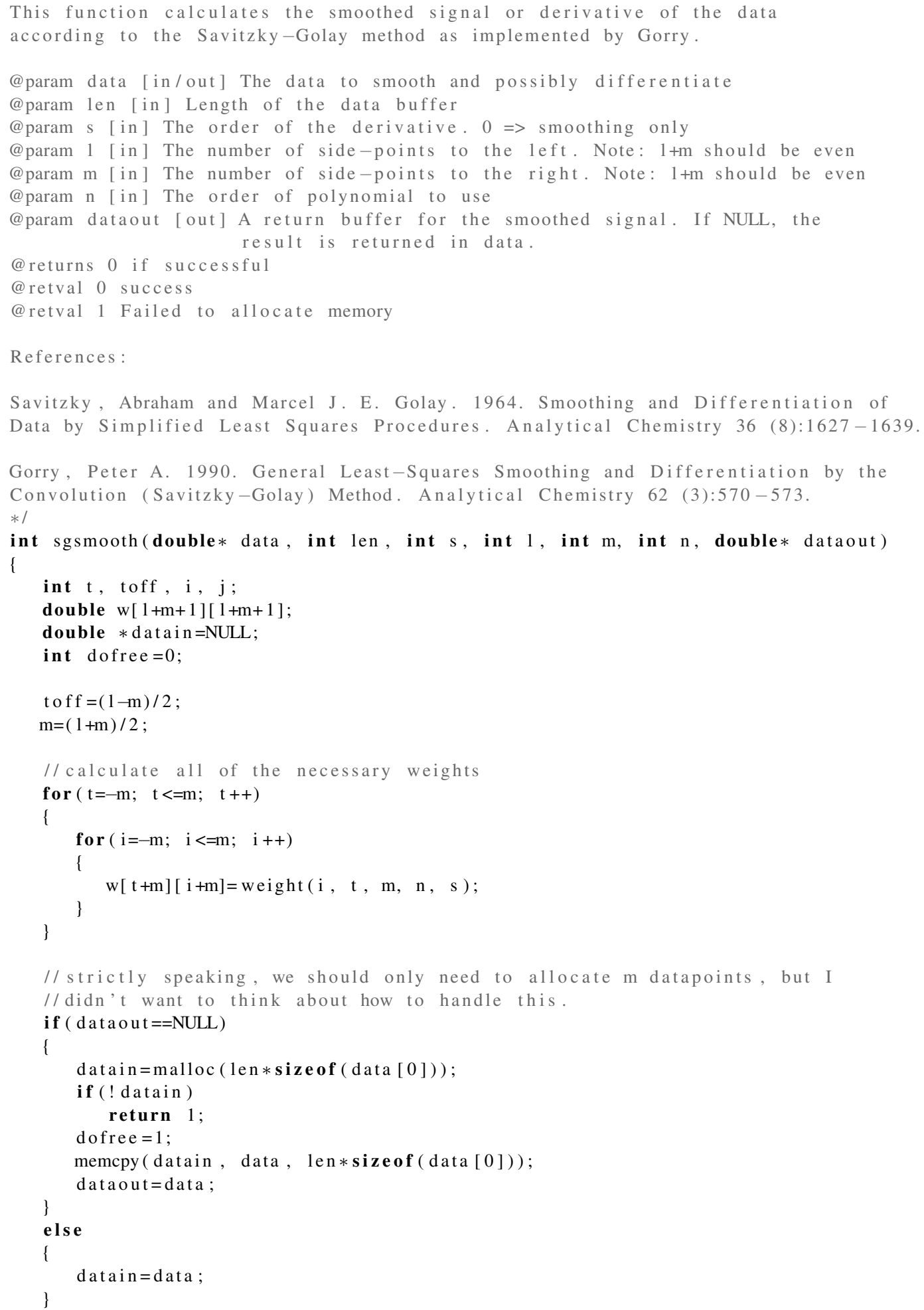




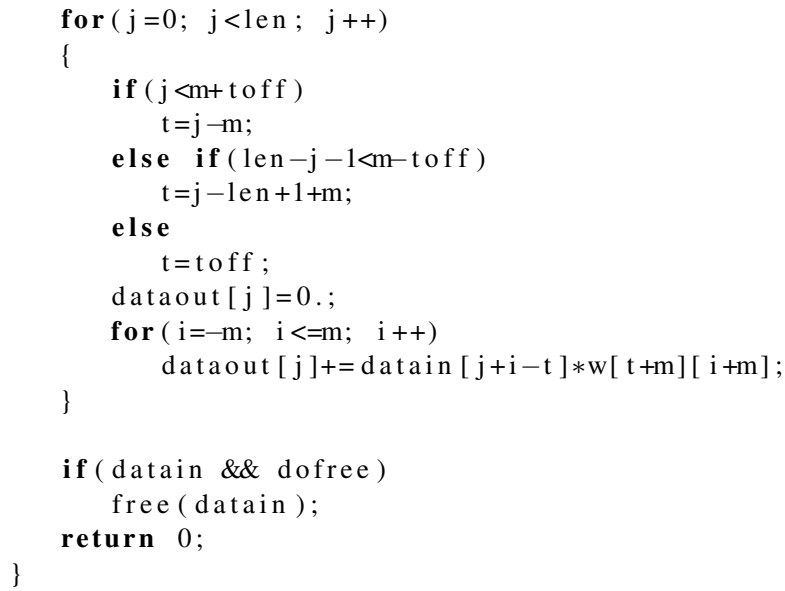

\section{A.3 Cai Plume Neutral Density Model}

The Cai neutral density model ${ }^{4,5}$ is used to estimate the neutral density in the plume of an HET. The HETAnalytical class implements the model.

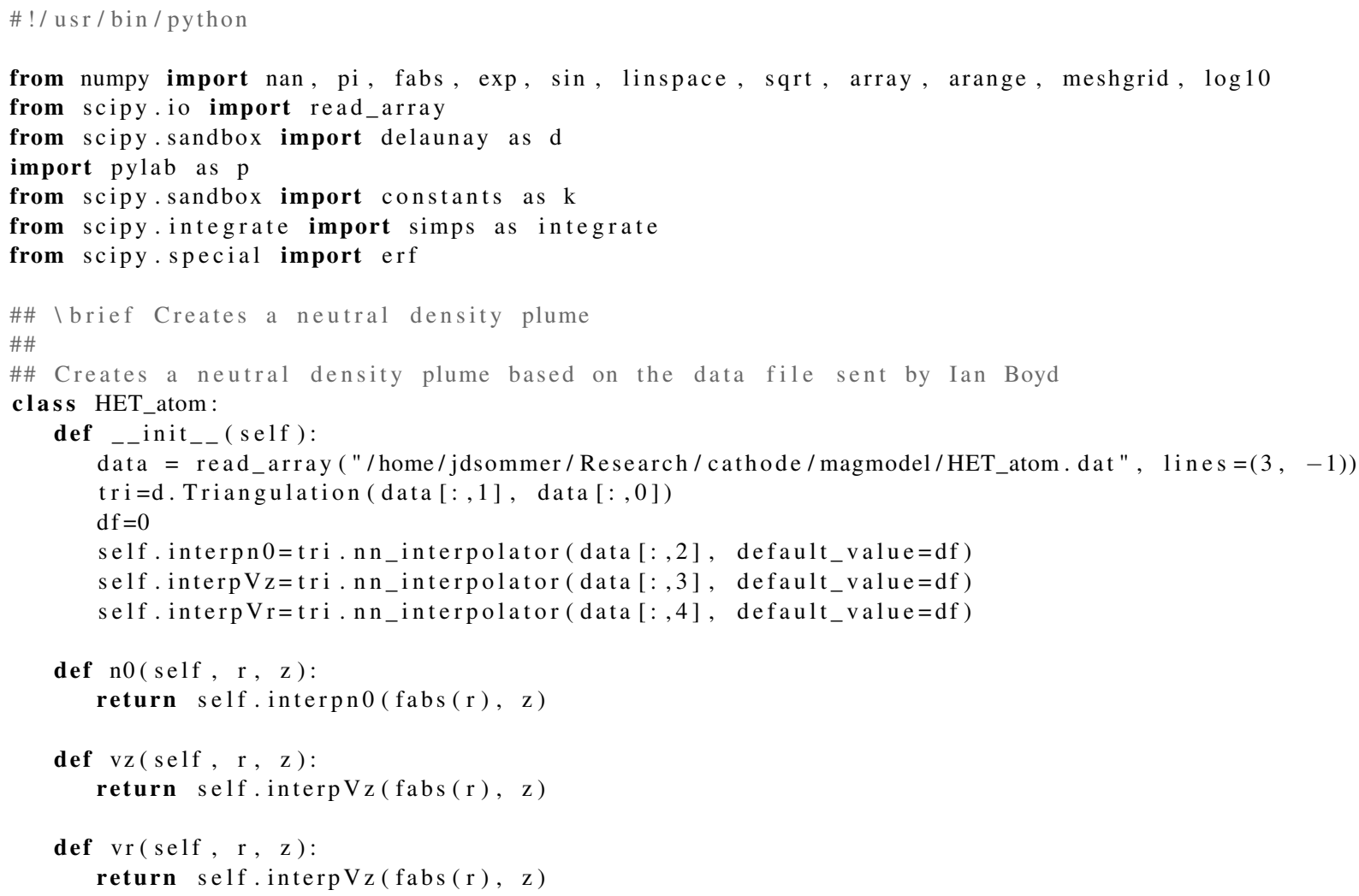




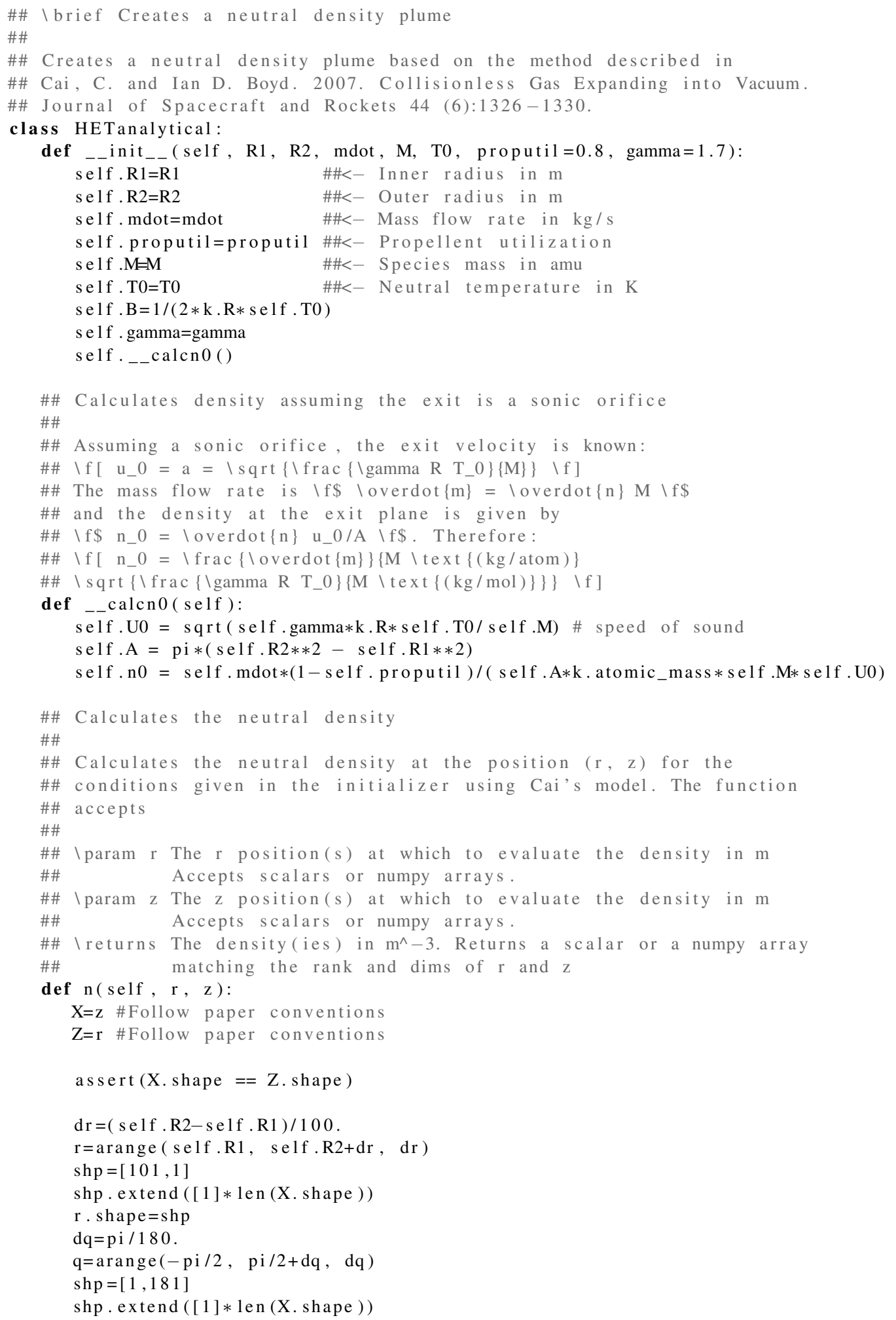




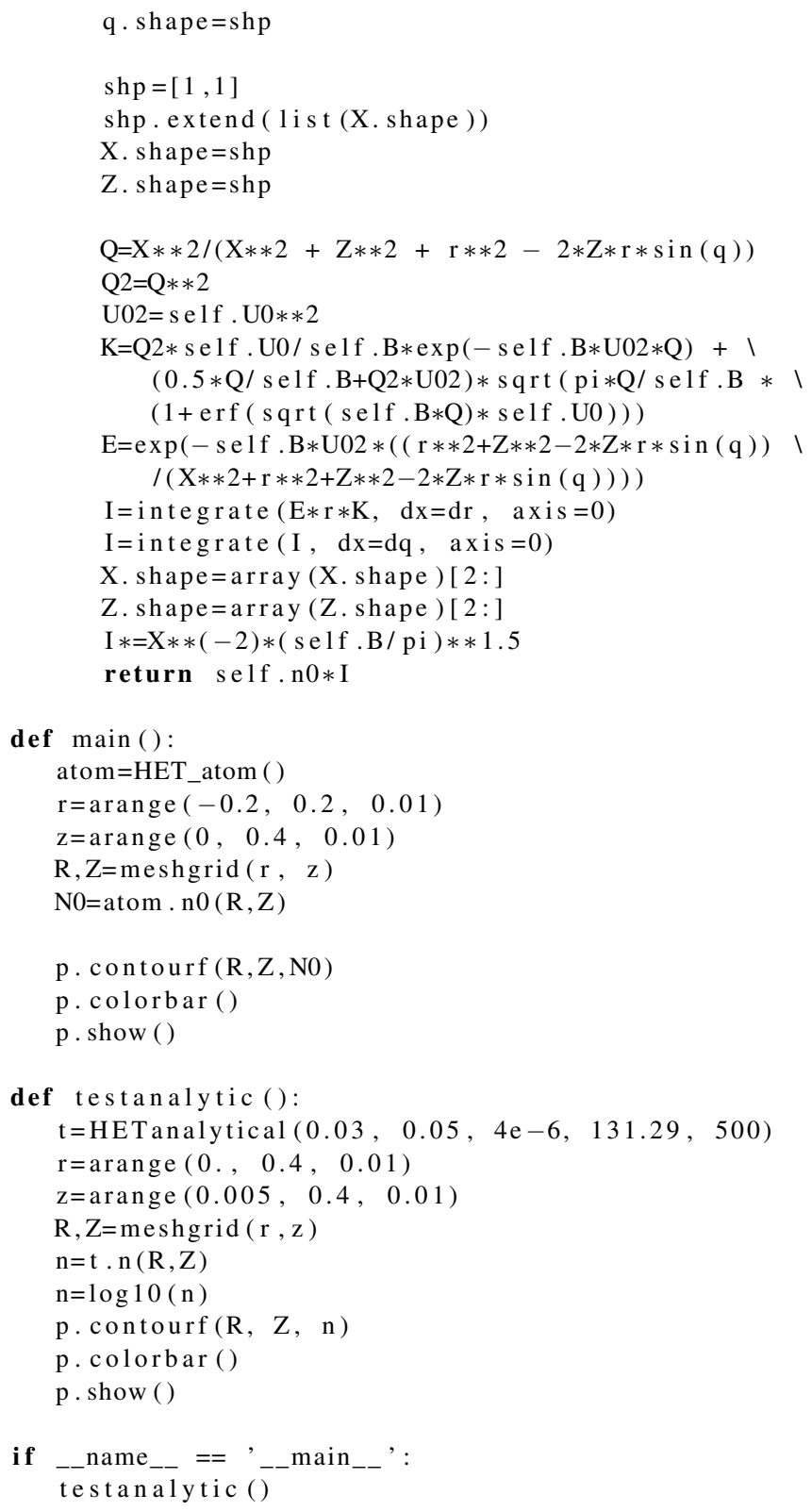

\section{References for Appendix A}

[1] Peterson, E. W. and Talbot, L., "Collisionless Electrostatic Single-Probe and Double-Probe Measurements," AIAA Journal, Vol. 8, No. 12, December 1970, pp. 2215-2219. 
[2] Savitzky, A. and Golay, M. J. E., "Smoothing and Differentiation of Data by Simplified Least Squares Procedures," Analytical Chemistry, Vol. 36, No. 8, July 1964, pp. 1627-1639.

[3] Gorry, P. A., "General Least-Squares Smoothing and Differentiation by the Convolution (Savitzky-Golay) Method," Analytical Chemistry, Vol. 62, No. 3, March 1990, pp. 570-573.

[4] Cai, C. and Boyd, I. D., "Collisionless Gas Expanding into Vacuum," Journal of Spacecraft and Rockets, Vol. 44, No. 6, November 2007, pp. 1326-1330.

[5] Cai, C., Theoretical and Numerical Studies of Plume Flows in Vacuum Chambers, Ph.D. dissertation, University of Michigan, Ann Arbor, MI, 2005. 


\section{Appendix B}

\section{Double Probe Heating}

During the work with xenon probes (see Appendix C) the double probe was moved through a pattern which left it exposed to dense, hot plasma for long periods of time on the order of one minute. The resulting probe heating had a deleterious effect on the resulting measurement. Specifically, it resulted in electron temperature measurements that were large by an order of magnitude. To determine that probe heating was the cause of the error I placed a cool probe inside a hot region of the thruster and took double probe traces every second for two minutes. The probe was cooled by placing it a low density, low temperature region of the plasma, specifically, behind the thruster and as far radially as the motion table would allow: $r=\sim 200 \mathrm{~mm}$.

The results are plotted in Figure B.1 at 15 second intervals. Additonally, the time zero traces are plotted in gray on each of the graphs for reference. The Peterson and Talbot reduction was performed on each trace, and the results printed on the graphs. One can see a general increase in the slope of the tails of the I-V curves as time progresses. This leads to higher estimations of both $T_{e}$ and $n_{e}$ (Figure B.2), which, in turn, lead to higher values for $V_{p}$. The obvious conclusion is that probes should spend a minimum amount of time in dense, hot plasmas or the results will be inaccurate.

Perhaps a more intuitive way of viewing the data is the animation in Figure B.3. This figure shows the complete dataset over the two minute acquisition period at a speed factor of $10 x$. 


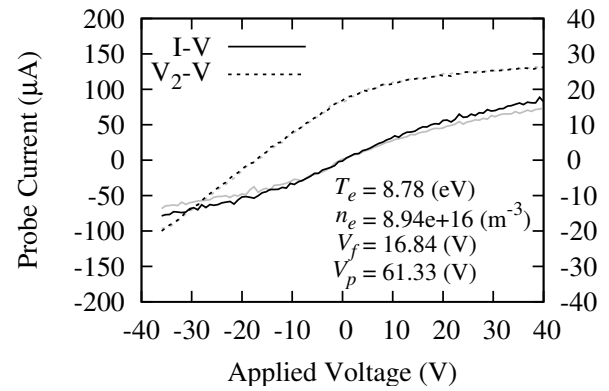

(a) Time $=14 \mathrm{~s}$

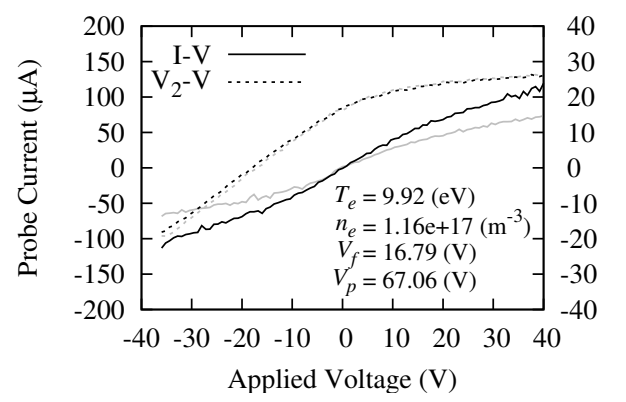

(c) Time $=44 \mathrm{~s}$

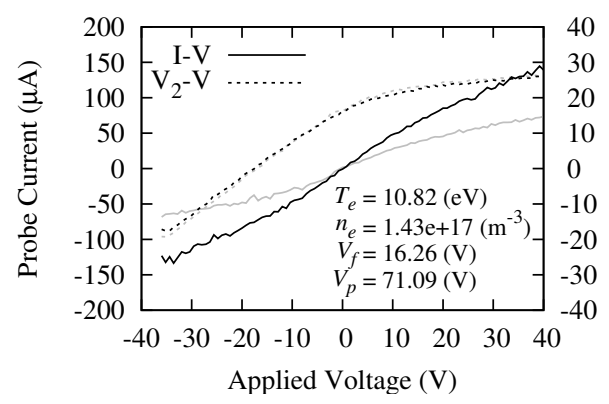

(e) Time $=74 \mathrm{~s}$

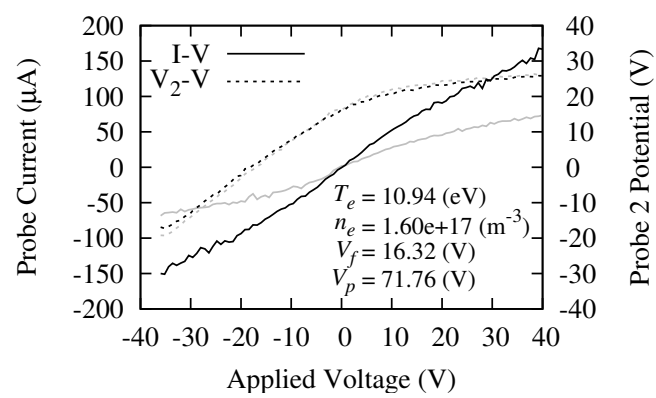

(g) Time $=104 \mathrm{~s}$

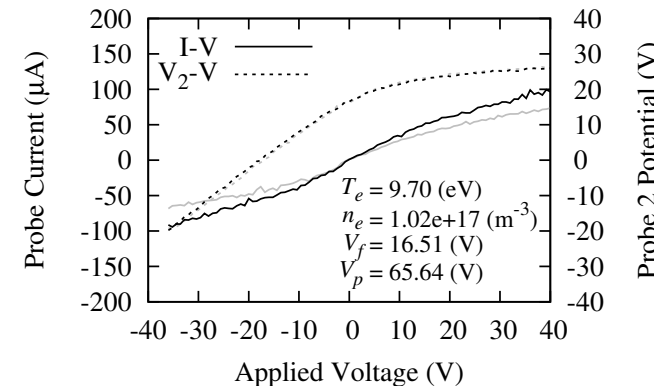

(b) Time $=29 \mathrm{~s}$

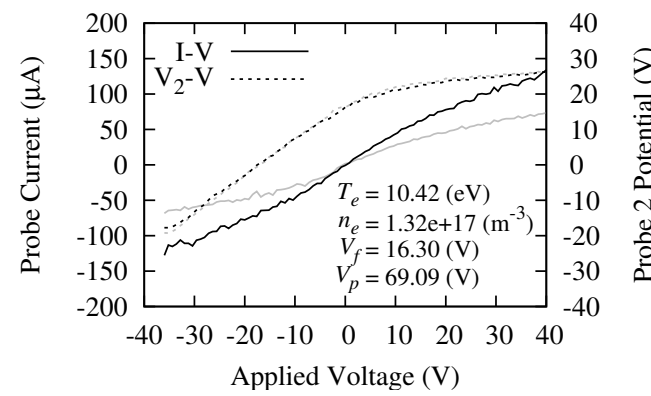

(d) Time $=59 \mathrm{~s}$

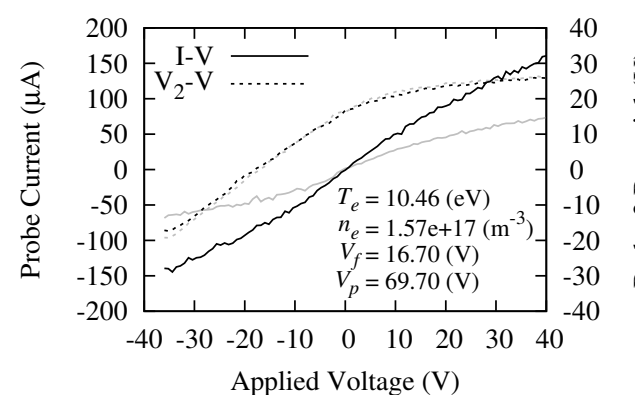

(f) Time $=89 \mathrm{~s}$

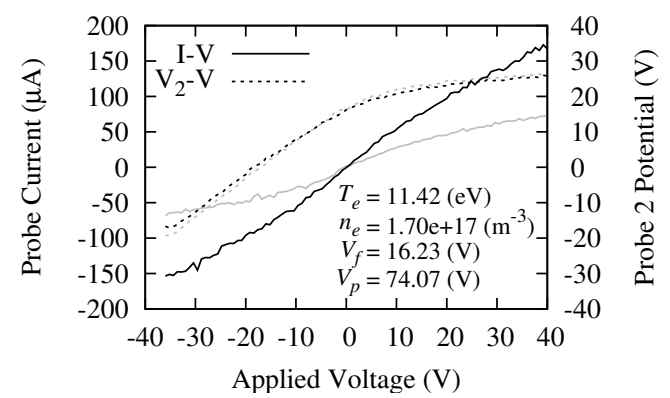

(h) Time $=119 \mathrm{~s}$

Figure B.1: Effect of probe heating on double probe traces 


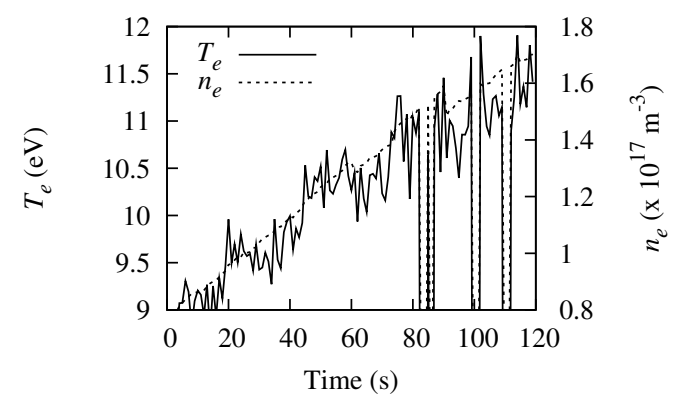

Figure B.2: Effect of probe heating on measurement of plasma parameters

Time $=0 \mathrm{~s}$

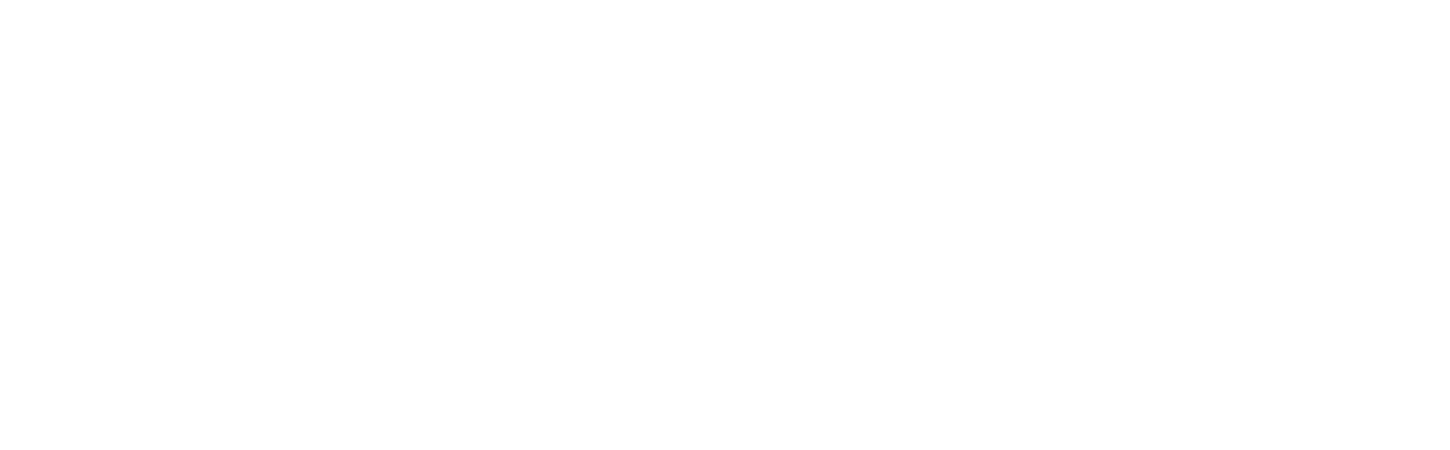

Figure B.3: Animation of the probe traces at 10x speed 


\section{Appendix C}

\section{Plume Properties}

This appendix presents the near-field plume plasma properties for each of the conditions measured. Plume properties were measured while the thruster was operated with both the original outer pole (OOP) and the extended outer pole (EOP) using krypton propellent, and with the OOP when using xenon propellent. Further details of the the experiments can be found in Chapter 8. Each figure presents $T_{e}, n_{e}, V_{f}, V_{p}$, and Cost (the quality of the match of the data to the theoretical model) as described in Sections 5.4.2 and 5.4.3.

\section{Figures}

C.1 Plasma properties for $\mathrm{Kr}$ with the OOP with $\dot{m}_{c}=2 \mathrm{SCCM}, r=50 \mathrm{~mm} 186$

C.2 Plasma properties for Kr with the OOP with $\dot{m}_{c}=2 \mathrm{SCCM}, r=60 \mathrm{~mm} 187$

C.3 Plasma properties for $\mathrm{Kr}$ with the OOP with $\dot{m}_{c}=2 \mathrm{SCCM}, r=80 \mathrm{~mm} 188$

C.4 Plasma properties for $\mathrm{Kr}$ with the OOP with $\dot{m}_{c}=2 \mathrm{SCCM}, r=$ $100 \mathrm{~mm} \ldots \ldots \ldots \ldots . \ldots \ldots$

C.5 Plasma properties for Kr with the OOP with $\dot{m}_{c}=5 \mathrm{SCCM}, r=50 \mathrm{~mm} 190$

C.6 Plasma properties for Kr with the OOP with $\dot{m}_{c}=5 \mathrm{SCCM}, r=60 \mathrm{~mm} 191$

C.7 Plasma properties for Kr with the OOP with $\dot{m}_{c}=5 \mathrm{SCCM}, r=80 \mathrm{~mm} 192$

C.8 Plasma properties for $\mathrm{Kr}$ with the OOP with $\dot{m}_{c}=5 \mathrm{SCCM}, r=$ $100 \mathrm{~mm} \ldots \ldots \ldots \ldots \ldots$

C.9 Plasma properties for Kr with the OOP with $\dot{m}_{c}=5$ SCCM, $r=$ $120 \mathrm{~mm} \ldots \ldots \ldots \ldots \ldots$. . . . . . . . . . 194

C.10 Plasma properties for $\mathrm{Kr}$ with the OOP with $\dot{m}_{c}=5 \mathrm{SCCM}, r=$ $200 \mathrm{~mm} \ldots \ldots \ldots \ldots \ldots$

C.11 Plasma properties for $\mathrm{Kr}$ with the OOP with $\dot{m}_{c}=10 \mathrm{SCCM}, r=$ $50 \mathrm{~mm} \ldots \ldots \ldots \ldots \ldots \ldots$ 
C.12 Plasma properties for $\mathrm{Kr}$ with the OOP with $\dot{m}_{c}=10 \mathrm{SCCM}, r=$ $60 \mathrm{~mm} \ldots \ldots \ldots \ldots \ldots$. . . . . . . . . . . 197

C.13 Plasma properties for $\mathrm{Kr}$ with the OOP with $\dot{m}_{c}=10 \mathrm{SCCM}, r=$ $80 \mathrm{~mm} \ldots \ldots \ldots \ldots \ldots \ldots$

C.14 Plasma properties for $\mathrm{Kr}$ with the OOP with $\dot{m}_{c}=10 \mathrm{SCCM}, r=$ $100 \mathrm{~mm} \ldots \ldots \ldots \ldots 19 . \ldots \ldots$

C.15 Plasma properties for Kr with the OOP with $\dot{m}_{c}=10$ SCCM, $r=$ $120 \mathrm{~mm} \ldots \ldots \ldots \ldots 20 \ldots \ldots \ldots$

C.16 Plasma properties for $\mathrm{Kr}$ with the OOP with $\dot{m}_{c}=10 \mathrm{SCCM}, r=$

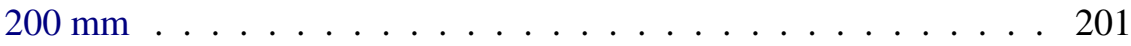

C.17 Plasma properties for $\mathrm{Kr}$ with the EOP with $\dot{m}_{c}=2 \mathrm{SCCM}, r=50 \mathrm{~mm} 204$

C.18 Plasma properties for $\mathrm{Kr}$ with the EOP with $\dot{m}_{c}=2 \mathrm{SCCM}, r=60 \mathrm{~mm} 205$

C.19 Plasma properties for Kr with the EOP with $\dot{m}_{c}=2 \mathrm{SCCM}, r=80 \mathrm{~mm} 206$

C.20 Plasma properties for Kr with the EOP with $\dot{m}_{c}=2$ SCCM, $r=100 \mathrm{~mm} 207$

C.21 Plasma properties for Kr with the EOP with $\dot{m}_{c}=2 \mathrm{SCCM}, r=120 \mathrm{~mm} 208$

C.22 Plasma properties for $\mathrm{Kr}$ with the EOP with $\dot{m}_{c}=5 \mathrm{SCCM}, r=50 \mathrm{~mm} 209$

C.23 Plasma properties for Kr with the EOP with $\dot{m}_{c}=5 \mathrm{SCCM}, r=60 \mathrm{~mm} 210$

C.24 Plasma properties for $\mathrm{Kr}$ with the EOP with $\dot{m}_{c}=5 \mathrm{SCCM}, r=80 \mathrm{~mm} 211$

C. 25 Plasma properties for Kr with the EOP with $\dot{m}_{c}=5 \mathrm{SCCM}, r=100 \mathrm{~mm} 212$

C.26 Plasma properties for Kr with the EOP with $\dot{m}_{c}=5 \mathrm{SCCM}, r=120 \mathrm{~mm} 213$

C.27 Plasma properties for Kr with the EOP with $\dot{m}_{c}=5 \mathrm{SCCM}, r=200 \mathrm{~mm} 214$

C.28 Plasma properties for Kr with the EOP with $\dot{m}_{c}=10 \mathrm{SCCM}, r=50 \mathrm{~mm} 215$

C.29 Plasma properties for Kr with the EOP with $\dot{m}_{c}=10 \mathrm{SCCM}, r=60 \mathrm{~mm} 216$

C.30 Plasma properties for Kr with the EOP with $\dot{m}_{c}=10 \mathrm{SCCM}, r=80 \mathrm{~mm} 217$

C.31 Plasma properties for $\mathrm{Kr}$ with the EOP with $\dot{m}_{c}=10 \mathrm{SCCM}, r=$ $100 \mathrm{~mm} \ldots \ldots \ldots \ldots \ldots \ldots \ldots$

C.32 Plasma properties for $\mathrm{Kr}$ with the EOP with $\dot{m}_{c}=10 \mathrm{SCCM}, r=$ $120 \mathrm{~mm} \ldots \ldots \ldots \ldots \ldots \ldots \ldots$

C.33 Plasma properties for $\mathrm{Kr}$ with the EOP with $\dot{m}_{c}=10 \mathrm{SCCM}, r=$ $200 \mathrm{~mm} \ldots \ldots \ldots \ldots \ldots \ldots$

C.34 Plasma properties for Xe with $\dot{m}_{c}=2 \mathrm{SCCM}, r=60 \mathrm{~mm} \ldots \ldots . .222$

C.35 Plasma properties for Xe with $\dot{m}_{c}=2 \mathrm{SCCM}, r=80 \mathrm{~mm}$. . . . . . 223

C.36 Plasma properties for Xe with $\dot{m}_{c}=2 \mathrm{SCCM}, r=120 \mathrm{~mm} \quad \ldots$. . . 224

C.37 Plasma properties for Xe with $\dot{m}_{c}=5 \mathrm{SCCM}, r=60 \mathrm{~mm} \ldots \ldots .225$

C.38 Plasma properties for Xe with $\dot{m}_{c}=5 \mathrm{SCCM}, r=80 \mathrm{~mm} \ldots \ldots . .226$ 
C.39 Plasma properties for Xe with $\dot{m}_{c}=5 \mathrm{SCCM}, r=120 \mathrm{~mm} \ldots \ldots 227$

C.40 Plasma properties for Xe with $\dot{m}_{c}=10 \mathrm{SCCM}, r=60 \mathrm{~mm} \ldots 228$

C.41 Plasma properties for Xe with $\dot{m}_{c}=10 \mathrm{SCCM}, r=80 \mathrm{~mm} \ldots$. . . 229

C.42 Plasma properties for Xe with $\dot{m}_{c}=10 \mathrm{SCCM}, r=120 \mathrm{~mm} \quad \ldots .230$

\section{C.1 Kr OOP}

These data were acquired while operating the HET at $V_{d}=250 \mathrm{~V}$ with krypton and the original outer pole. $I_{\text {mag }}$ was 2.5 A. 

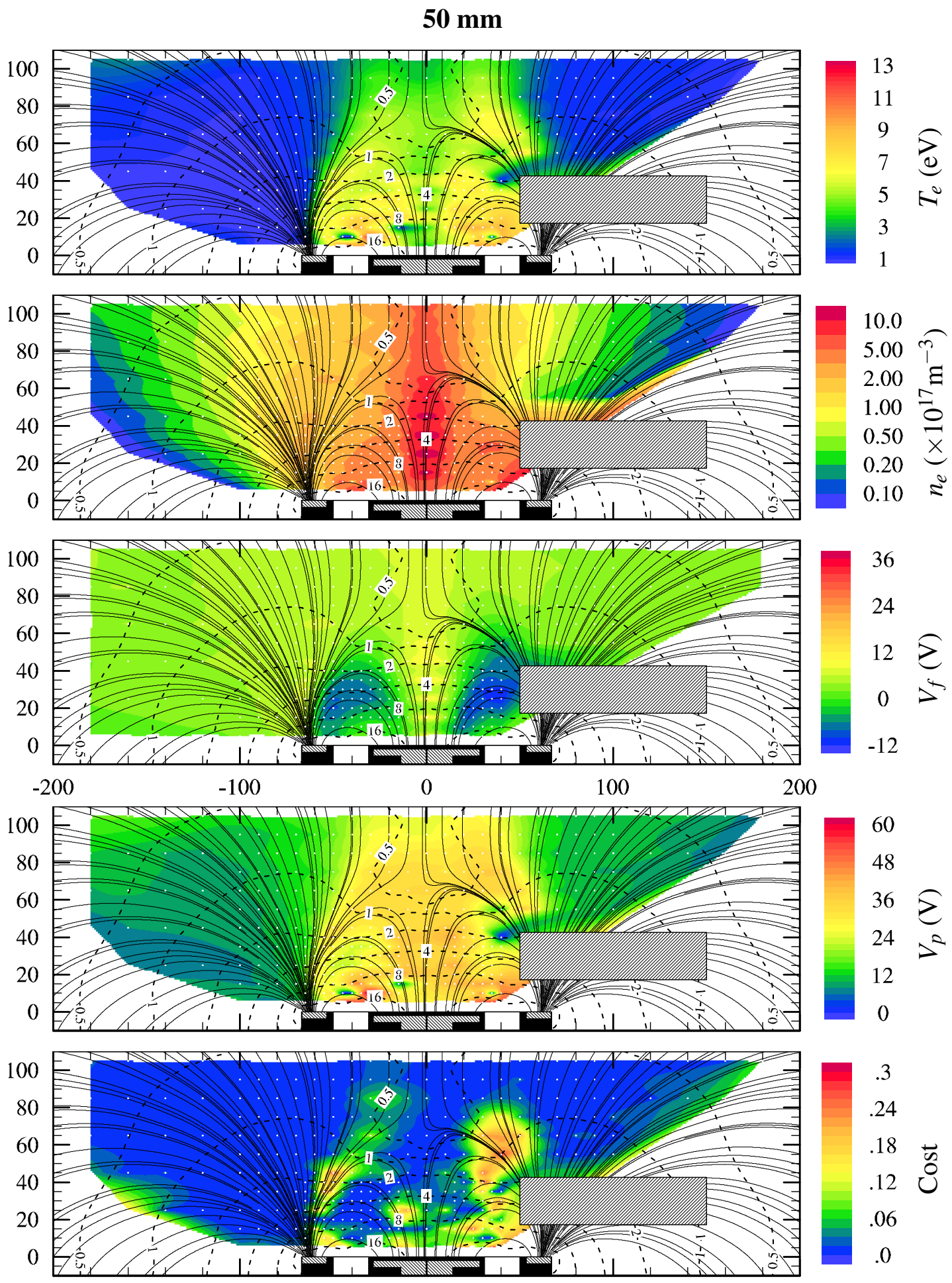

Figure C.1: Plasma properties for $\mathrm{Kr}$ with the OOP with $\dot{m}_{c}=2 \mathrm{SCCM}, r=50 \mathrm{~mm}$ 
$60 \mathrm{~mm}$
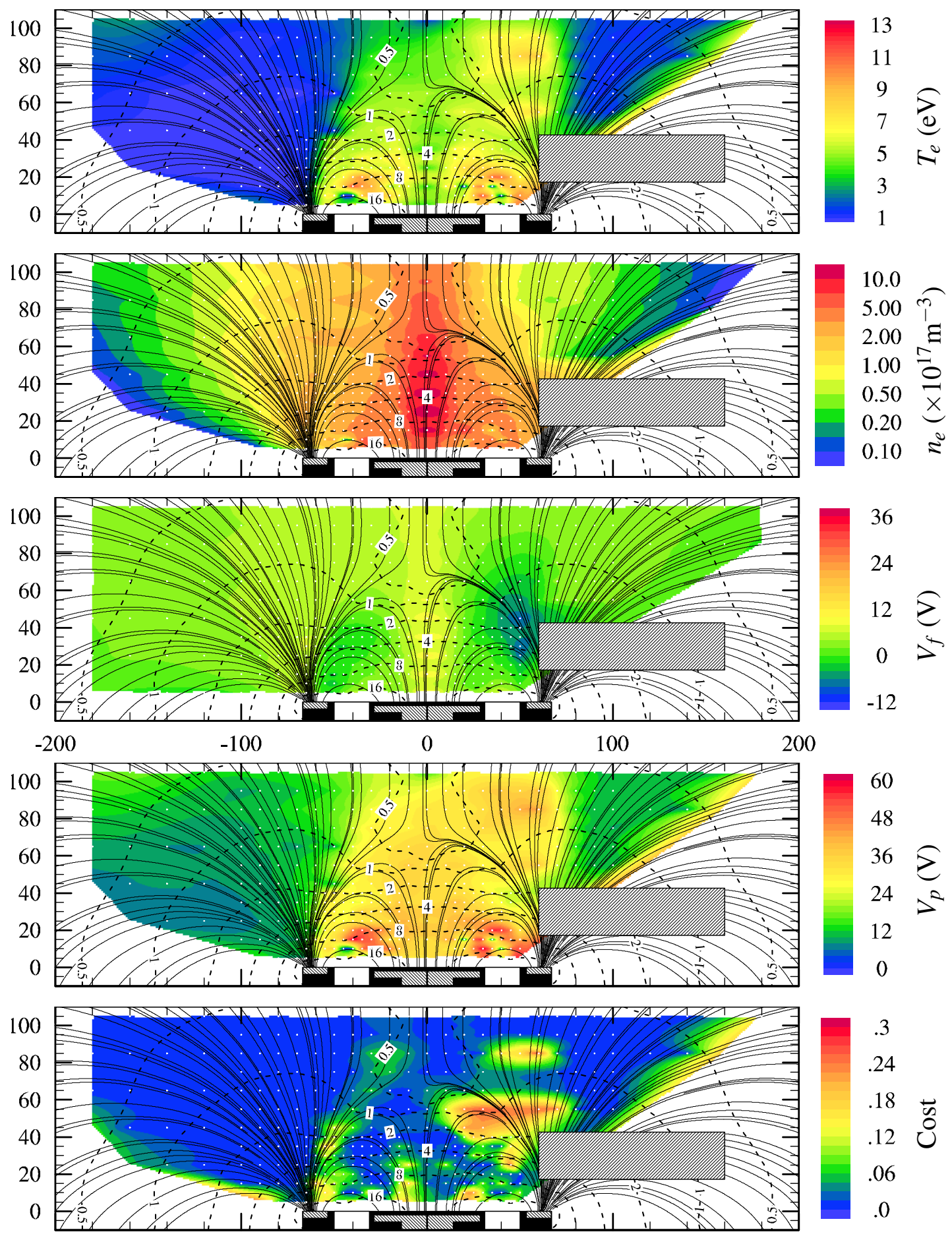

Figure C.2: Plasma properties for Kr with the OOP with $\dot{m}_{c}=2 \mathrm{SCCM}, r=60 \mathrm{~mm}$ 


\section{$80 \mathrm{~mm}$}
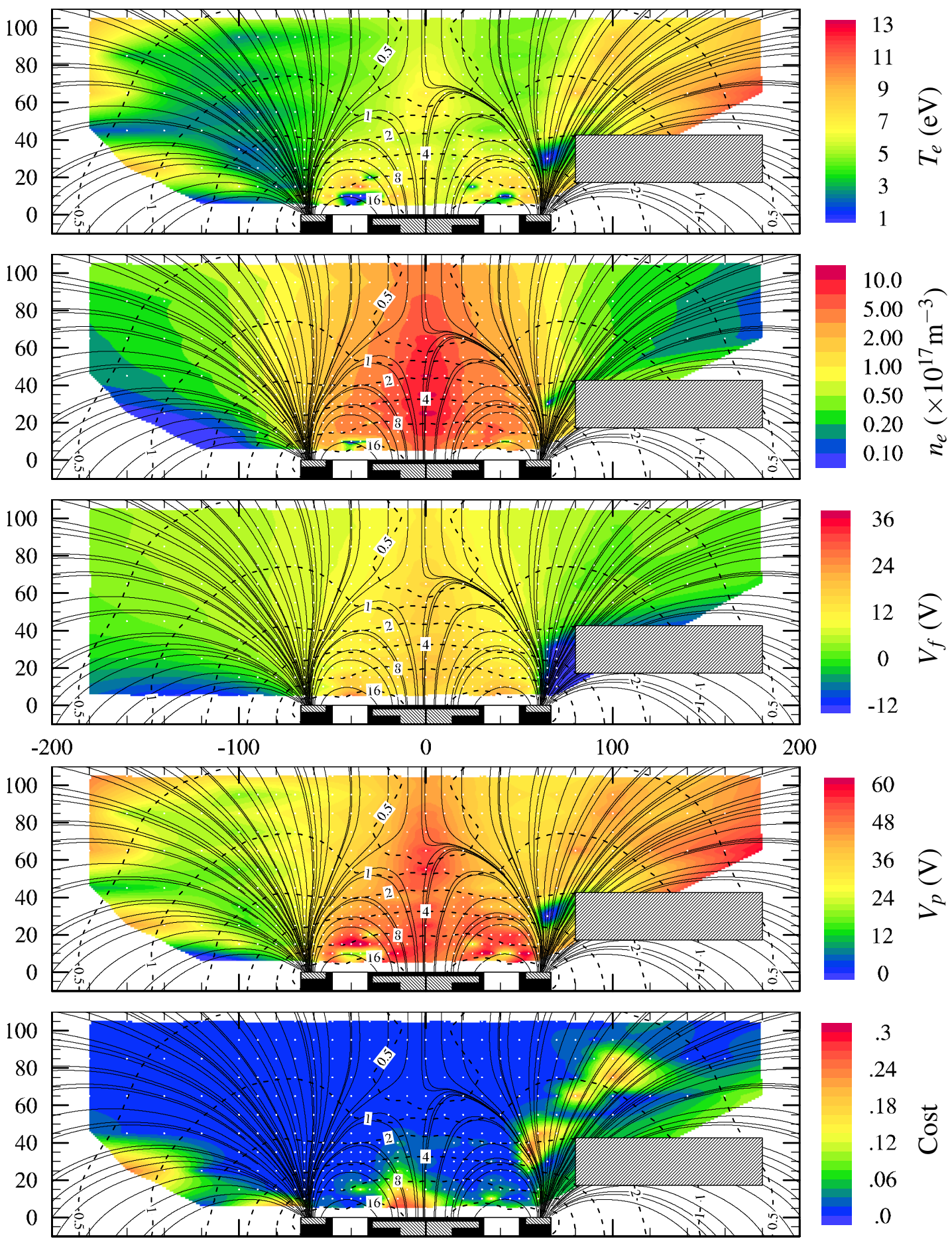

Figure C.3: Plasma properties for Kr with the OOP with $\dot{m}_{c}=2 \mathrm{SCCM}, r=80 \mathrm{~mm}$ 
$100 \mathrm{~mm}$
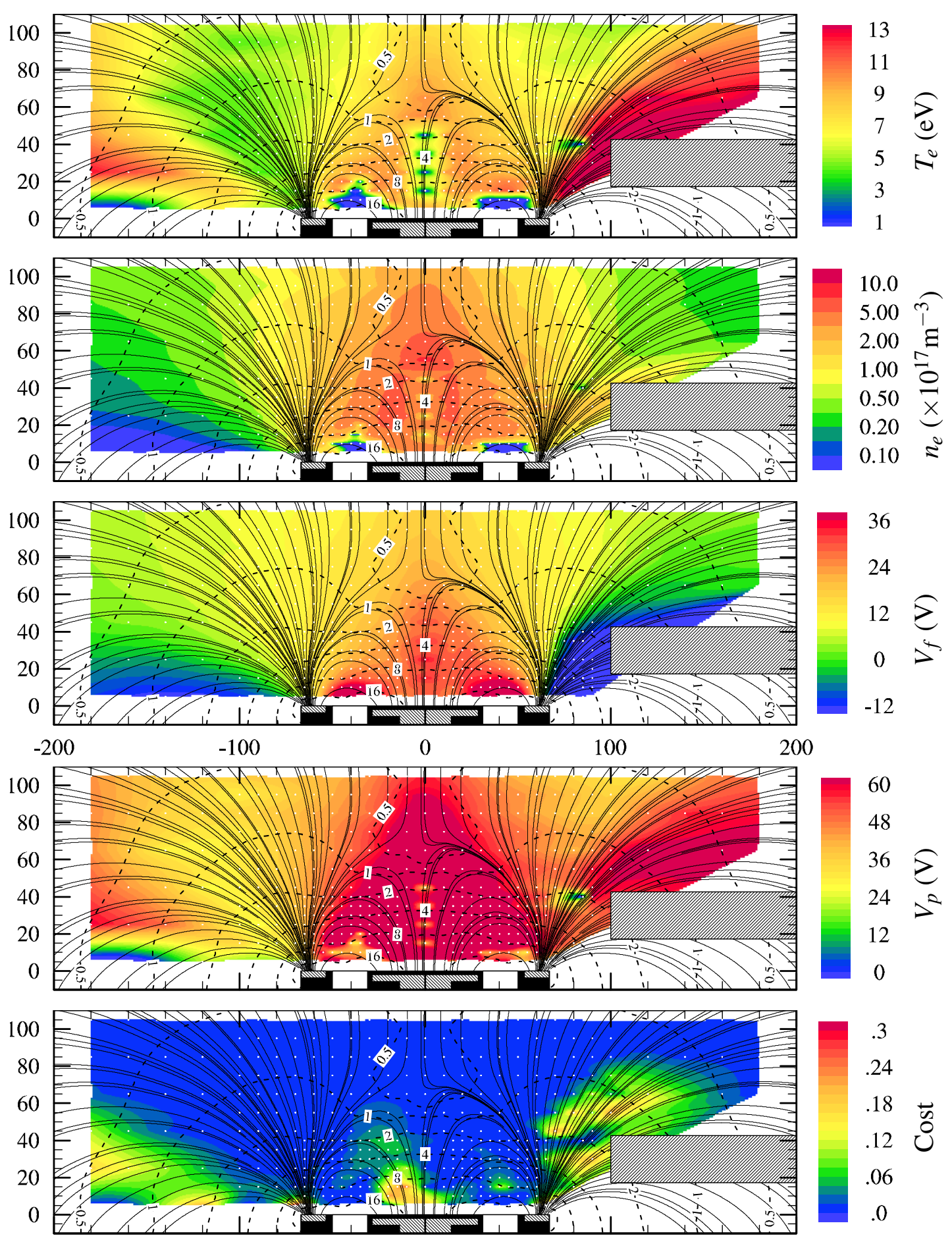

Figure C.4: Plasma properties for Kr with the OOP with $\dot{m}_{c}=2 \mathrm{SCCM}, r=100 \mathrm{~mm}$ 

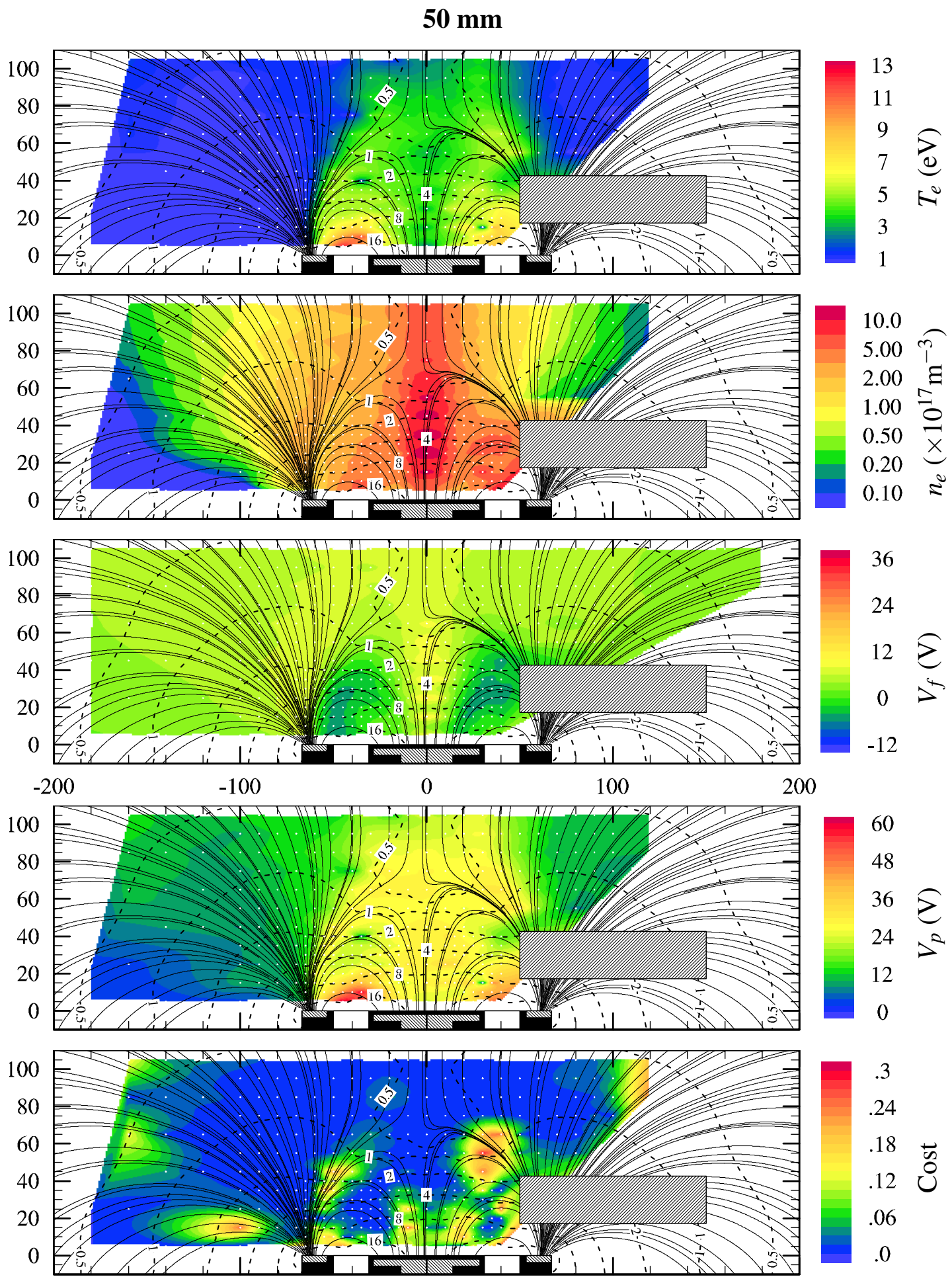

Figure C.5: Plasma properties for $\mathrm{Kr}$ with the OOP with $\dot{m}_{c}=5 \mathrm{SCCM}, r=50 \mathrm{~mm}$ 
$60 \mathrm{~mm}$
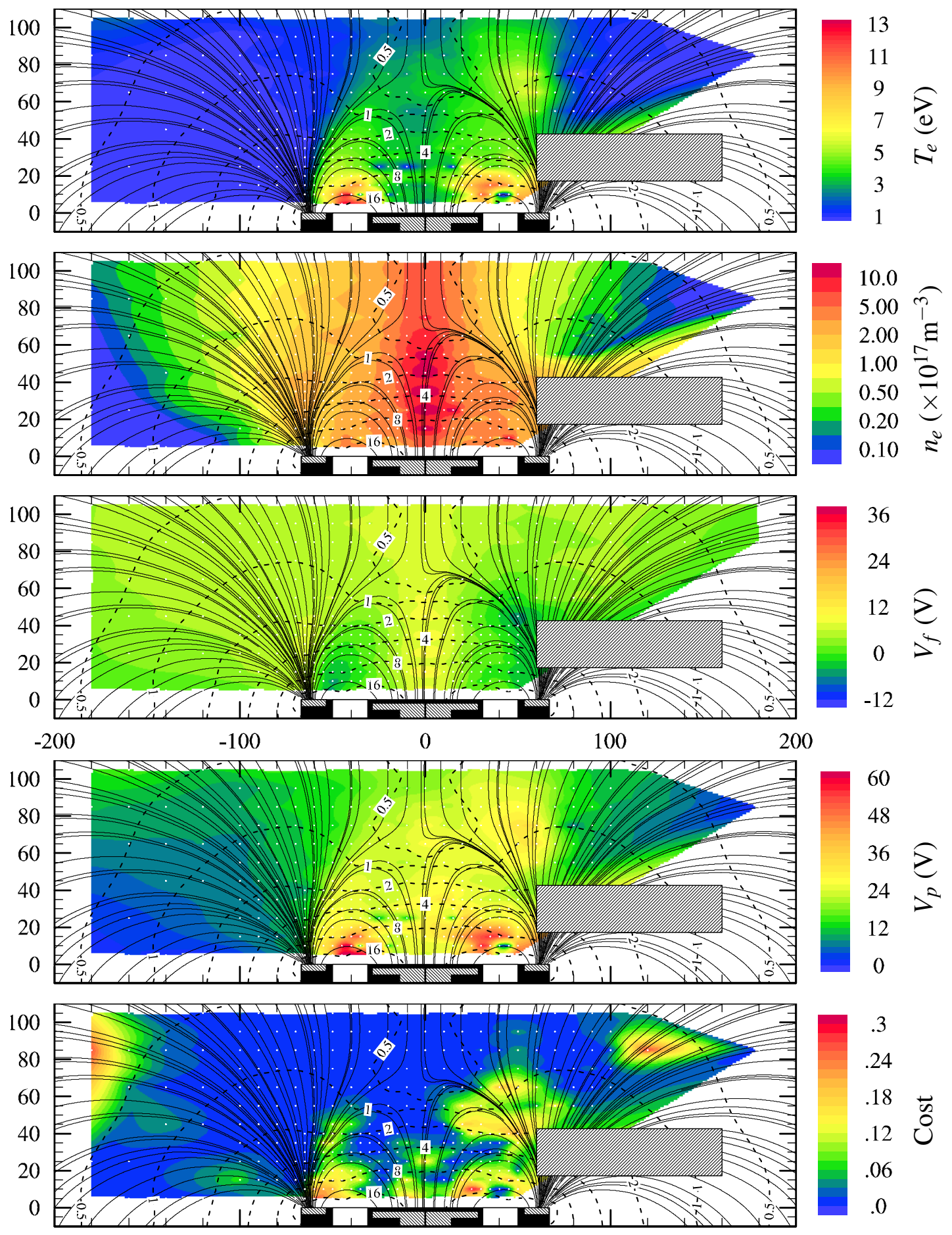

Figure C.6: Plasma properties for Kr with the OOP with $\dot{m}_{c}=5 \mathrm{SCCM}, r=60 \mathrm{~mm}$ 
$80 \mathrm{~mm}$
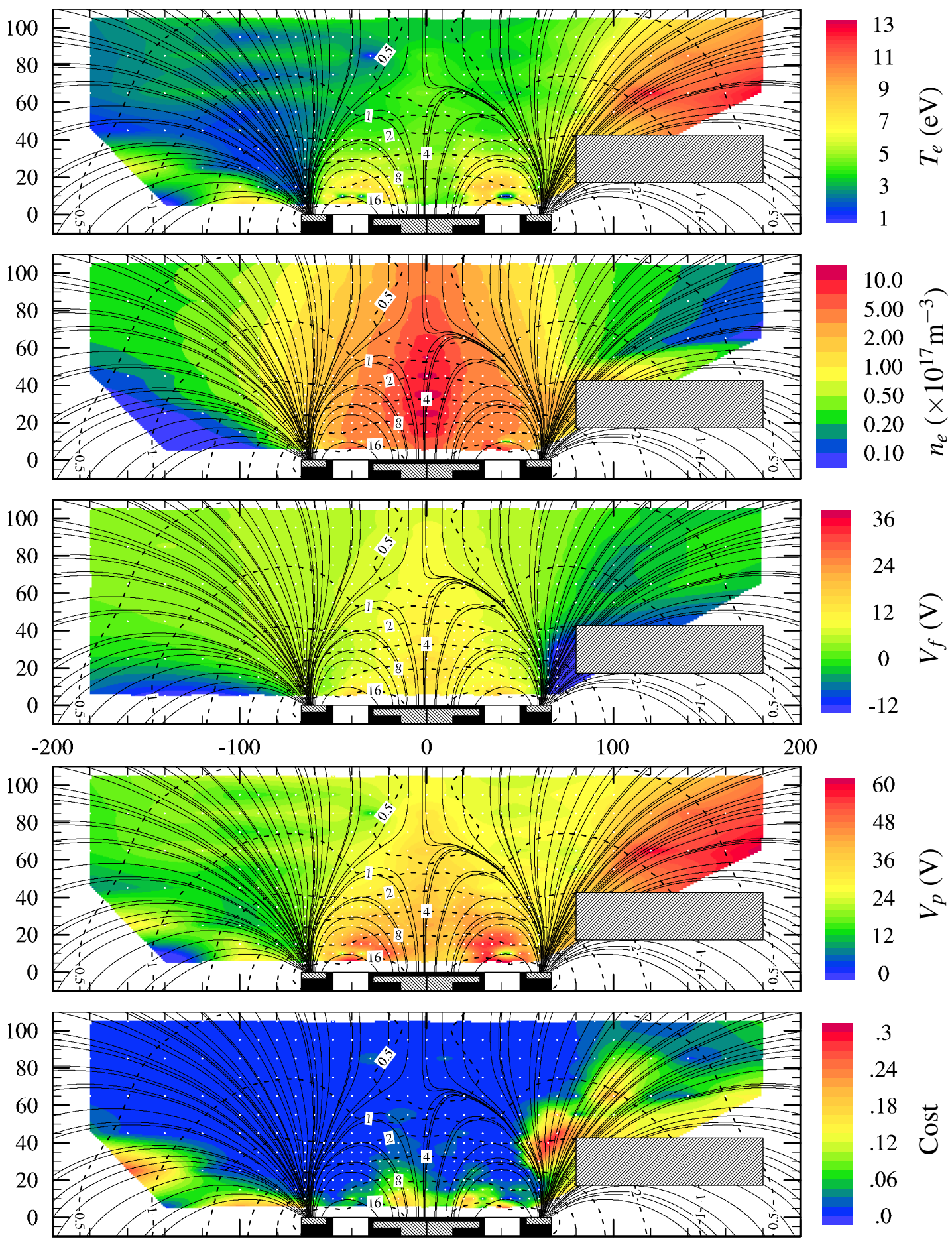

Figure C.7: Plasma properties for $\mathrm{Kr}$ with the OOP with $\dot{m}_{c}=5 \mathrm{SCCM}, r=80 \mathrm{~mm}$ 
$100 \mathrm{~mm}$
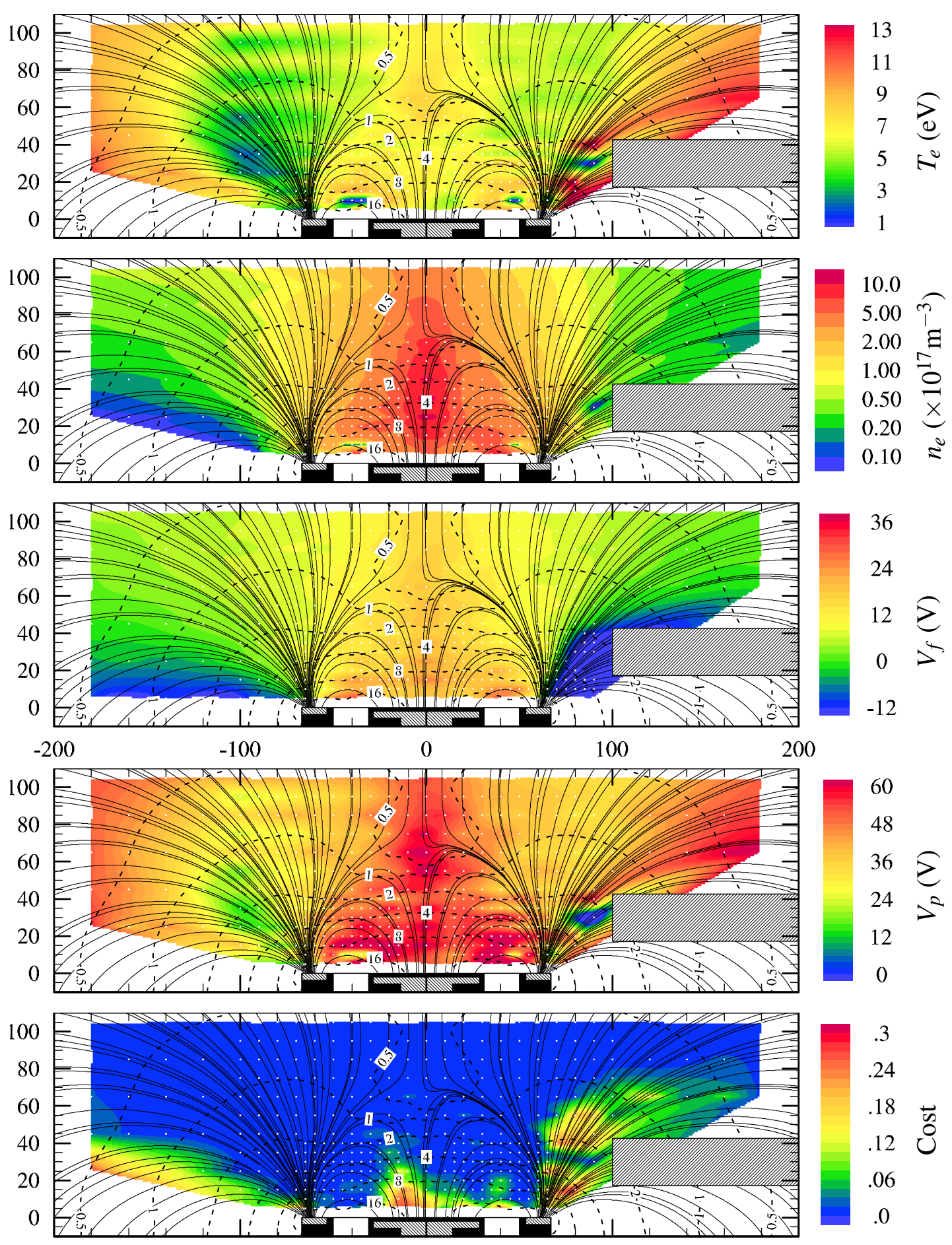

Figure C.8: Plasma properties for Kr with the OOP with $\dot{m}_{c}=5 \mathrm{SCCM}, r=100 \mathrm{~mm}$ 


\section{$120 \mathrm{~mm}$}
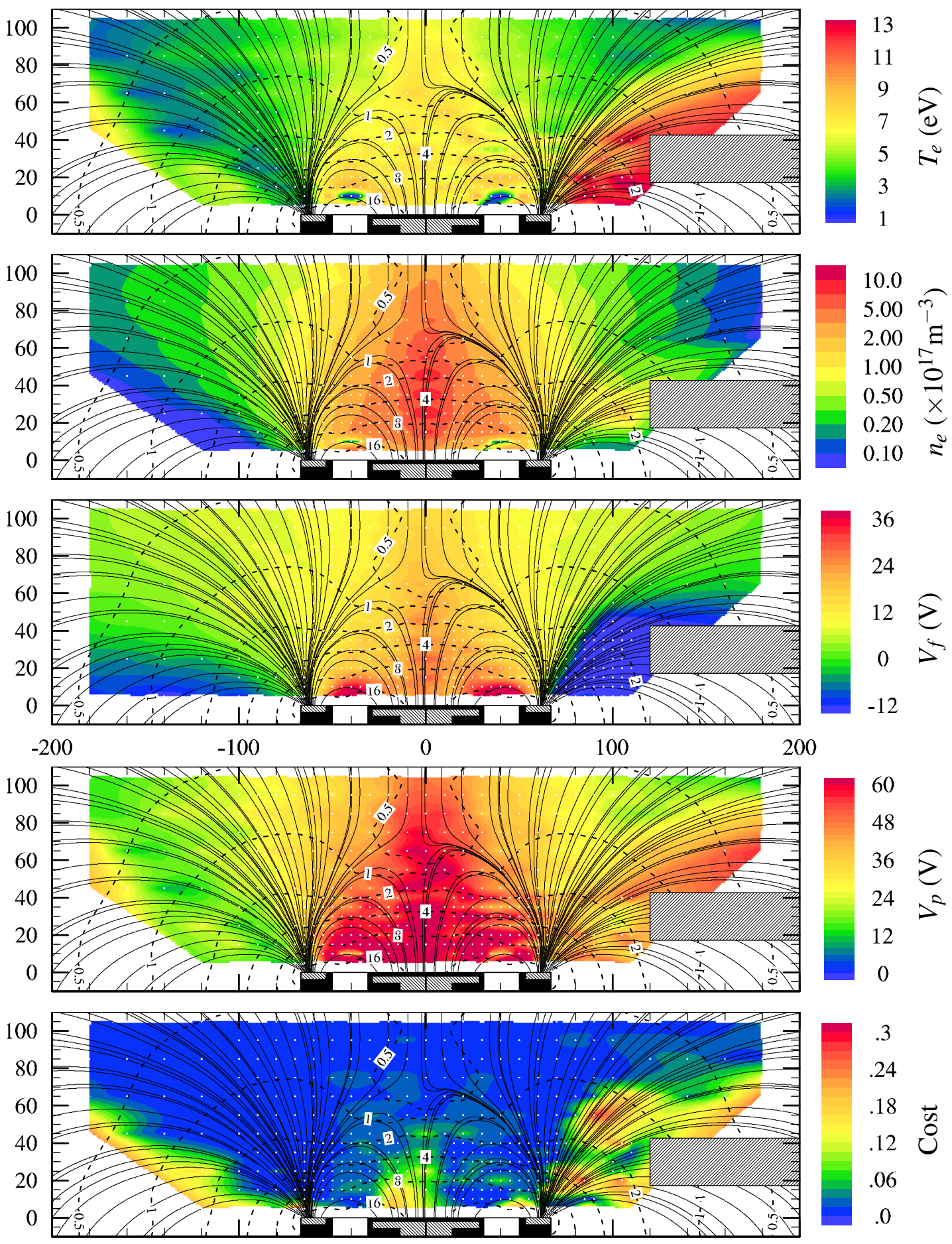

Figure C.9: Plasma properties for $\mathrm{Kr}$ with the OOP with $\dot{m}_{c}=5 \mathrm{SCCM}, r=120 \mathrm{~mm}$ 
$200 \mathrm{~mm}$
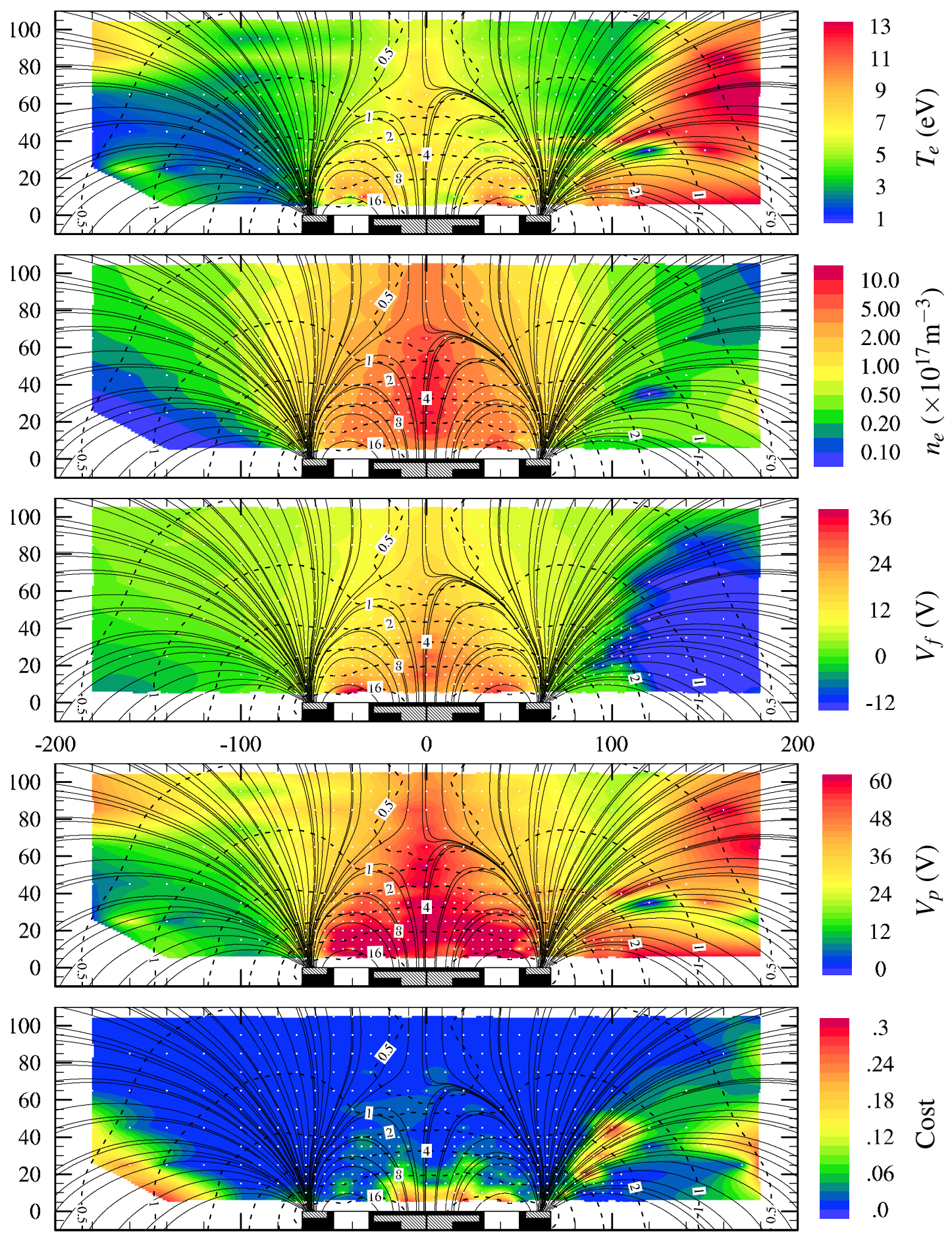

Figure C.10: Plasma properties for Kr with the OOP with $\dot{m}_{c}=5 \mathrm{SCCM}, r=200 \mathrm{~mm}$ 

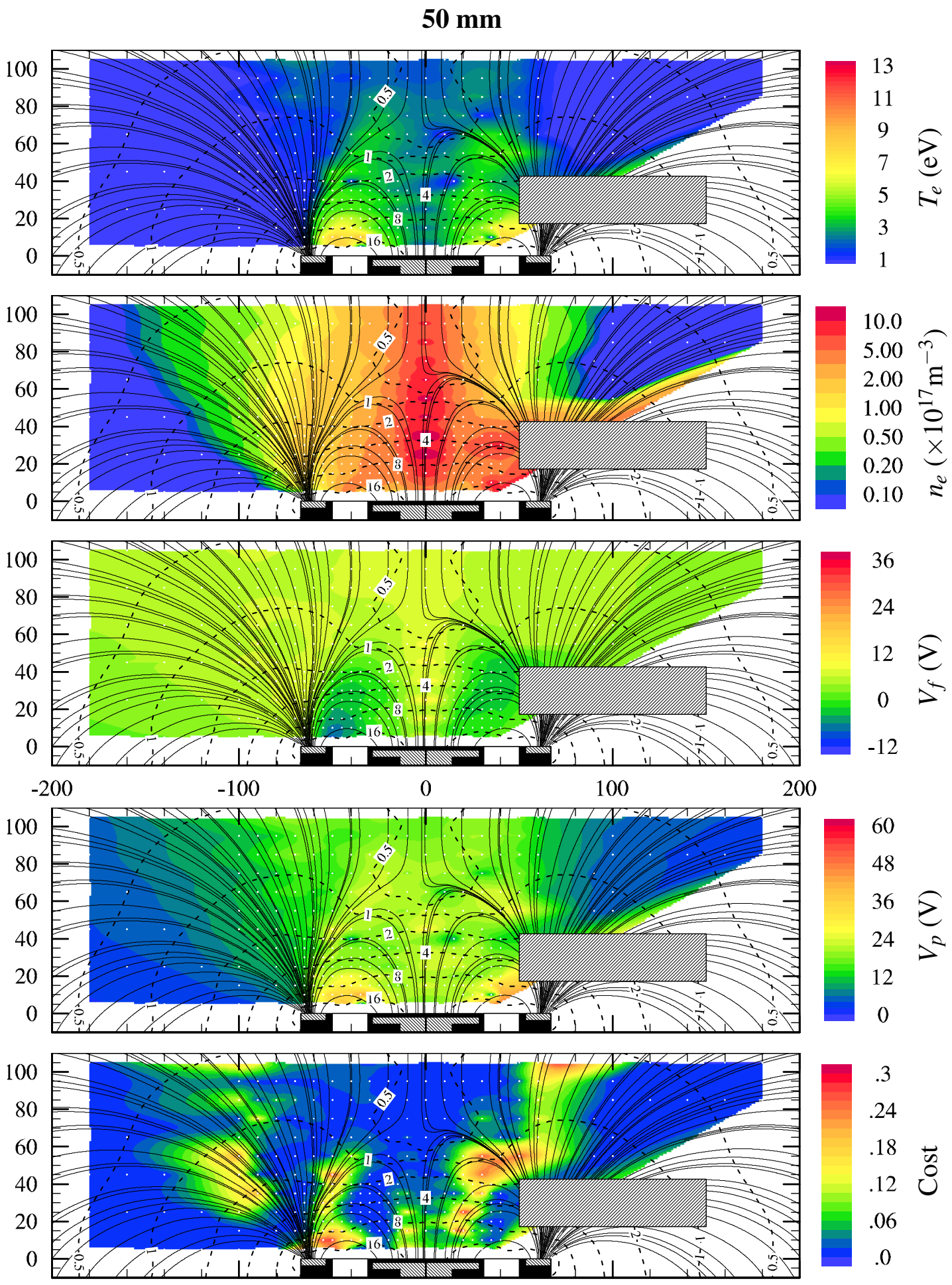

Figure C.11: Plasma properties for $\mathrm{Kr}$ with the OOP with $\dot{m}_{c}=10 \mathrm{SCCM}, r=50 \mathrm{~mm}$ 
$60 \mathrm{~mm}$
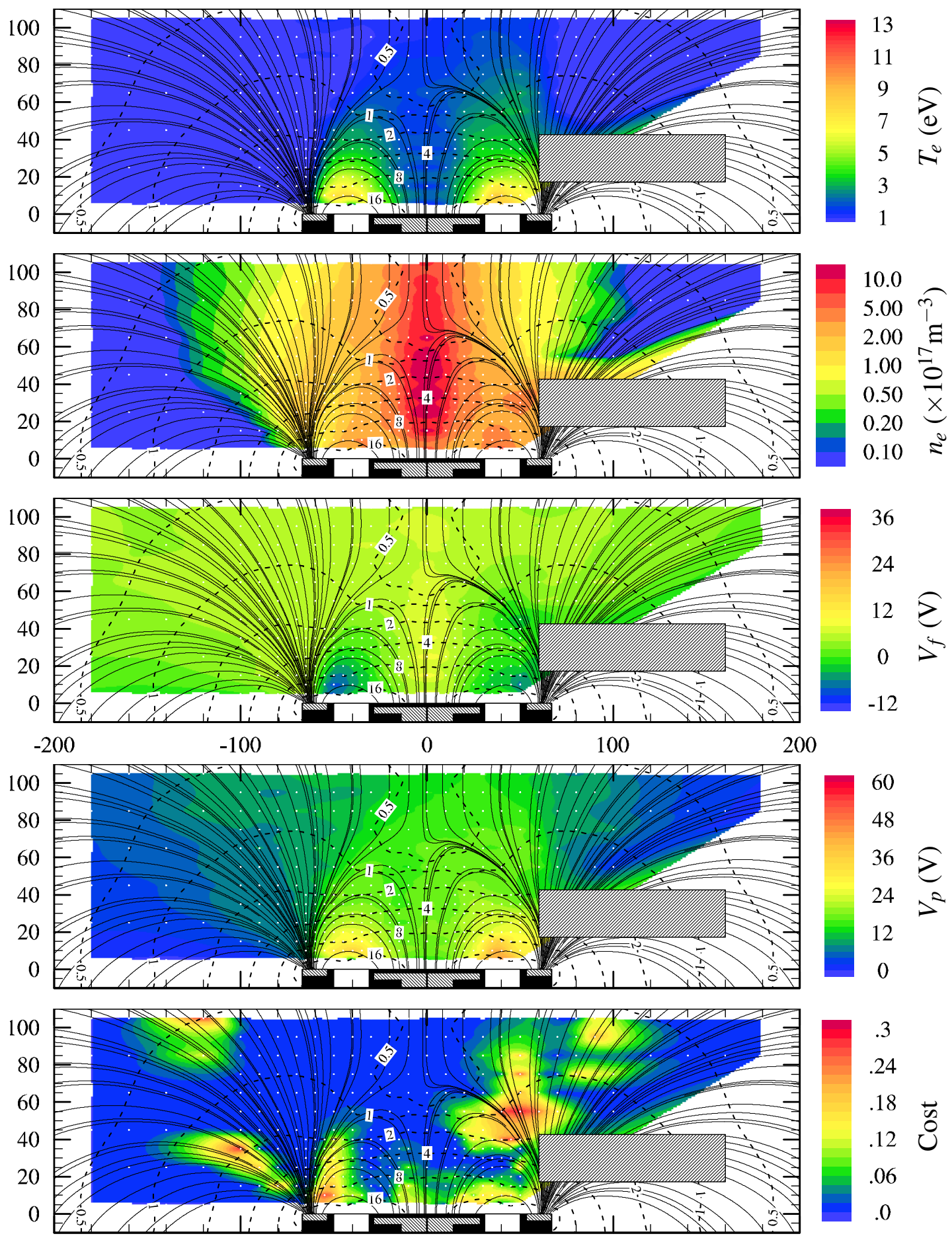

Figure C.12: Plasma properties for $\mathrm{Kr}$ with the OOP with $\dot{m}_{c}=10 \mathrm{SCCM}, r=60 \mathrm{~mm}$ 

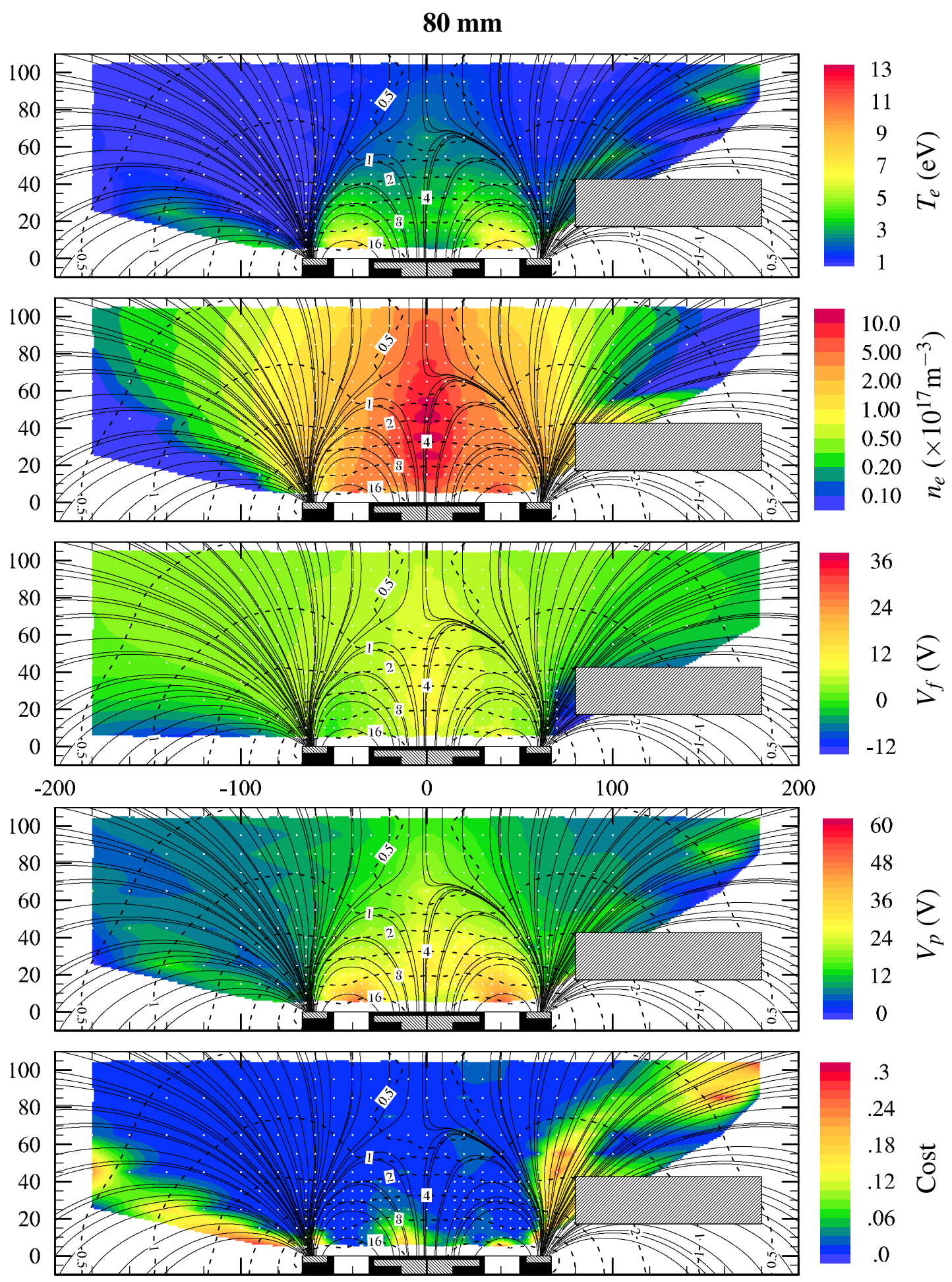

Figure C.13: Plasma properties for Kr with the OOP with $\dot{m}_{c}=10 \mathrm{SCCM}, r=80 \mathrm{~mm}$ 
$100 \mathrm{~mm}$
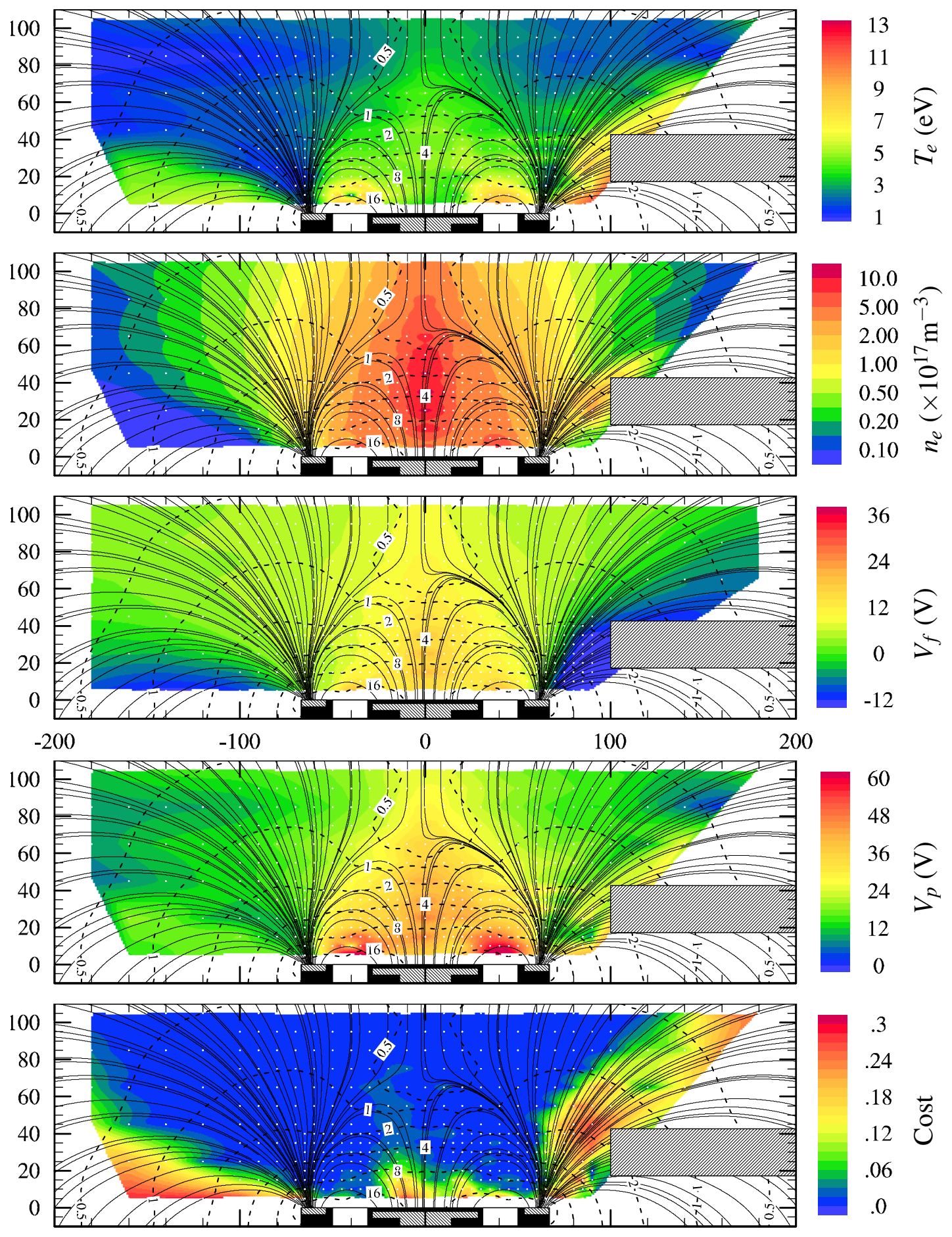

Figure C.14: Plasma properties for Kr with the OOP with $\dot{m}_{c}=10 \mathrm{SCCM}, r=100 \mathrm{~mm}$ 


\section{$120 \mathrm{~mm}$}
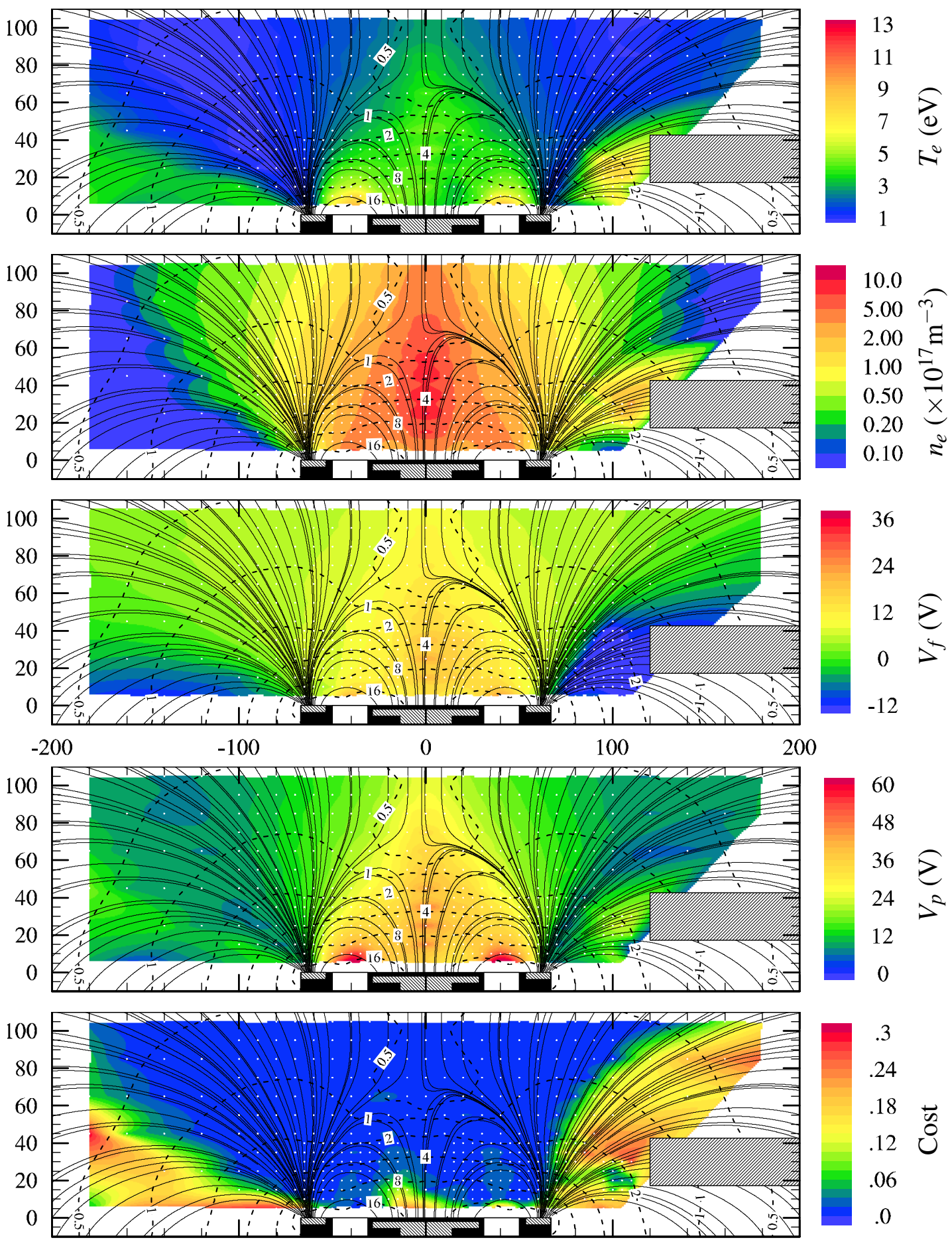

Figure C.15: Plasma properties for Kr with the OOP with $\dot{m}_{c}=10 \mathrm{SCCM}, r=120 \mathbf{m m}$ 
$200 \mathrm{~mm}$
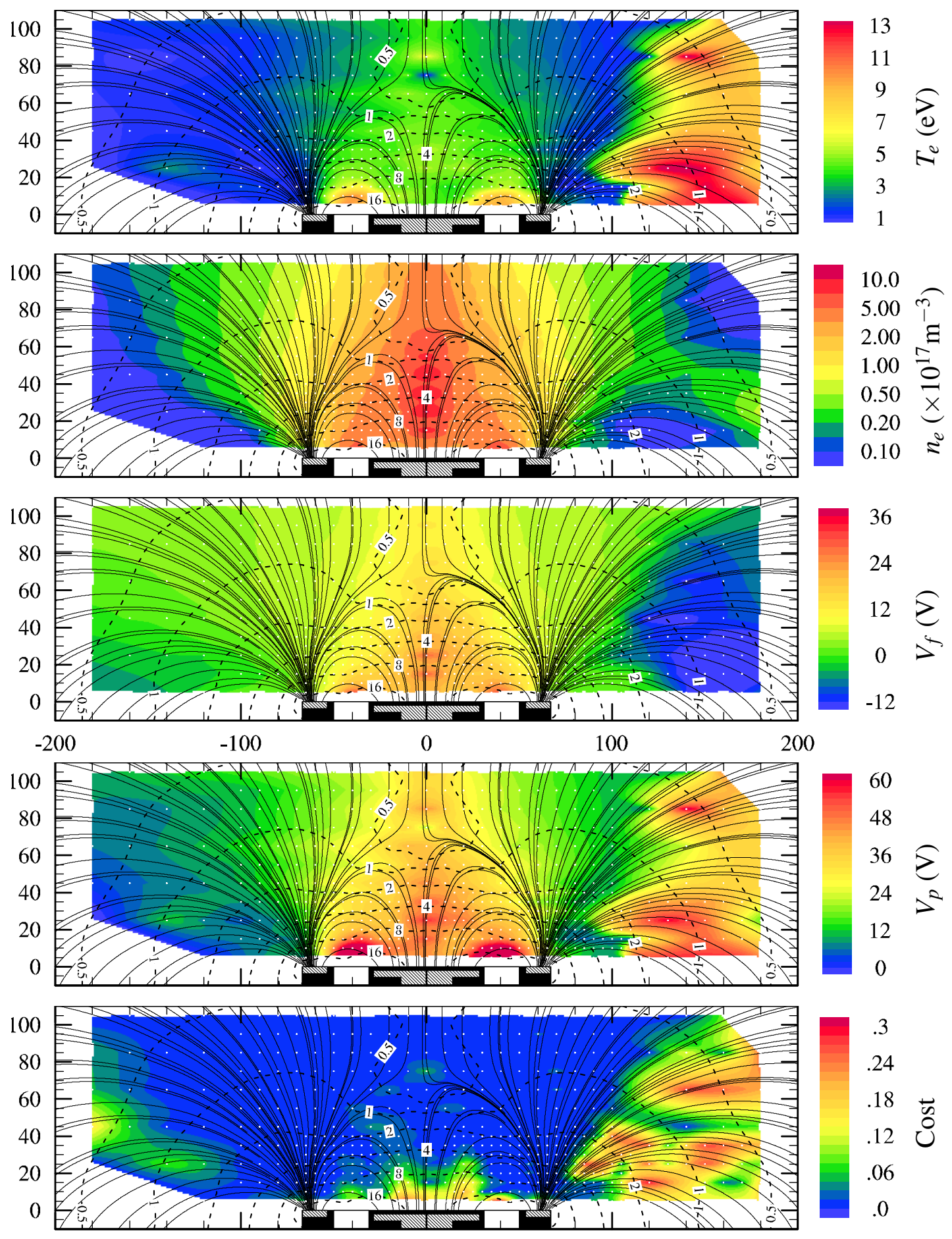

Figure C.16: Plasma properties for Kr with the OOP with $\dot{m}_{c}=10 \mathrm{SCCM}, r=200 \mathrm{~mm}$ 


\section{C.2 Kr EOP}

These data were acquired while operating the HET at $V_{d}=250 \mathrm{~V}$ with krypton and the extended outer pole. $I_{\text {mag }}$ was $1.5 \mathrm{~A}$. 

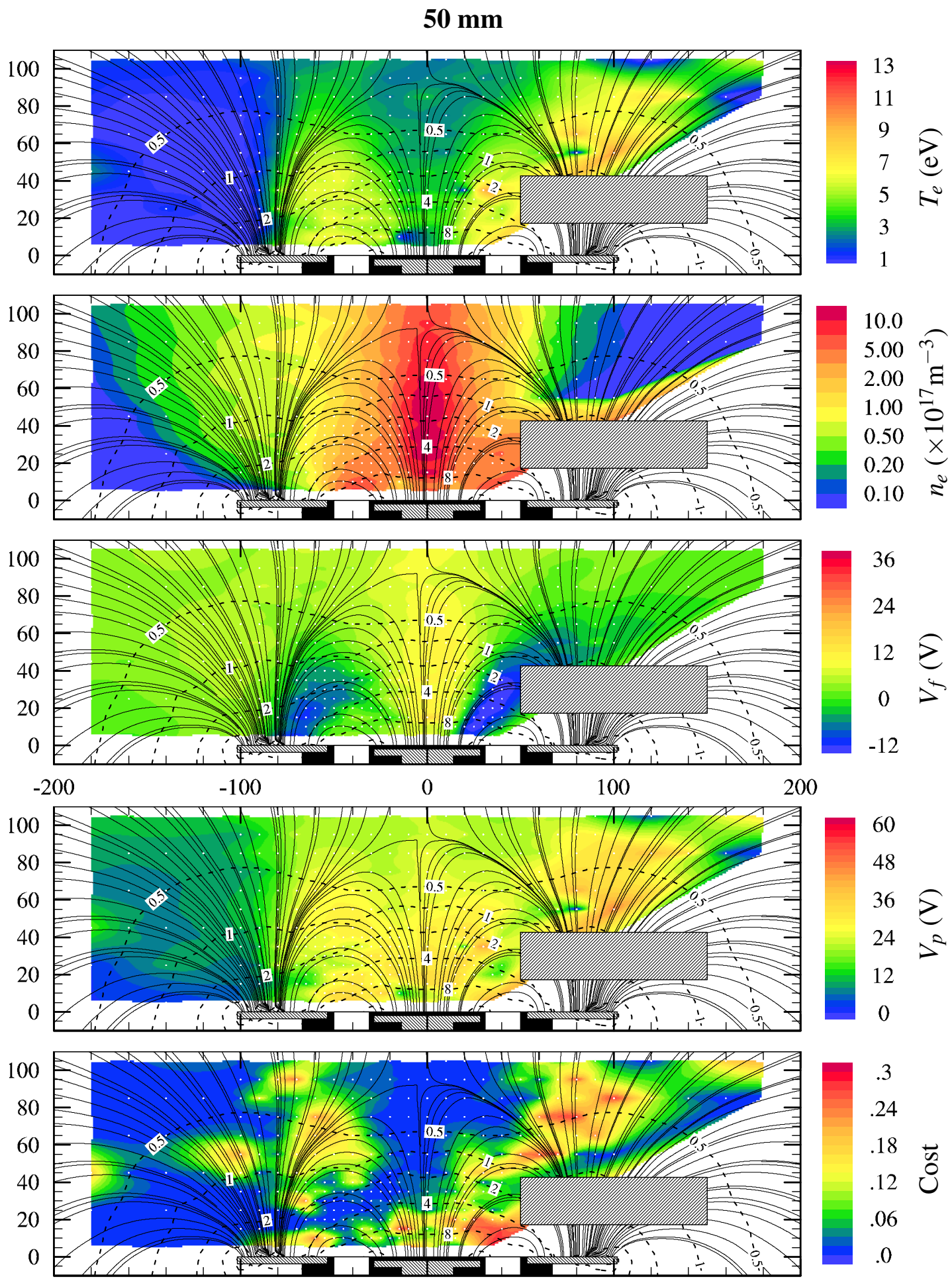

Figure C.17: Plasma properties for $\mathrm{Kr}$ with the EOP with $\dot{m}_{c}=2 \mathrm{SCCM}, r=50 \mathrm{~mm}$ 
$60 \mathrm{~mm}$
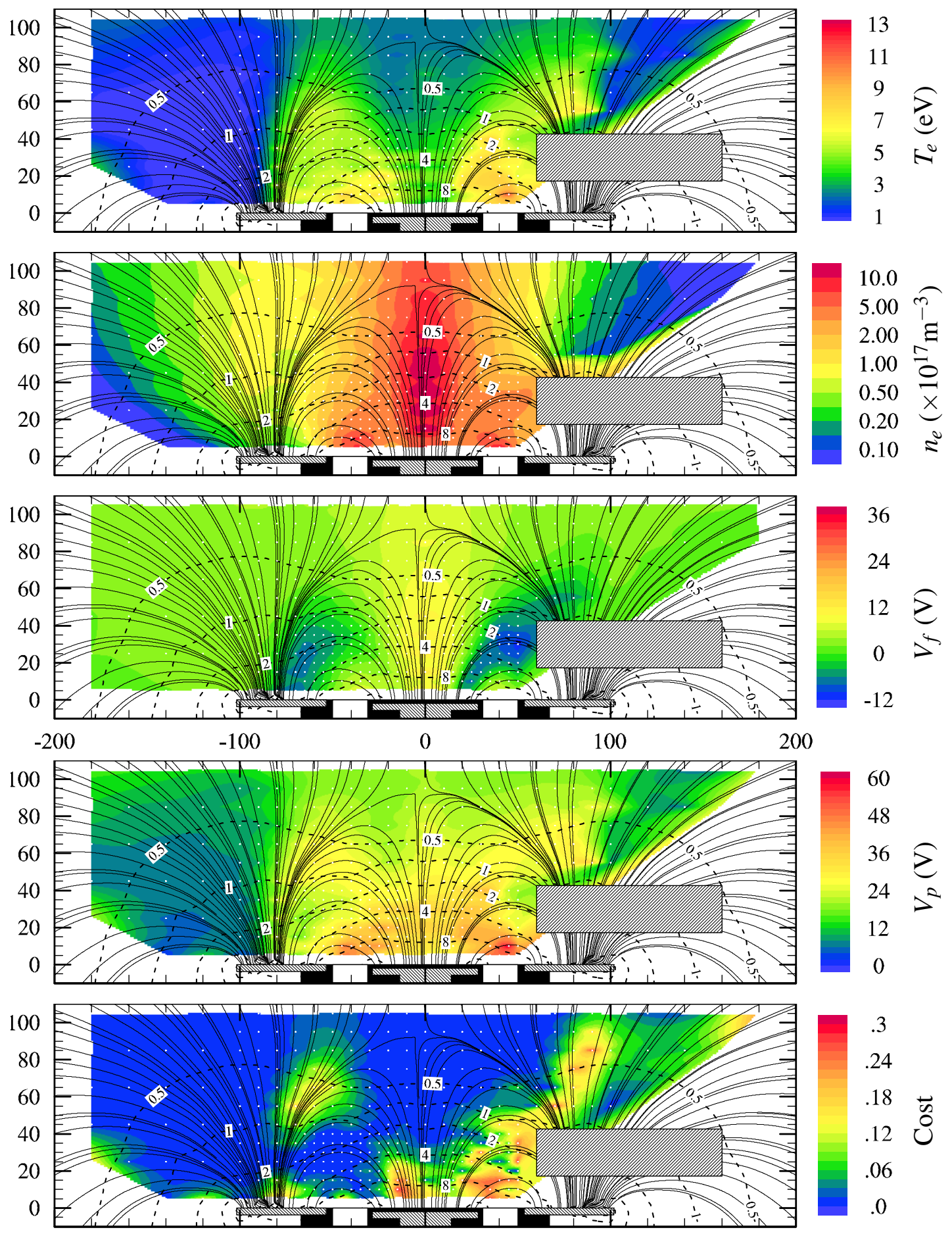

Figure C.18: Plasma properties for Kr with the EOP with $\dot{m}_{c}=2 \mathrm{SCCM}, r=60 \mathrm{~mm}$ 
$80 \mathrm{~mm}$
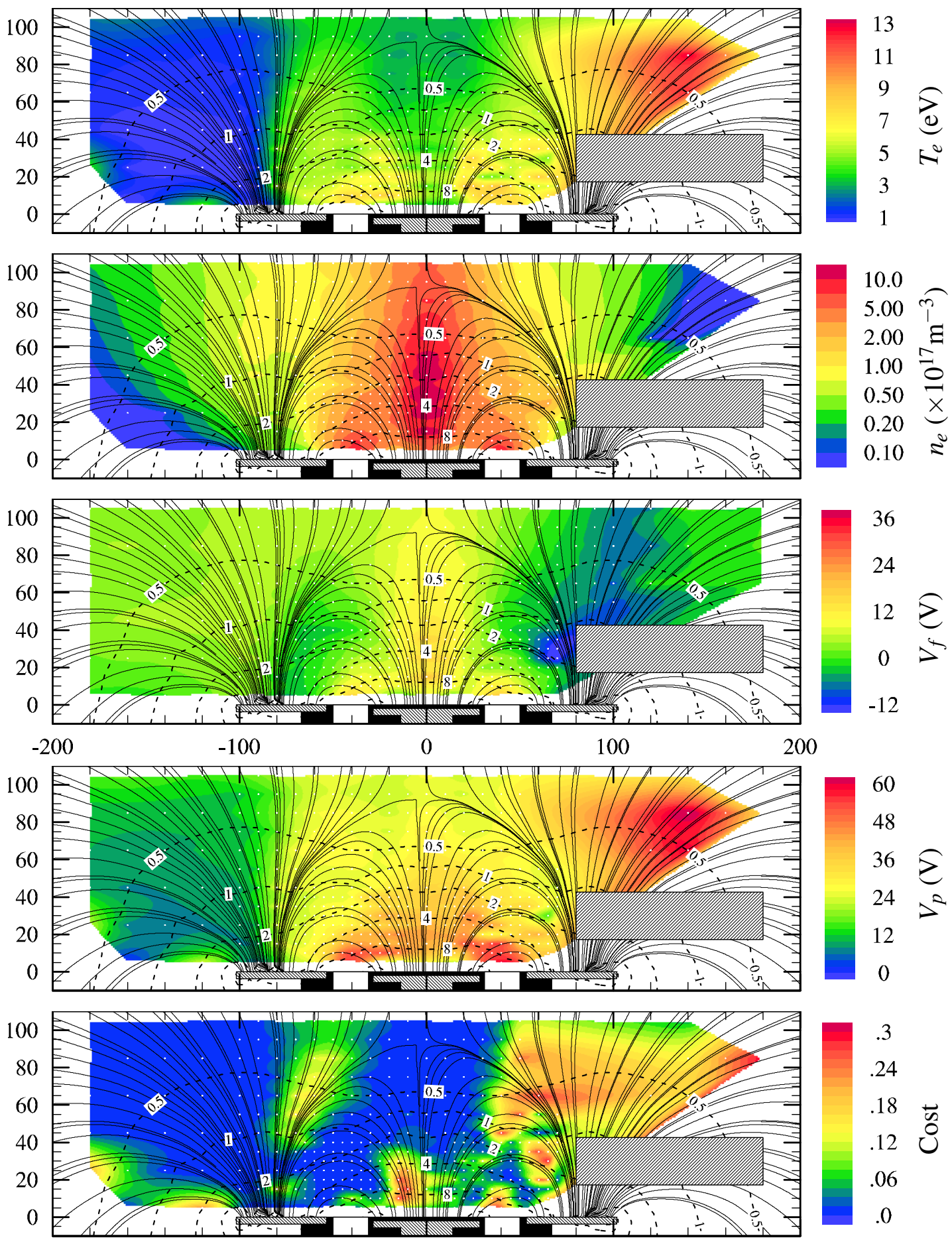

Figure C.19: Plasma properties for $\mathrm{Kr}$ with the EOP with $\dot{m}_{c}=2 \mathrm{SCCM}, r=80 \mathrm{~mm}$ 


\section{$100 \mathrm{~mm}$}
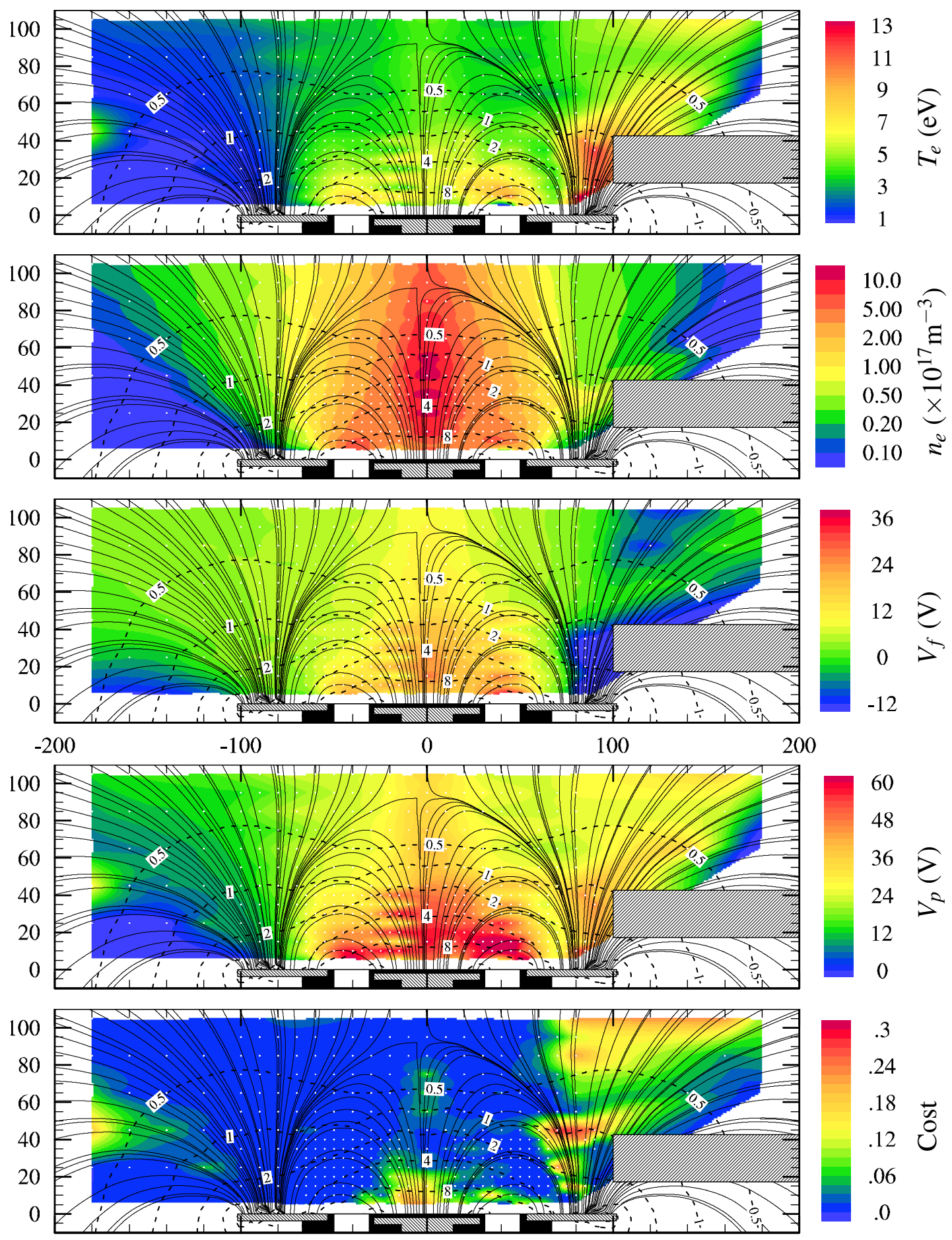

Figure C.20: Plasma properties for Kr with the EOP with $\dot{m}_{c}=2 \mathrm{SCCM}, r=100 \mathrm{~mm}$ 


\section{$120 \mathrm{~mm}$}
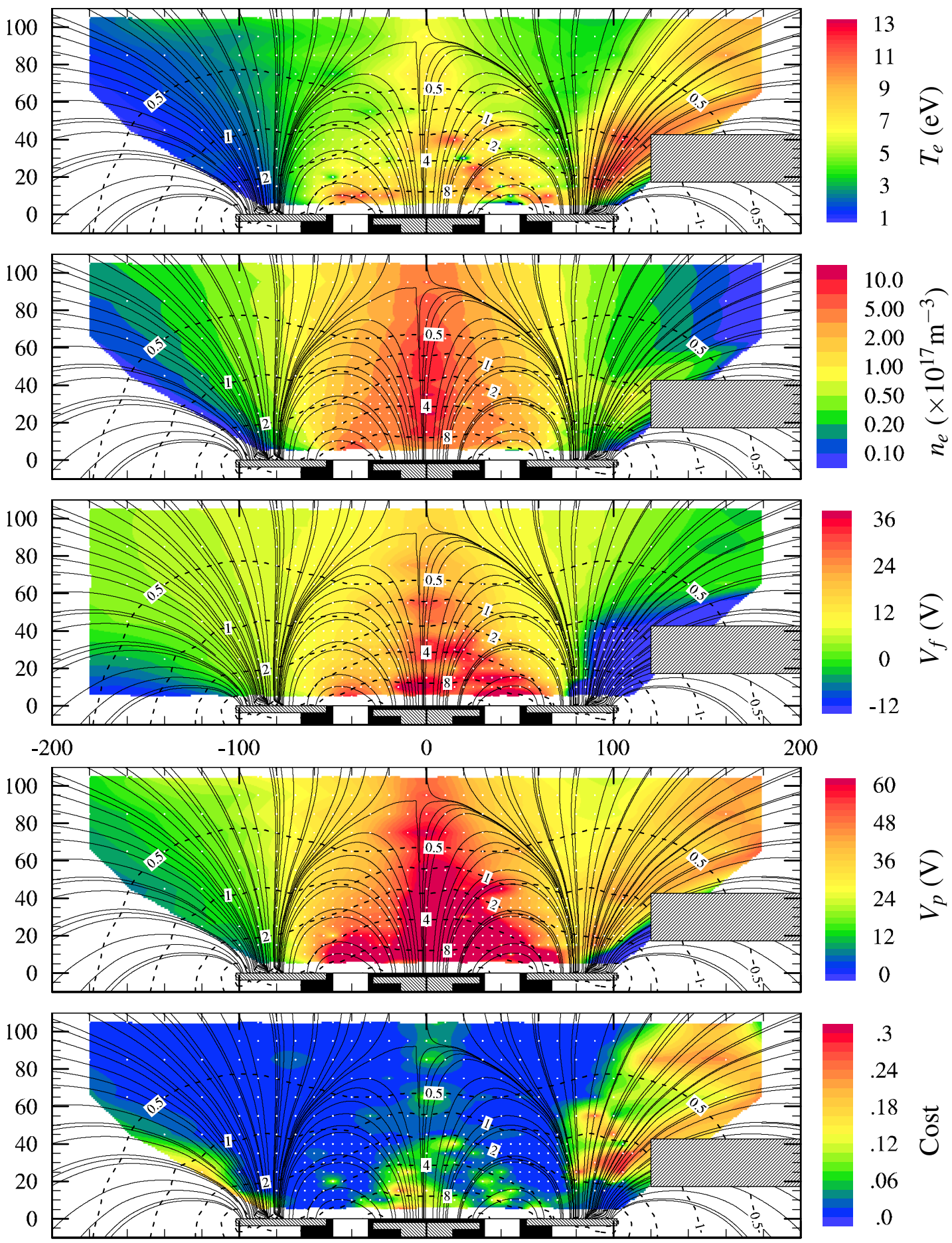

Figure C.21: Plasma properties for Kr with the EOP with $\dot{m}_{c}=2 \mathrm{SCCM}, r=120 \mathrm{~mm}$ 
$50 \mathrm{~mm}$
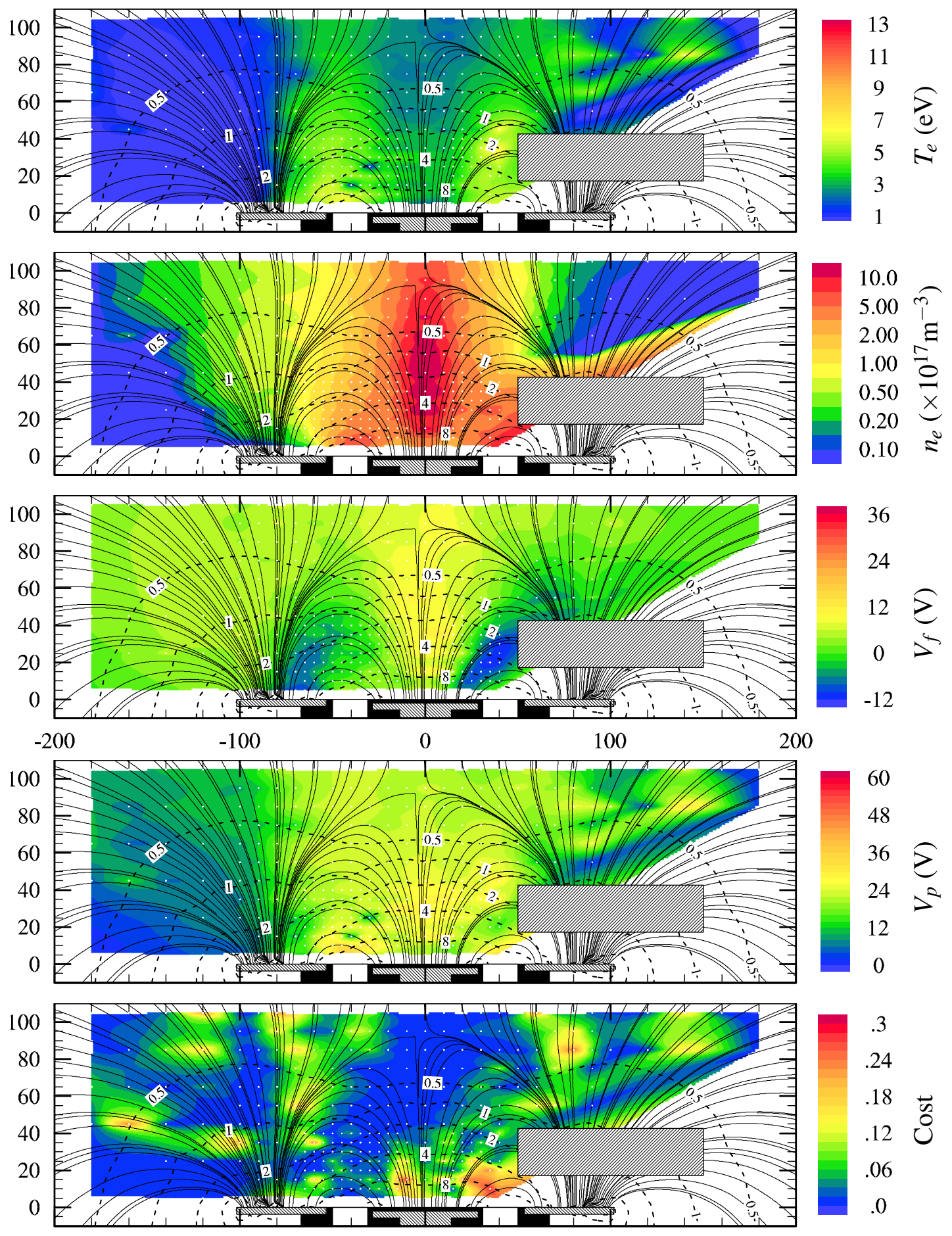

Figure C.22: Plasma properties for $\mathrm{Kr}$ with the EOP with $\dot{m}_{c}=5 \mathrm{SCCM}, r=50 \mathrm{~mm}$ 

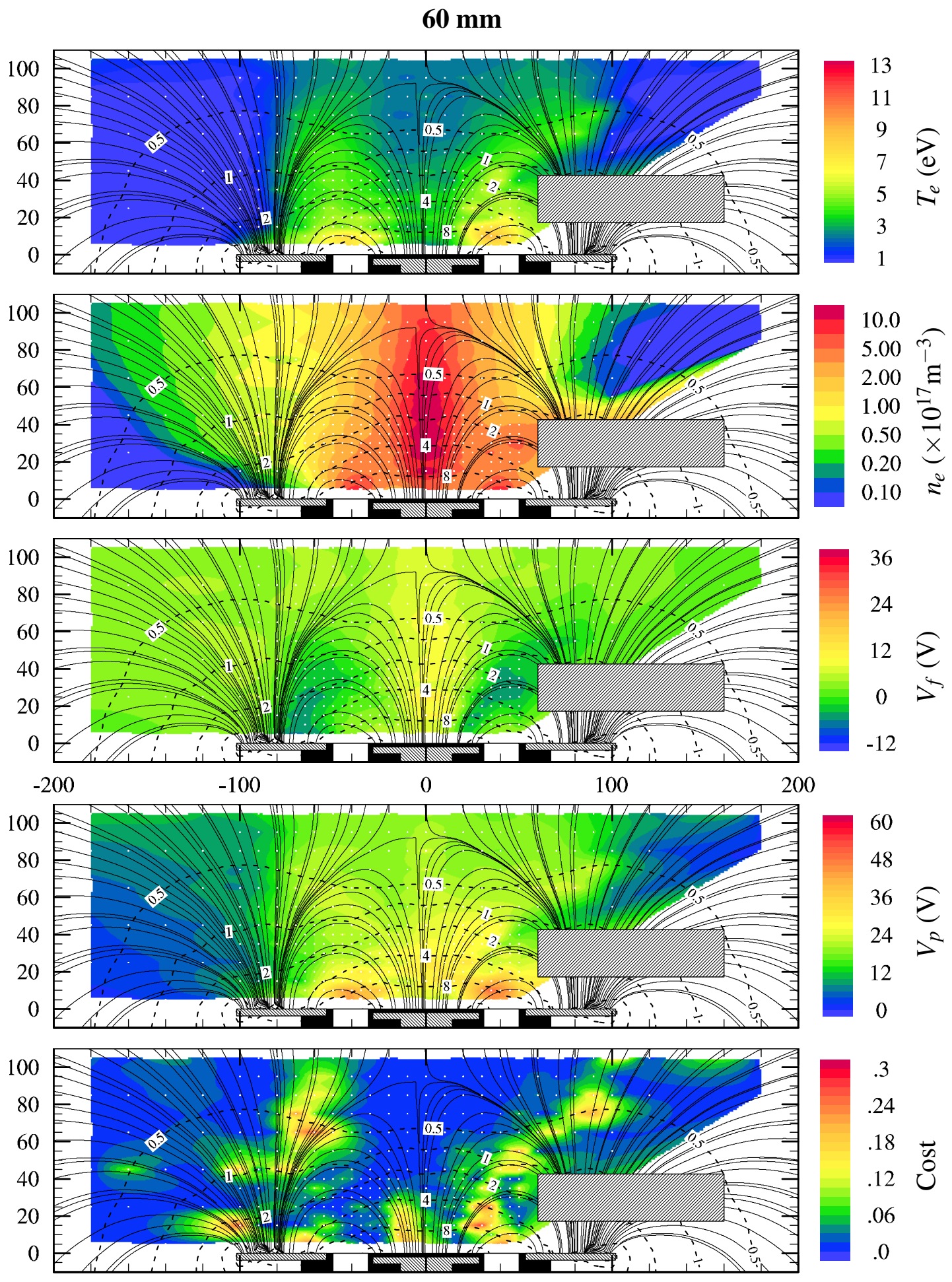

Figure C.23: Plasma properties for Kr with the EOP with $\dot{m}_{c}=5 \mathrm{SCCM}, r=60 \mathrm{~mm}$ 
$80 \mathrm{~mm}$
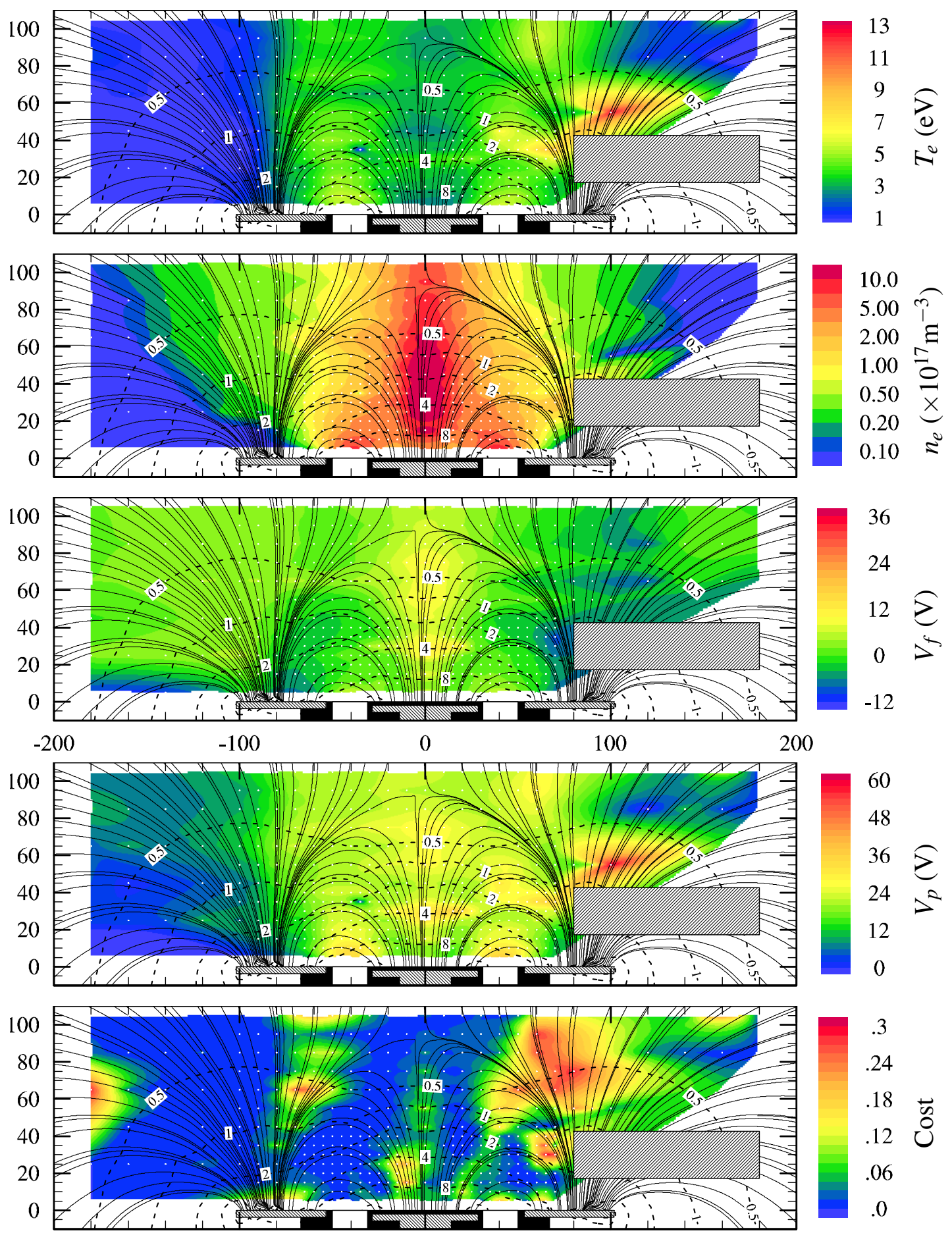

Figure C.24: Plasma properties for $\mathrm{Kr}$ with the EOP with $\dot{m}_{c}=5 \mathrm{SCCM}, r=80 \mathrm{~mm}$ 


\section{$100 \mathrm{~mm}$}
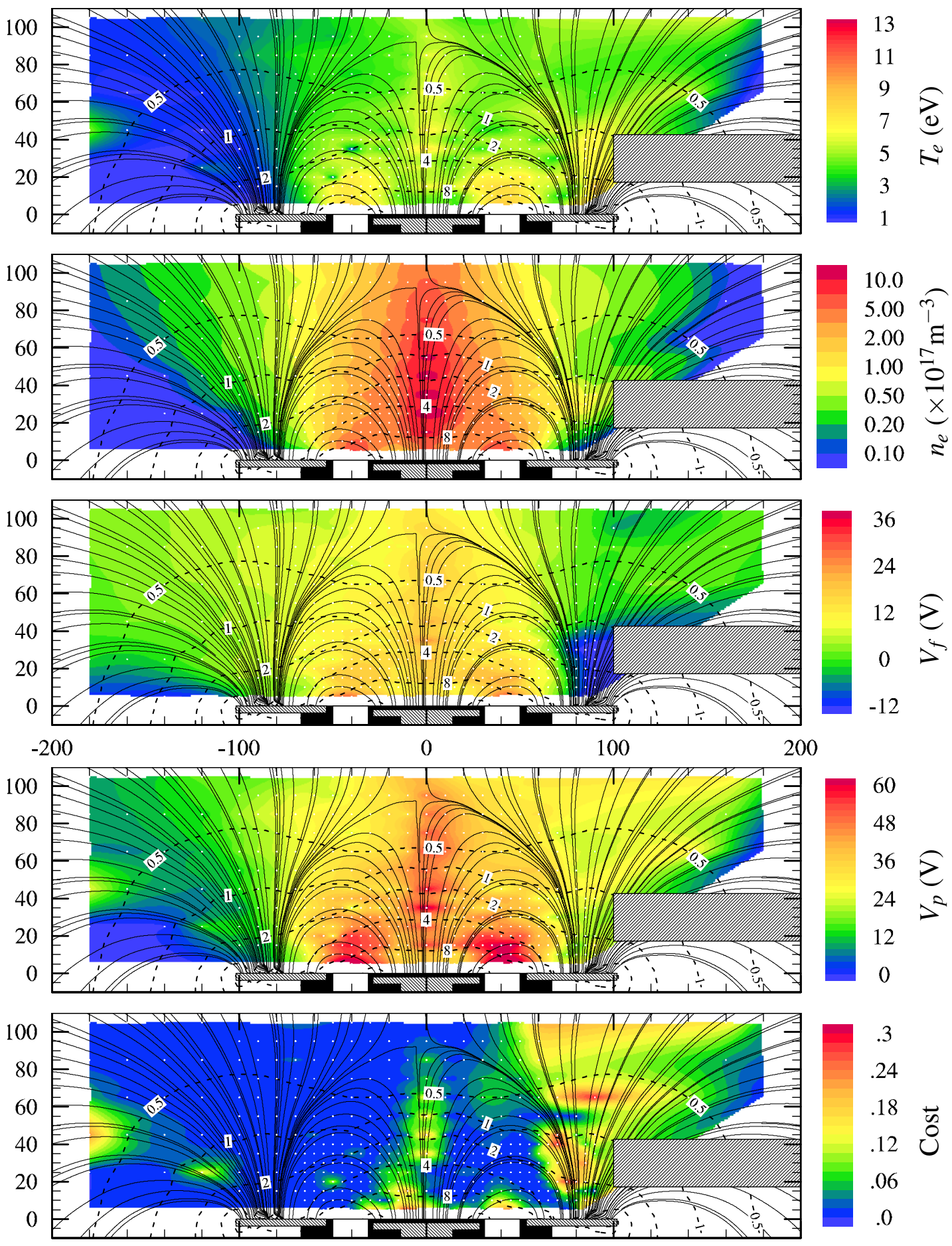

Figure C.25: Plasma properties for Kr with the EOP with $\dot{m}_{c}=5 \mathrm{SCCM}, r=100 \mathrm{~mm}$ 


\section{$120 \mathrm{~mm}$}
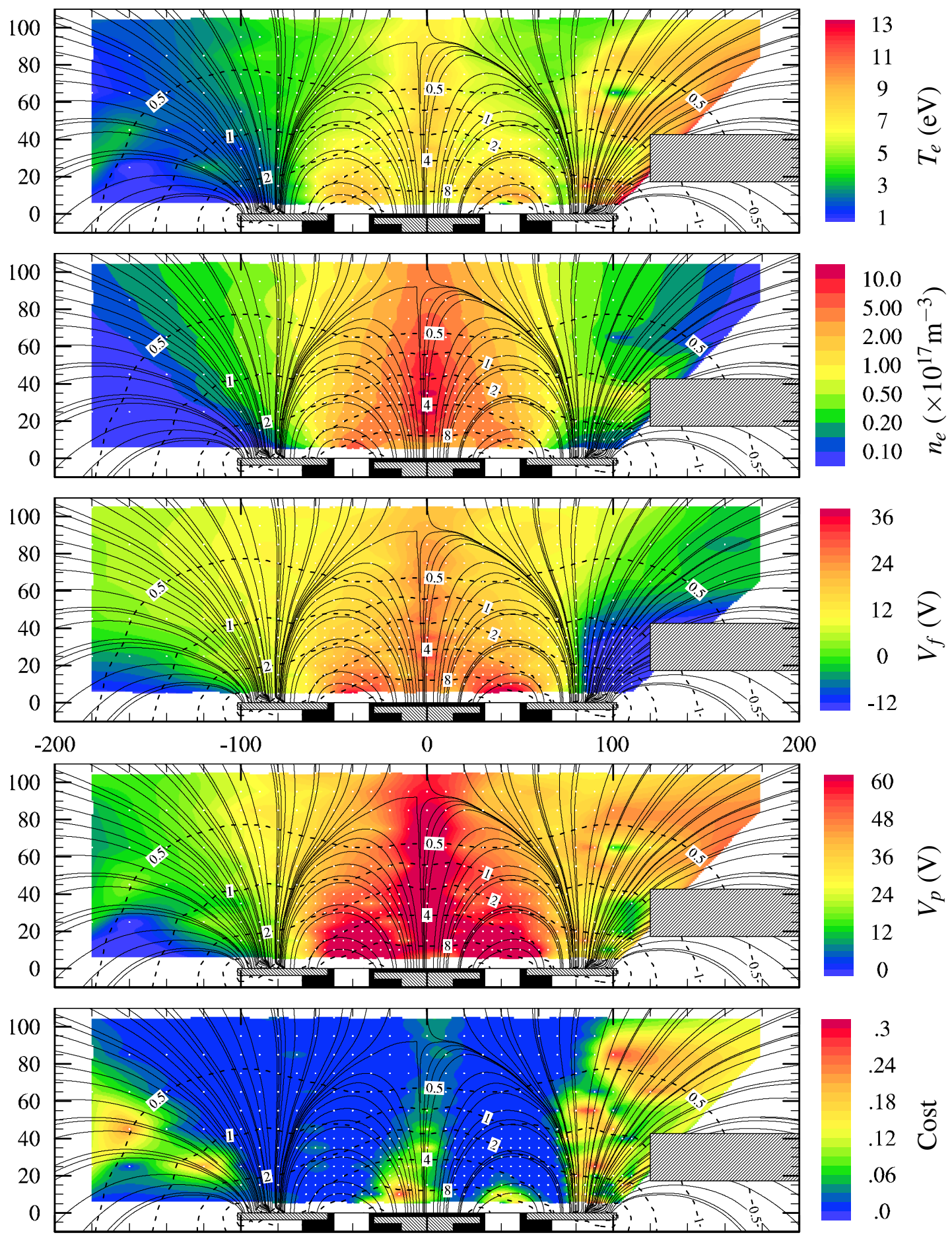

Figure C.26: Plasma properties for Kr with the EOP with $\dot{m}_{c}=5 \mathrm{SCCM}, r=120 \mathrm{~mm}$ 


\section{$200 \mathrm{~mm}$}
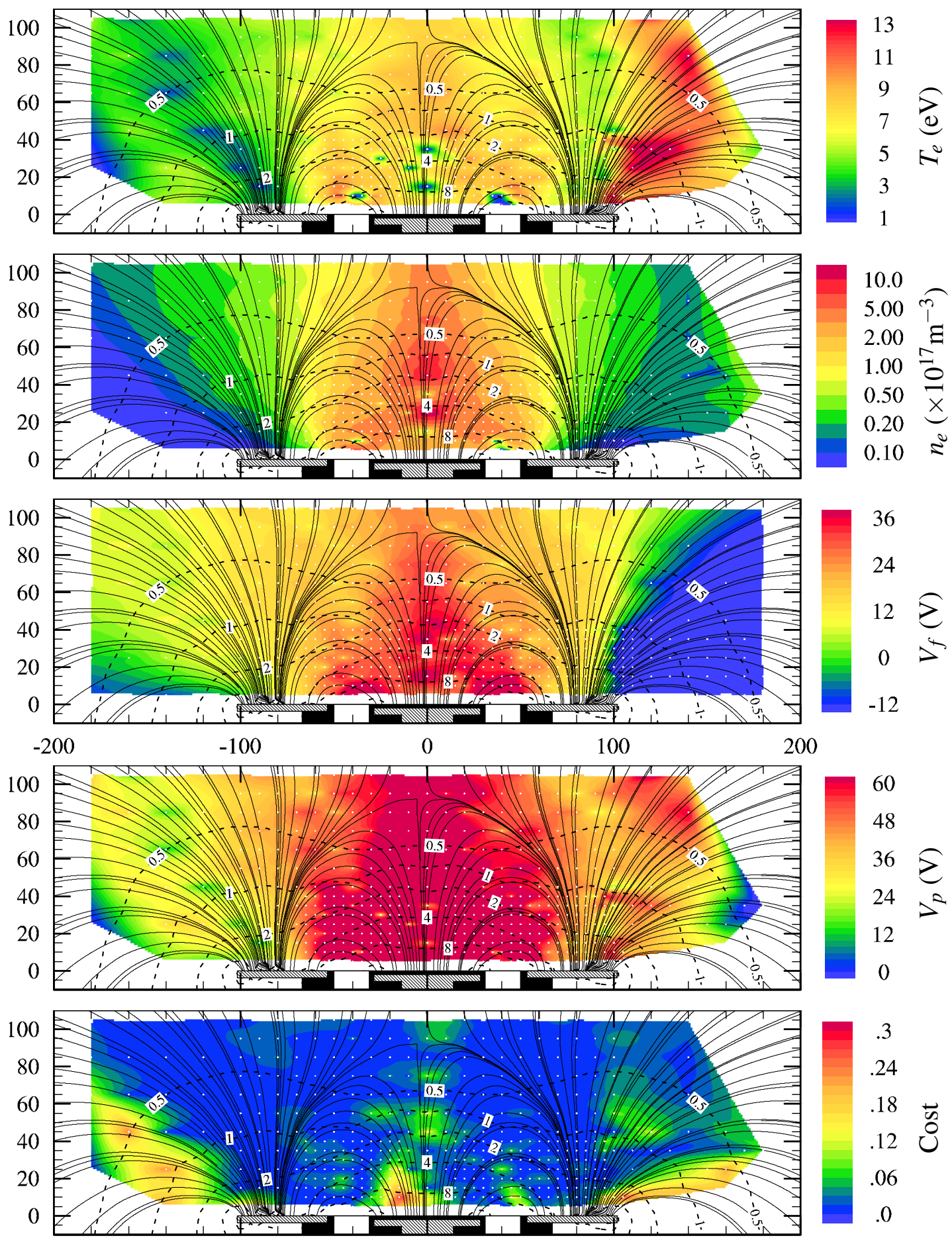

Figure C.27: Plasma properties for Kr with the EOP with $\dot{m}_{c}=5 \mathrm{SCCM}, r=200 \mathrm{~mm}$ 
$50 \mathrm{~mm}$
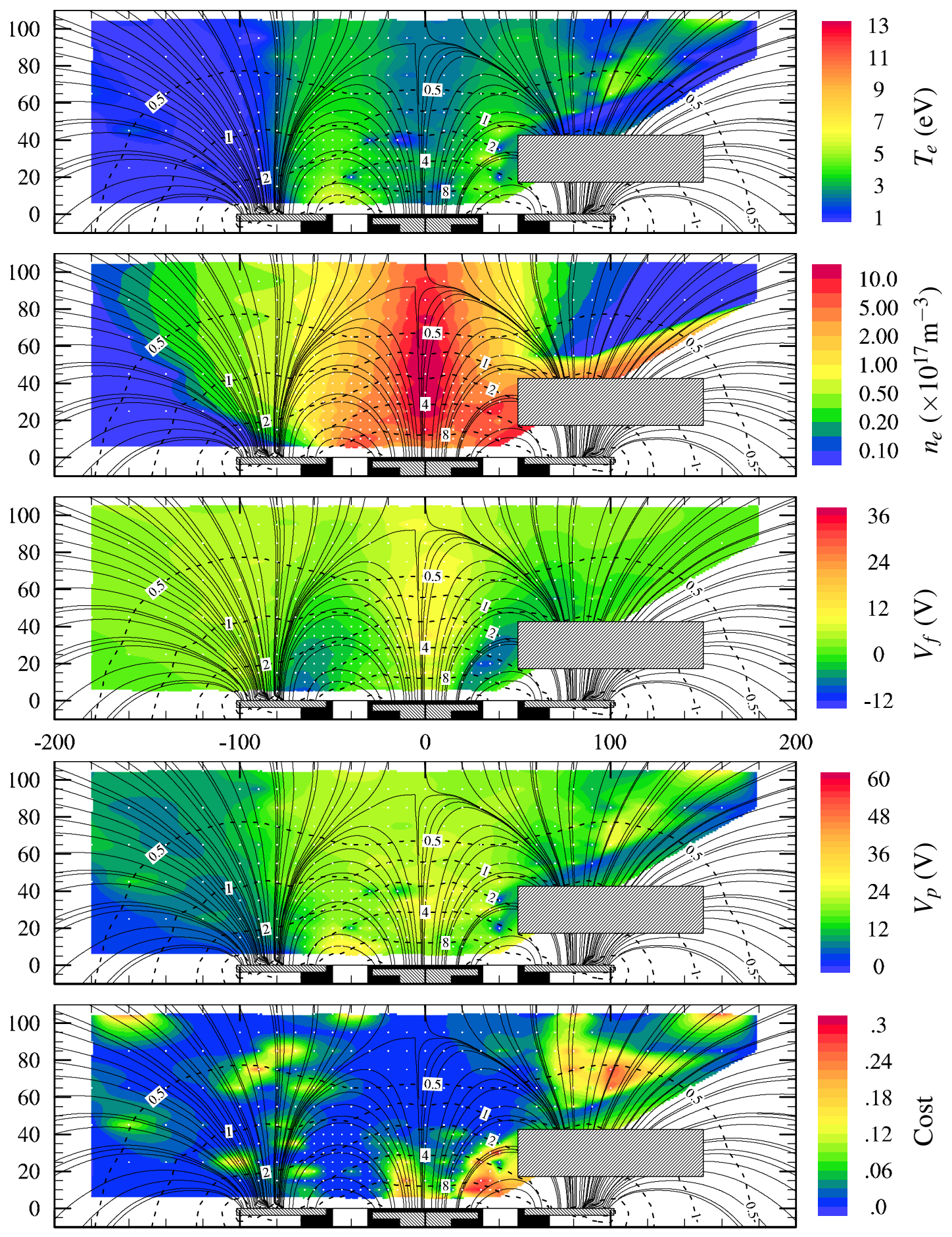

Figure C.28: Plasma properties for Kr with the EOP with $\dot{m}_{c}=10 \mathrm{SCCM}, r=50 \mathrm{~mm}$ 

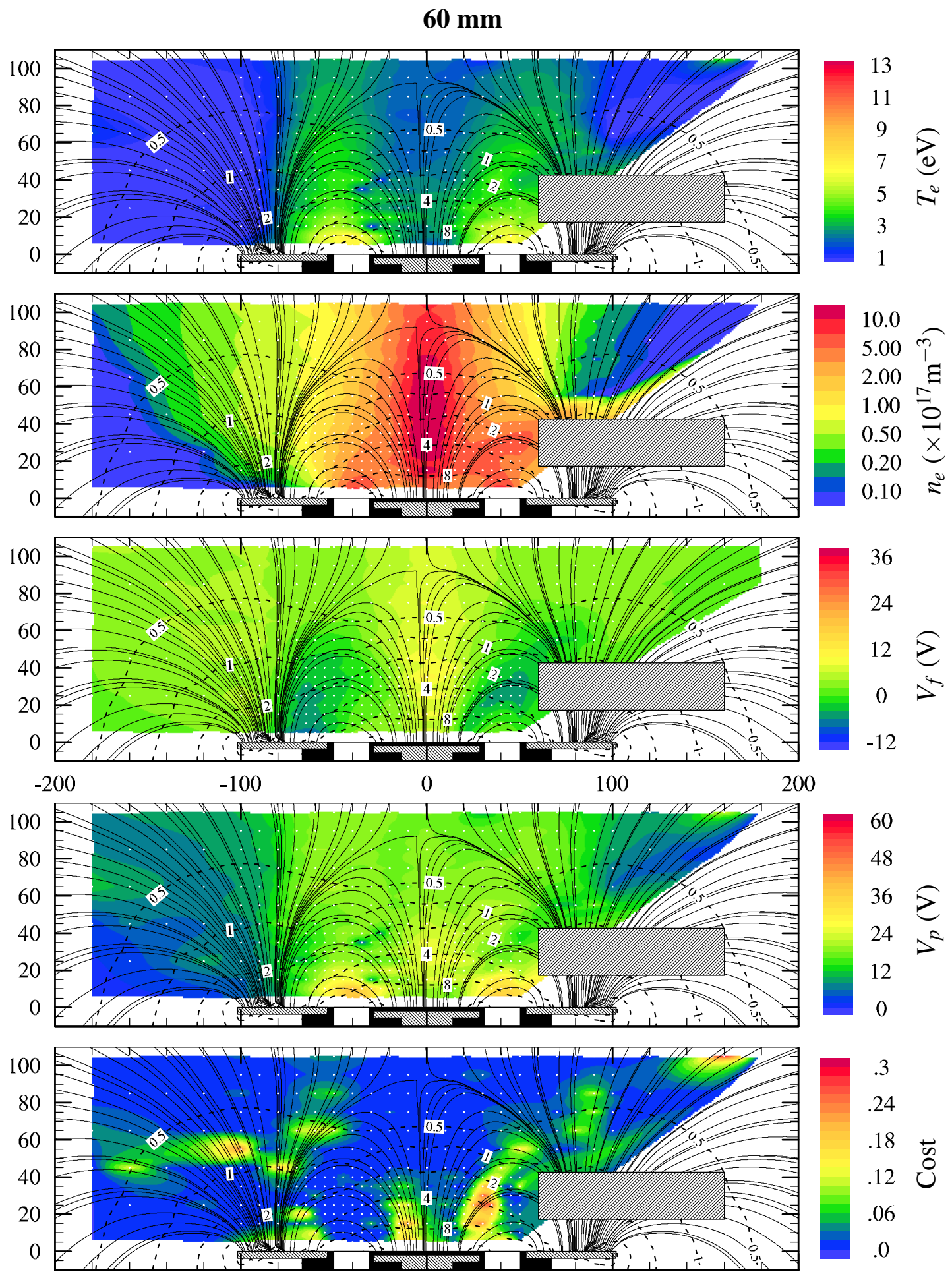

Figure C.29: Plasma properties for Kr with the EOP with $\dot{m}_{c}=10 \mathrm{SCCM}, r=60 \mathrm{~mm}$ 
$80 \mathrm{~mm}$
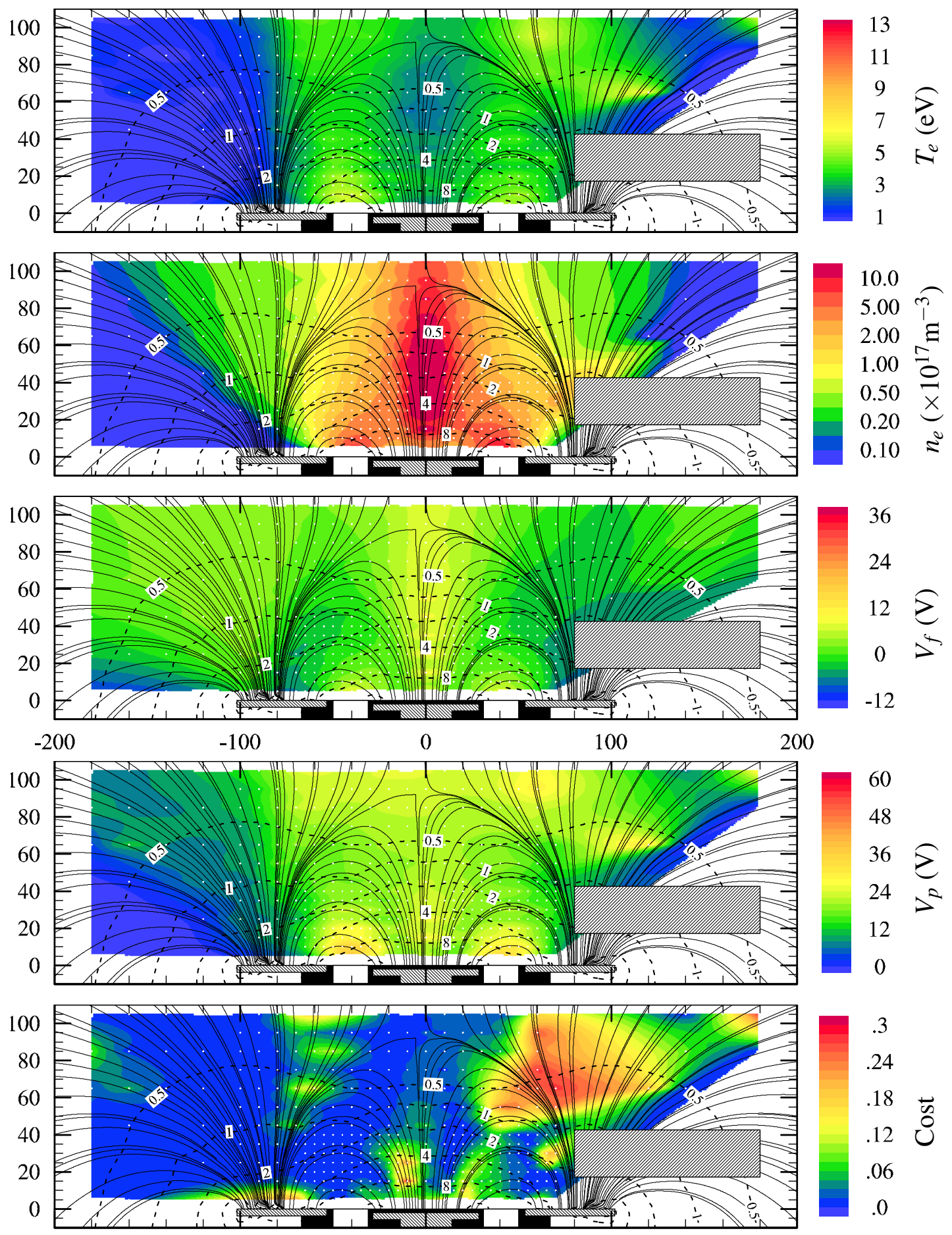

Figure C.30: Plasma properties for Kr with the EOP with $\dot{m}_{c}=10 \mathrm{SCCM}, r=80 \mathrm{~mm}$ 


\section{$100 \mathrm{~mm}$}
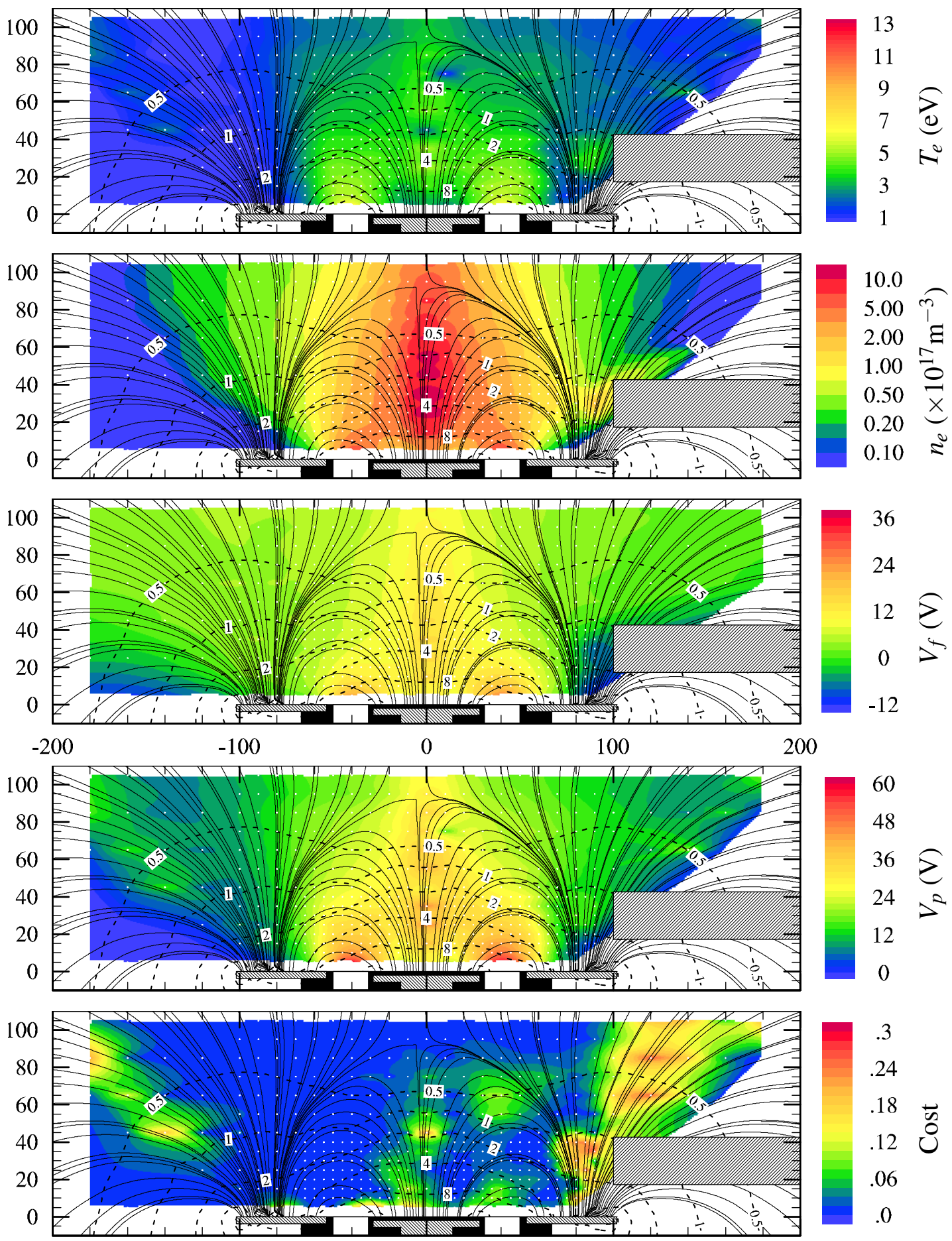

Figure C.31: Plasma properties for Kr with the EOP with $\dot{m}_{c}=10 \mathrm{SCCM}, r=100 \mathrm{~mm}$ 


\section{$120 \mathrm{~mm}$}
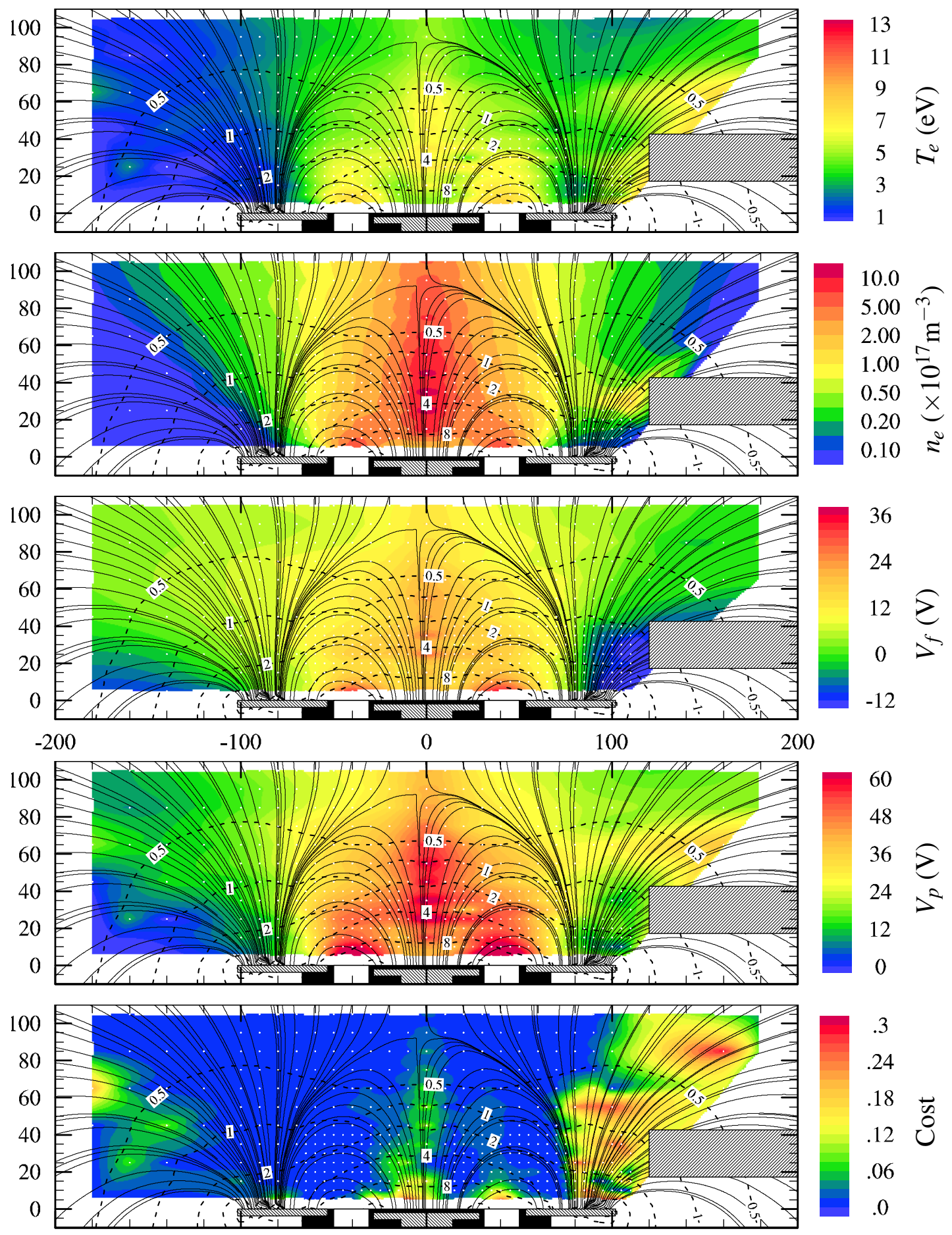

Figure C.32: Plasma properties for Kr with the EOP with $\dot{m}_{c}=10 \mathrm{SCCM}, r=120 \mathbf{m m}$ 


\section{$200 \mathrm{~mm}$}
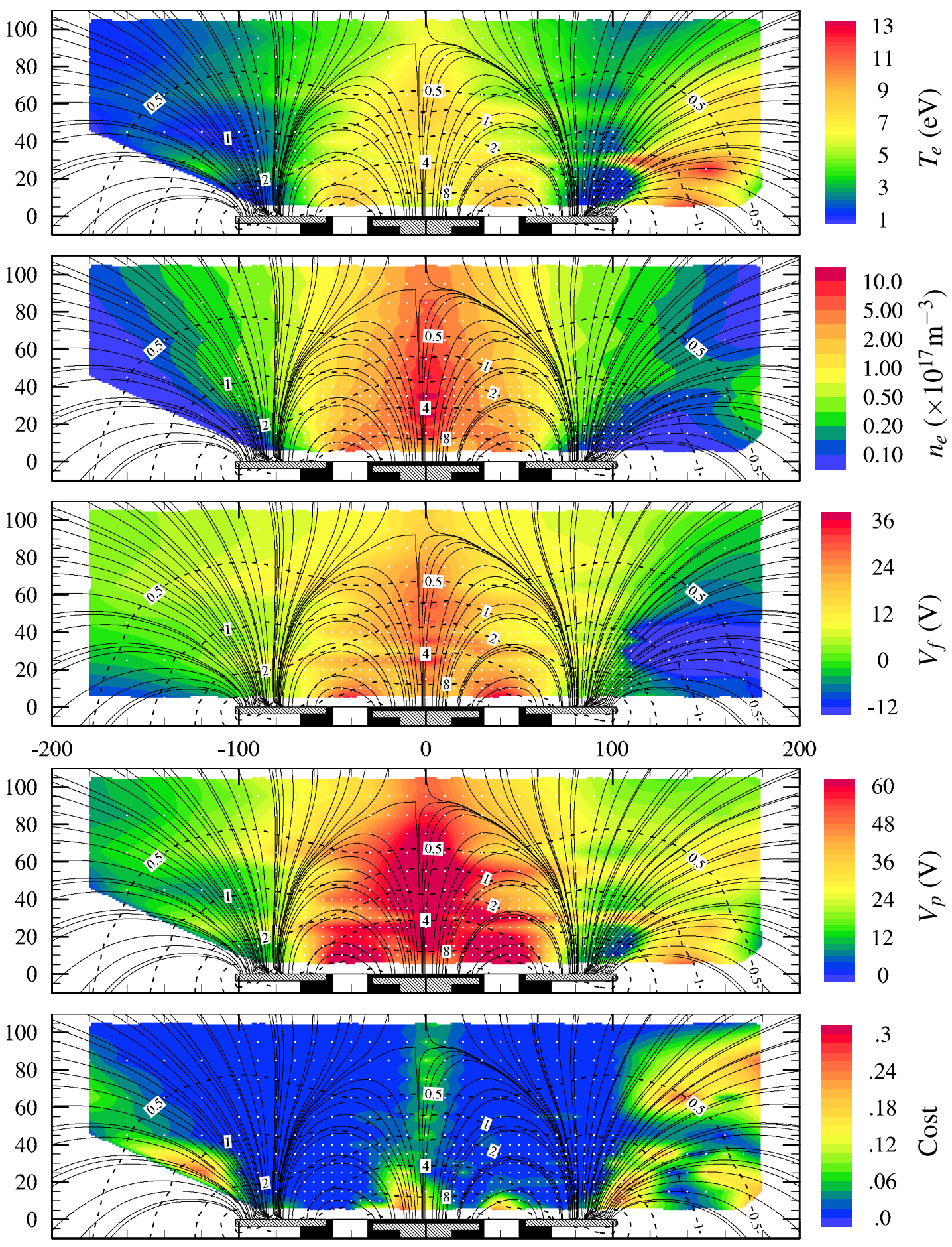

Figure C.33: Plasma properties for Kr with the EOP with $\dot{m}_{c}=10$ SCCM, $r=200 \mathrm{~mm}$ 


\section{C.3 Xe}

These data were acquired while operating the HET at $V_{d}=250 \mathrm{~V}$ with xenon and the original outer pole. $I_{\mathrm{mag}}$ was $4 \mathrm{~A}$. Due to excessive probe heating, all of the measurements except for the floating potential should be considered unreliable (see Appendix B). However, data on the left half of the figure, where the probe had not yet entered the hot regions of the plasma, is slightly more believable. The floating potential data is considered reliable as the probe heating had little effect on this measurement. 
$60 \mathrm{~mm}$
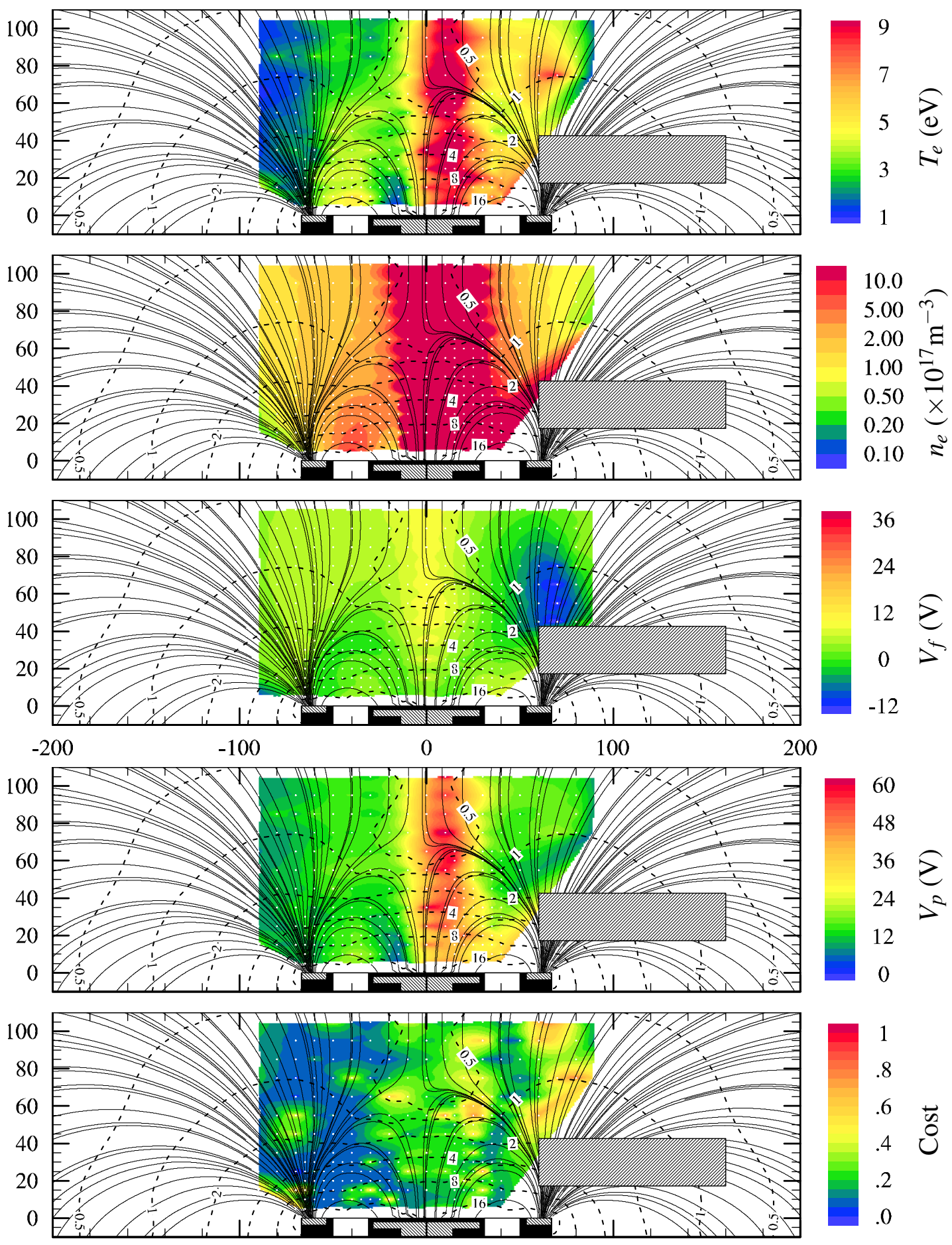

Figure C.34: Plasma properties for Xe with $\dot{m}_{c}=2 \mathrm{SCCM}, r=60 \mathrm{~mm}$ 
$80 \mathrm{~mm}$
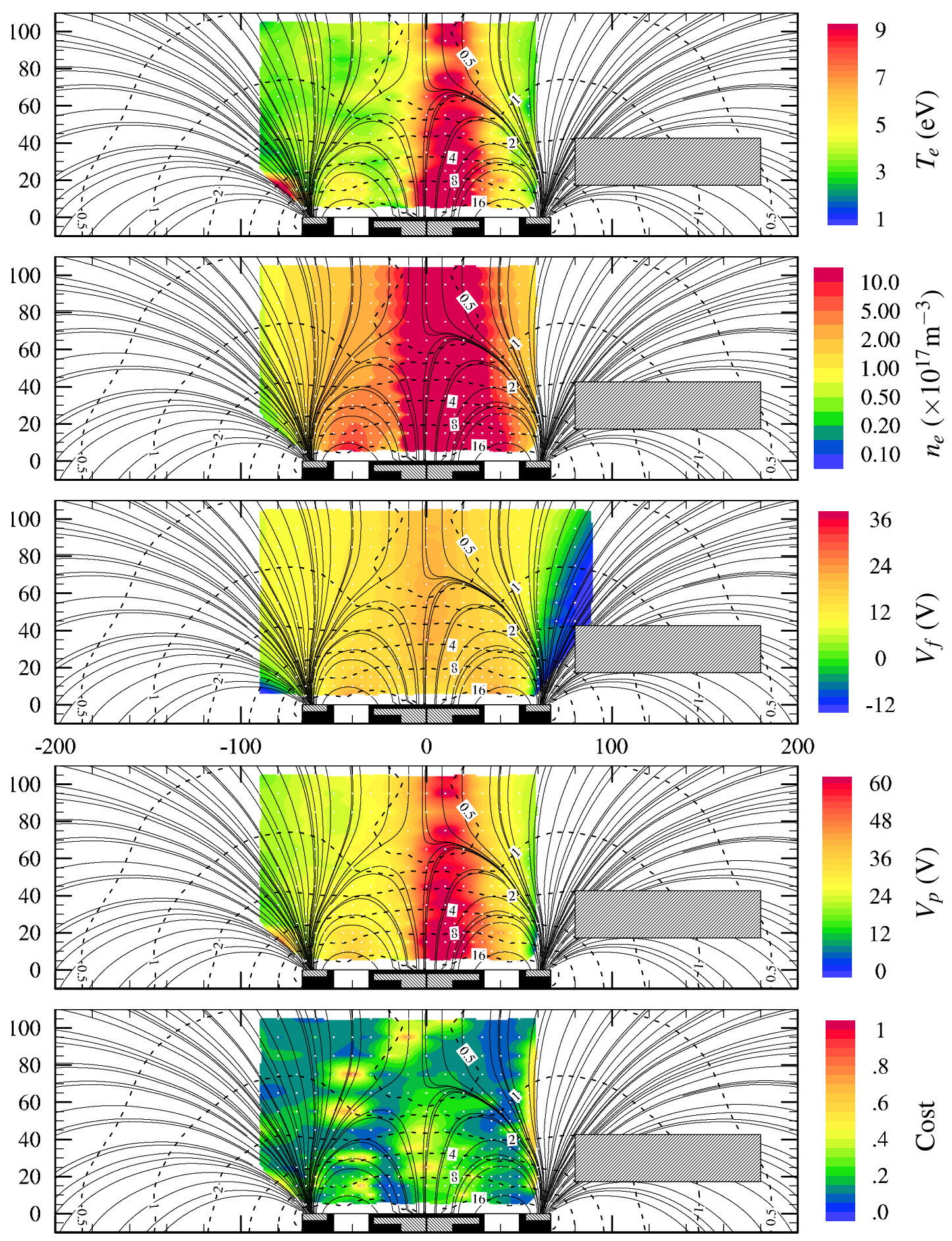

Figure C.35: Plasma properties for Xe with $\dot{m}_{c}=2$ SCCM, $r=80 \mathrm{~mm}$ 
$120 \mathrm{~mm}$
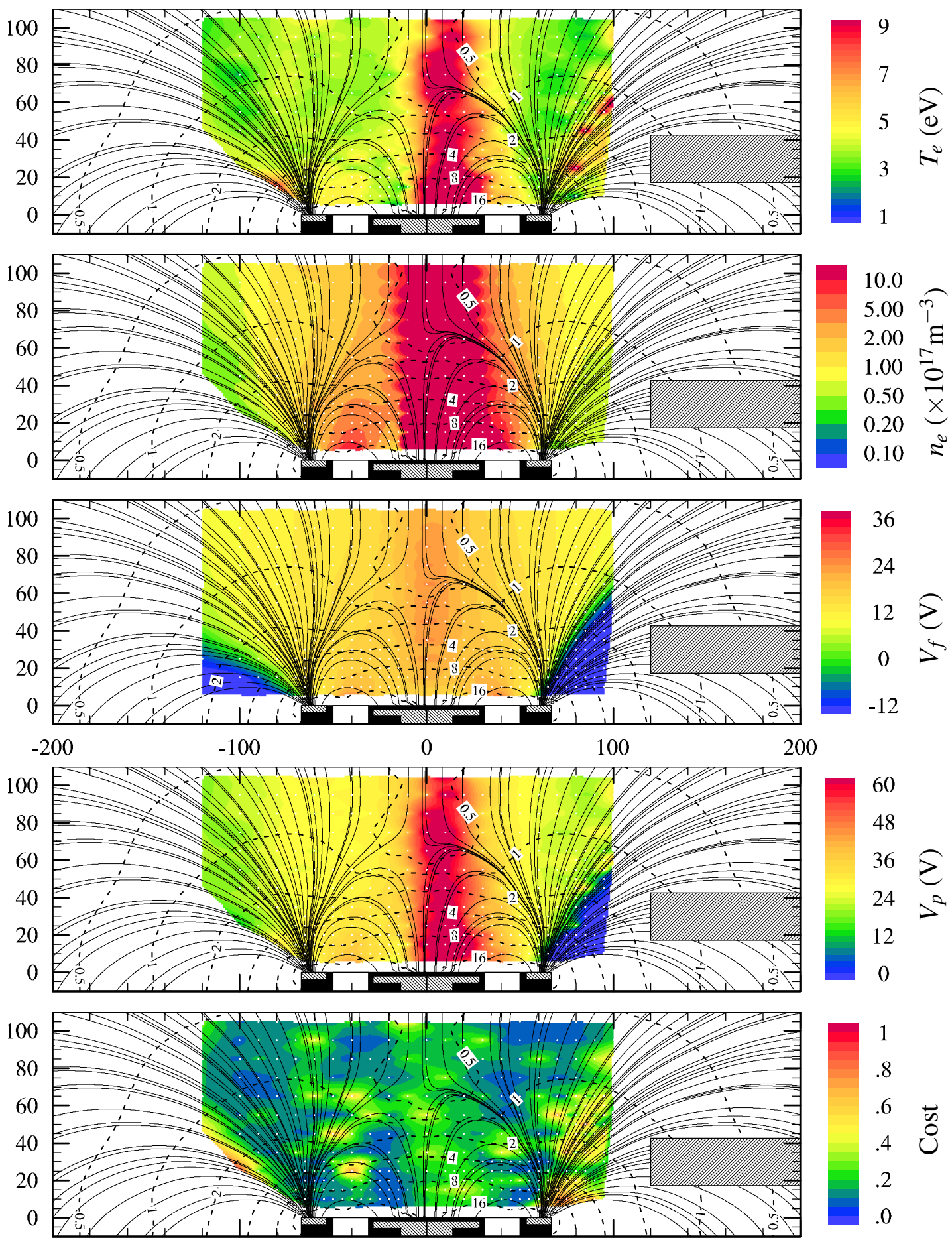

Figure C.36: Plasma properties for Xe with $\dot{m}_{c}=2$ SCCM, $r=120 \mathrm{~mm}$ 
$60 \mathrm{~mm}$
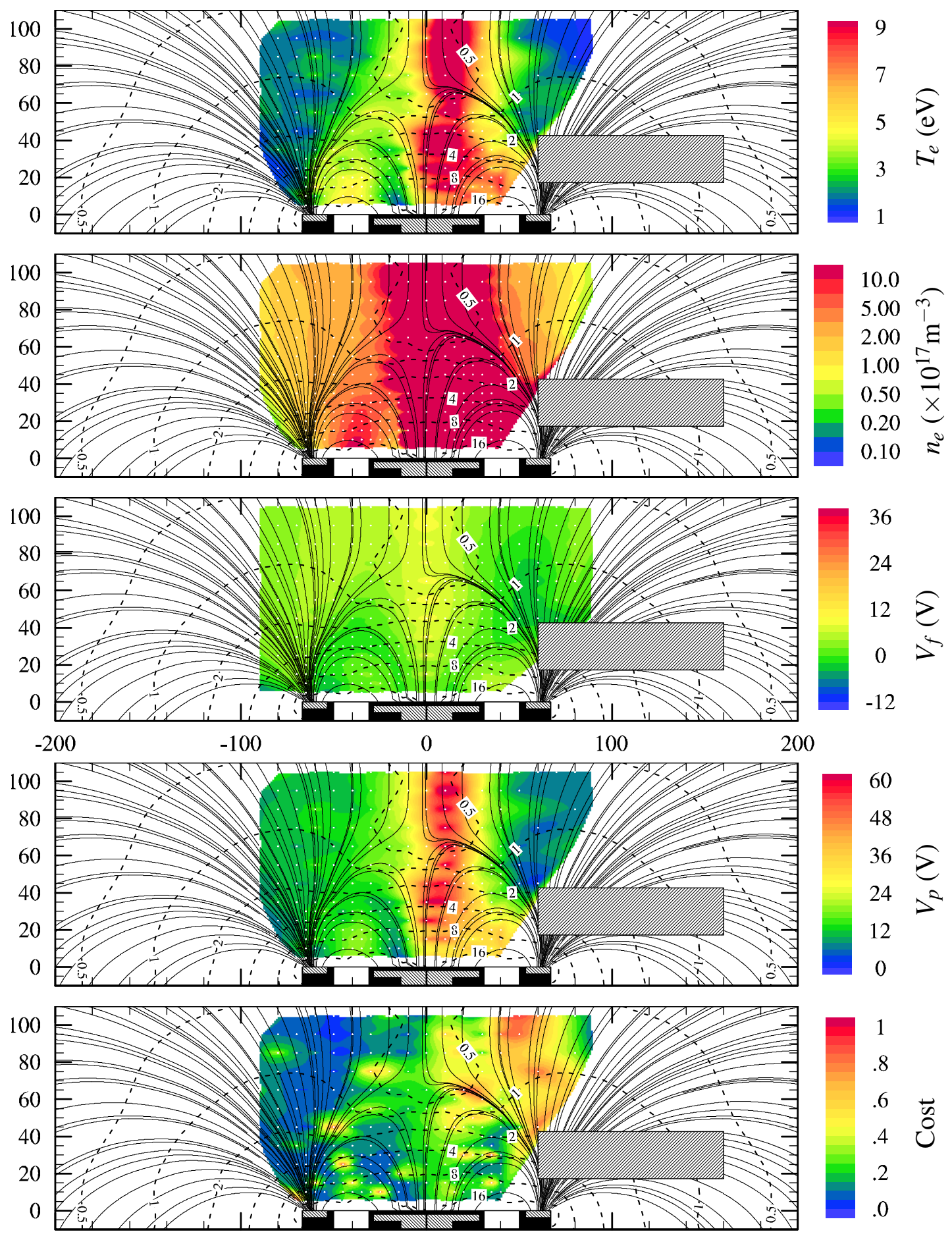

Figure C.37: Plasma properties for Xe with $\dot{m}_{c}=5 \mathrm{SCCM}, r=60 \mathrm{~mm}$ 

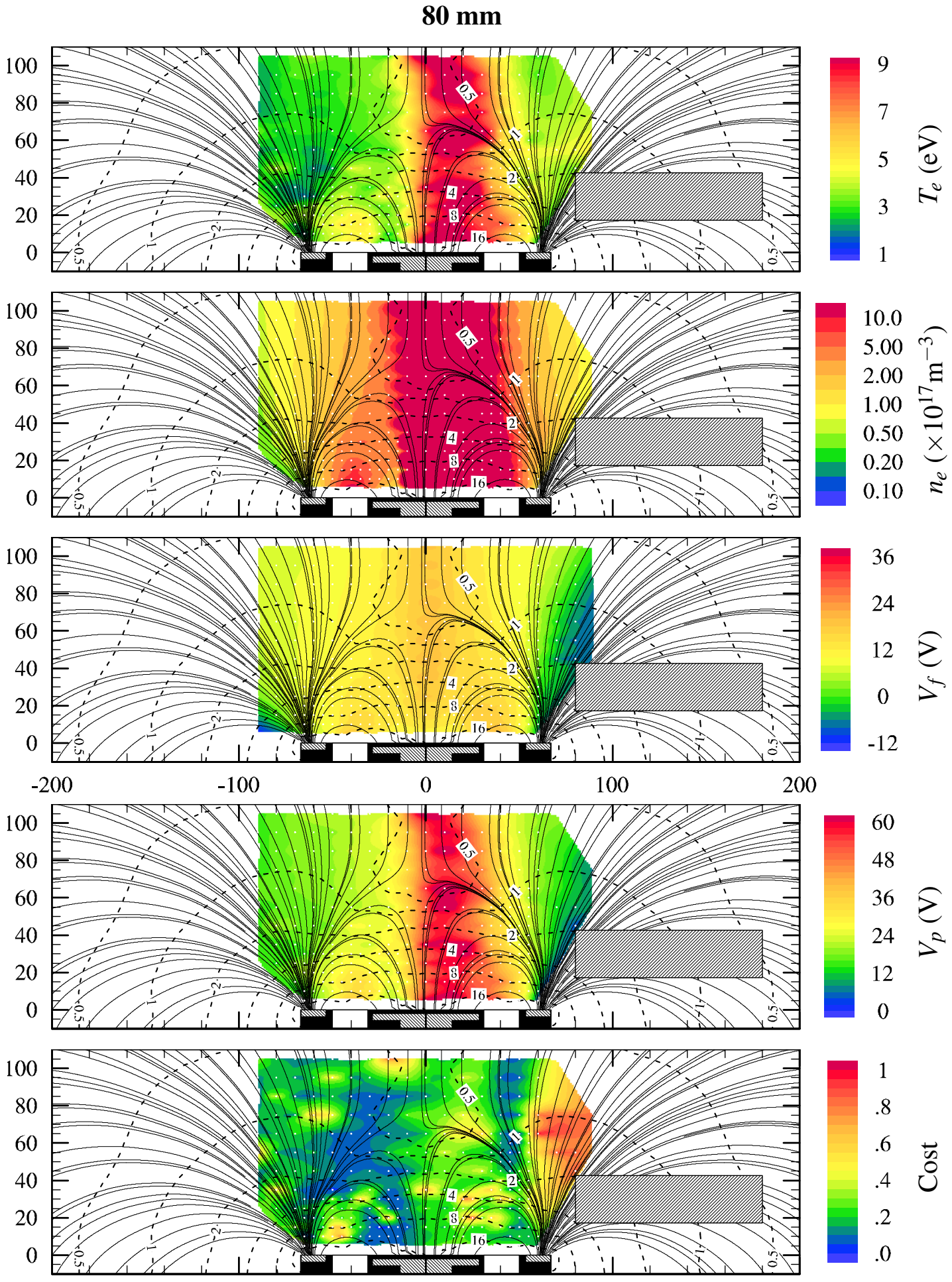

Figure C.38: Plasma properties for Xe with $\dot{m}_{c}=5 \mathrm{SCCM}, r=80 \mathrm{~mm}$ 


\section{$120 \mathrm{~mm}$}
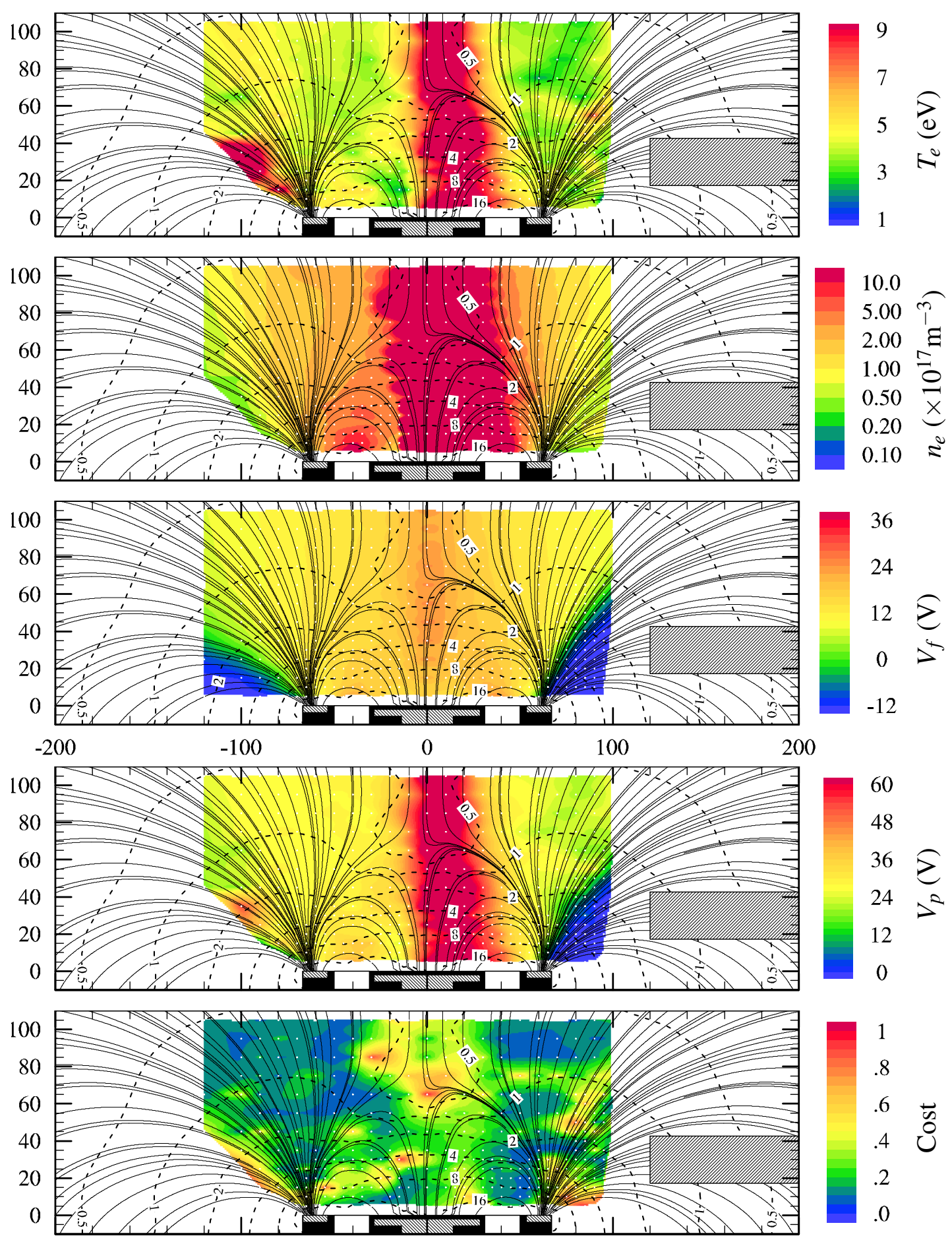

Figure C.39: Plasma properties for Xe with $\dot{m}_{c}=5$ SCCM, $r=120 \mathrm{~mm}$ 

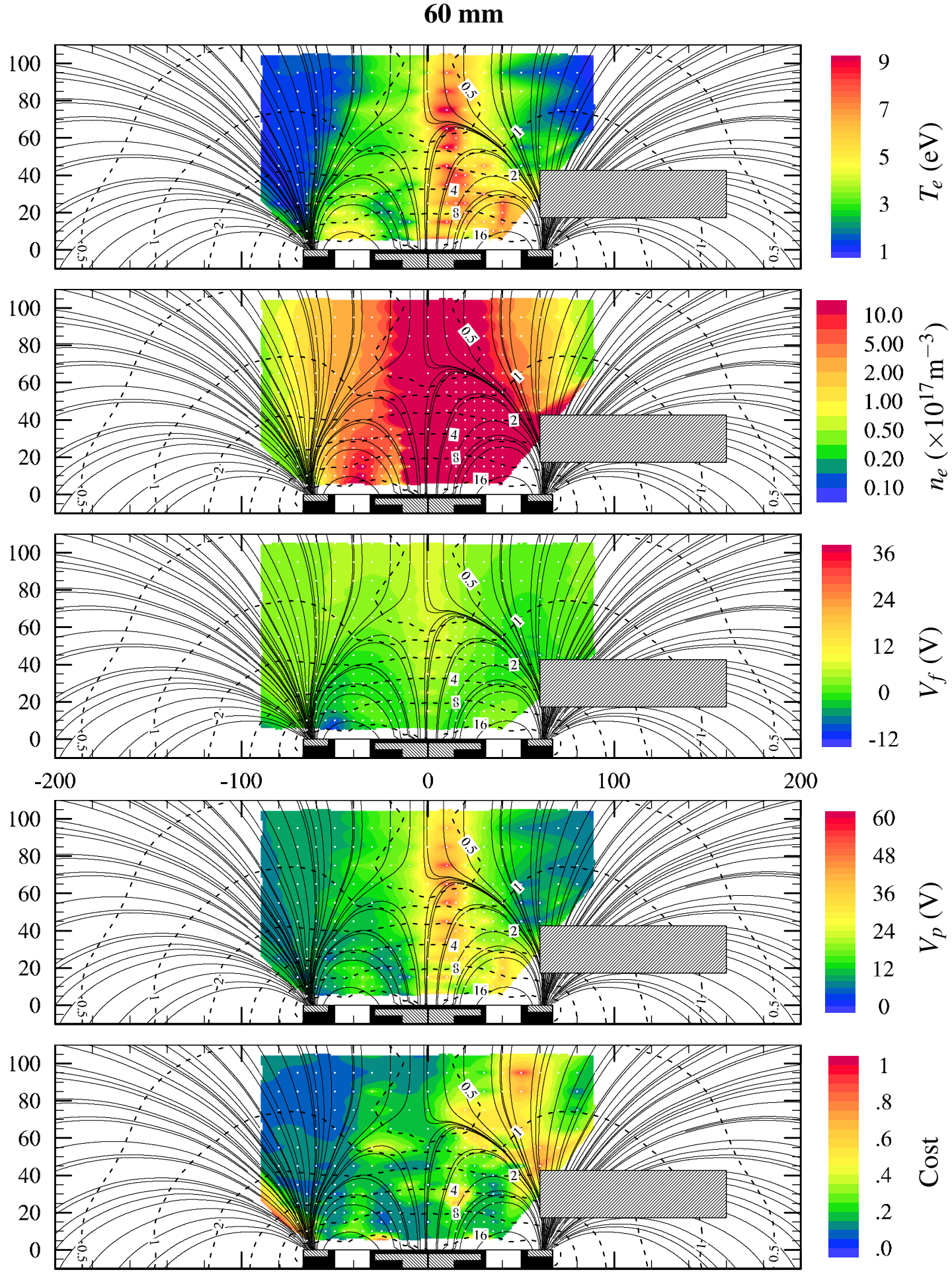

Figure C.40: Plasma properties for Xe with $\dot{m}_{c}=10 \mathrm{SCCM}, r=60 \mathrm{~mm}$ 
$80 \mathrm{~mm}$
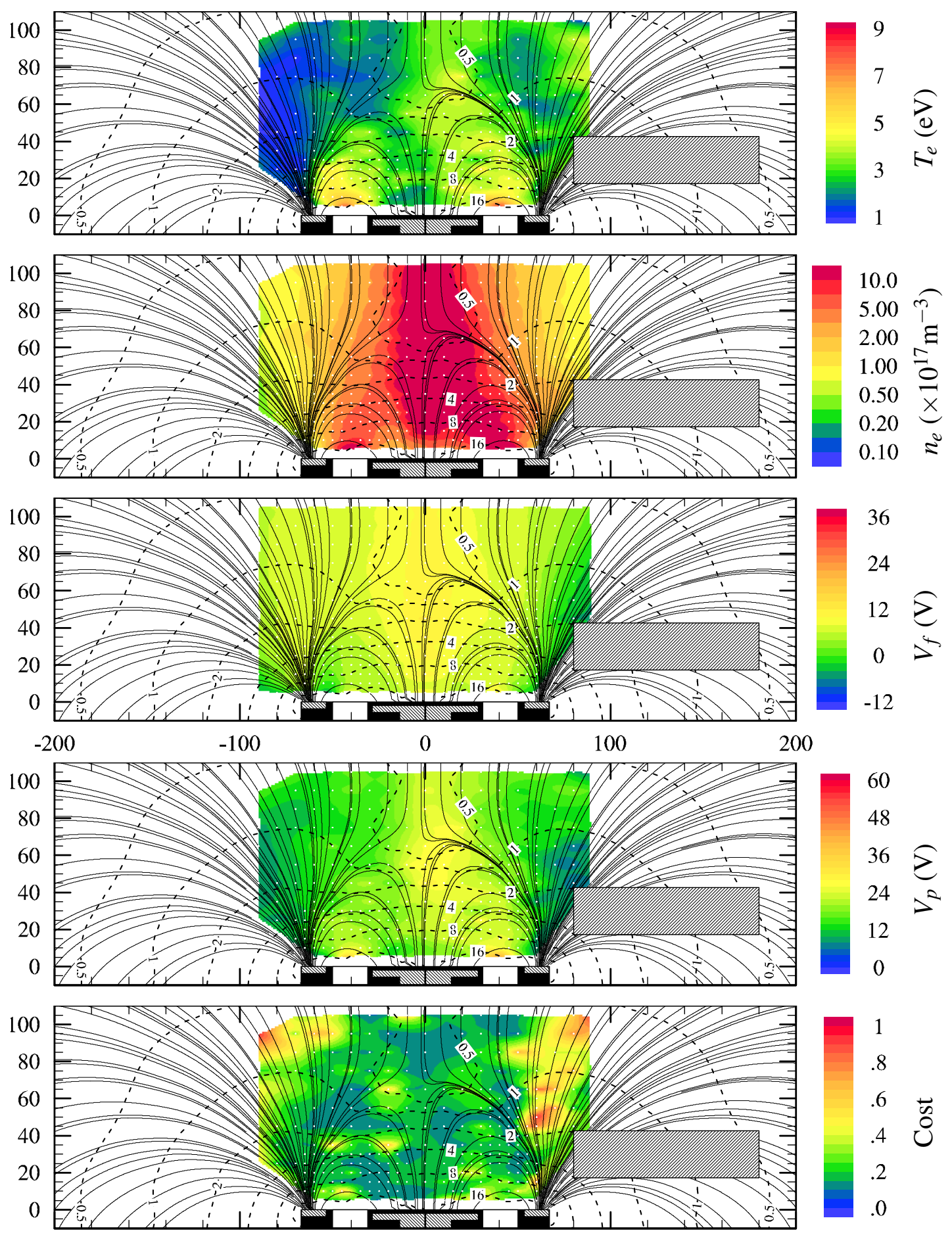

Figure C.41: Plasma properties for Xe with $\dot{m}_{c}=10 \mathrm{SCCM}, r=80 \mathrm{~mm}$ 
$120 \mathrm{~mm}$
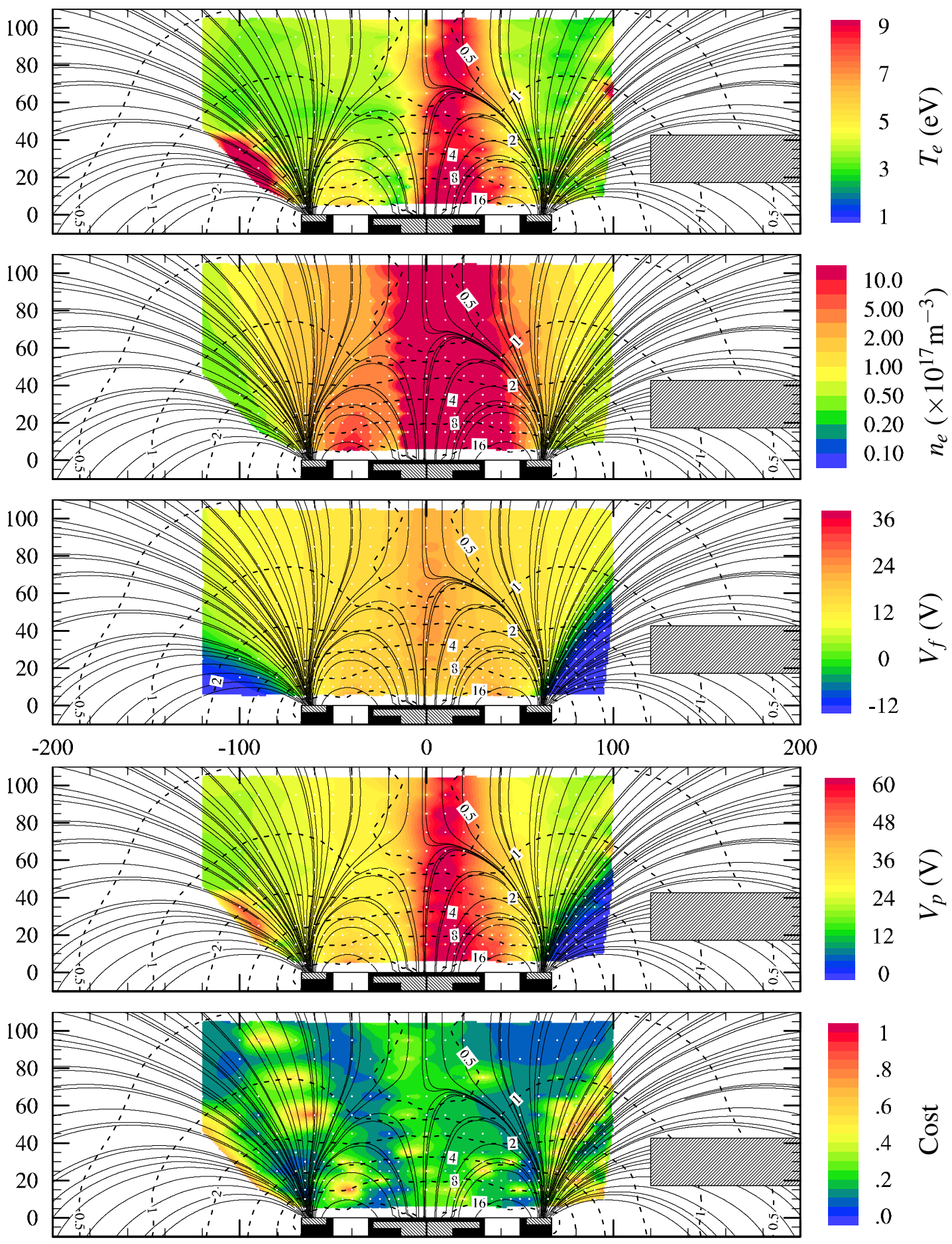

Figure C.42: Plasma properties for Xe with $\dot{m}_{c}=10$ SCCM, $r=120 \mathrm{~mm}$ 


\section{Appendix D}

\section{Xenon Results for the Separatrix Crossing Experiment}

\section{D.1 Performance}

The thruster performance data as a function of cathode position are plotted for the three cathode mass flow rates in Figure D.1. The data show a consistent trend with thrust, cathode coupling voltage, and efficiency highest near thrust-centerline, and falling off to $\sim 120 \mathrm{~mm}$. Beyond this point, these parameters rise slightly. The current, meanwhile, decays rapidly from $40-80 \mathrm{~mm}$, after which it is constant. The decay is more rapid as cathode mass flow decreases.

\section{D.2 Faraday Probe Data}

The current density as a function of angle is plotted in Figure D.2. The data are plotted on a polar graph. However, note there is a change of units at $r=250 \mathrm{~mm}$. Inside this region, a representation of the thruster and cathode are plotted for reference. Outside, the radial dimension corresponds to current density. Each subfigure groups together the data taken at the same radial position but different cathode mass flow rates.

One notes an increased broadening and decreased center-spike magnitude as the cathode is moved away from the thrust axis. There is also a decrease in the overall magnitude of the current densities with decreasing cathode mass flow, though this is less pronounced when the cathode is further from the thrust axis. 


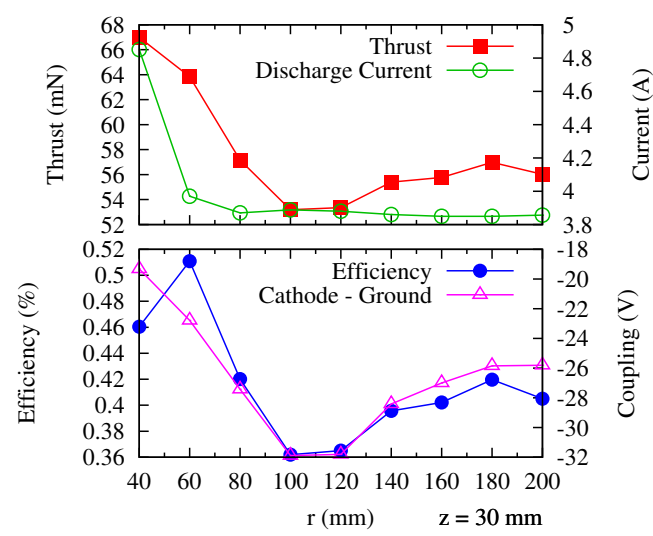

(a) $\dot{m}_{c}=2 \mathrm{SCCM}$

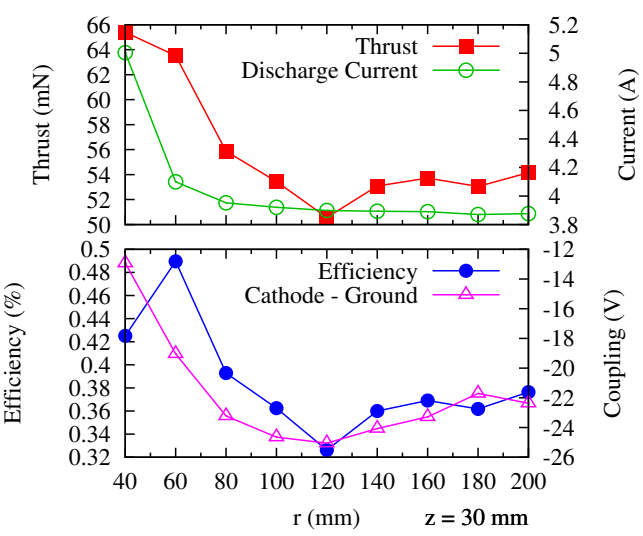

(b) $\dot{m}_{c}=5 \mathrm{SCCM}$

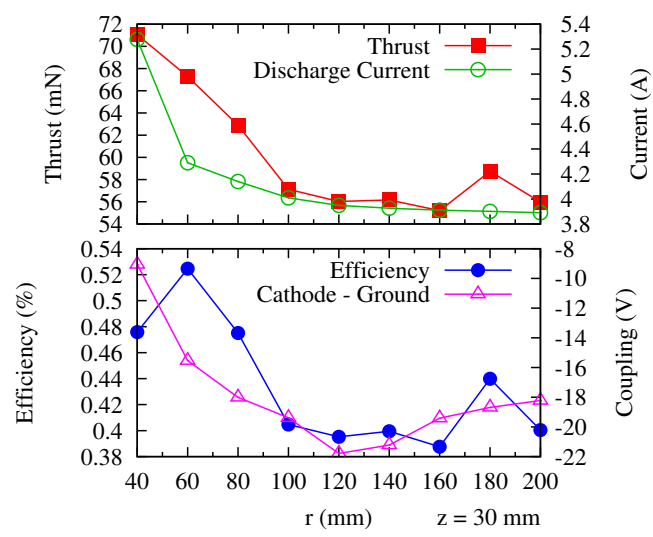

(c) $\dot{m}_{c}=10 \mathrm{SCCM}$

Figure D.1: Thruster performance as a function of cathode position. 


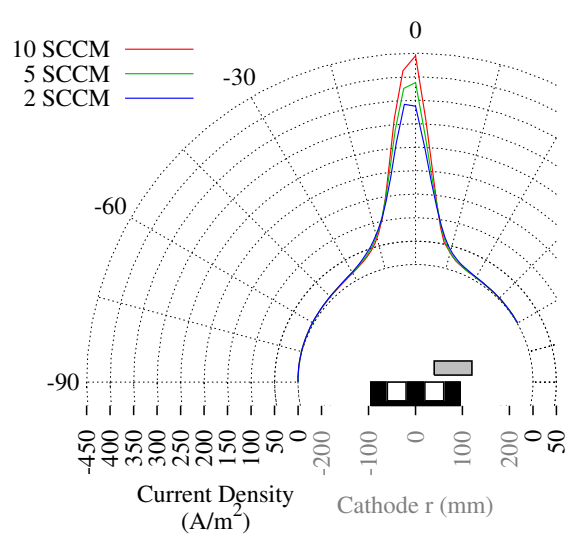

(a) Cathode $\mathrm{r}=40 \mathrm{~mm}$

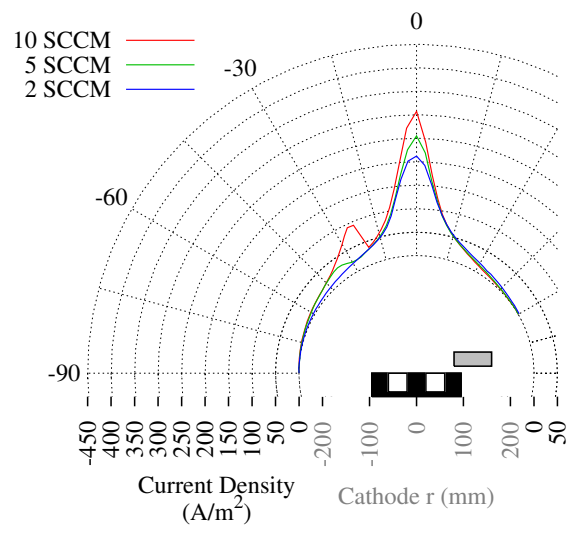

(c) Cathode $\mathrm{r}=80 \mathrm{~mm}$

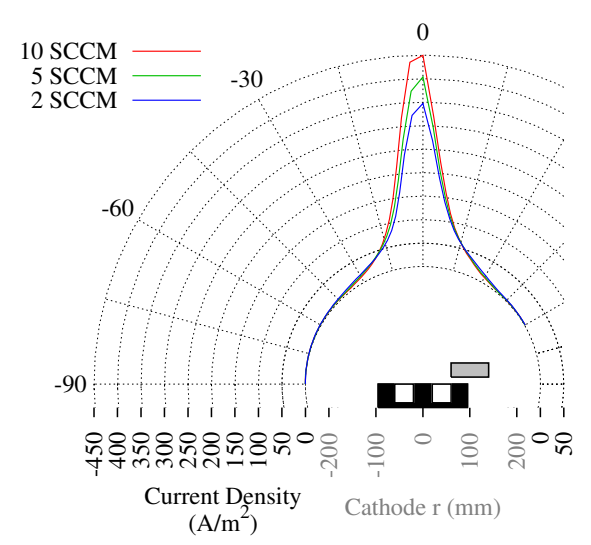

(b) Cathode $\mathrm{r}=60 \mathrm{~mm}$

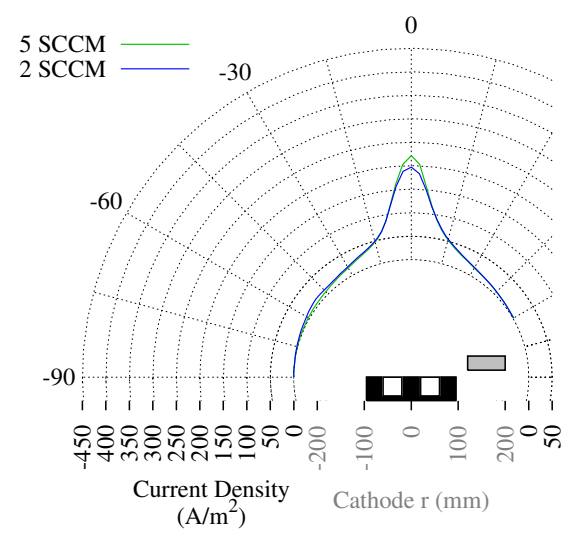

(d) Cathode $\mathrm{r}=120 \mathrm{~mm}$

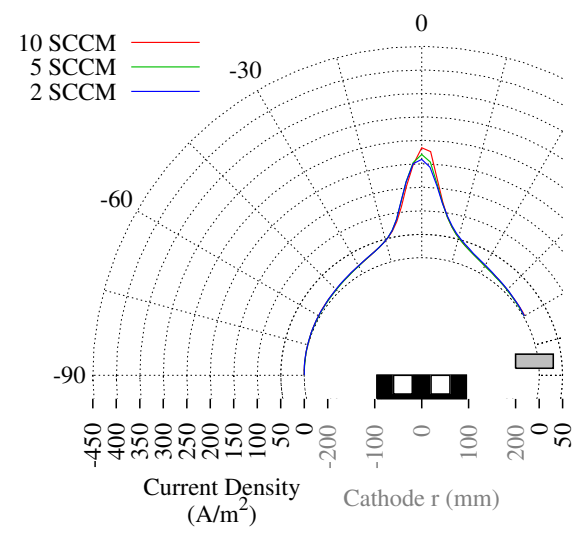

(e) Cathode $\mathrm{r}=200 \mathrm{~mm}$

Figure D.2: Current densities for select cathode positions 


\section{D.3 RPA Data}

Figures D.3 through D.5 show the IEDFs as a function of angle for each cathode position and mass flow operating point measured. Roughly speaking, it shows the probability of an ion at a given angle having an energy that corresponds to the radial dimension. To calculate the total probability, one would need to multiply the probability on the IEDF by the normalized current density at that angle.

The data were taken at 10-degree intervals from -90 degrees to 30 degrees. The remaining data have been interpolated in the figures. The corresponding current density as measured by the Faraday probe is overlaid. Note that the arrangement of the radial axis is identical to that of Figure D.2.

Several of the plots show broad energy distributions at zero degrees. It is possible that these are due to pressure effects inside the RPA and may not, therefore, represent valid data. This will be further addressed in the discussion.

The most striking feature of these graphs is that as the cathode is moved away from the thruster, the distributions become narrower in energy space. Furthermore, the broad energy distribution at 0 degrees seems to go away as the cathode is moved away from the thrust axis and as cathode mass flow is decreased. When present, the narrow spike in probability density occurs at approximately $200 \mathrm{~V}$, regardless of cathode position, and extends out to about -60 degrees. It appears to vary slightly with cathode mass flow, moving from $\sim 200 \mathrm{~V}$ to $\sim 210 \mathrm{~V}$ as the mass flow is varied from 2 SCCM to 10 SCCM.

Finally, one notes a low energy spike near -90 degrees. However, signal levels here are quite small, and we have low confidence in the data taken between -80 and -90 degrees.

\section{D.4 Efficiency Analysis}

The calculated efficiencies show good agreement with the measured efficiencies, except when $\dot{m}_{c}=10 \mathrm{SCCM}$ and at all cathode mass flow rates when $r=60 \mathrm{~mm}$. The reasons for these discrepancies are unclear. The voltage utilization efficiency is the dominant loss mechanism, with beam divergence being a close second. The remaining efficiency components are all $\sim 85 \%$ or better. All efficiency components show similar trends as cathode position is varied, regardless of cathode mass flow. The consistency of the trends suggests that there is no fundamental difference in thruster operation as a function of cathode mass flow rate.

The voltage utilization efficiency increases as the cathode is moved away from the thruster. This is likely explained by the decrease in cathode propellent ingestion as $r$ increases. Ingested cathode propellent is more likely to be ionized further down the potential hill and across a greater range of potentials, as it comes from outside the 


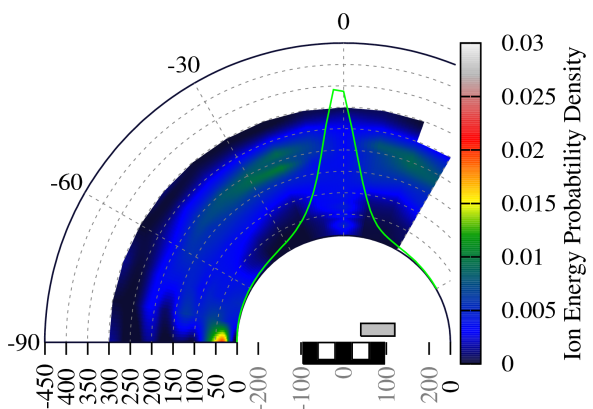

Current Density $\left(\mathrm{A} / \mathrm{m}^{2}\right)$ Cathode $\mathrm{r}(\mathrm{mm})$
Ion E/q (V)

(a) $\dot{m}_{c}=2 \mathrm{SCCM}$, Cathode $\mathrm{r}=40 \mathrm{~mm}$

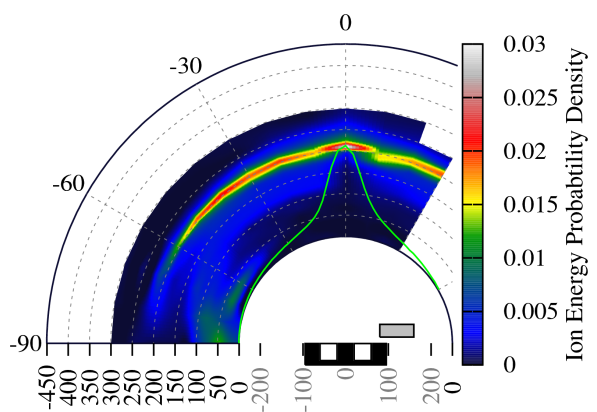

Current Density $\left(\mathrm{A} / \mathrm{m}^{2}\right)$ Cathode r $(\mathrm{mm})$

(c) $\dot{m}_{c}=2 \mathrm{SCCM}$, Cathode $\mathrm{r}=80 \mathrm{~mm}$

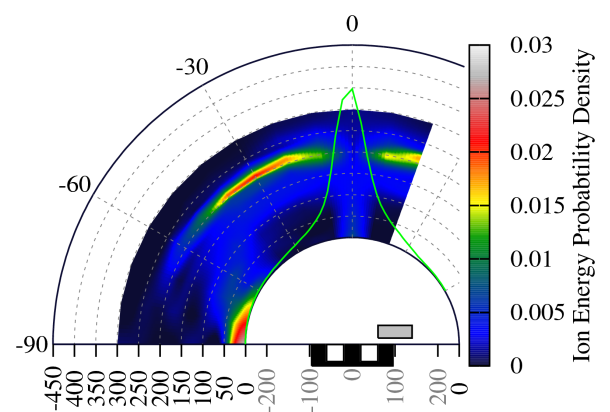

Current Density $\left(\mathrm{A} / \mathrm{m}^{2}\right)$ Cathode $\mathrm{r}(\mathrm{mm})$
Ion E/q $(\mathrm{V})$

(b) $\dot{m}_{c}=2$ SCCM, Cathode $\mathrm{r}=60 \mathrm{~mm}$

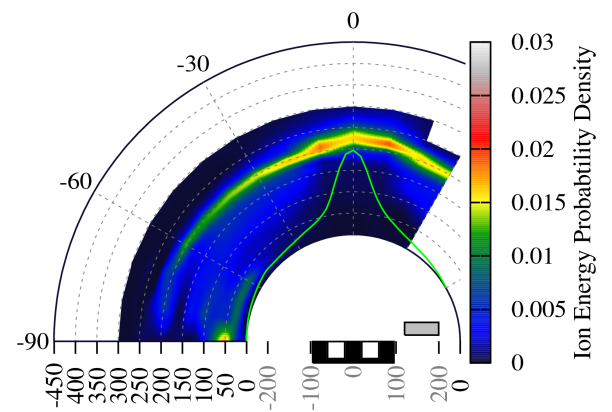

Current Density $\left(\mathrm{A} / \mathrm{m}^{2}\right)$ Cathode $\mathrm{r}(\mathrm{mm})$

(d) $\dot{m}_{c}=2$ SCCM, Cathode $\mathrm{r}=120 \mathrm{~mm}$

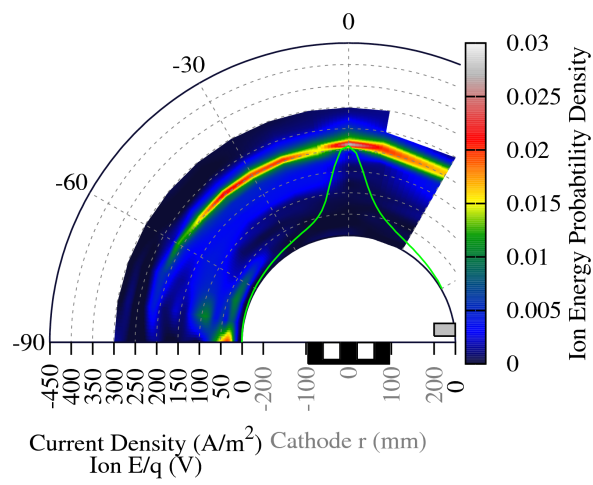

(e) $\dot{m}_{c}=2 \mathrm{SCCM}$, Cathode $\mathrm{r}=200 \mathrm{~mm}$

Figure D.3: Ion energy distributions and ion current density as a function of angle for cathode mass flow rates of $2 \mathrm{SCCM}$ 


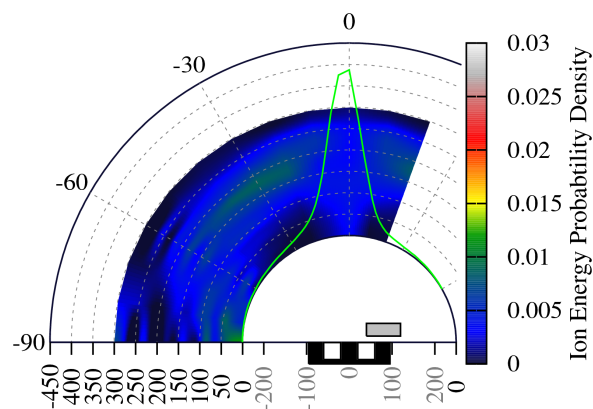

Current Density $\left(\mathrm{A} / \mathrm{m}^{2}\right)$ Cathode $\mathrm{r}(\mathrm{mm})$
Ion E/q $(\mathrm{V})$

(a) $\dot{m}_{c}=5 \mathrm{SCCM}$, Cathode $\mathrm{r}=40 \mathrm{~mm}$

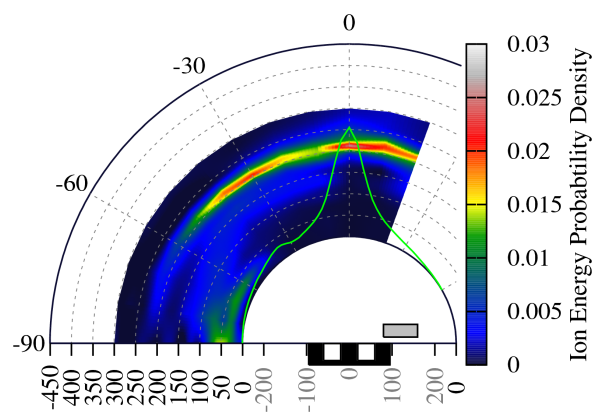

Current Density $\left(\mathrm{A} / \mathrm{m}^{2}\right)$ Cathode $\mathrm{r}(\mathrm{mm})$

(c) $\dot{m}_{c}=5$ SCCM, Cathode $\mathrm{r}=80 \mathrm{~mm}$

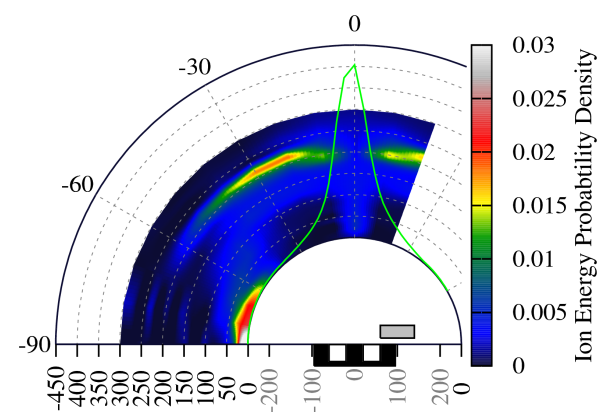

Current Density $\left(\mathrm{A} / \mathrm{m}^{2}\right)$ Cathode $\mathrm{r}(\mathrm{mm})$
Ion $\mathrm{E} / \mathrm{q}(\mathrm{V})$

(b) $\dot{m}_{c}=5$ SCCM, Cathode $\mathrm{r}=60 \mathrm{~mm}$

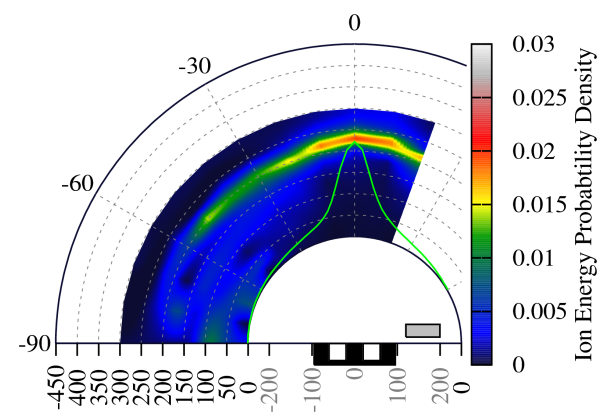

Current Density (A/m²) Cathode r (mm)

(d) $\dot{m}_{c}=5$ SCCM, Cathode $\mathrm{r}=120 \mathrm{~mm}$

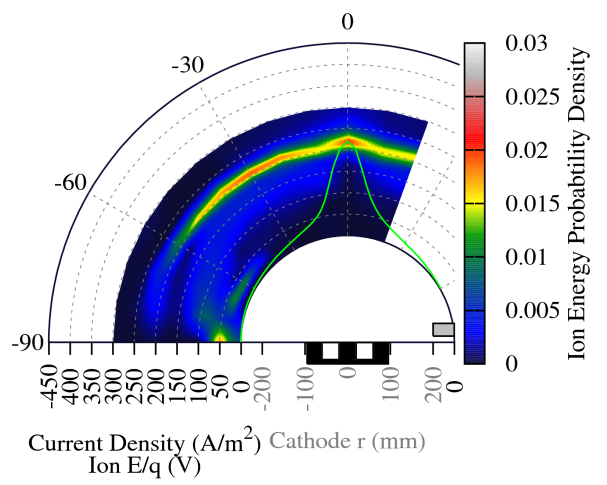

(e) $\dot{m}_{c}=5$ SCCM, Cathode $\mathrm{r}=200 \mathrm{~mm}$

Figure D.4: Ion energy distributions and ion current density as a function of angle for cathode mass flow rates of $5 \mathrm{SCCM}$ 


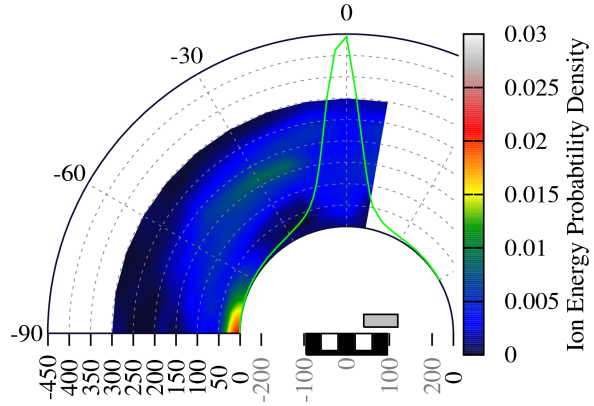

Current Density $\left(\mathrm{A} / \mathrm{m}^{2}\right)$ Cathode $\mathrm{r}(\mathrm{mm})$
Ion E/q $(\mathrm{V})$

(a) $\dot{m}_{c}=10 \mathrm{SCCM}$, Cathode $\mathrm{r}=40 \mathrm{~mm}$

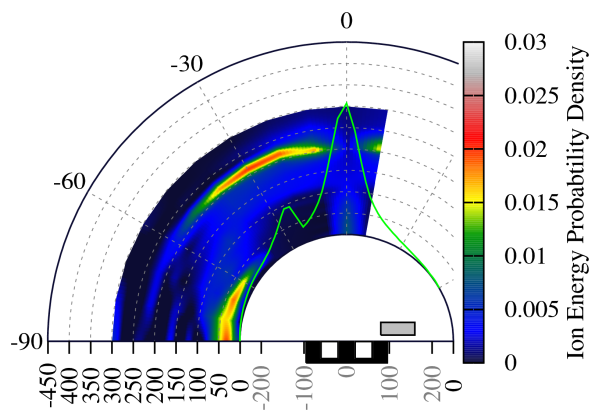

Current Density $\left(\mathrm{A} / \mathrm{m}^{2}\right)$ Cathode r $(\mathrm{mm})$
Ion E/q $(\mathrm{V})$

(c) $\dot{m}_{c}=10$ SCCM, Cathode $\mathrm{r}=80 \mathrm{~mm}$

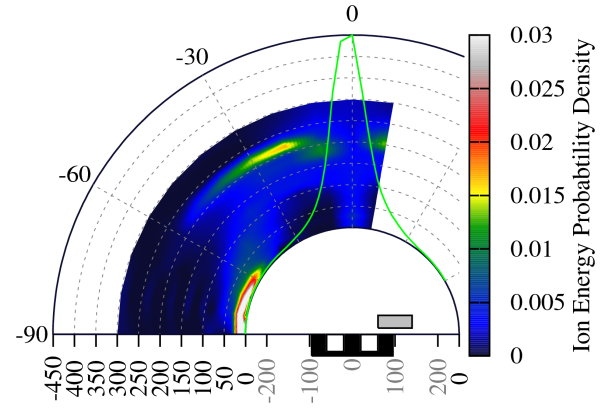

Current Density $\left(\mathrm{A} / \mathrm{m}^{2}\right)$ Cathode $\mathrm{r}(\mathrm{mm})$
Ion E/q $(\mathrm{V})$

(b) $\dot{m}_{c}=10$ SCCM, Cathode r $=60 \mathrm{~mm}$

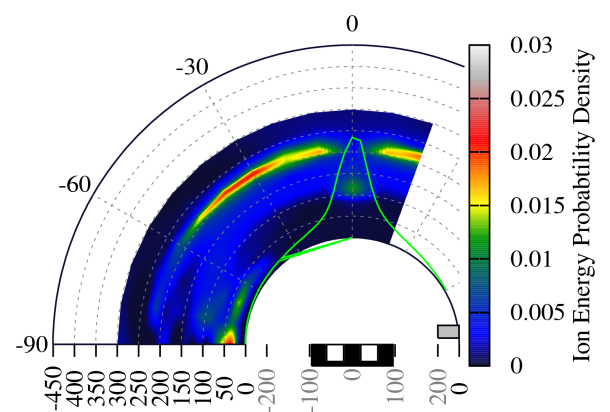

Current Density $\left(\mathrm{A} / \mathrm{m}^{2}\right)$
Ion E/q (V) Cathode r ( $\mathrm{mm})$

(d) $\dot{m}_{c}=10$ SCCM, Cathode $\mathrm{r}=200 \mathrm{~mm}$

Figure D.5: Ion energy distributions and ion current density as a function of angle for cathode mass flow rates of $10 \mathrm{SCCM}$ 


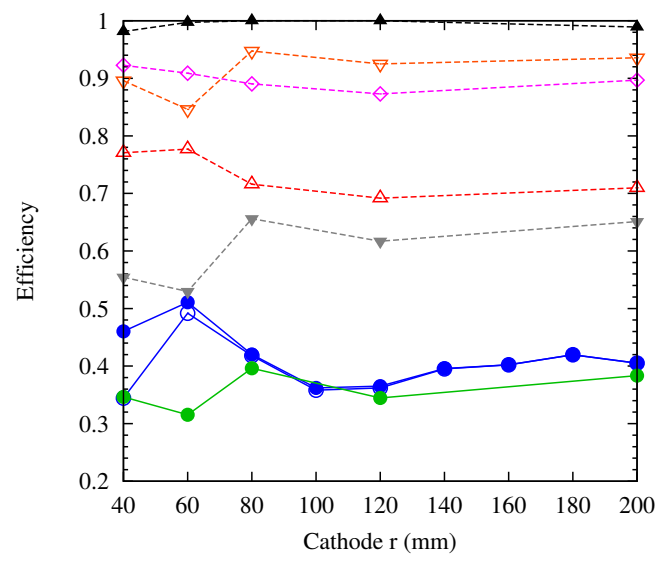

(a) $\dot{m}_{c}=2 \mathrm{SCCM}$

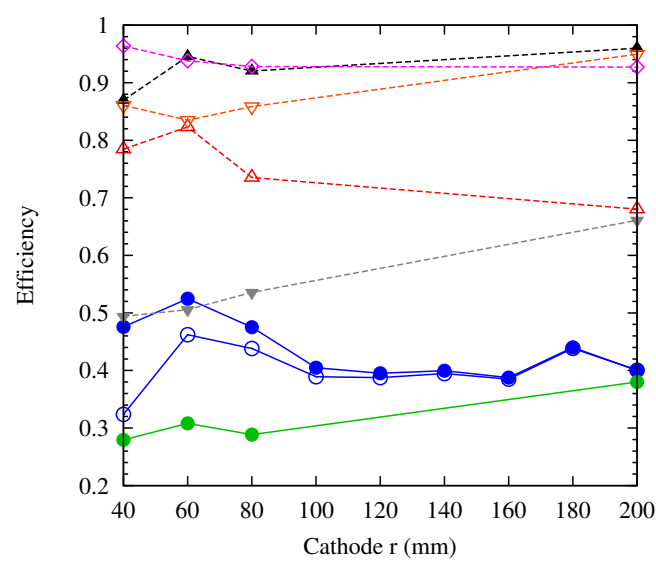

(c) $\dot{m}_{c}=10 \mathrm{SCCM}$

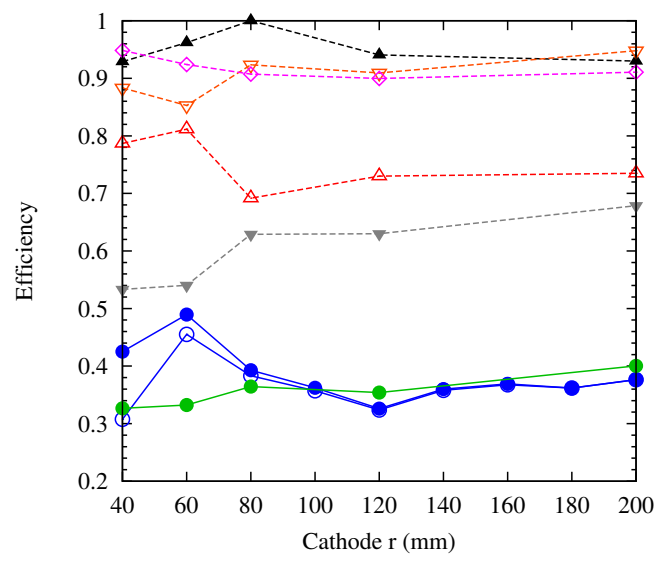

(b) $\dot{m}_{c}=5 \mathrm{SCCM}$

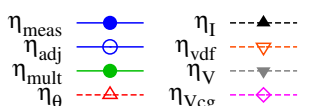

Figure D.6: Efficiency decomposition versus cathode radial location 
thruster, rather than the back of the anode. An increase in cathode propellent ingestion would, therefore, result in a decrease in voltage utilization, as the velocity distribution was spread to lower values. The fact that the velocity distribution efficiency trends in the same fashion as the voltage utilization also supports this theory, as it measures the spread in velocity space of the beam ions.

The current utilization efficiency is quite high, and relatively flat. It does drop significantly at $40 \mathrm{~mm}$, which in part explains the consistently poor performance at this point. Given the high, periodic oscillations in cathode coupling voltage observed, it is possible that there was increased electron mobility in the HET channel, which would exhibit itself as a decrease in current utilization efficiency. A more interesting trend is seen when comparing the overall current utilization from each of the cathode mass flow rates. As cathode mass flow rate increases, current utilization decreases. One possible explanation is that the increased cathode flow results in increased charge-exchange collisions. The fast neutrals, which must be captured for a completely accurate picture of current utilization, are not detected by the Faraday probe. On the other hand, if this were the only effect, one would expect the current utilization to be much worse with the cathode close to the thruster, than with it at $r=200 \mathrm{~mm}$. This is not the case. But, increased ionization of the cathode propellent may offset this, making the two effects impossible to disentangle with the current data.

The cathode coupling efficiency trends nicely match the trends in efficiency. This can be seen in Figure D.1, where $V_{\mathrm{cg}}\left(\propto \eta_{\mathrm{Vcg}}\right)$ is seen to vary nearly in step with performance. However, cathode coupling efficiencies only vary by roughly $5 \%$ as the cathode position is changed, while total efficiencies vary by up to $20 \%$. We see that the beam divergence efficiency shows roughly the same trend as the overall efficiency, and also approximately matches the coupling voltage efficiency trends. The divergence efficiency exhibits a maximum change of roughly $10 \%$ as cathode position changes. It is surprising that even at $40 \mathrm{~mm}$, where the cathode partially blocks the thruster channel, beam divergence remains good. As previously mentioned, the trend seen here is further supported by the conclusions of Hofer et al. ${ }^{1}$ Clearly beam divergence is an important factor in explaining the trend in total efficiency.

\section{References for Appendix D}

[1] Hofer, R. R., Johnson, L. K., Goebel, D. M., and Fitzgerald, D. J., "Effects of an Internally-Mounted Cathode on Hall Thruster Plume Properties," 42nd AIAA/ASME/SAE/ASEE Joint Propulsion Conference, July 9-12, 2006, Paper No. AIAA2006-4482 


\section{Appendix E}

\section{Single-Point Data}

This appendix presents a table of all of the scalar quanties measured at each of the combinations of pole, cathode mass flow rate, and cathode position measured in the separatrix crossing experiment of Chapter 8. All of the parameters are explained and ncertainty estimates for most of the parameters are given in that chapter. See Table 8.2 for further explanations of Order and the scalar plume parameters. 
Table E.1: Scalar data from separatrix crossing experiment

\begin{tabular}{|c|c|c|c|c|c|c|c|c|c|c|c|c|c|c|c|c|c|c|c|}
\hline \multicolumn{4}{|c|}{ Independent Variables } & \multicolumn{3}{|c|}{ Performance } & \multicolumn{2}{|c|}{ Beam } & \multicolumn{4}{|c|}{ Plume } & \multicolumn{5}{|c|}{ Efficiency Components } & \multicolumn{2}{|c|}{ Efficiency } \\
\hline Pole & $\begin{array}{c}\dot{m}_{c} \\
(\mathrm{SCCM})\end{array}$ & $\begin{array}{c}\mathrm{r} \\
(\mathrm{mm})\end{array}$ & Order & $\begin{array}{l}\text { Thrust } \\
(\mathrm{mN})\end{array}$ & $\begin{array}{l}I_{d} \\
\text { (A) }\end{array}$ & $\begin{array}{l}V_{\mathrm{cg}} \\
(\mathrm{V})\end{array}$ & $\begin{array}{l}I_{b} \\
\text { (A) }\end{array}$ & $\begin{array}{l}\langle|\theta|\rangle \\
\left(^{\circ}\right)\end{array}$ & $\begin{array}{l}\overline{\nabla_{p}} \\
(\mathrm{~V})\end{array}$ & $\begin{array}{l}V_{f} \\
(\mathrm{~V})\end{array}$ & $\begin{array}{c}\overline{T_{e}} \\
(\mathrm{eV})\end{array}$ & $\begin{array}{c}\bar{n}_{e} \\
\left(\times 10^{17} \mathrm{~m}^{-3}\right)\end{array}$ & $\eta_{\theta}$ & $\eta_{I}$ & $\eta_{\mathrm{Vcg}}$ & $\eta_{\mathrm{vdf}}$ & $\eta_{V}$ & $\eta_{a}$ & $\eta_{t}$ \\
\hline $\mathrm{OOP}$ & 2 & 40 & 2 & 26.5 & 3.95 & 32.7 & 2.81 & 31.9 & & & & & 0.635 & 0.712 & 0.868 & 0.935 & 0.584 & 0.139 & 0.132 \\
\hline OOP & 2 & 50 & 8 & 25.3 & 3.36 & 26.4 & 2.61 & 28.4 & 2.72 & 28.5 & 5.86 & 3.62 & 0.689 & 0.775 & 0.894 & 0.879 & 0.487 & 0.148 & 0.141 \\
\hline OOP & 2 & 60 & 5 & 25.1 & 3.15 & 29.8 & & & 0.438 & 32.7 & 6.08 & 3.16 & & & & & & & \\
\hline $\mathrm{OOP}$ & 2 & 70 & 10 & 22.4 & 2.93 & 41.0 & 2.56 & 34.5 & & & & & 0.598 & 0.876 & 0.835 & 0.947 & 0.559 & 0.134 & 0.127 \\
\hline $\mathrm{OOP}$ & 2 & 80 & 6 & 20.4 & 2.84 & 48.6 & 2.56 & 34.9 & 11.8 & 42.8 & 5.79 & 3.58 & 0.597 & 0.902 & 0.805 & 0.915 & 0.558 & 0.114 & 0.109 \\
\hline $\mathrm{OOP}$ & 2 & 90 & 9 & 21.9 & 2.91 & 46.1 & 2.41 & 38.7 & & & & & 0.540 & 0.828 & 0.815 & 0.953 & 0.598 & 0.128 & 0.122 \\
\hline $\mathrm{OOP}$ & 2 & 100 & 1 & 20.0 & 3.03 & 46.2 & 2.08 & 36.8 & 23.0 & 63.0 & 7.56 & 3.01 & 0.568 & 0.687 & 0.815 & 0.965 & 0.610 & 0.103 & 0.0982 \\
\hline $\mathrm{OOP}$ & 2 & 120 & 7 & 19.9 & 3.00 & 48.7 & 2.02 & 37.1 & & & & & 0.566 & 0.675 & 0.805 & 0.983 & 0.613 & 0.103 & 0.0982 \\
\hline OOP & 2 & 180 & 4 & 20.7 & 2.96 & 47.9 & & & & & & & & & & & & & \\
\hline $\mathrm{OOP}$ & 5 & 40 & 3 & 28.2 & 4.22 & 24.0 & 2.64 & 29.5 & & & & & 0.674 & 0.626 & 0.903 & 0.942 & 0.606 & 0.147 & 0.131 \\
\hline $\mathrm{OOP}$ & 5 & 50 & 11 & 25.2 & 3.66 & 19.8 & 2.56 & 26.6 & 0.503 & 26.7 & 5.08 & 3.71 & 0.717 & 0.699 & 0.920 & 0.909 & 0.522 & 0.135 & 0.120 \\
\hline OOP & 5 & 60 & 6 & 25.1 & 3.53 & 19.9 & 2.71 & 27.7 & 1.15 & 28.3 & 5.10 & 3.23 & 0.702 & 0.769 & 0.920 & 0.888 & 0.492 & 0.139 & 0.124 \\
\hline OOP & 5 & 70 & 14 & 21.6 & 3.18 & 25.3 & 2.80 & 32.1 & & & & & 0.634 & 0.881 & 0.898 & 0.918 & 0.530 & 0.114 & 0.102 \\
\hline OOP & 5 & 80 & 8 & 20.9 & 3.04 & 33.3 & 2.78 & 35.7 & 7.51 & 34.9 & 5.12 & 3.62 & 0.580 & 0.917 & 0.866 & 0.894 & 0.496 & 0.112 & 0.100 \\
\hline ООР & 5 & 90 & 13 & 18.8 & 2.96 & 38.2 & 2.57 & 33.7 & & & & & 0.616 & 0.865 & 0.847 & 0.955 & 0.599 & 0.0932 & 0.0831 \\
\hline $\mathrm{OOP}$ & 5 & 100 & 2 & 21.6 & 2.93 & 41.2 & 2.40 & 33.7 & 14.6 & 49.6 & 6.56 & 3.65 & 0.619 & 0.817 & 0.835 & 0.963 & 0.619 & 0.124 & 0.111 \\
\hline $\mathrm{OOP}$ & 5 & 120 & 5 & 19.4 & 2.99 & 39.0 & 2.15 & 33.1 & 18.4 & 53.6 & 6.67 & 2.69 & 0.630 & 0.719 & 0.843 & 0.977 & 0.630 & 0.0982 & 0.0876 \\
\hline OOP & 5 & 140 & 9 & 18.9 & 3.03 & 39.4 & & & & & & & & & & & & & \\
\hline $\mathrm{OOP}$ & 5 & 160 & 7 & 20.1 & 3.09 & 38.2 & & & & & & & & & & & & & \\
\hline OOP & 5 & 180 & 10 & 19.9 & 3.10 & 38.9 & & & & & & & & & & & & & \\
\hline OOP & 5 & 200 & 4 & 22.4 & 3.06 & 37.7 & 2.29 & 39.1 & 15.5 & 52.5 & 6.97 & 3.36 & 0.536 & 0.747 & 0.849 & 0.963 & 0.602 & 0.128 & 0.114 \\
\hline $\mathrm{OOP}$ & 5 & 250 & 12 & 21.2 & 3.01 & 38.1 & & & & & & & & & & & & & \\
\hline $\mathrm{OOP}$ & 10 & 40 & 2 & 32.4 & 4.38 & 25.6 & 2.85 & 29.5 & & & & & 0.675 & 0.651 & 0.897 & 0.889 & 0.545 & 0.187 & 0.150 \\
\hline OOP & 10 & 50 & 10 & 27.5 & 4.03 & 17.1 & 2.78 & 26.6 & 0.244 & 21.4 & 4.03 & 3.67 & 0.717 & 0.689 & 0.931 & 0.865 & 0.477 & 0.146 & 0.118 \\
\hline $\mathrm{OOP}$ & 10 & 60 & 5 & 28.2 & 3.98 & 15.9 & 3.00 & 28.5 & 0.520 & 21.1 & 3.86 & 2.95 & 0.688 & 0.754 & 0.936 & 0.863 & 0.462 & 0.155 & 0.125 \\
\hline $\mathrm{OOP}$ & 10 & 70 & 13 & 26.0 & 3.57 & 19.9 & 2.93 & 30.3 & & & & & 0.662 & 0.821 & 0.920 & 0.841 & 0.442 & 0.147 & 0.118 \\
\hline OOP & 10 & 80 & 7 & 25.1 & 3.37 & 23.1 & 2.95 & 33.1 & 4.61 & 24.9 & 3.76 & 3.53 & 0.620 & 0.875 & 0.907 & 0.864 & 0.471 & 0.145 & 0.117 \\
\hline $\mathrm{OOP}$ & 10 & 90 & 12 & 22.7 & 3.20 & 26.4 & 2.88 & 34.5 & & & & & 0.599 & 0.900 & 0.894 & 0.881 & 0.490 & 0.125 & 0.101 \\
\hline $\mathrm{OOP}$ & 10 & 100 & 4 & 23.8 & 3.16 & 29.2 & 2.91 & 36.0 & 9.13 & 34.4 & 4.72 & 3.40 & 0.574 & 0.921 & 0.883 & 0.892 & 0.511 & 0.139 & 0.112 \\
\hline OOP & 10 & 120 & 1 & 24.2 & 3.08 & 30.7 & 2.66 & 34.0 & 10.4 & 31.2 & 3.88 & 2.98 & 0.611 & 0.861 & 0.876 & 0.935 & 0.588 & 0.148 & 0.119 \\
\hline OOP & 10 & 140 & 8 & 20.3 & 3.05 & 29.9 & & & & & & & & & & & & & \\
\hline $\mathrm{OOP}$ & 10 & 160 & 6 & 21.3 & 3.03 & 30.5 & & & & & & & & & & & & & \\
\hline OOP & 10 & 180 & 9 & 22.3 & 2.99 & 32.0 & & & & & & & & & & & & & \\
\hline $\mathrm{OOP}$ & 10 & 200 & 3 & 23.3 & 2.99 & 31.6 & 2.66 & 34.2 & 12.2 & 37.1 & 4.64 & 2.98 & 0.607 & 0.888 & 0.873 & 0.967 & 0.624 & 0.141 & 0.113 \\
\hline $\mathrm{OOP}$ & 10 & 250 & 11 & 23.5 & 2.93 & 32.5 & & & & & & & & & & & & & \\
\hline EOP & 2 & 40 & 2 & 30.8 & 4.05 & 30.8 & 2.49 & 28.7 & & & & & 0.694 & 0.616 & 0.876 & 0.948 & 0.653 & 0.183 & 0.174 \\
\hline EOP & 2 & 50 & 4 & 27.3 & 3.38 & 27.8 & 2.32 & 27.7 & 1.22 & 25.5 & 5.01 & 3.43 & 0.705 & 0.687 & 0.888 & 0.864 & 0.503 & 0.172 & 0.164 \\
\hline
\end{tabular}


Table E.1 - continued from previous page

\begin{tabular}{|c|c|c|c|c|c|c|c|c|c|c|c|c|c|c|c|c|c|c|c|}
\hline \multicolumn{4}{|c|}{ Independent Variables } & \multicolumn{3}{|c|}{ Performance } & \multicolumn{2}{|c|}{ Beam } & \multicolumn{4}{|c|}{ Plume } & \multicolumn{5}{|c|}{ Efficiency Components } & \multicolumn{2}{|c|}{ Efficiency } \\
\hline Pole & $\begin{array}{c}\dot{m}_{c} \\
(\mathrm{SCCM})\end{array}$ & $\begin{array}{c}\mathrm{r} \\
(\mathrm{mm})\end{array}$ & Order & $\begin{array}{l}\text { Thrust } \\
(\mathrm{mN})\end{array}$ & $\begin{array}{l}I_{d} \\
(\mathrm{~A})\end{array}$ & $\begin{array}{l}V_{\mathrm{cg}} \\
(\mathrm{V})\end{array}$ & $\begin{array}{l}I_{b} \\
(\mathrm{~A})\end{array}$ & $\begin{array}{l}\langle|\theta|\rangle \\
\left(^{\circ}\right)\end{array}$ & $\begin{array}{l}\overline{V_{p}} \\
(\mathrm{~V})\end{array}$ & $\begin{array}{l}V_{f} \\
(\mathrm{~V})\end{array}$ & $\begin{array}{l}\overline{T_{e}} \\
(\mathrm{eV})\end{array}$ & $\begin{array}{c}\overline{\bar{n}_{e}} \\
\left(\times 10^{17} \mathrm{~m}^{-3}\right)\end{array}$ & $\eta_{\theta}$ & $\eta_{I}$ & $\eta_{\mathrm{Vcg}}$ & $\eta_{\mathrm{vdf}}$ & $\eta_{V}$ & $\eta_{a}$ & $\eta_{t}$ \\
\hline EOP & 2 & 60 & 7 & 26.1 & 3.34 & 25.3 & 2.33 & 28.1 & 0.427 & 30.5 & 5.64 & 3.65 & 0.700 & 0.699 & 0.898 & & & 0.158 & 0.151 \\
\hline EOP & 2 & 70 & 5 & 25.3 & 3.24 & 26.4 & 2.39 & 30.2 & & & & & 0.667 & 0.737 & 0.894 & 0.942 & 0.580 & 0.153 & 0.146 \\
\hline EOP & 2 & 80 & 1 & 24.8 & 2.90 & 36.4 & 2.34 & 31.4 & 4.28 & 34.5 & 5.67 & 3.58 & 0.648 & 0.805 & 0.854 & & & 0.164 & 0.157 \\
\hline EOP & 2 & 90 & 6 & 25.1 & 2.81 & 38.8 & 2.13 & 26.5 & & & & & 0.736 & 0.755 & 0.844 & 0.941 & 0.603 & 0.174 & 0.165 \\
\hline EOP & 2 & 100 & 3 & 24.7 & 2.88 & 43.5 & 2.46 & 32.1 & 12.3 & 40.8 & 5.34 & 3.39 & 0.635 & 0.855 & 0.825 & 0.943 & 0.591 & 0.166 & 0.158 \\
\hline EOP & 2 & 120 & 8 & 20.5 & 2.92 & 50.3 & 2.51 & 34.9 & 19.8 & 56.4 & 6.91 & 3.03 & 0.601 & 0.862 & 0.798 & 0.949 & 0.591 & 0.112 & 0.107 \\
\hline EOP & 2 & 140 & 9 & 21.4 & 2.80 & 43.6 & & & & & & & & & & & & & \\
\hline EOP & 5 & 40 & 6 & 33.1 & 4.18 & 26.1 & 2.42 & 27.3 & & & & & 0.718 & 0.580 & 0.895 & 0.927 & 0.624 & 0.204 & 0.182 \\
\hline EOP & 5 & 50 & 2 & 28.9 & 3.43 & 22.4 & 2.20 & 24.9 & 0.528 & 22.1 & 4.21 & 3.78 & 0.752 & 0.643 & 0.910 & 0.962 & 0.630 & 0.190 & 0.169 \\
\hline EOP & 5 & 60 & 11 & 27.9 & 2.88 & 32.5 & 2.44 & 27.2 & 0.840 & 25.8 & 4.66 & 3.85 & 0.714 & 0.846 & 0.869 & 0.943 & 0.593 & 0.210 & 0.187 \\
\hline EOP & 5 & 70 & 7 & 27.4 & 3.31 & 20.1 & 2.49 & 27.7 & & & & & 0.708 & 0.750 & 0.919 & 0.918 & 0.557 & 0.177 & 0.157 \\
\hline EOP & 5 & 80 & 13 & 25.0 & 2.82 & 34.4 & 2.27 & 29.3 & 0.864 & 23.2 & 4.18 & 3.24 & 0.681 & 0.804 & 0.862 & 0.936 & 0.621 & 0.172 & 0.153 \\
\hline EOP & 5 & 90 & 1 & 25.7 & 2.79 & 32.4 & 1.93 & 27.0 & & & & & 0.725 & 0.692 & 0.870 & 0.932 & 0.616 & 0.185 & 0.165 \\
\hline EOP & 5 & 100 & 5 & 24.7 & 2.85 & 35.7 & 1.98 & 28.3 & 13.2 & 42.6 & 5.51 & 3.23 & 0.699 & 0.695 & 0.857 & 0.957 & 0.634 & 0.167 & 0.149 \\
\hline EOP & 5 & 120 & 8 & 22.7 & 2.80 & 37.7 & 2.65 & 32.7 & 20.6 & 57.5 & 7.00 & 2.82 & 0.634 & 0.948 & 0.849 & 0.962 & 0.639 & 0.144 & 0.128 \\
\hline EOP & 5 & 140 & 3 & 21.1 & 2.79 & 42.6 & & & & & & & & & & & & & \\
\hline EOP & 5 & 160 & 9 & 20.8 & 2.82 & 40.6 & & & & & & & & & & & & & \\
\hline EOP & 5 & 180 & 12 & 21.8 & 2.81 & 40.4 & & & & & & & & & & & & & \\
\hline EOP & 5 & 200 & 4 & 21.5 & 2.84 & 46.7 & 2.47 & 33.5 & 27.6 & 68.2 & 7.73 & 2.60 & 0.621 & 0.867 & 0.812 & 0.953 & 0.617 & 0.126 & 0.112 \\
\hline EOP & 5 & 250 & 10 & 21.6 & 2.81 & 39.7 & & & & & & & & & & & & & \\
\hline EOP & 10 & 40 & 9 & 33.6 & 4.27 & 24.1 & 2.33 & 25.9 & & & & & 0.744 & 0.546 & 0.903 & 0.959 & 0.659 & 0.206 & 0.165 \\
\hline EOP & 10 & 50 & 2 & 29.9 & 3.53 & 20.6 & 2.44 & 27.0 & 0.513 & 18.6 & 3.54 & 4.10 & 0.714 & 0.692 & 0.917 & 0.948 & 0.629 & 0.197 & 0.158 \\
\hline EOP & 10 & 60 & 8 & 29.3 & 3.39 & 19.6 & 2.41 & 25.4 & 0.0352 & 21.0 & 3.91 & 3.96 & 0.745 & 0.710 & 0.921 & 0.906 & 0.585 & 0.197 & 0.158 \\
\hline EOP & 10 & 70 & 12 & 28.9 & 3.37 & 19.1 & 2.50 & 26.2 & & & & & 0.734 & 0.740 & 0.923 & 0.931 & 0.590 & 0.193 & 0.155 \\
\hline EOP & 10 & 80 & 5 & 27.8 & 2.98 & 29.2 & 2.64 & 29.6 & 0.828 & 21.5 & 3.86 & 3.49 & 0.677 & 0.884 & 0.883 & 0.926 & 0.604 & 0.202 & 0.162 \\
\hline EOP & 10 & 90 & 13 & 27.8 & 2.91 & 25.4 & 2.61 & 29.2 & & & & & 0.689 & 0.899 & 0.898 & 0.941 & 0.630 & 0.207 & 0.166 \\
\hline EOP & 10 & 100 & 1 & 26.6 & 2.91 & 31.2 & 2.39 & 29.1 & 9.52 & 31.6 & 4.13 & 2.98 & 0.691 & 0.820 & 0.874 & 0.936 & 0.623 & 0.189 & 0.152 \\
\hline EOP & 10 & 120 & 10 & 24.0 & 2.79 & 27.8 & 2.52 & 30.6 & 15.4 & 46.0 & 5.75 & 2.92 & 0.670 & 0.904 & 0.888 & 0.945 & 0.637 & 0.161 & 0.129 \\
\hline EOP & 10 & 140 & 11 & 22.9 & 2.78 & 34.9 & & & & & & & & & & & & & \\
\hline EOP & 10 & 160 & 3 & 21.0 & 2.76 & 28.3 & & & & & & & & & & & & & \\
\hline EOP & 10 & 180 & 7 & 22.5 & 2.77 & 32.3 & & & & & & & & & & & & & \\
\hline EOP & 10 & 200 & 4 & 22.5 & 2.78 & 34.3 & 2.68 & 33.1 & 18.8 & 54.7 & 6.81 & 2.71 & 0.626 & 0.965 & 0.862 & 0.962 & 0.635 & 0.141 & 0.114 \\
\hline EOP & 10 & 250 & 6 & 23.1 & 2.77 & 32.4 & & & & & & & & & & & & & \\
\hline
\end{tabular}




\section{Appendix F}

\section{Permission to Use Material}

The letter below grants the author permission to publish the photograph of the Busek BHT-200 included in this document as Figure 1.2. 


\section{BUSEK}

Busek Co. Inc.

11 Tech Circle

Natick, MA 01760-1023

April 27, 2009

Jason Sommerville

815 R. L. Smith Bldg.

1400 Townsend Dr.

Houghton, MI 49931

Dear Mr. Sommerville,

This letter will confirm our recent electronic mail correspondence. You are hereby granted permission to use the photographs of the BHT-200 and BHC-1500 cathode that were forwarded to your attention electronically on April 1, 2009 in your dissertation at Michigan Technological University entitled "HallEffect Thruster-Cathode Coupling: The Effect of Cathode Position and Magnetic Field Topology".

The granted permission extends to any future revisions and editions of the dissertation, including non-exclusive world rights in all languages, and to the prospective publication of the dissertation by UMI. These rights will in no way restrict republication of the material in any other form by Busek Co., Inc. or by those authorized by us. I also confirm that Busek Co. Inc. owns the copyright to the above-described material.

PERMISSION GRANTED FOR THE

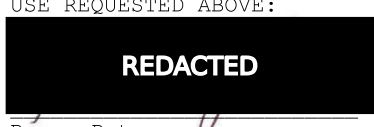

Bruce Pote 7

Director, Hall Thruster Group Busek Co. Inc.
Space Propulsion and Materials Science

Telephone (508) 655-5565

Fax (508) 655-2827

e-mail_busek@busek.com 